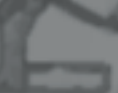

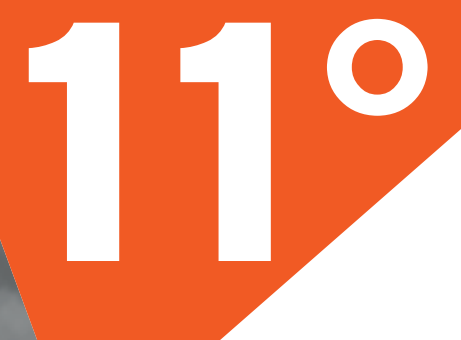
Simposio CEA de Bioingeniería

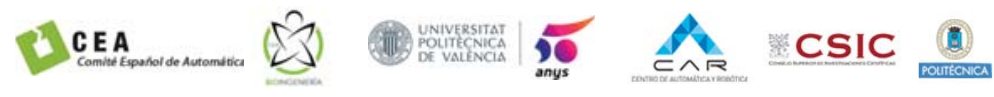
CYTED RESjiste 


\section{Colección Congresos UPV \\ $11^{\circ}$ Simposio CEA de Bioingeniería}

Los contenidos de esta publicación han sido evaluados por el Comité Científico que en ella se relaciona y según el procedimiento que se recoge en

http://ocs.editorial.upv.es/index.php/CEABiolng/CEABiolng/about/editorialPolicies

\section{Editor científico}

Dr. Juan Manuel Belda Lois (Universitat Politècnica de València)

\section{Editado por}

Editorial Universitat Politècnica de València, 2019

www.lalibreria.upv.es / Ref.: 6528_01_01_01

ISBN: 978-84-9048-793-8 (versión impresa)

DOI: http://dx.doi.org/10.4995/CEABiolng.2019.10558

\section{(C) $(1) \Theta$}

$11^{\circ}$ Simposio CEA de Bioingeniería

se distribuye bajo una licencia Creative Commons Reconocimiento-NoComercial-SinObraDerivada 4.0 Internacional. Basada en una obra en http://ocs.editorial.upv.es/index.php/CEABiolng/CEABiolng 


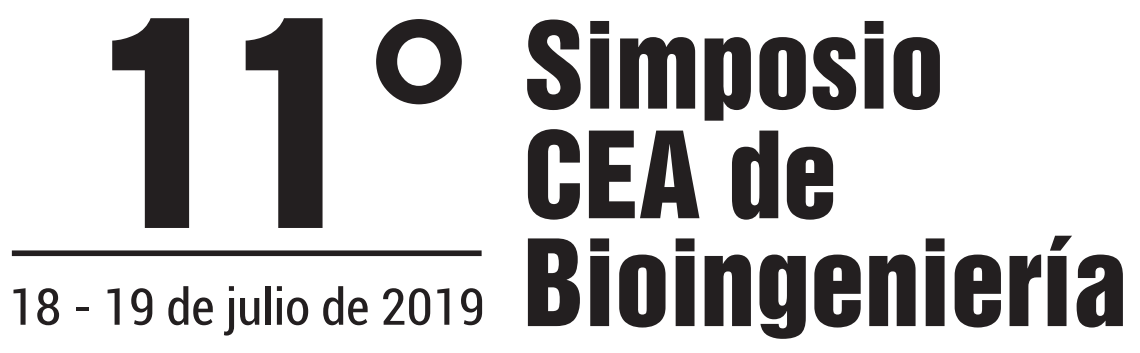




\section{PRESENTACIÓN DEL SIMPOSIO}

En nombre del Comité Organizador, es un privilegio darles la bienvenida al $11^{\circ}$ Simposio CEA Bioingeniería 2019 que se celebra del 18 al 19 de julio en la Ciudad Politécnica de la Innovación de la Universitat Politècnica de València.

El propósito de este simposio, que cumple ahora su undécima edición, es el de proporcionar un espacio de encuentro entre investigadores, desarrolladores, personal clínico, alumnos, industriales, profesionales en general e incluso usuarios que realicen su actividad en el ámbito de la Bioingeniería, principalmente en España e Iberoamérica. Se trata de presentar, compartir y discutir nuevos avances en este campo con aportaciones tanto teóricas como experimentales y de validación e introducción en el mercado de soluciones que traten de cubrir en lo posible las necesidades especiales de personas con deficiencias físicas, sensoriales o cognitivas para ampliar así sus capacidades funcionales de relación, movilidad manipulación y acceso al conocimiento.

En esta edición del Simposio se han presentado trabajos en las áreas de Biomecánica, Interfaces Cerebro-Máquina, Tecnologías para la Rehabilitación y la Asistencia, Uso de Exoesqueletos en el ámbito laboral, Robótica e Imagen Médica y Transferencia de Bioingeniería a la Industria y el Mercado. Los trabajos se han organizado en tres sesiones temáticas moderadas por tres reputados miembros del Comité Científico del Simposio: Dr. José María Azorín Poveda, Dr. Eduardo Rocón de Lima y Dr. Juan Manuel Belda Lois.

Tenemos, además, el honor de contar con la participación de dos ponentes de reconocido prestigio: el Dr. Juan Álvaro Gallego Abella, investigador del Grupo Robotics \& Cybernetics del Centre for Automation and Robotics (CAR-CSIC) de la Universidad Politécnica de Madrid, que impartirá una conferencia plenaria sobre interfaces intracorticales para entender y restaurar el movimiento y D. Israel Benavides, ingeniero Ergónomo de Programas de FORD Automotive que en su conferencia plenaria nos explicará el uso de exoesqueletos en las líneas de producción.

Deseamos que este Simposio CEA de Bioingeniería sea fructífero para todos los participantes y contribuya a su esfuerzo e ilusión por el avance de la Bioingeniería.

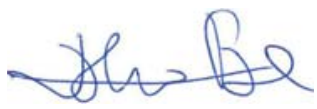

Álvaro F. Page del Pozo

Presidente del Comité Organizador del 11오 Simposio CEA de Bioingeniería 


\section{Simposio CEA de \\ Bioingeniería}

\section{COMITÉ ORGANIZADOR}

Dr. Álvaro Felipe Page del Pozo

Universitat Politècnica de València
Dra. María Amparo Guerrero Alonso

Universitat Politècnica de València

\section{COMITÉ CIENTÍFICO}

Dr. José María Azorín Poveda

Universidad Miguel Hernández de Elche

Dr. Juan Manuel Belda Lois

Universitat Politècnica de València

Dr. Ángel Gil Agudo

Hospital Nacional de Parapléjicos de Toledo

Dr. Miguel A. Mañanas Villanueva

Universidad Politécnica de Cataluña
Dr. Rafael Raya López

Universidad San Pablo CEU

Dr. Eduardo Rocon De Lima

Grupo de Ingeniería Neural y Cognitiva, CAR-CSIC

Dra. Pilar Serra Añó

Universitat Politècnica de València

Dr. Joan Vidal Samsó

Instituto Guttman 


\section{$11^{\circ}$ \\ Simposio \\ CEA de \\ Bioingeniería}

\section{ORGANIZADORES}

CEA

Comité Español de Automática

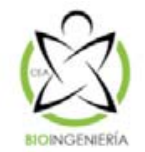

UNIVERSITAT

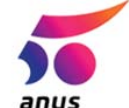

anys
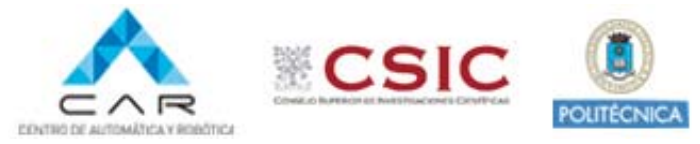

\section{COLABORADORES}

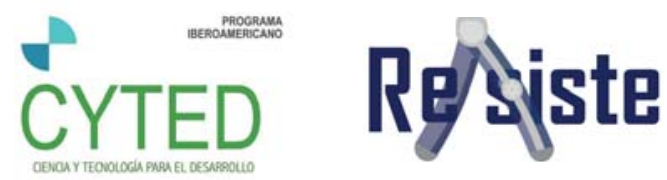




\section{Simposio \\ CEA de \\ Bioingeniería}

\section{ÍNDICE DE SESIONES CIENTÍFICAS}

Jueves, 18 de julio

\section{SESIÓN A. ÁREA: BIOMECÁNICA.}

Patrones de desplazamiento del eje instantáneo de rotación del movimiento de elevación humeral.

Comparación de la normalización lineal de la escala de tiempos con el registro funcional continuo en movimientos cíclicos del cuello.

Validación de los marcadores arUco para el análisis de movimientos humanos.

Fundamentos biomecánicos en el diseño de inmovilizador para rehabilitación de fracturas múltiples.

Análisis biomecánico para confirmar el diagnóstico en neurorrehabilitación.

Cambios en la cinemática articular tras entrenamiento de la marcha con exoesqueleto robótico ambulatorio.

SESIÓN A. ÁREA: ROBÓTICA E IMAGEN MÉDICA.

Sistema no invasivo para la medida y visualización de desplazamientos de tejidos en neurocirugía 


\section{Simposio \\ CEA de \\ Bioingeniería}

SESIÓN B. ÁREA: INTERFACES CEREBRO-MÁQUINA.

Control asíncrono de sistemas $\mathrm{BCl}$ basados en ERP mediante la detección de potenciales evocados visuales de estado estable provocados por los estímulos periféricos del paradigma oddball.

Estudio preliminar de la detección de cambios de velocidad de la marcha a partir de señales EEG.

Posible efecto de interacción entre el paradigma de presentación y el conjunto de estímulos en un teclado virtual controlado a través de una interfaz cerebro-ordenador basada en P300.

Estudio preliminar de la usabilidad de tres tamaños de teclados basados en interfaces cerebro-computador (BCl) por pacientes con ELA.

SESIÓN B. ÁREA: TECNOLOGÍAS PARA LA REHABILITACIÓN Y LA ASISTENCIA.

Evaluación de la interacción entre atención visual y memoria de trabajo: un estudio basado en técnicas de eye-tracking.

Consecuencias de la neuromodulación endógena en el fenómeno de la desincronización de los ritmos sensorimotores en la enfermedad de Parkinson.

Estrategia para el control háptico, basado en electromiografía, de un exoesqueleto de mano para neurorehabilitación.

La estimulación mecánica aferente no reduce sistemáticamente el temblor de pacientes con temblor esencial. 
SESIÓN C. ÁREA: USO DE EXOESQUELETOS EN EL ÁMBITO LABORAL

Estimación de los esfuerzos generados en el hombro por parte de trabajadores de la industria del automóvil mediante modelos biomecánicos de medidas in situ. 080

Desarrollo de un modelo de exoesqueleto de hombro mediante OpenSim ${ }^{\circledR}$.

SESIÓN C. ÁREA: TRANSFERENCIA A LA INDUSTRIA Y EL MERCADO.

Fiabilidad y precisión en Biomecánica. 


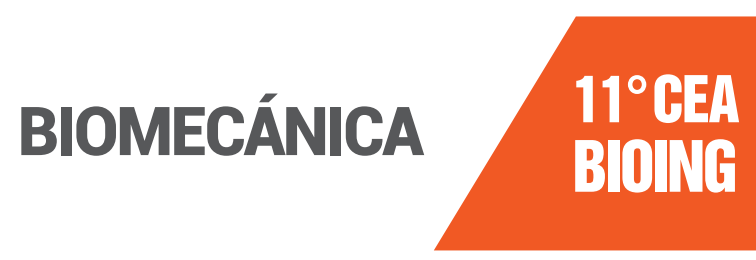




\title{
Patrones de desplazamiento del eje instantáneo de rotación del movimiento de elevación humeral
}

\section{Cáceres, Magda; Page, Álvaro}

Instituto Universitario Mixto de Biomecánica. Universitat Politècnica de València, Valencia, España, magcacerescaceres@gmail.com, afpage@ibv.upv.es.

\begin{abstract}
We present an experimental study for the description of the paths described by the thoracohumeral instantaneous helical axis (IHA) in cyclical movements. The movement was recorded by means of a motion analysis technique with videophotogrammetry that allows the measurement of the IHA with an error lower than $1 \mathrm{~cm}$, in a very reproducible way. This makes it possible to represent the IHA as an axoid, whose mediolateral displacement is related to the angular velocities of the sternoclavicular, acromioclavicular and glenohumeral joints, and whose vertical position is associated with glenohumeral joint elevation. The study was carried out with 41 healthy subjects and 15 subjects with pathologies of the shoulder, analyzing the cyclic movement of elevation-lower of the humerus in the frontal and scapular planes. The location and shape of the axoids is sensitive to the effects of the load and the functional ability of the subjects. The results obtained show that the IHA can be useful in the biomechanical functional assessment of the shoulder, by offering information on the coordination of the different joints that constitute it.
\end{abstract}

Keywords: Biomechanics, joint kinematics, shoulder, instantaneous helical axis, functional assessment.

\section{Resumen}

Se presenta un estudio experimental para la descripción de las trayectorias descritas por el eje instantáneo de rotación (EIR) toracohumeral para movimientos cíclicos de elevación-descenso. Se registra el movimiento mediante una técnica de análisis de movimientos con videofotogrametría que permite la medida del EIR del movimiento relativo húmero tórax con un error inferior a $1 \mathrm{~cm}$ y de forma muy reproducible. Esto permite representar el EIR como un axoide, cuyo desplazamiento mediolateral está relacionado con las velocidades de rotación de las articulaciones esternoclavicular, acromioclavicular y glenohumeral, y cuya posición vertical se asocia a la elevación de la articulación glenohumeral. Se ha realizado un estudio con 41 
sujetos sanos y 15 sujetos con patologías del hombro, analizando el movimiento cíclico de elevación-descenso del húmero en los planos frontal y escapular. La ubicación y forma de los axoides es sensible a los efectos de la carga y del estado funcional del sujeto. Los resultados obtenidos muestran que el EIR puede ser de utilidad en la valoración funcional biomecánica del hombro, al ofrecer información sobre la coordinación de las diferentes articulaciones que lo constituyen

Palabras clave: Biomecánica, cinemática articular, hombro, eje instantáneo de rotación, evaluación funcional..

\section{Introducción}

El estudio de la cinemática del hombro es de gran interés para analizar el efecto de diferentes patologías y establecer criterios objetivos de valoración funcional (López-Pascual et al., 2017). El hombro es una articulación compuesta por múltiples estructuras, que deben actuar en armonía para lograr la realización de movimientos de gran amplitud. Dada su complejidad, el estudio cinemático se suele reducir a la medida de ángulos que evalúan la función escapular (McClure et al., 2006) , clavicular (Ludewig et al., 2009), glenohumeral (Hill et al., 2008) o al ritmo escapulo-humeral (Braman et al, 2009). También se han realizado esfuerzos orientados a desarrollar modelos mecánicos más complejos, revisados por Yang (2010). Sin embargo, la utilidad de tales modelos en la práctica clínica es limitada, dados los problemas que presentan. Así, la medida del movimiento de la escápula o de la clavícula mediante medios no invasivos está afectada por importantes artefactos que limitan la validez de las medidas realizadas (McClure et al, 2001). Además, los modelos mecánicos implican considerar parámetros y características individuales difíciles de determinar, lo que condiciona la validez de dichos modelos (Hatze, 2000). Por el contrario, modelos simples, como los basados en la medida del movimiento húmero-torácico permite un estudio robusto del movimiento conjunto, que ofrece información sobre las alteraciones de los elementos de la cadena cinemática (López-Pascual et al., 2017).

Sin embargo, la mayoría de estudios publicados, revisados en López-Pascual (2015) y Cáceres (2019), se limitan al estudio de variables angulares, lo que supone una simplificación de la cinemática y presenta algunas limitaciones metodológicas. En efecto, la descripción de giros en tres dimensiones es un problema matemático complejo, cuyos resultados dependen del tipo de representación utilizada (López-Pascual et al, 2016). Por otra parte, la composición de giros es un problema no lineal por lo que la relación entre el giro humerotorácico y los giros de la clavícula o escápula no es directa ni evidente (De Rosario et al., 2012). Finalmente, al usar exclusivamente ángulos se está asumiendo implícitamente una 
articulación con tres grados de libertad, ofreciendo una información muy incompleta de la cinemática del complejo articular del hombro.

Una solución de compromiso entre la complejidad de los modelos cinemáticos que consideran los diferentes elementos del complejo articular, y los modelos simplificados que describen la cinemática mediante ángulos, es la representación del Eje Instantáneo de Rotación (EIR). El EIR ha sido utilizado previamente para representar articulaciones complejas como el cuello (Woltring, 1994). Su posición y orientación depende de las velocidades angulares de cada una de las articulaciones que forman un complejo articular, por lo que se ha propuesto como una variable sensible a cambios en la coordinación motora (Page et al, 2011). Sin embargo, su medida experimental es compleja, debido a su sensibilidad a los errores experimentales y a la variabilidad humana (Page et al, 2006). Por ello, son escasos los trabajos en los que se realiza una medida precisa del EIR.

En este trabajo, se presenta un estudio experimental para la descripción de las trayectorias descritas por el eje instantáneo de rotación (EIR) toracohumeral para movimientos cíclicos de elevación-descenso. Se registra el movimiento mediante una técnica de análisis de movimientos con videofotogrametría que permite la medida del EIR del movimiento relativo húmero tórax de forma precisa y reproducible. Esto permite representar el EIR como un axoide, cuyo desplazamiento medio-lateral está relacionado con las velocidades de rotación de las articulaciones esternoclavicular, acromioclavicular y glenohumeral, y cuya posición vertical se asocia a la elevaciónde la clavícula. El método se ha aplicado a una muestra de sujetos sanos y con diferentes patologías del hombro, con la finalidad de mostrar la utilidad del EIR en la identificación de alteraciones funcionales que afecten a la coordinación motora del complejo del hombro.

\section{Material y métodos.}

\subsection{Diseño experimental}

En el estudio participaron dos grupos de voluntarios, uno de personas sanas (41 sujetos, 25 hombres, 16 mujeres), para definir patrones de normalidad, y otro de sujetos con alguna patología de hombro (11 hombres, 4 mujeres), con el objetivo de analizar si el sistema que se propone en este trabajo es capaz de detectar patrones anormales. Los sujetos que participaron en el experimento lo hicieron de forma voluntaria, para lo cual firmaron un consentimiento informado como documento de conformidad con la realización de las prueba. El estudio fue aprobado por el Comité de Ética de la Universitat Politècnica de València. En Cáceres (2019) se describen con detalle los criterios de inclusión y exclusión de cada grupo, así como los criterios para establecer el tamaño mínimo de la muestra. 
Los sujetos realizaron varias sesiones de medida para analizar el movimiento de elevación del brazo en los planos escapular y frontal. Para ello se sentaban en una silla especialmente diseñada para este fin, con un respaldo regulable en altura y un conjunto de cinchas para fijar la posición de la pelvis y tronco y hombro contralateral, dejando libre el movimiento del miembro objeto de estudio (Fig. 1). Se midieron ambos brazos en los dos grupos. El movimiento en cada plano (escapular o frontal) se guió mediante un panel que servía de referencia al sujeto). También se colocó un tope en una posición inicial para uniformizar el movimiento de partida. En cada sesión se repitieron las medidas con dos condiciones de carga: con carga (levantando un mazo de $1 \mathrm{~kg}$ de masa) y sin carga.

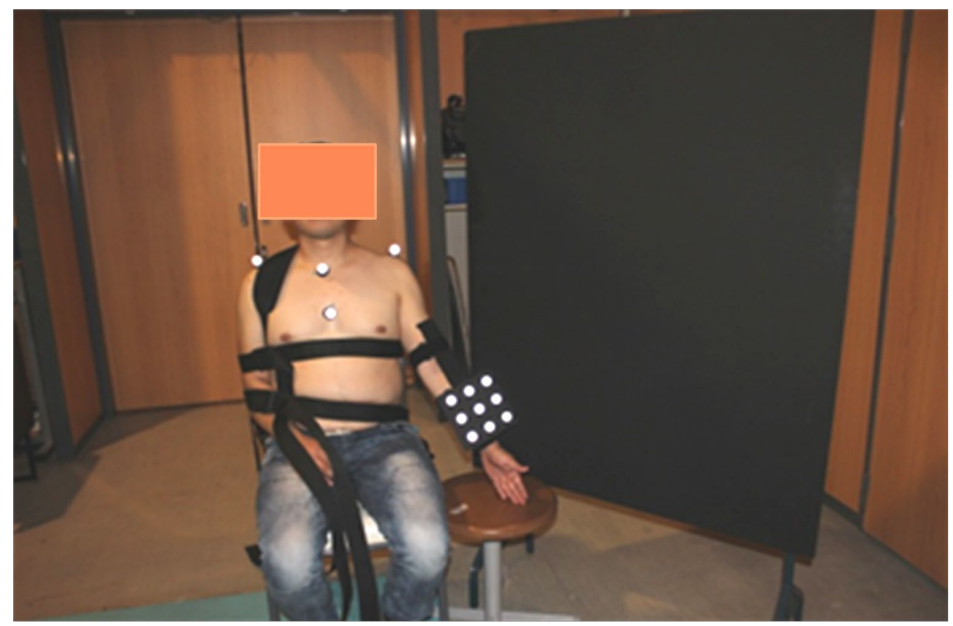

Fig.1. Sistema de medida.

El movimiento del brazo se registró mediante un conjunto de marcadores fijado en la parte anterior del brazo. Además de los marcadores técnicos del brazo, se colocaron marcadores anatómicos en los dos acromion y en el esternón, para la definición del sistema de referencia anatómico y para controlar pequeños movimientos del tronco.

En cada sesión de medida el sujeto debía realizar varios ciclos seguidos de elevación y descenso, desde la posición de referencia hasta la máxima elevación posible. Los movimientos se realizaron a la velocidad deseada por el sujeto.

El movimiento se registró mediante un sistema de videofotogrametría (Kinescan-IBV), a 200 fotogramas por segundo. El sistema proporciona la posición de los marcadores con un error inferior a $0.5 \mathrm{~mm}$, lo que permite medir la posición del EIR con una precisión superior a 0.5 cm, y su orientación con errores inferiores a $1^{\circ}$ (Page et al, 2006, Cáceres et al, 2016)

En Cáceres (2019) se describe con detalle el procedimiento de medida seguido. Además de las medidas cinemáticas, se pasó a los participantes un cuestionario que incluía la Escala Visual Analógica (EVA), para evaluar el nivel de dolor de los sujetos con patología. 


\subsection{Modelo cinemático}

A partir de las coordenadas de los marcadores técnicos y anatómicos proporcionadas por el sistema de videofotogrametría, se realizaron los cálculos cinemáticos usando el modelo y procedimientos descritos en Page et al (2009), que utilizan un método vectorial para el cálculo del movimiento de un sólido respecto a una posición de referencia. En esencia, el sistema de cálculo consta de los siguientes bloques:

- Definición del sistema de referencia anatómico ligado al tronco, de acuerdo con las recomendaciones de la Sociedad Internacional de Biomecánica (Wu et al, 2005).

- Análisis de desplazamientos finitos: para calcular el movimiento de segmento respecto a su posición de referencia (desplazamiento de un punto $\mathrm{y}$ tres giros). Los ángulos se han medido como vector de Rodrigues (Page et al, 2009) y luego transformados a la secuencia de Euler YXY.

- Análisis de velocidades: velocidad lineal y angular en cada instante de tiempo. Cálculo de la posición y orientación del eje instantáneo de rotación.

- Identificación de los ciclos de movimiento (elevación y descenso) y segmentación de la señal continua.

- Obtención del ciclo medio: desplazamientos finitos, velocidades y ejes promedio.

Para representar la posición del EIR de ha calculado el punto $\mathrm{H}$, de corte del eje con el plano del movimiento que pasa por el acromion correspondiente en la posición de referencia. En cada sesión de medida, se obtuvo un único axoide correspondiente al movimiento promedio de los ciclos de dicha sesión. Como se ha comentado, dicho axoide está definido por la orientación del EIR y por el punto $\mathbf{H}\left(\mathrm{y}_{\mathrm{H}}, \mathrm{z}_{\mathrm{H}}\right)$, donde $\mathrm{Y}$ representa la dirección vertical y $\mathrm{Z}$ la medial-lateral. Para poder comparar axoides de sujetos o sesiones diferentes, las coordenadas de $\mathrm{H}$ se han representado en función del procentaje del ciclo de movimiento, $\mathrm{H}(\mathrm{p})$, siendo $\mathrm{p}$ una variable que toma el valor 0 para el punto de mínima elevación y 100 para el de máxima elevación.

Para interpretar la relación entre la forma de la curva $\mathbf{H}(\mathrm{p})$ y la coordinación entre las articulaciones del hombro, partiremos de la figura $2 \mathrm{a}$, donde se muestra una medida. Se ha incluido una representación del esqueleto para dar una idea de la escala (la distancia entre los acromion es la misma en gráfico que en el sujeto representado), si bien en este trabajo no se realizaron radiografías

En cada instante, la posición del EIR viene dada por la posición de los ejes de giro de los tres movimientos del complejo del hombro: movimiento clavícula-esternón, escápula-clavícula y húmero-escápula, cuyos ejes deben estar en las correspondientes articulaciones. Según las leyes de composición de movimientos, el EIR del movimiento conjunto debe estar en el 
centro de gravedad de los tres vectores (Figura 2b). El movimiento que más contribuye a la elevación humeral es el de la articulación glenohumeral; por tanto, es previsible que el EIR del movimiento conjunto esté más cerca de dicha articulación. Sin embargo, en la medida en la que aumente la velocidad de giro de la clavícula, el EIR deberá desplazarse hacia el esternón, en una cantidad que depende de la relación entre las velocidades angulares del húmero, de la clavícula y de la escápula.

a)

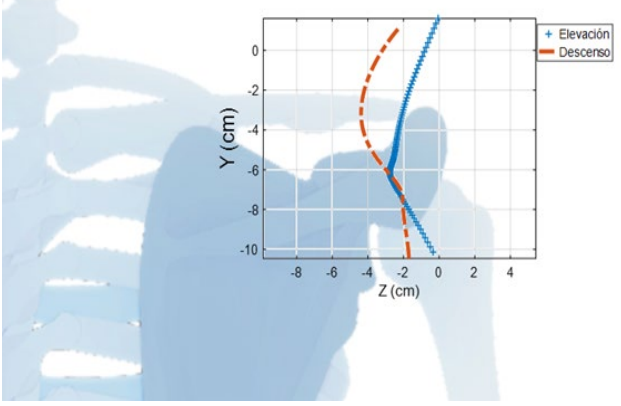

b)

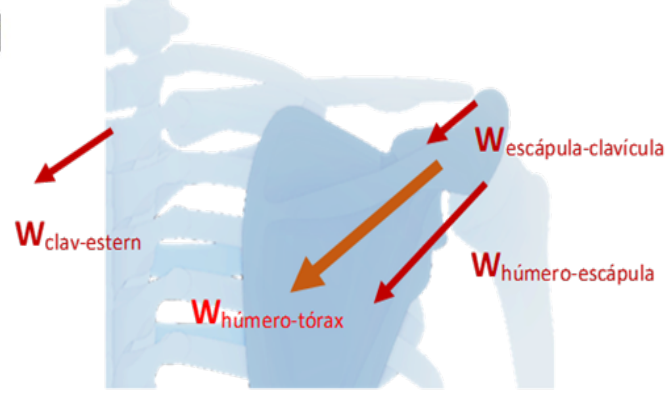

Fig.2. a) Trayectoria descrita por el punto H en un sujeto. b) Composición de movimientos que determina la posición del punto $H$.

En definitiva, la evolución del EIR en la dirección $Z$ es una medida indirecta de la contribución relativa del movimiento de la clavícula a la elevación total. Cuando la contribución de dicho movimiento sea despreciable frente al glenohumeral, entonces el EIR estará cerca de dicha articulación, desplazándose medialmente cuando crezca su aportación relativa. Por otra parte, el desplazamiento vertical está asociado al desplazamiento de las articulaciones clavícula-escápula y glenohumeral, que dependen de la posición de la clavícula.

\subsection{Tratamiento de datos}

Cada registro proporciona una curva $\mathbf{H}(\mathrm{p})$ descrita por la intersección del EIR con el plano del movimiento. La distribución de curvas ha sido analizada usando técnicas de análisis de datos funcionales (FDA) (Ramsay y Silverman, 2005). Se han realizado los siguientes análisis:

1. Análisis descriptivo de la base de normalidad. Se han obtenido las medias y desviaciones típicas funcionales.

2. Análisis del efecto de la carga y el estado funcional sobre las curvas $\mathbf{H}(\mathrm{p})$. Se realizaron ANOVAs funcionales: 
a. En el caso del efecto de la carga, se consideraron los factores lado, carga y plano como factores fijos y el factor sujeto como factor aleatorio.

b. En el estudio del efecto del estado funcional se realizaron dos ANOVAs. En el primero se compararon los EIR de los sujetos sanos con lo patológicos divididos en dos grupos, según el rango de elevación (menos de $135^{\circ}$ o más de $135^{\circ}$ ). En el segundo estudio, sólo se usaron los registros de la base de patología, analizando las diferencias entre trayectorias del EIR a partir de las puntuaciones en la escala EVA de dolor (menos de 4 o igual o mayor a 4)

El análisis de inferencia se ha realizado sobre las curvas correspondientes al movimiento de elevación, ya que el de descenso presentaba una variabilidad muy grande.

\section{Resultados}

La figura 3, muestra las curvas medias de la trayectoria del EIR en la base de sujetos sanos para los movimientos de elevación y descenso. En total, se registraron 135 curvas en la base de sujetos sanos (con diferentes combinaciones de carga y plano de elevación).

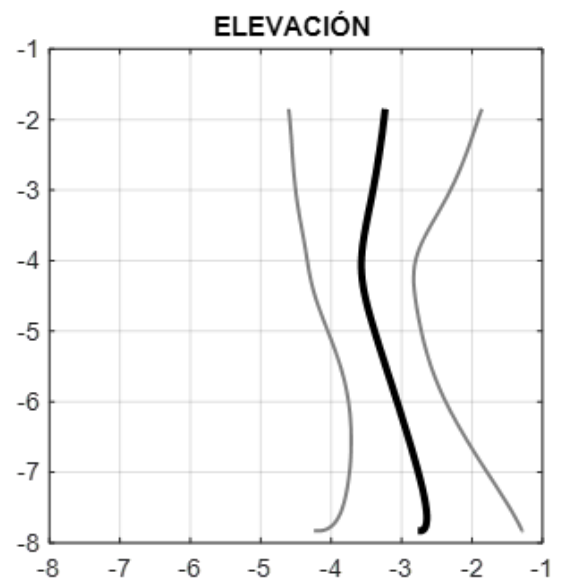

Fig.3 Trayectoria media $Y(Z)$ del EIR en el movimiento de elevación (negro). Unidades en cm, punto de referencia, acromion. El movimiento empieza en la parte inferior.Las curvas laterales en gris corresponden a la media \pm 1 d.t. Representaciónm del brazo derecho, en vista posterior. Los desplazamientos a la derecha son laterales y a la izquierda, mediales.

Como puede observarse, las trayectorias siguen un patrón como el descrito en el modelo cinemático de la Fig.2. El movimiento de elevación arranca unos $8 \mathrm{~cm}$ por debajo del acromion, en una posición correspondiente a la articulación glenohumeral, y se va desplazando hacia arriba, a medida que se eleva dicha articulación por efecto de la elevación 
de la clavícula y la escápula. El rango de desplazamiento vertical del EIR es amplio, del orden de $6 \mathrm{~cm}$ de media. A medida que se eleva, el EIR se desplaza medialmente; según el modelo cinemático, esto se debe a una mayor activación de la articulación esterno-clavicular. A partir de cierto punto (unos $120^{\circ}$ de elevación) el EIR vuelve a desplazarse lateralmente, debido a que disminuye la velocidad de la clavícula respecto a la de la articulación glenohumeral. La dispersión es bastante grande entre sujetos.

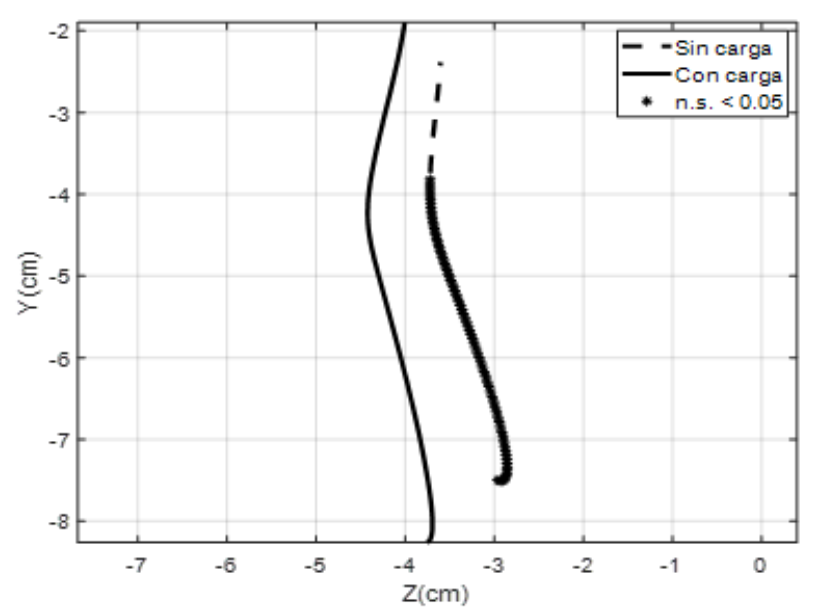

Fig. 4. Medias marginales estimadas de las trayectorias sin carga y con carga. Movimiento de elevación, base de sujetos sanos.

La figura 4, muestra el efecto de la carga. Como puede observarse, el movimiento con carga está desplazado medialmente y tiene un rango de desplazamiento vertical más grande que el movimiento sin carga. Las diferencias son significativas en casi todo el rango del movimiento.

Finalmente, las figuras 5 y 6 representan el efecto del estado funcional, caracterizado por el máximo ángulo de elevación (Fig.5) o la puntuación en la escala EVA de dolor (Fig. 6).

En la figura 5, se comparan las medias marginales de la trayectoria del EIR en el grupo de sanos con dos categorías de patrones patólógicos (105 registros en total): Patológico1, con un nivel de afectación leve o moderado (ángulo máximo de elevación igual o superior a $135^{\circ}$ ) y Patológico2, con afectación más severa (ángulo máximo de elevación inferior a $135^{\circ}$ ). Como puede observarse, las diferencias entre el grupo sano y el Patológico1 son pequeñas. Las trayectorias casi coinciden en la parte central, mientras que el rango de elevación es más grande en el caso de los patológicos. Esto es debido a que arrancan con una mayor velocidad y elevan más la clavícula. En cambio, en el caso del grupo Patológico2 aparecen diferencias claras. La trayectoria del EIR se desplaza medialmente (más aportación relativa del movimiento de la clavícula a la elevación total) y el rango es inferior, ya que el hombro se 
eleva menos. Las diferencias entre el grupo patologico2 y los otros es significativa en casi todo el rango de movimiento.

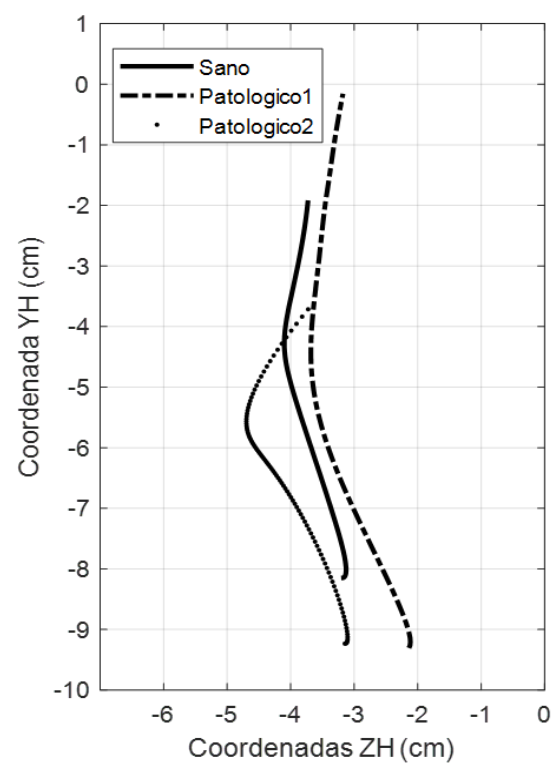

Fig. 5. Medias marginales de las trayectorias del EIR en función del nivel de afectación. Solo movimientos de elevación.

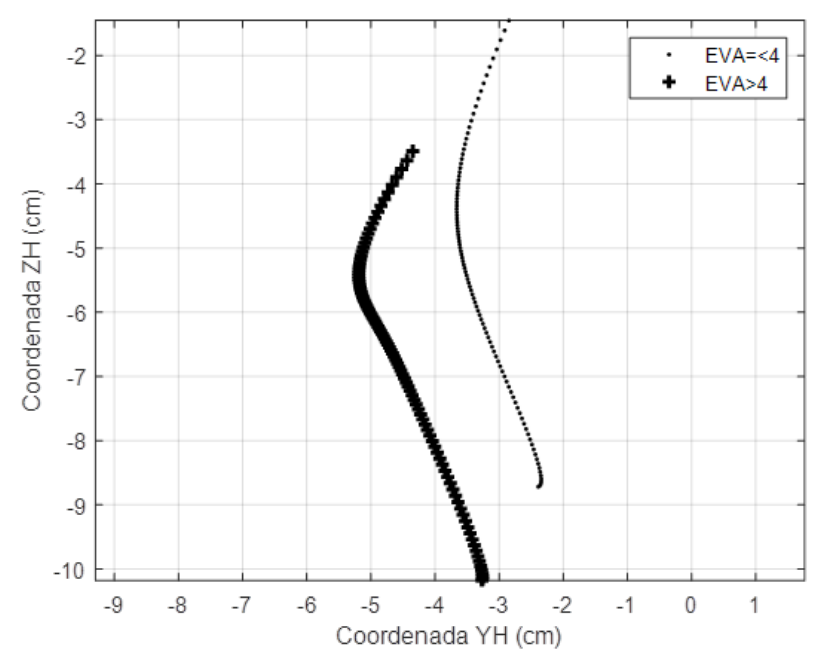

Fig. 6. Efecto de la puntuación EVA en la evolución promedio de la trayectoria de los EIR de la base de sujetos con patología. Medias marginales. Solo movimientos de elevación. 
En la figura 6, se muestran las medias marginales de los registros patológicos, según la puntuación en la escala EVA. El patrón de comportamiento asociado a más dolor es similar al obtenido para menor rango de movimiento. Así, el EIR de los sujetos con más puntuación en la escala está desplazado medialmente y alcanza una altura máxima inferior.

\section{Discusión}

El análisis de la cinemática articular del hombro es uno de los criterios usados en la valoración funcional de esta articulación. Sin embargo, la mayoría de trabajos se basan en el estudio del ritmo escapulo-humeralo en el estudio del movimiento de la escápula, en ambos casos a partir de la medida de ángulos. Como se comenta en la Introducción, este planteamiento presenta, en nuestra opinión, dos problemas. Por una parte, implican la medición del movimiento de la escápula, que está afectado por importantes artefactos (McClure et al., 2001). Por otra, la medida de ángulos es una representación incompleta del movimiento articular, ya que no tiene en cuenta los desplazamientos lineales.

En este trabajo se plantea el uso del EIR como elemento sensible a los cambios en la coordinación entre las tres articulaciones del hombro y se presenta un estudio experimental donde se analizan las trayectorias del EIR y se comparan los movimientos en sujetos sanos y con patología.

La medición de la posición del EIR es difícil, debido a su sensibilidad a los errores experimentales (Page et al., 2006). Sin embargo, con una técnica de medida adecuada es posible medirlo de forma fiable, con errores inferiores a $0.5 \mathrm{~cm}$ (Cáceres, 2016). Por lo que sabemos es el primer trabajo en el que se han medido los axoides del EIR del movimiento toraco-humeral y se han definidos patrones de normalidad y patología.

La trayectoria del EIR permite analizar la contribución en cada instante de las diferentes articulaciones del hombro, en términos de velocidades. Así, el desplazamiento vertical se asocia a una mayor o menor elevación de la clavícula, mientras que el desplazamiento mediolateral depende de las contribuciones de las velocidades angulares en cada articulación al movimiento global. El hecho de trabajar con velocidades y no con ángulos es relevante desde un punto de vista mecánico. En efecto, la descripción de giros depende de la notación utilizada, su composición es compleja, no lineal y difícil de interpretar (De Rosario et al., 2012). Además, los ángulos dependen de la posición de referencia y cambian de forma no lineal cuando se modifica. Por el contrario, las velocidades lineales y angulares, que son las que determinan la posición del EIR, son vectores que representan el estado de movimiento instantáneo. Se componen como una simple suma y su interpretación es bastante sencilla.

Los resultados obtenidos en este trabajo muestran el efecto de la carga sobre la trayectoria del EIR. Su desplazamiento medial se asocia a un mayor contribución de los movimientos de 
la clavícula y del omóplato cuando hay carga. Este hallazgo podría ser explicado por una mayor coactivación muscular del trapecio y el serrato anterior (Ekstrom et al., 2003), e incluso del supraespinoso si se acompaña de rotación externa, en detrimento del deltoides (Reinold et al., 2007).

Las diferencias encontradas entre sujetos sanos y con patología demuestran el potencial del uso del EIR como elemento de valoración funcional. Así, el efecto de una mayor limitación funcional, en términos de menor elevación humeral, va asociado a un desplazamiento medial del eje y a un menor rango de desplazamiento vertical. Estos resultados, pueden ser interpretados por el aumento de la elevación clavicular, producto de una excesiva activación muscular del trapecio superior, descrito en pacientes con patologías de hombro (Cools et al., 2003; Spall et al., 2016). Estas diferencias aparecen también cuando se usa el nivel de dolor como factor.

Hay que señalar que la variabilidad entre sujetos de la trayectoria del EIR es muy grande, especialmente en el grupo de patológicos. Por este motivo, los resultados obtenidos en el trabajo corresponden a diferencias entre patrones promedio. Como en el caso de otras muchas variables biomecánicas, la dispersión entre sujetos hace difícil su uso para identificar patrones sanos o patológicos individuales, especialmente en el caso de sujetos con afectación leve. Sin embargo, es posible que resulten de gran utilidad para evaluar la evolución de un paciente, dado que en este caso dicha variabilidad está controlada. Para comprobar esta hipótesis es necesario realizar estudios longitudinales donde se estudie la evolución en el tiempo.

\section{Conclusiones}

La trayectoria del EIR del hombro está relacionada con la coordinación entre las articulaciones que lo constituyen. Su desplazamiento vertical se asocia a la elevación de la clavícula y el medio lateral a la contribución relativa, en cada instante, de las velocidades angulares de cada articulación.

El efecto de la carga supone una mayor contribución del movimiento clavicular. Este efecto es similar al de una limitación funcional o al dolor, donde también se aprecia el desplazamiento medial del EIR.

Estos resultados muestran el potencial del EIR como descriptor de la coordinación en articulaciones complejas. 


\section{Agradecimientos}

Este trabajo ha sido financiado por el Gobierno de España y cofinanciado por Fondos Feder de la UE gracias al proyecto PDI2017-84201-R (IMBIO3R).

\section{Referencias}

BRAMAN, J. P., ENGEL, S. C., LAPRADE, R. F., \& LUDEWIG, P. M. (2009). « In vivo assessment of scapulohumeral rhythm during unconstrained overhead reaching in asymptomatic subjects ». Journal of Shoulder and Elbow Surgery, vol. 8, issue 6, p. 960-967.

CACERES, M., SERRA, P., LOPEZ, J., PAGE, A. (2016). « Reliability of the Shoulder Instantaneous Helical Axis measurement during the scaption movemen »t. Gait and Posture, vol 49, pp. 268.

CÁCERES, M.L. (2019). Aplicación de la cinemática articular a la valoración funcional del hombro. Tesis Doctoral. Valencia : Universitat Politècnica de València.

COOLS, A. M., WITVROUW, E. E., DECLERCQ, G.A., et al. (2003). « Scapular muscle recruitment patterns: trapezius muscle latency with and without impingement symptoms ». The American Journal of Sports Medicine, 31(4), 542-549)

DE ROSARIO, H., PAGE, A., MATA, V., BESA, A.J., MORENO, R. (2012). « Propagation of artifact errors on kinematic variables. Effect on Euler angles ». Journal of Biomechanics, vol. 45, pp. 293.

HATZE, H. (2002). "The fundamental problem of myoskeletal inverse dynamics and its implications ». Journal of Biomechanics, vol. 35, issue 1, p. 109-115.

LOPEZ PASCUAL, J. (2015). Caracterización cinemática de la elevación humeral en el plano escapular. Definición de patrones de normalidad y análisis del gesto patológico. Tesis Doctoral. Valencia : Universitat Politécnica de Valéncia.

LOPEZ-PASCUAL, J., PAGE, Á., SERRA-AÑO, P. (2017). «Dynamic thoracohumeral kinematics are dependent upon the etiology of the shoulder injury ». PloS one, vol. 12, issue 8, e0183954.

LUDEWIG, P. M., PHADKE, V., BRAMAN, J. P., HASSETT, D. R., CIEMINSKI, C. J., Y LAPRADE, R. F. (2009). « Motion of the shoulder complex during multiplanar humeral elevation ». The Journal of Bone y Joint Surgery, vol. 91, issue 2, p. 378-389.

PAGE, A., DE ROSARIO, H., MATA, V., HOYOS, J. V., PORCAR, R. (2006). Effect of marker cluster design on the accuracy of human movement analysis using stereophotogrammetry. Medical and Biological Engineering and Computing, 44(12), 1113-1119.

PAGE, Á., DE ROSARIO, H., MATA, V., Y ATIENZA, C. (2009). «Experimental analysis of rigid body motion. A vector method to determine finite and infinitesimal displacements from point coordinates ». Journal of Mechanical Design, vol. 131, issue 3, pp. 031005.

PAGE, Á., DE ROSARIO, H., GALVEZ, J. A., Y MATA, V. (2011). « Representation of planar motion of complex joints by means of rolling pairs. Application to neck motion ». Journal of biomechanics, vol. 44, issue 4, pp. 747-750. 
McCLURE, P. W., MICHENER, L. A., Y SENNETT, B. (2001). « Dynamic measurements of threedimensional scapular kinematics: a validation study ». Journal of biomechanical engineering, vil. 123, issue 2, p. 184-190.

McCluRE, P.W., MICHENER, L. A., KARDUNA, A. R. (2006). «Shoulder function and 3dimensional scapular kinematics in people with and without shoulder impingement syndrome». Physical Therapy, vol. 86, issue 8, p. 1075-1090.

RAMSAY, J. O., Y SILVERMAN, B. W. (2005.). Functional data analysis. Springer, New York.

REINOLD, M. M., MACRINA, L. C., WILK, K.E., et al.. (2007). « Electromyographic analysis of the supraspinatus and deltoid muscles during 3 common rehabilitation exercises ». Journal of athletic training, vol. 42, issue 4, pp.464.

SPALL, P., RIBEIRO, D. C., Y SOLE, G. (2016). «Electromyographic activity of shoulder girdle muscles in patients with symptomatic and asymptomatic rotator cuff tears: a systematic review and meta-analysis ». PMyR, vol. 8, issue 9, pp.894-906.

WOLTRING, H. J., LONG, K., OSTERBAUER, P. J., FUHR, A. W. (1994). «Instantaneous helical axis estimation from 3-D video data in neck kinematics for whiplash diagnostics ». Journal of biomechanics, vol. 27, issue 12, pp. 14151427-14251432.

WU, G., VAN DER HELM, F. C., VEEGER, H. E. J., et al. (2005). «ISB recommendation on definitions of joint coordinate systems of various joints for the reporting of human joint motion-Part II: shoulder, elbow, wrist and hand. Journal of biomechanics », vol. 38, issue 5, pp. 981-992.

YANG, J., FENG, X., KIM, J. H., Y RAJULU, S. (2010). « Review of biomechanical models for human shoulder complex ». International Journal of Human Factors Modelling and Simulation, vol. 1, issue 3, p. 271-293. 


\title{
Comparación de la normalización lineal de la escala de tiempos con el registro funcional continuo en movimientos cíclicos del cuello
}

\author{
López-Pérez, Noelia ${ }^{a}$; Venegas, William ${ }^{\text {; }}$ Serra-Añó, Pilarc; Page, Álvaro ${ }^{\text {d }}$ \\ ${ }^{a}$ E.T.S. I. Industriales. Universitat Politècnica de València (España); noelope1@etsii.upv.es. \\ bEscuela Politécnica Nacional de Quito (Ecuador); William.venegas@epn.edu.ec \\ 'Facultat de Fisioterapia, Universitat de València (España); pilar.serra@uv.es \\ dnstituto Universitario Mixto de Biomecánica, Universitat Politècnica de Valencia (España); \\ afpage@ibv.upv.es
}

\begin{abstract}
The time scale normalization is a necessary step in the analysis of human movements. The standard technique is linear normalization that may be ineffective in reducing the variability in the duration of events. An alternative is the registration of functions that involves adjusting the time scale in a nonlinear way, a complex and computationally expensive procedure.

In this work, both methods are compared for the cyclic movement of flexionextension of the neck. From a database with 437 complete cycles, we have analyzed the differences in mean curves and functional standard deviations, whose mean values and confidence intervals have been established through a bootstrapping process.
\end{abstract}

The results show small, although significant, differences in the average curves obtained, with a greater amplitude in the angles and velocities. The functional standard deviations are somewhat lower when registration is used. These results suggest that the continuous registration, although it may be useful for the analysis of non-periodic signals, offers no significant advantages over linear normalization in the case of cyclic movements.

Keywords: Human movement analysis, linear normalization of the time scale, Registration of functions; Functional Data Analysis.

\section{Resumen}

La normalización de la escala de tiempos es un paso necesario en el análisis de movimientos humanos. La técnica estándar es la normalización lineal que puede ser ineficaz para reducir la variabilidad en la duración de los eventos. Una alternativa es el registro continuo que supone ajustar de forma no lineal la escala de tiempo, procedimiento complejo y computacionalmente costoso. 
Comparación de la normalización lineal de la escala de tiempos con el registro funcional continuo en movimientos cíclicos del cuello

En este trabajo se comparan ambos métodos para el movimiento cíclico de flexo-extensión del cuello. A partir de una base de datos con 437 ciclos completos, se han analizado las diferencias en las curvas medias y las desviaciones típicas funcionales, cuyos valores medios e intervalos de confianza se han establecido mediante un proceso de bootstrapping.

Los resultados muestran pequeñas, aunque significativas, diferencias en las curvas medias obtenidas, con una mayor amplitud en los ángulos y velocidades. Las desviaciones típicas funcionales son algo menores en las curvas registradas. Estos resultados sugieren que el registro no lineal, aunque puede ser útil para el análisis de señales no periódicas, no ofrece ventajas importantes frente a la normalización lineal en el caso de los movimientos cíclicos.

Palabras clave: Análisis de movimientos humanos, Normalización lineal de la escala de tiempos, Registro de funciones; Análisis de Datos Funcionales.

\section{Introducción}

Los estudios clásicos sobre Biomecánica describen el movimientos humanos mediante variables numéricas tales como máximos, mínimos, rangos de movimiento, velocidades y aceleraciones máximas, duración de fases del movimiento, etc. (Winter, 1991; Ciriello et al, 1991; Novacheck, 1998). Este planteamiento supone reducir la información contenida en las funciones continuas que representan todas las variables cinemáticas o dinámicas y limitar la posibilidad de analizar las relaciones existentes entre las variables y sus derivadas.

A partir del año 2000 surge un cambio en la Estadística clásica motivado por la publicación de varios trabajos sobre una nueva forma de abordar el estudio de señales continuas, denominada Análisis de Datos Funcionales (DFA) (Ramsay \& Silverman, 2005). El DFA plantea una alternativa en la que se considera el carácter continuo de las variables de movimiento y permite desarrollar técnicas de tratamiento adaptadas a ese tipo de datos. La idea básica del Análisis de Datos Funcionales (FDA) reside en la descripción de cada observación como una función continua dependiente del tiempo (Lucero, 2000; Mechmeche, et al, 2016). Los métodos de FDA son capaces de extraer información, contenida en las señales o funciones y sus derivadas, que no se encuentra normalmente aplicando métodos de la estadística tradicional (Ullah \& Finch, 2013).

Sin embargo, para que las curvas de diferentes pruebas o sujetos sean comparables, es necesario ajustar la base de tiempos de alguna manera, de forma que se separe la variabilidad asociada a la amplitud de la relacionada con diferencias en el patrón temporal 
(Liebl \& Mosler, 2012). En efecto, las diferencias de fase introducen mucha variabilidad en la muestra de curvas, lo que hace muy difícil su comparación directa y determina que los valores medios no representen una buena estimación del conjunto (Sørensen \& Sangalli, 2013; Crane et al., 2011). Es en estos casos en los que se debe recurrir al uso de algún método o técnica para eliminar la variabilidad de fase, manteniendo la información de la función contenida en la forma y la amplitud de las señales.

La técnica estándar usada en biomecánica es la normalización lineal de la escala de tiempos, que consiste en transformar la base de tiempos en una escala de 0 a $100 \%$ del tiempo de ejecución del movimiento. Es una técnica simple y efectiva (Boulgouris et al., 2006). No obstante, otros estudios han demostrado que dicha técnica puede ser inadecuada en determinados casos. En particular, en el trabajo de Page y Epifanio (2007) se definen los criterios matemáticos que determinan la la efectividad de la normalización lineal en la reducción de la variabilidad de los datos, basándose en la correlación entre la duración total de cada ciclo y la duración de los eventos principales de la función.

Por ello se han planteado otras alternativas, como el registro de funciones. Se trata de una técnica de normalización no lineal, que expande o contrae localmente la escala de tiempos para buscar la mejor adaptación entre dos curvas, de manera que se minimice el efecto de las diferencias de fase (Ramsay \& Li, 1998). Los métodos de registro de funciones eliminan prácticamente toda la variabilidad de fase, de manera que las curvas registradas tienen la misma forma, y sólo se diferencian por las distintas amplitudes. Esto permite obtener buenas estimaciones de las curvas promedio. Sin embargo, la información temporal (que en la normalización lineal se mantiene en los desfases entre curvas), no desaparece en este tipo de reescalado, sino que se mantiene en las "time warping functions". Estas funciones representan la relación entre el tiempo modificado en función del tiempo real, $h(t)$ y sirven para analizar en qué momentos el movimiento se desarrolla más deprisa o más lento que el promedio del movimiento (Page et al, 2006; Crane et al., 2010).

A pesar de lo atractivo que resulta el registro no lineal de funciones, hay que señalar que presenta algunas limitaciones. En primer lugar, hay que destacar que su efectividad en la reducción de variabilidad depende del tipo y características de las funciones a registrar. Así, cuando se aplica a curvas de movimiento que presentan un gran número de puntos característicos (máximos, mínimos, puntos de inflexión) la reducción en la variabilidad es mucho mejor que cuando se aplica a curvas con pocos puntos característicos (Crane et al., 2010). Además, el proceso de registro es complejo desde el punto de vista matemático, y muy costoso desde el punto de vista computacional, ya que las curvas deben registrarse una a una respecto a una de referencia (típicamente una media). $\mathrm{Si}$, como es frecuente, se usa un proceso procústeo de adaptación de la media, entonces el cálculo se hace muy pesado (Page et al, 2006). Finalmente, el registro de funciones se basa en criterios meramente geométricos que no tienen en cuenta la relación dinámica entre las funciones y sus 
derivadas. Por ello, es posible que los registros obtenidos con una función o su derivada arrojen resultados distintos, lo que no tiene sentido desde el punto de vista físico

En definitiva, cabe valorar las ventajas comparativas del registro frente a la normalización no lineal y evaluar si la mejora en la obtención de curvas promedio compensa la complejidad o posibles patrones anómalos asociados al registro no lineal.

En esta línea se plantea el presente trabajo, cuyo objetivo es comparar ambos tipos de normalizaciones en una amplia base de curvas de flexión-extensión del cuello, analizando tanto los funciones correspondientes al ángulo como a la velocidad angular. En particular comprobaremos las diferencias a la hora de determinar las medias y desviaciones típicas funcionales y las diferencias obtenidas por el registro según se usen los ángulos o las velocidades angulares.

\section{Material y métodos}

\subsection{Muestra de estudio y funciones analizadas.}

Las funciones utilizadas en este trabajo proceden de un estudio sobre el movimiento del cuello en el que participaron 15 sujetos que fueron medidos en dos sesiones diferentes y por dos operadores distintos. Los sujetos fueron voluntarios sanos que firmaron el correspondiente consentimiento informado, de acuerdo con un protocolo aprobado por el Comité de Ética de la Universitat de València.

En cada sesión de medida el sujeto realizó un movimiento continuo de flexo-extensión, describiendo varios ciclos seguidos del movimiento sin detenerse. El protocolo está descrito en Baydal et al.(2011).

\subsection{Captura de movimientos y procesado de las señales.}

El movimiento fue medido con un equipo de fotogrametría (Kinescan-IBV) a 200 fotogramas por segundo. El sistema proporciona las coordenadas de los marcadores. A partir de dichas coordenadas se utilizó software propio para la medida del ángulo de flexoextensión y su derivada (Baydal. 2011). Posteriormente, el movimiento continuo fue dividido en ciclos, cada uno de los cuales es un observación de la muestra. En total se obtuvieron 437 ciclos completos. 


\subsection{Normalizacion lineal y registos}

Sobre la base de 437 ciclos se efectuaron dos normalizaciones:normalización lineal y registro de funciones.

\subsubsection{Normalización lineal}

Se normalizaron las medidas de velocidades cambiando la base de tiempos por un procentaje de la duración de cada ciclo, de manera que el tiempo en el instante i, de la curva $\mathrm{j}, \mathrm{t}_{\mathrm{ij}}$, se transforma en $\mathrm{p}_{\mathrm{ij}}$ :

$$
p_{i j}=100 \times \frac{t_{i j}}{T_{j}}
$$

Donde $\mathrm{T}_{\mathrm{j}}$ es la duración de la curva $\mathrm{j}$. Las curvas normalizadas se interpolaron para obtener un conjunto de 101 valores, desde $\mathrm{p}=0$ hasta $\mathrm{p}=100$.

\subsubsection{Registro de funciones}

Para la normalización no lineal se usó el procedimiento descrito en Page et al. (2006). Con el registro de funciones se pretende encontrar una transformación de la escala de tiempos, $\mathrm{h}_{\mathrm{i}}$, que permite deformar la curva inicial para minimizar las diferencias de fase:

$$
x_{i}^{*}(t)=x_{i}\left(h_{i}(t)\right)
$$

La función $h_{\mathrm{i}}$ se denomina warping function, y debe ser una función continua, derivable y monótona creciente. Estas funciones representan una transformación no lineal de la escala de tiempos, de manera que el tiempo se contrae o dilata localmente para ajustar la forma de las curvas. Por tanto, contienen la información sobre las diferencias temporales que se pierde cuando se alinean las curvas de forma continua al registrarlas. En definitiva, representan la variabilidad en la fase, mientras que las curvas registradas contienen la variabilidad asociada a las diferencias de amplitud.

El criterio usado para minimizar las diferencias de fase consiste en mimizar la función $\mathrm{F}$ (Page et al., 2006)

$$
F\left(h_{i}\right)=\log \mu_{2}(\Sigma)
$$

Donde $\mu_{2}$ es el menor valor propio de la matriz $\Sigma$, que corresponde con una matriz de varianzas-covarianzas de las curvas que se van a registrar:

$$
\Sigma=\left(\begin{array}{cc}
\int x_{0}^{2}(t) d t & \int x_{0}(t) x_{i}(t) d t \\
\int x_{0}(t) x_{i}(t) d t & \int x_{i}^{2}(t) d t
\end{array}\right)
$$


El proceso se registro se realizó utilizando las curvas de velocidad, ya que se recomienda usar derivadas en vez de las funciones medidas (Ramsay y Silverman, 2005).

\subsubsection{Análisis estadístico}

Para evaluar las diferencias entre los tres conjuntos de funciones (brutas, normalizadas linealmente y registradas) se han realizado los siguientes análisis:

1) Análisis descriptivo, representando las familias de curvas y sus medias. Se ha realizado tanto para las curvas de posición como para las de velocidad.

2) Comparación entre los dos métodos de normalización. Para cada familia de curvas (ángulo y velocidad angular) se han calculado dos variables que cuantifican el nivel de alineamiento entre las curvas tras la normalización:

a. Rango de la curva media. Se asume que las curvas con más diferencia de fase presentan un efecto de compensación en los máximos y mínimos, de manera que la curva media tiene un rango menor.

b. Valor máximo de la desviación típica funcional. Las curvas desfasadas presentan valores de desviación típica muy grandes en los puntos de inflexión. Se asume que el método que mejor ajuste los desfases, tendrá menores valores máximos de la desviación típica

Se ha elaborado un contraste estadístico para saber si las diferencias entre los dos métodos son significativas. Para ello se ha utlizado un proceso de bootstrap (con extracción de 5000 muestras con reemplazo) obteniedo en intervalo de confianza de la diferencia. Ajustando el valor de alfa para que el límie inferior sea muy próximo a cero se obtiene en nivel de significación (Efron, 1994).

\section{Resultados}

La Figura 1 muestra la distribución de la familia de curvas con la escala de tiempos sin normalizar. Como puede observarse, las curvas presentan una gran dispersión en amplitud y en la duración de los ciclos.

Como consecuencia de estas diferencias, los valores medios están algo truncados en los valores extremos. Además la curva media se vuelve inestables y su forma está toalmente alterada al final del movimiento. 

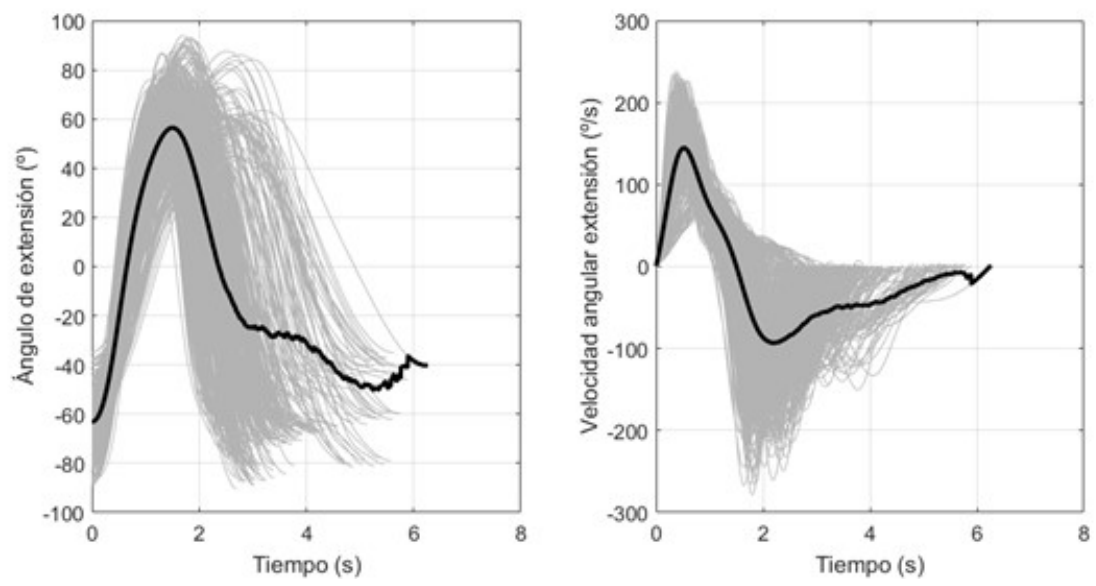

Fig. 1. Ángulo y velocidad angular con la base de tiempos bruta. En gris las 437 curvas usadas. La línea negra representa la media.

Las figuras 2 y 3 muestran la misma representación, pero con la normalización lineal (Fig. 2) y tras el registro de funciones (Fig.3). La normalización lineal proporciona curvas suaves. No obstante, se mantienen ciertas diferencias de fase, lo que se puede traducir en una disminución del valor máximo y mayor dispersión.
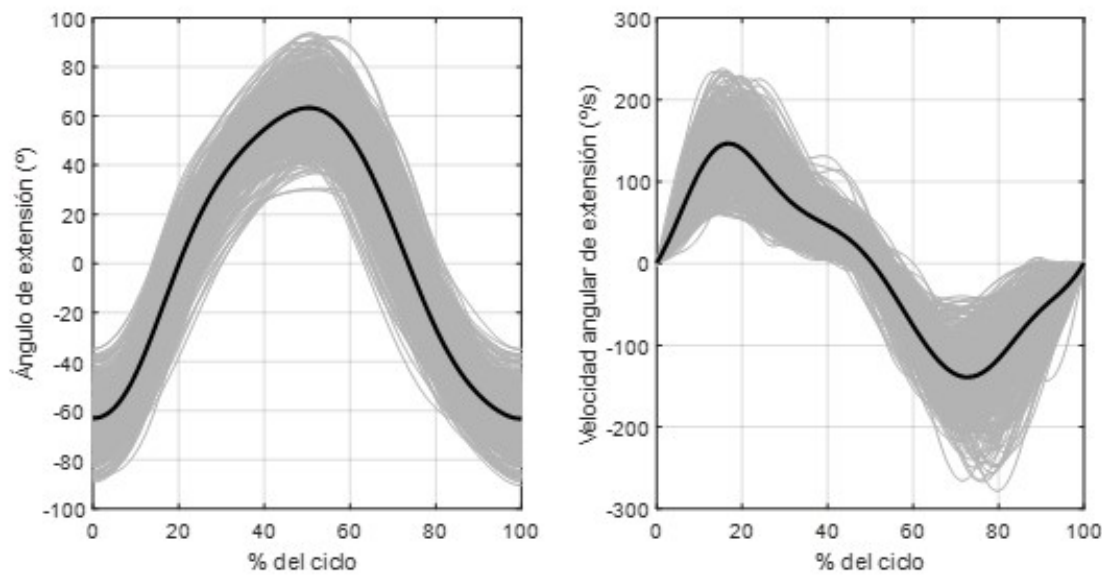

Fig. 2. Angulo y velocidad angular con la base de tiempos normalizada linealmente. En gris las 437 curvas usadas. La línea negra representa la media.

Por el contrario, las curvas registradas presentan un alineamiento mejor, especialmente en las curvas de velocidad, que han sido las usadas para obtener el registro. Sin embargo, en 
las curvas de los ángulos se aprecian ciertas deformaciones en algunas curvas que, una vez registradas no mantienen la forma original. Por este motivo la curva media tienen una pequeña deformación.
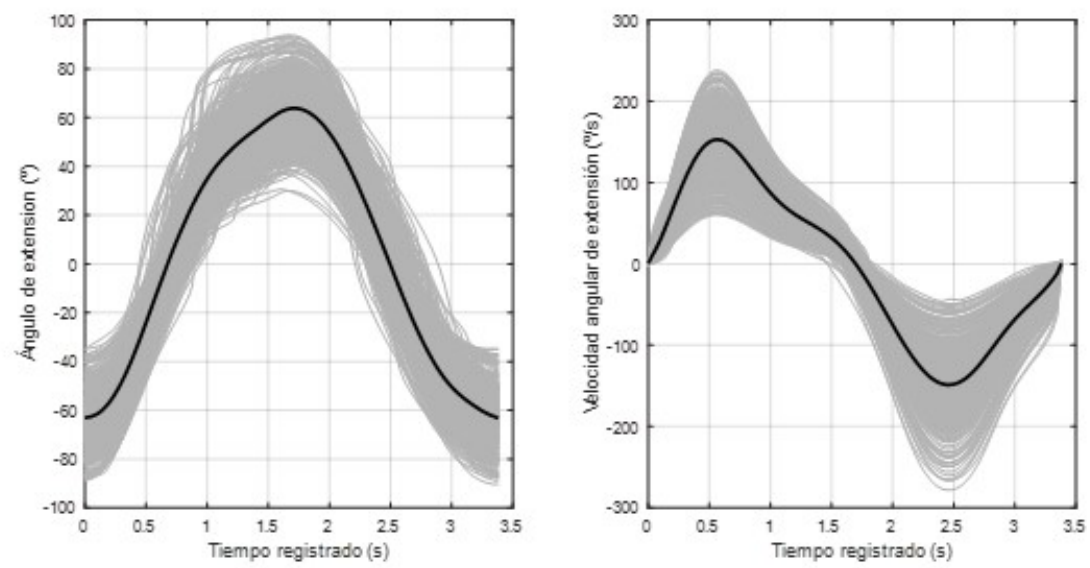

Fig. 3. Ángulo y velocidad angular con la base de tiempos normalizada mediante registro de funciones. En gris las 437 curvas usadas. La linea negra representa la media.

Tabla 1. Diferencias entre la curvas originales, la normalización lineal y la normalización no lineal. Los niveles de significación se refieren a las diferencias entre la normalización no lineal y el registro de funciones.

\begin{tabular}{ccccc} 
& $\begin{array}{c}\text { Sin } \\
\text { normalizar }\end{array}$ & $\begin{array}{c}\text { Normalización } \\
\text { lineal }\end{array}$ & $\begin{array}{c}\text { Registro no } \\
\text { lineal }\end{array}$ & $\begin{array}{c}\text { Nivel } \\
\text { significación }\end{array}$ \\
\hline $\begin{array}{c}\text { Amplitud de la curva } \\
\text { media de angulos }\left(^{(}\right)\end{array}$ & 119.5 & 126.6 & 127.1 & $<0.01$ \\
$\begin{array}{c}\text { Amplitud de la curva } \\
\text { media de velocidades }\left(\mathbf{(}^{\circ} / \mathbf{s}\right)\end{array}$ & 237.8 & 285.5 & 301.6 & $<0.0001$ \\
$\begin{array}{c}\text { Valor máximo, DT } \\
\text { funcional. Ángulo }\left({ }^{(}\right)\end{array}$ & 44.0 & 15.7 & 13.4 & $<0.0001$ \\
$\quad \begin{array}{c}\text { Valor máximo. DT } \\
\text { funcional. Velocidades }(\mathbf{\%} / \mathbf{s})\end{array}$ & 76.2 & 47.2 & 42.7 & $<0.0001$ \\
\hline
\end{tabular}

Las diferencias entre métodos de normalización se resumen en la Tabla 1. Como puede observarse, ambos métodos de normalización mejoran notablemente los parámetros 
obtenidos para las curvas brutas: tienen un rango mucho mayor y hay una disminución drástica de la desviación típica funcional.

En cuanto a las diferencias entre métodos de normalización, el registro no lineal proporciona medias con un rango significativamente mayor al de la normalización lineal , y alinea las curvas de manera que la desviación típica máxima es inferior. No obstante, las diferencias son relativamente pequeñas frente al rango. Por otra parte, también hay una disminución notable en las máximas desviaciones típicas de las curvas brutas y tras la normalización, tanto si se hace linealmente como con registro de funciones. Las diferencias entre métodos son mucho más pequeñas; si bien el registro proporciona curvas con desviaciones típicas significativamente inferiores, la tales diferencias son pequeñas si se comparan con el rango de los ángulos o las velocidades.

\section{Discusión}

La normalización de la escala de tiempos es un paso necesario para comparar las magnitudes asociadas al movimiento humano. Tradicionalmente, el problema de las diferencias en la duración y fases de las curvas asociadas al movimiento se ha resuelto mediante una normalización lineal. Esto puede dar lugar a problemas difíciles de resolver en algunos casos, como se discute en Page et al. (2006) en un estudio sobre fuerzas al sentarse y levantarse. Por este motivo, se han planteado otros procedimientos más complejos para un ajuste no lineal del tiempo, como el método del registro de funciones. Esta técnica se ha usado con éxito en muchas aplicaciones (Page et al, 2006; Sørensen \& Sangalli, 2013).

A pesar de las ventajas que puede puede presentar, esta técnica no está exenta de problemas como los señalados en Crane et al. (2010), quien sugiere que el registro de funciones no difiere de la normalización lineal en movimientos armónicos. Esta hipótesis es compatible con los criterios señalados en Page \& Epifanio (2007): cuando la duración de cada evento es proporcional a la duración del ciclo completo, como sucede en un movimiento armónico, entonces el ajuste lineal es totalmente efectivo.

Sin embargo, no se ha analizado el comportamiento los diferentes métodos de normalización en movimientos cíclicos, pero no armónicos, como es el caso del movimiento cíclico de flexo-extensión del cuello. Este es un movimiento con una armonía relativamente baja (Baydal et al., 2011) ya que no se trata de un movimiento puramente mecánico, como sucede en otros, como la elevación del brazo por ejemplo (Cáceres, 2019).

Los resultados obtenidos muestran que tanto la normalización lineal como el registro de funciones proporcionan una importante reducción de la variabilidad asociada a las 
diferencias de fase. La alineación de las curvas es mejor en el caso del registro, si bien hay algunos matices.

Respecto a las curvas de flexo-extensión, el método del registro produce deformación local en algunas curvas, debidos a cambios acusados en las funciones warping. Sin embargo, la media tiene una amplitud ligeramente superior a la obtenida con la simple normalización lineal. Aunque las diferencias son significativas, son realmente pequeñas si se comparan con el rango (menos del $0.5 \%$ ). Lo mismo sucede con la máxima desviación típica que presenta pequeñas diferencias entre los dos métodos. Teniendo en cuenta que el registro altera de forma anormal la forma de algunas curvas, cabe plantearse si en este caso tiene más ventajas o inconvenientes que el método más simple.

En cuanto a las curvas de velocidad, aquí las diferencias son algo más acusadas. Las curvas de velocidad registradas son suaves y mantienen formas muy similares, sin deformaciones. Proporcionan una media algo mayor que la obtenida tras la normalización lineal y también reduce algo más la variabilidad, como se ve en las desviaciones típicas. Por ello, parece que la normalización no lineal es algo más efectiva que la lineal en las curvas de velocidad

Estas diferencias entre la efectividad en las funciones de posición y de velocidad pueden deberse, en nuestra opinión, a dos factores. Por una parte a que el registro se ha hecho tomando las velocidades como función a registrar, aplicando posteriormente las funciones warping a las posiciones. Por otra, debido a que las curvas de velocidad se parecen menos a una función armónica que las de posición.

En cualquier caso las diferencias son pequeñas y la complejidad matemática y coste computacional del registro de funciones puede no compensar el uso de esta técnica en movimientos cíclicos.

\section{Conclusiones}

A pesar de su sencillez la normalización lineal de la escala de tiempos es una técnica efectiva para reducir la variabilidad entre curvas asociadas a las diferencias de fase en el caso de movimientos cíclicos.

Otras alternativas más complejas, como el registro de funciones reduce algo más la variabilidad, pero la mejora no compensa la complejidad del método. Además, al registrar las derivadas las funciones primitivas pueden alterarse, lo que implica que no se respeta la dinámica del movimiento. Quizás la utilidad de estas técnicas sea mayor en el caso de funciones claramente no armónicas donde no interesan las derivadas, como en el caso de los registros de fuerzas. Sin embargo, cuando deban analizarse a la vez funciones y sus 
derivadas (como en los estudios de coordinación) deberían utilizarse la normalización lineal o buscar nuevas alternativas si dicha normalización no ofrece resultados satrisfactorios.

\section{Agradecimientos.}

Este trabajo ha sido financiado por el Gobierno de España y cofinanciado por Fondos Feder de la UE gracias al proyecto PDI2017-84201-R (IMBIO3R).

\section{Bibliografía}

BAYDAL-BERTOMEU, J. M., PAGE, Á. F., BELDA-LOIS, et al. (2011). "Neck motion patterns in whiplash-associated disorders: quantifying variability and spontaneity of movement". Clinical Biomechanics, vol. 26, issue 1, pp. 29-34.

BOUlgOURIS, N. V., PLATANIOTIS, K. N., \& HATZINAKOS, D. (2006). "Gait recognition using linear time normalization”. Pattern Recognition, vol. 39, issue 5, pp. 969-979.

CÁCERES, M.L. (2019). "Aplicación de la cinemática articular a la valoración funcional del hombro". Tesis Doctoral. Universitat Politécnica de Valencia.

CRANE, E. A., et al. (2010). "Effect of registration on cyclical kinematic data". Journal of biomechanics, vol 43, issue 12, pp 2444-2447.

CRANE, E., CHILDERS, D., GERSTNER, G., \& ROTHMAN, E. (2011). "Functional data analysis for biomechanics". In: Theoretical Biomechanics. IntechOpen.

EFRON, B., \& TIBSHIRANI, R. J. (1994). An introduction to the bootstrap. CRC press.

LIEBL, D., MOSLER, K., \& WILLWACHER, S. (2012). "Robust clustering of joint moment curves". Sportinformatik, pp 68.

LUCERO, J. C., \& KOENIG, L. L. (2000). "Time normalization of voice signals using functional data analysis". The Journal of the Acoustical Society of America, vol. 108, issue 4, pp. 1408-1420.

MECHMECHE, I., MITICHE, A., OUAKRIM, Y., et al. (2016). "Data correction to determine a representative pattern of a set of 3D knee kinematic measurements". In: 2016 38th Annual International Conference of the IEEE Engineering in Medicine and Biology Society (EMBC), pp. 884-887). IEEE.

NOVACHECK, T. F. (1998). "The biomechanics of running”. Gait \& posture, vol 7, issue 1, pp. 7795.

PAGE, A., \& EPIFANIO, I. (2007). "A simple model to analyze the effectiveness of linear time normalization to reduce variability in human movement analysis". Gait \& posture, vol. 25, issue1, pp. 153-156. 
Comparación de la normalización lineal de la escala de tiempos con el registro funcional continuo en movimientos cíclicos del cuello

PAGE, A., AYALA, G., LEON, M. T.,et al. (2006). "Normalizing temporal patterns to analyze sit-tostand movements by using registration of functional data". Journal of biomechanics, vol. 39, issue 13, pp. 2526-2534.

RAMSAY, J. O., \& LI, X. (1998). "Curve registration". Journal of the Royal Statistical Society: Series B (Statistical Methodology), vol 60, issue 2, pp. 351-363.

RAMSAY, J. O., \& SILVERMAN, B. W. (2007). "Applied functional data analysis: methods and case studies". Springer.

SNOOK, S. H., \& CIRIELLO, V. M. (1991). "The design of manual handling tasks: revised tables of maximum acceptable weights and forces”. Ergonomics, vol. 34, issue 9, pp. 1197-1213.

SØRENSEN, H., GOLDSMITH, J., \& SANGALLI, L. M. (2013). "An introduction with medical applications to functional data analysis". Statistics in Medicine, vol 32 issue 30, pp. 5222-5240.

ULLAH, S., \& FINCH, C. F. (2013). "Applications of functional data analysis: A systematic review". BMC Medical Research Methodology, 13(1), 43.

WINTER; D.A. (1991). "The biomechanics and motor control of human gait : normal, elderly and pathological”. University of Waterloo Press. Waterloo. 


\title{
Validación de los marcadores ArUco para el análisis de movimientos humanos
}

\author{
Jara, Nicolás ${ }^{a}$; Abad, José Luis ${ }^{b}$; Navarro, Joséc; Page, Álvaro ${ }^{\text {d* }}$ \\ ${ }^{a}$ ETSI Industriales, Universitat Politècnica de València (España), nijaget@etsii.upv.es, \\ ${ }^{b}$ ETSI Telecomunicación, Universitat Politècnica de València, joabos@ade.upv.es, \\ ${ }^{\mathrm{c}}$ Dpto. de Sistemas Informáticos y Computación, Universitat Politècnica de València, \\ jonaal1@doctor.upv.es, \\ ${ }^{\mathrm{d}}$ Instituto Universitario Mixto de Biomecánica, Universitat Politècnica de València, \\ afpage@ibv.upv.esc
}

\begin{abstract}
Although video-photogrammetry is the gold standard for human movement analysis, its complexity and high cost arouse interest in simpler and cheaper alternatives. One of them is videoanalysis, which allows analyzing movements in a plane. An alternative that allows the study of movements in $3 D$ is the uaugmented reality markers (AR markers), widely used in the field of robotics. These systems allow to analyze, in real time and with a single video camera, the position and orientation of objects with sufficient precision for use in many biomechanical applications. This article analyzes the accuracy of ArUco markers in the measurement of angles and displacements, comparing the movements measured with the system with two precision techniques: encoders of linear and angular displacements and a standar equipment of videophotogrammetry. The results show that markers can measure displacements with errors lower than those associated with human variability, so it would be possible to use this type of markers in a wide variety of biomechanical applications.
\end{abstract}

Keywords: Human movement, video analysis, OpenCV, ArUco, accuracy.

\section{Resumen}

Aunque la videofotogrametría es la técnica de referencia para el análisis de movimientos humanos, su complejidad y elevado coste hacen que se planteen otras alternativas más sencillas y baratas. Una de ellas es el videoanálisis, que permite analizar movimientos en un plano. Una alternativa que permite el estudio de movimientos $3 D$ es el uso de marcadores de realidad aumentada (AR), de amplio uso en el campo de la robótica. Estos sistemas permiten analizar, en tiempo real y con una única cámara de video, la posición y orientación de objetos con precisión suficiente para su uso en muchas aplicaciones biomecánicas. En este trabajo se analiza la precisión de los 
marcadores ArUco en la medida de ángulos y desplazamientos, comparando los movimientos medidos con el sistema con dos técnicas de precisión: encoders de desplazamientos lineales y giros, para las medidas estáticas, y un sistema de videofotogrametría de precisión, para las medidas en movimiento. Los resultados muestran que los marcadores pueden medir desplazamientos con errores inferiores a los asociados a la variabilidad humana, por lo que sería posible utilizar este tipo de marcadores para numerosas aplicaciones biomecánicas.

Palabras clave: Análisis de movimientos humanos, videoanálisis, marcadores de realidad virtual, ArUco, OpenCV, precisión.

\section{Introducción}

Aunque la videofotogrametría es la técnica de referencia para el análisis de movimientos humanos, su complejidad y elevado coste hacen que se planteen otras alternativas más sencillas y baratas En los últimos años se han publicado numerosos trabajos sobre el empleo de técnicas de bajo coste para el análisis de movimientos humanos, especialmente mediante sensores inerciales. Aunque estos sensores pueden proporcionar una buena estimación de los ángulos articulares, no permiten la medida de desplazamientos con suficiente precisión (Picerno, 2017).

Una alternativa es el uso del videoanálisis, que se ha centrado en el desarrollo de sistemas simples para el análisis de movimientos con una sóla cámara. En esta línea se han desarrollado diferentes sistemas sencillos, pero restringidos al análisis de movimientos planos, lo que limita las posibles aplicaciones (Otín et al., 2016).

Sin embargo, en el campo de la robótica se vienen usando desde hace tiempo sistemas de posicionado mediante marcadores de realidad aumentada (AR). Estos sistemas permiten analizar, en tiempo real y con una única cámara de video, la posición y orientación de objetos con precisión suficiente para su uso en muchas aplicaciones biomecánicas (Parrilla et al., 2013). En particular, la librería ArUco (Muñoz-Salinas, 2012) permite analizar movimientos de varios marcadores de manera rápida y eficiente, por lo que se han aplicado al control de robots colaborativos (Maule et al., 2017).

Sin ambargo, a pesar de su potencial, este tipo de marcadores apenas han sido utilizados en el ámbito de la Biomecánica o en aplicaciones clínicas, por lo que no conocemos su precisión y fiabilidad, si bien los pocos estudios publicados muestran las posibilidades de este sistema (Nagymáté and Kiss, 2019).

En esta línea se plantea el presente trabajo, en el que se analiza la precisión de los marcadores 
ArUco en la medida de ángulos y desplazamientos, comparando los movimientos medidos con el sistema con dos técnicas de precisión: encoders de desplazamientos lineales y giros, para las medidas estáticas, y un sistema de videofotogrametría de precisión, para las medidas en movimiento.

\section{Material y métodos}

\subsection{Marcadores ArUco. Instrumentación utilizada}

La librería ArUco fue desarrollada originalmente por Rafael Muñoz y Sergio Garrido en la Universidad de Córdoba. Está basado en OpenCV y permite la detección de varios tipos de etiquetas. Su proceso de detección es mucho más efectivo y rápido que otras librerías, lo que lo hace especialmente adecuado para aplicaciones del análisis de movimientos en tiempo real. Los marcadores ArUco, son marcadores cuadrados compuestos por un borde negro ancho con una matriz binaria interna. El borde negro permite una rápida detección de la imagen y la codificación binaria permite la identficación de marcadores, la aplicación de técnica de detección y corrección de errores (Garrido-Jurado et al, 2014)

El proceso de detección de un marcador se realiza en dos etapas: la detección de candidatos a marcadores y el análisis de codificación. En el proceso de detección, se lleva a cabo una extracción de contornos y un filtrado, junto con el umbral adaptativo, para detectar formas cuadradas. En la segunda etapa, se analiza la codificación interna, mediante la cual se determina si el marcador pertenece a un tipo de etiquetas específico. La posición de las cuatro esquinas del marcador en la imagen y el tamaño real del marcador son suficientes para que el sistema pueda estimar su posición completa en escala absoluta.

La aplicación desarrollada puede detectar hasta 6 marcadores diferentes. Para cada uno de ellos se proporciona como salida el instante de tiempo, la identificación del marcador, la posición del centro del marcador y la orientación del sistema de referencia ligado al marcador, expresada como el vector giro respecto al sistema de referencia de la cámara medido en radianes. Los vectores están expresados en la base del sistema de referencia de la cámara.

La velocidad de adquisición depende de la cámara usada, del ordenador y del número de marcadores. En este trabajo se ha usado una frecuencia de 25 fotogramas por segundo.

\subsection{Aplicación al análisis de movimientos humanos. Dispositivos y análisis cinemático}

Para la captura de movimientos se ha usado una cámara Logitech modelo C920 Pro. La 
cámara se calibra según el procedimiento descrito en el software usando un patrón de cuadrículas.

Para capturar el movimiento no se ha usado un solo marcador por segmento, sino un sistema formado por dos marcadores de $7 \mathrm{~cm}$ de lado que forman un pequeño ángulo (unos $20^{\circ}$ ) (Figura 1). La razón para usar dos marcadores es doble. Por una parte, porque mejora la precisión de las medidas, al usar información redundante. El efecto sobre el error de desplazamientos lineales es una mejora por un factor $\sqrt{ } 2$. La mejora sobre el error en los ángulos depende de la separación entre marcadores y es superior a ese factor (Page, et al, 2009). Por otra, se evita una discontinuidad en el registro de la orientación que aparece cuando un marcador queda paralelo al plano de la cámara o casi paralelo; en este caso hay una indeterminación en la orientación, presentándose discontinuidades en el ángulo y con un aumento notable de los errores. Este problema se evita con dos marcadores que no sean paralelos, ya que siempre habrá al menos un marcador no paralelo a la cámara.

La información sobre la posición y orientación del sistema de marcadores se realiza asignando a cada marcador 4 puntos virtuales, correspondientes al origen del sistema local del marcador y a puntos situados sobre los ejes a una distancia de $10 \mathrm{~cm}$ del origen. Esto equivale a disponer de 8 marcadores técnicos por cada segmento. El análisis cinemático se realiza entonces con los algoritmos descritos en Page et al. (2009), obteniéndose el vector de Rodrigues y el desplazamiento del sistema respecto de una posición inicial de referencia.

\subsection{Validación del sistema de medida}

Para validar el sistema de medida se han realizado dos experimentos, destinados a establecer la validez en la medida de desplazamientos estáticos y en movimiento.

El primer experimento tenía como objetivo determinar el error de medida en desplazamientos estáticos, y analizar el efecto de parámetros experimentales como la distancia del marcador a la cámara, separación del eje o inclinación. Para ello se han usado los montajes que se muestran en la Figura 1, donde el sistema de dos marcadores se desplaza sobre la guía de un encoder lineal o gira ligado a un ángulo. Los encoders son marca Digital Protactor, con una resolución de $0.01 \mathrm{~mm}$ y 0.1 , respectivamente. La incertidumbre de los encoders es de 0.1 $\mathrm{mm}$ y $0.3^{\circ}$.

En el caso de los desplazamientos lineales, se han desarrollado dos experimentos, colocando el encoder paralelo al eje $\mathrm{Z}$ de la cámara (profundidad) o perpendicular al mismo. El sistema se ha ido moviendo desde una posición de referencia en intervalos aproximados de $2 \mathrm{~cm}$ y se han contrastado las diferenciss en el desplazamiento medidas con el encoder y las proporciandas por los marcadores ArUco. Se han realizado repeticiones a diferentes distancias a la cámara $(60 \mathrm{~cm}, 75 \mathrm{~cm}, 100 \mathrm{~cm})$. 


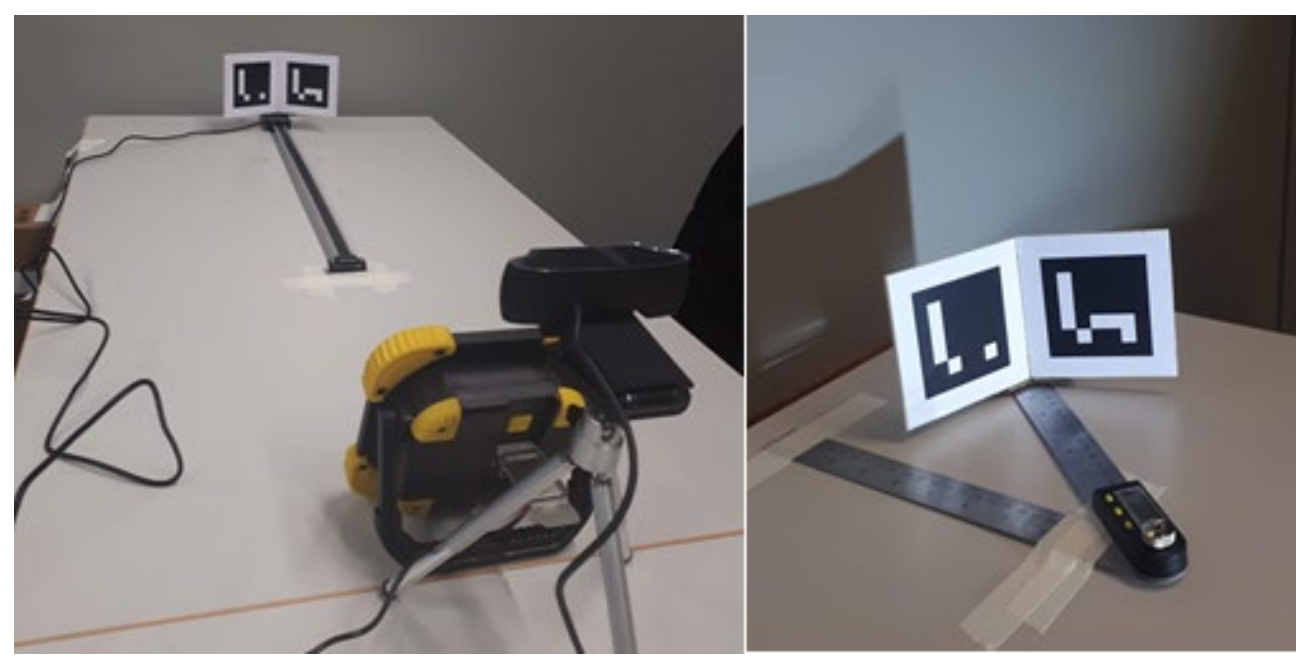

Fig. 1. Montajes para el análisis de los errores en los desplazamientos lineales y angulares estáticos

En el caso de los desplazamientos angulares, se fijó el sistema de marcadores a uno de los brazos del enconder angular y se midieron desplazamientos angulares de hasta $50^{\circ}$ en dos situaciones: con el eje de giro paralelo al eje de la cámara y con el eje de giro del encoder paralelo al plano de la cámara.

En ambos casos se compararon las medidas del sistema ArUco y de los encoders y se obtuvieron dos parámetros de concordancia: el coeficiente de correlación intraclase (ICC) y el error estándar de las medidas (SEM). El ICC se calculó tal como de describe en Weir (2005), usando el tipo ICC(3), al ser el factor "método de medida" un factor fijo. En cuanto al SEM, se ha calculado como la raíz cuadrada del error cuadrático medio en $\mathrm{n}$ anova de dos vías (Weir, 2005).

En el segundo experimento se han comparado las medidas angulares en movimiento, usando una tabla con una bisagra cuyo eje se coloca paralelo al plano de la cámara o perpendicular. Sobre la tabla se fija un sistema de dos marcadores ArUco y un conjunto de marcadores reflectantes. La tabla se movió en un rango aproximado de $50^{\circ}$, a una velocidad normal en análisis de movimientos humanos $(0.5 \mathrm{~Hz})$. El movimiento de la tabla se registró usando los marcadores ArUco y registrando el movimiento con un sistema de videofotogrametría Kinescan-IBV, calibrado para un precisión de $0.3 \mathrm{~mm}$ en la medida de posición y de $0.2^{\circ}$ en la medida de ángulos (Figura 2.).

Al igual que en el caso anterior, la concordancia en cada movimiento se describió mediante el ICC y el SEM. 


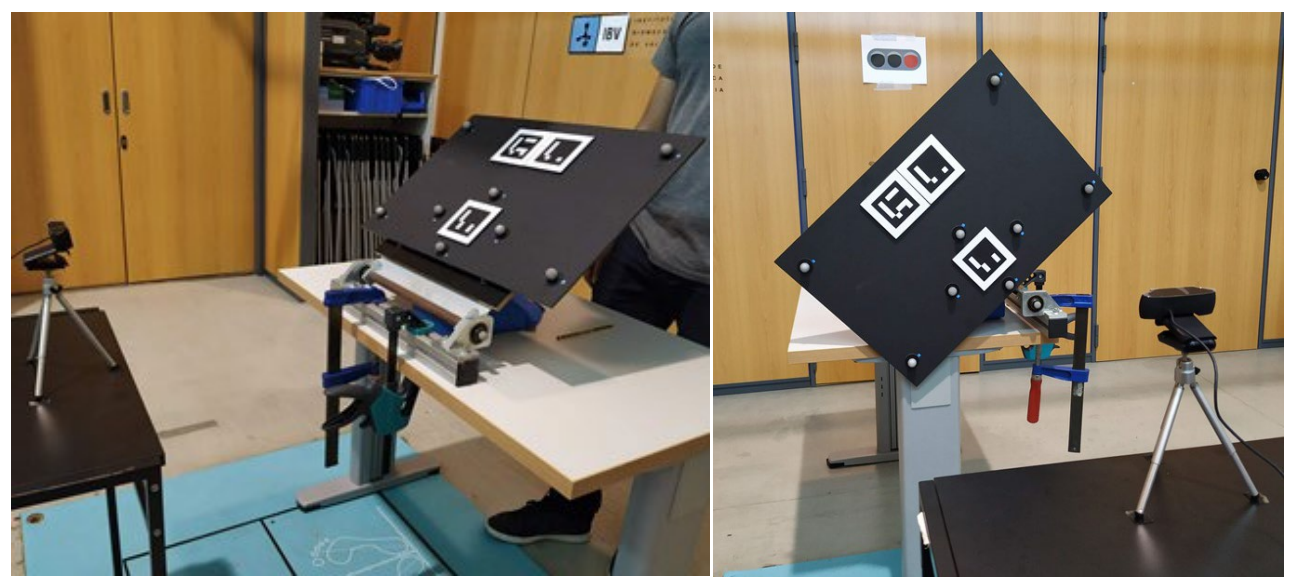

Fig. 2. Comparación entre el movimiento registrado por un sistema e fotogrametría y los marcadores ArUco. El movimiento del sistema ArUco se registra exclusivamente con los dos marcadores en la parte superior de la tabla. El marcador inferior se usa para definir un sistema de referencia común a la cámara de vídeo y al sistema de videofotogrametría.

\section{Resultados y Discusión}

La tabla 1 muestra los valores del ICC entre las medidas con encoders y ArUco, así como el SEM obtenido, para los desplazamientos lineales y angulares estáticos.

Como puede observarse, la medida de desplazamientos estáticos con ArUco es muy precisa. Los ICC tienen valores superiores a 0.999 en todos los casos, lo que muestra una concordancia excelente.

El error asociado a los giros con el eje perpendicular a la cámara es del orden de la precisión del encoder $\left(\mathrm{SEM}=0.2^{\circ}\right)$, mientras que los giros en un eje paralelo a la cámara tienen un error algo mayor ( $\mathrm{SEM}=0.8^{\circ}$ ). Dicha diferencia se debe a que en el segundo caso hay un desplazamiento en profundidad, que se mide con menos precisión con una sóla cámara. Esto indica que en caso de movimientos humanos, la cámara debería colocarse paralela al plano principal del movimiento (por ejemplo, plano sagital en el caso de marcha humana). En cualquier caso, se trata de un error de medida mucho más pequeño que el de otros dispositivos usados en el ámbito clínico, como los inclinómetros y goniómetros que tienen errores del orden de $3^{\circ}$ (Audette et al, 2010). Por otra parte, la variabilidad humana también es muy superior a estos valores. Por ejemplo, en el estudio del rango de flexo-extensión del cuello, la variabilidad de los sujetos al repetir la prueba tiene un SEM del orden de 5.5 (Cagnie et al., 2007). Por tanto, el efecto del pequeño error de medida de este sistema es despreciable frente a las diferencias de comportamiento del mismo sujeto en pruebas diferentes. 
Tabla 1. Concordancia entre las medidas de desplazamientos angulares y lineales estáticos, medido con encoder y con marcadores ArUco

\begin{tabular}{|c|c|c|c|}
\hline & & $\begin{array}{l}\text { Coeficiente de } \\
\text { correlación intraclase } \\
\text { (ICC). Adimensional }\end{array}$ & $\begin{array}{c}\text { Error estandar de la } \\
\text { medida (Giros en }{ }^{\circ} ; \\
\text { desplazamientos en } \mathbf{m m} \text { ) }\end{array}$ \\
\hline \multirow[t]{2}{*}{ Giro } & $\begin{array}{l}\text { Eje paralelo a la } \\
\text { cámara }\end{array}$ & 0,9996 & 0,80 \\
\hline & $\begin{array}{c}\text { Eje perpendicular } \\
\text { a la cámara }\end{array}$ & 1,0000 & 0,20 \\
\hline \multirow[t]{5}{*}{ Desplazamiento } & $\begin{array}{l}\text { Paralelo cámara, } \\
\mathrm{Z}=100 \mathrm{~cm}\end{array}$ & 0,9998 & 1,50 \\
\hline & $\begin{array}{l}\text { Paralelo cámara, } \\
\qquad \mathrm{Z}=75 \mathrm{~cm}\end{array}$ & 0,9999 & 1.00 \\
\hline & $\begin{array}{l}\text { Paralelo cámara, } \\
\qquad \mathrm{Z}=60 \mathrm{~cm}\end{array}$ & 0,9999 & 1,20 \\
\hline & $\begin{array}{c}\text { Paralelo al eje de } \\
\text { la cámara, } \\
\text { desplazado } \\
\text { lateralmente }\end{array}$ & 0,9993 & 3,40 \\
\hline & $\begin{array}{l}\text { Paralelo al eje de } \\
\text { la cámara, } \\
\text { centrado }\end{array}$ & 0,9999 & 1.10 \\
\hline
\end{tabular}

En cuanto a los desplazamientos lineales, el error más grande aparece cuando los desplazamientos son en profundidad (eje $\mathrm{Z}$ de la cámara) y además están separados de dicho eje. En este caso los errores son del orden de $3 \mathrm{~mm}$. Por el contrario, el hecho de centrar el eje mejora notablemente la concordancia, obteniéndose un error el orden de $1 \mathrm{~mm}$. Estos resultados indican la conveniencia de centrar bien el campo de medida, de manera que los marcadores aparezcan en la zona central de la imagen.

En el caso de los desplazamientos paralelos al plano de la cámara, el error depende de la separación a la cámara, lo que resulta previsible, dado que al separar los marcadores aparecen más pequeños en la imagen y aumenta el error aleatorio (Page et al., 2008). Para el tamaño de marcador de este estudio, las medidas tienen errores del orden de $1 \mathrm{~mm}$ hasta una distancia de $0.75 \mathrm{~m}$. A distancias mayores, el error crece. No obstante, para las distancias focales que se manejan en las cámaras web esa distancia es suficiente para muchas aplicaciones, por ejemplo para analizar el miembro inferior o el movimiento de la cabeza. Por ello, y al igual que sucede con cualquier sistema de visión por computador, la precisión mejora si se ajusta 
el campo de visión a la zona de movimiento real.

Tabla 2. Concordancia entre las medidas de desplazamientos angulares dinámicos, medido con videofotogrametría y con marcadores ArUco

Coeficiente de correlación intraclase

(ICC). Adimensional

\author{
Error estandar de la \\ medida $\left({ }^{\circ}\right)$
}

\begin{tabular}{ccc}
\hline Giro & $\begin{array}{c}\text { Paralelo al eje de } \\
\text { la cámara }\end{array}$ & 0,9970 \\
$\begin{array}{c}\text { Paralelo a la } \\
\text { cámara }\end{array}$ & 0.9960 & 1.70 \\
& & 1.20 \\
\hline
\end{tabular}

En cuanto a la precisión en la medida de ángulos en movimiento, los resultados aparecen en la Tabla 2. Aunque los ICC siguen siendo excelentes, el error angular es superior a $1^{\circ}$ tanto en los giros respecto de un eje paralelo al eje de la cámara $\left(\mathrm{SEM}=1.20^{\circ}\right)$, como en un eje paralelo a la cámara $\left(\mathrm{SEM}=1.7^{\circ}\right)$. Estos resultados presentan errores mayores que los obtenidos por Parrilla et al (2013), si bien las diferencias pueden deberse al tipo de movimiento analizado en dicho trabajo, que estaba confinado en un espacio más pequeño, o a los algoritmos de cálculo que en nuestro estudio se realizan en tiempo real. Por el contrario, los errores obtenidos en nuestro trabajo son bastante menores que los descritos por Nagymate et al. (2019) en un estudio sobre marcha. En nuestra opinión, los pobres resultados mostrados en dicho estudio se deben más a la presencia de artefactos por movimiento de tejidos blandos que a la precisión del equipo.

En cualquier caso se trata de errores angulares muy pequeños y asumibles en muchas aplicaciones, como la medida de rangos de movimiento de la cabeza o brazo. Como se ha indicado anteriormente, la variabilidad intrasujeto es mayor, en muchos casos a este nivel de errores.

\section{Conclusiones}

A la vista de los resultados obtenidos en este trabajo, cabe concluir que los marcadores ArUco son una herramienta de gran potencial en el desarrollo de sistemas de análisis de movimientos para aplicaciones clínicas. No sólo permite hacer mediciones en tiempo real y con un coste muy bajo (una webcam de calidad cuesta menos de 100 euros), sino que ofrece niveles de precisión suficientes. Así, los errores obtenidos son bastante menores que los asociados a la 
variabilidad humana y menores que los de otros equipos como inclinómetros. Es un sistema libre de derivas y sin problemas a la hora de interpretar la posición de los sistemas de referencia

Sin embargo, la calidad de las medidas depende del diseño del sistema experimental, que debe verificar algunas condiciones, como estar centrado en el centro de la imagen y con un campo bien ajustado a la amplitud del movimiento.

\section{Agradecimientos}

Este trabajo ha sido financiado por el Gobierno de España y cofinanciado por Fondos Feder de la UE gracias al proyecto PDI2017-84201-R (IMBIO3R).

\section{Referencias}

AUDETTE, I., DUMAS, J. P., COTE, J. N., \& DE SERRES, S. J. (2010). « Validity and between-day reliability of the cervical range of motion (CROM) device». Journal of Orthopaedic \& Sports PhysicalTtherapy, vol. 40, issue 5, pp. 318-323.

CAGNIE, B., COOLS, A., DE LOOSE, V., CAMBIER, D., \& DANNEELS, L. (2007). « Reliability and normative database of the Zebris cervical range-of-motion system in healthy controls with preliminary validation in a group of patients with neck pain». Journal of Manipulative and Physiological Therapeutics, vol. 30, issue 6, pp. 450-455.

GARRIDO-JURADO, S., MUÑOZ-SALINAS, R., MADRID-CUEVAS, F. J., \& MARIN-JIMENEZ, M. J. (2014). «Automatic generation and detection of highly reliable fiducial markers under occlusion », Pattern Recognition, vol. 4, issue 6, p,2280-2292.

MAULE, L., FORNASER, A., TOMASIN, P., TAVERNINI, M., MINOTTO, G., DA LIO, M., \& DE CECCO, M. (2017). « Augmented robotics for electronic wheelchair to enhance mobility in domestic environment ». In : International Conference on Augmented Reality, Virtual Reality and Computer Graphics (pp. 22-32). Springer, Cham.

MUÑOZ-SALINAS, R. (2012). «Aruco: a minimal library for augmented reality applications based on OpenCV ». Universidad de Córdoba.

NAGYMATE, G., \& KISS, R. M. (2019). «Affordable gait analysis using augmented reality markers ». PloS one, vol. 14, issue 2, e0212319.

OTIN, C. (2016). «Análisis de habilidades deportivas mediante el uso del software Kinovea ». In Simbiosis del aprendizaje con las tecnologías: experiencias innovadoras en el ámbito hispano (pp. 125134). Prensas Universitarias de Zaragoza.

PAGE, Á., DE ROSARIO, H., MATA, V., \& ATIENZA, C. (2009). « Experimental analysis of rigid body motion. A vector method to determine finite and infinitesimal displacements from point coordinates ». Journal of Mechanical Design, vol. 131, issue 3, pp. 031005. 
PAGE, A., MORENO, R., CANDELAS, P., \& BELMAR, F. (2008). «The accuracy of webcams in 2D motion analysis: sources of error and their control ». European Journal of Physics, vol. 29, issue 4, pp. 857.

PARRILLA, E., et al.. (2013). « Ankle 3D-kinematics measurement by using a single camera and ARmarkers ». Footwear Science, vol. 5, sup.1, pp. 73-74.

PICERNO, P. (2017). "25 years of lower limb joint kinematics by using inertial and magnetic sensors: a review of methodological approaches“. Gait \& Posture, vol. 51, p. 239-246.

WEIR, J. P. (2005). «Quantifying test-retest reliability using the intraclass correlation coefficient and the SEM ». The Journal of Strength \& Conditioning Research, 19(1), 231-240. 


\title{
Fundamentos biomecánicos en el diseño de inmovilizador para rehabilitación de fracturas múltiples
}

\section{Bula Oyola, Ena Lucía}

Doctorando en Tecnologías para la Salud y el Bienestar, Universidad Politécnica de Valencia, España, Becario Desarrollo Profesoral, Universidad del Norte, Colombia, enbuoy@doctor.upv.es

\begin{abstract}
Bone pathologies involving the shoulder girdle are common and the rehabilitation is most frequently achieved through conservative treatments. We present the case of 20-year-old female patient with multiple trauma due to high-energy accident. Among the injuries, there was a displaced fracture of the diaphysis of the right clavicle, a displaced fracture of the left humerus, 11 fractures in 8 ribs (2 with displacement) and scapular dyskinesis.

Considering the instability of the thorax and the incompatibility between the fixation "8" (Watson-Jones) and the disposition of the displaced fragments, we designed a fracture immobilizer based on the functional analogy of the shoulder girdle. The external traction system developed emulates the action of the Pectoralis Major, Deltoid, Trapezius, Latissimus Dorsi, the upper area of the Oblique of the Abdomen and Serratus Anterior.
\end{abstract}

The continuous use of the product during six weeks allowed the consolidation of the fractures, restored the stability of the scapular girdle and the range of functional movement. The adequate traction of the fragments of the clavicle and its slight overlap contributed to attenuate the discontinuity in the shoulder.

The main achievements in the proposed design are: double traction effect; posterior fixation to keep the clavicle and shoulder blades in position under dynamic conditions and the possibility of applying pressure in a gradual and independent way in predetermined points. These principles could be applicable in the rehabilitation of other types of multiple fractures.

Keywords: rehabilitation, biomechanics, clinical experiences, exoskeletons, fractures, polytraumatism

\section{Resumen}

Las patologías óseas que afectan a la cintura escapular son comunes y en la mayoría de los casos su recuperación se obtiene a través de tratamiento conservador. Se presenta el caso de paciente femenino de 20 años con politraumatismo por accidente de alta energía. Entre las lesiones se 
encuentran: una fractura diafisiaria de clavícula derecha con desplazamiento, fractura diafisiaria del húmero izquierdo con desplazamiento, 11 fracturas en 8 costillas (2 con desplazamiento) y disquinesia escapular.

Teniendo en cuenta la inestabilidad torácica y la incompatibilidad entre la fijación en " 8 " (Watson-Jones) frente a la disposición de los fragmentos desplazados, se diseñó un inmovilizador basado en la analogía funcional de la cintura escapular y se estableció un sistema de tracciones externos que emulan la acción de los músculos Pectoral Mayor, Deltoides, Trapecio, Dorsal Ancho, la zona superior del Oblicuo Mayor del Abdomen y Serrato Anterior.

El uso continuo del producto durante seis semanas favoreció la consolidación de las fracturas, restituyó la estabilidad de la cintura escapular y el rango de movimiento funcional. La adecuada tracción de los fragmentos de la clavícula y su leve superposición contribuyó a atenuar la discontinuidad en el hombro.

Los principales beneficios en el diseño propuesto son: efecto de doble tracción, fijación posterior para mantener la clavícula y omoplatos en posición y bajo condiciones dinámicas, y la posibilidad de generar presión en forma gradual e independiente en puntos prefijados. Estos principios podrían ser aplicables en la rehabilitación de otro tipo de fracturas múltiples.

Palabras clave: rehabilitación; biomecánica; experiencias clínicas; exoesqueletos; fracturas; politraumatismo

\section{Introducción}

Las fracturas del tercio medio de la clavícula representan aproximadamente el 75\% de todas las fracturas de este hueso (Postacchini, Gumina, De Santis, \& Albo, 2002) y cerca de la mitad de ellas presentan desplazamiento (Robinson, 1998). Por su parte, las fracturas de costilla comprenden el 12\% de todas las fracturas observadas en pacientes (Barnea, Kashtan, Skornick, \& Werbin, 2002), la mayoría se deben a penetración directa o traumatismo cerrado en el tórax (Kuo \& Kim, 2019) y pueden causar contusiones pulmonares, hemotórax y/o neumotórax (Todd et al., 2006). El adecuado tratamiento para fracturas desplazadas es fuente de debate en la literatura actual, algunos estudios intentan establecer parámetros de medición (desplazamiento $>1,5$ o $2 \mathrm{~cm}$ ) que avalen la intervención quirúrgica y otros aluden a la revisión concreta de cada caso que derive en una decisión consciente. 
Con miras a aportar evidencia a la discusión actual acerca del tratamiento conservador de fracturas con desplazamiento, se presenta el caso de una paciente con politraumatismo tratada con éxito a partir del uso de un nuevo modelo de inmovilización inspirado en el sistema de tracción muscular del tronco y cintura escapular.

\section{Presentación de caso}

Paciente femenina de 20 años de edad, que al ir de pasajera en automóvil colisiona con otro vehículo resultando lesionada. Sufrió trauma de cráneo moderado con alteración de conciencia. Obnubilación. Trauma cerrado del tórax que ocasionó hemotórax y neumotórax de un $40 \%$. Fractura de la diáfisis de la clavícula derecha con desplazamiento de los fragmentos. Discinesia escapular. Fractura cerrada de húmero izquierdo cabalgada. Compromiso traumático del Nervio Radial (mano caída). Fractura del 3, 4, 5, 6, 7, 8, 9, 10 arco costal posterior izquierdo con desplazamiento de los fragmentos en algunos arcos costales (Fig. 1). Cirugía practicada: toracostomía de drenaje cerrado y acceso venoso central. Osteosíntesis de húmero, diáfisis con placas y Neurorrafia Radial.
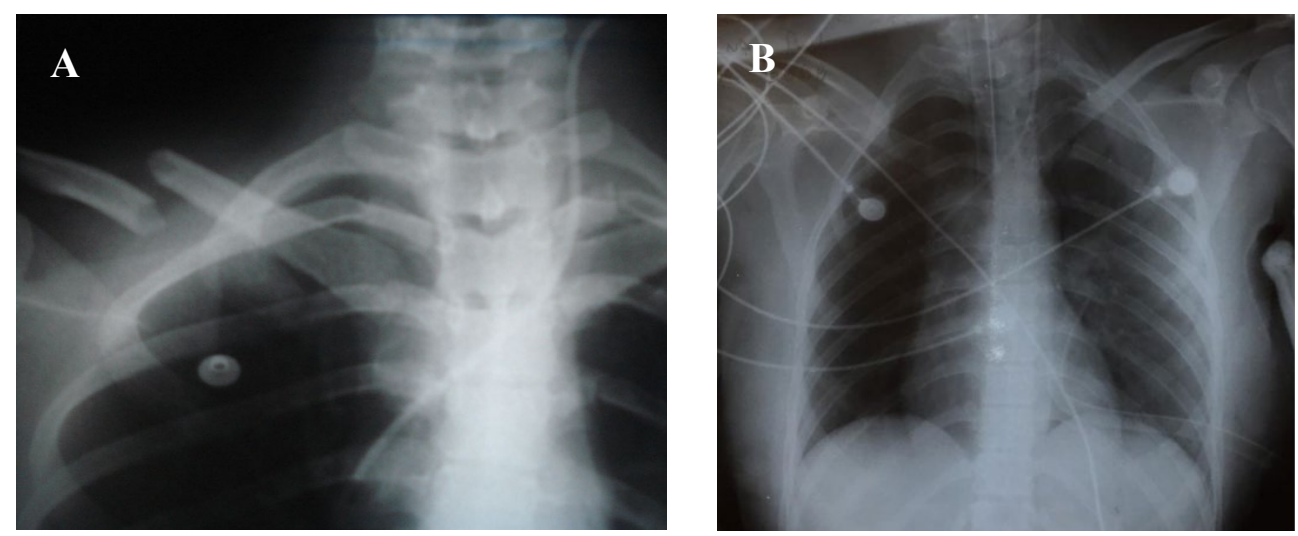

Fig. 1. Radiografías iniciales. (A) Fractura de clavícula. (B) Fracturas de arcos costales, Neumotórax y Hemotórax. Fuente: Clínica Cervantes (2007).

Al egresar de la unidad de cuidados intensivos le fue prescrita una órtesis radial y tratamiento conservador para la rehabilitación de las fracturas restantes, inmovilizador tipo Watson-Jones o "vendaje en 8" para la clavícula y faja para las costillas. 
El primer inmovilizador asignado usaba velcro como método de fijación que coincidía exactamente con la zona axilar, esto obligaba a la paciente a mantener sus brazos en abducción ante la incomodidad del material. En vista de la fluctuación de los fragmentos de la clavícula (síndrome de la tecla de piano) y sobrecarga en el húmero lesionado, optó por usar otro modelo del mismo tipo de inmovilización con fijación a través de hebillas. Con este ejemplar los brazos podían mantenerse en posición anatómica pero los vendajes no lograban presionar el fragmento elevado por acción del músculo esternocleidomastoideo. Posteriormente le recomedaron usar un cabestrillo sin mejores resultados.

El inmovilizador de costillas prescrito consistía en una faja elástica microperforada ajustable con velcro. El producto se situaba alrededor de la región costal inferior y contenía efectivamente los arcos costales inestables pero al menor movimiento se contraía y obligaba a ser fijado reiteradas veces. Esto incrementaba el dolor y la dificultad respiratoria.

Los inconvenientes presentados motivaron el diseño de una alternativa de inmovilización que favoreciera la consolidación de fracturas de clavícula y costillas de manera efectiva y confortable.

\subsection{Biomecánica y diseño}

El diseño del inmovilizador fue concebido por analogía funcional de las tensiones que actúan sobre la clavícula y costillas. La acción de los músculos Pectoral Mayor, Deltoides, Trapecio, Dorsal Ancho, la zona superior del Oblicuo Mayor del Abdomen y Serrato Anterior constituyen un mecanismo anatómico emulable para la estabilización de la cintura escapular y el tórax por medios ortésicos.
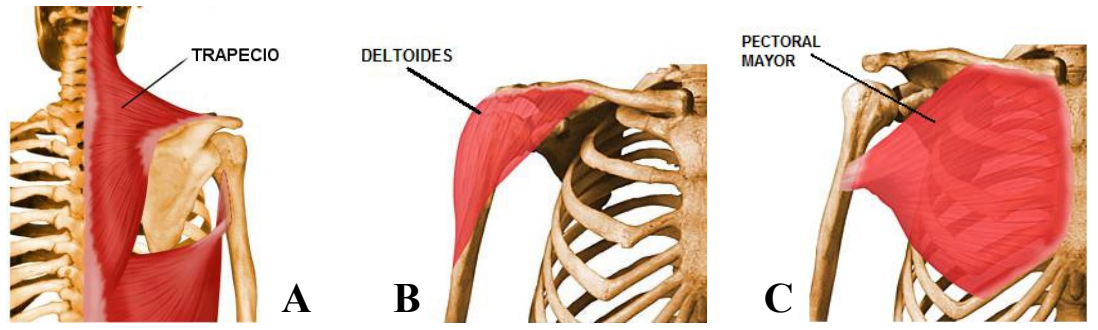

Fig. 2. Músculos Cintura Escapular. (A) Trapecio, (B) Deltoides y (C) Pectoral Mayor.

Fuente: Músculos.org $(n, d)$ 
Se tomó como referencia el Trapecio como estabilizador de la escápula durante los movimientos de la extremidad superior, que por medio de sus fibras horizontales medias tiran de la escápula hacia la columna. El Deltoides como eje de tracción de la zona distal de clavícula hacia el húmero y el Pectoral Mayor como eje de tracción de la zona proximal de la clavícula hacia el tronco (Fig. 2).
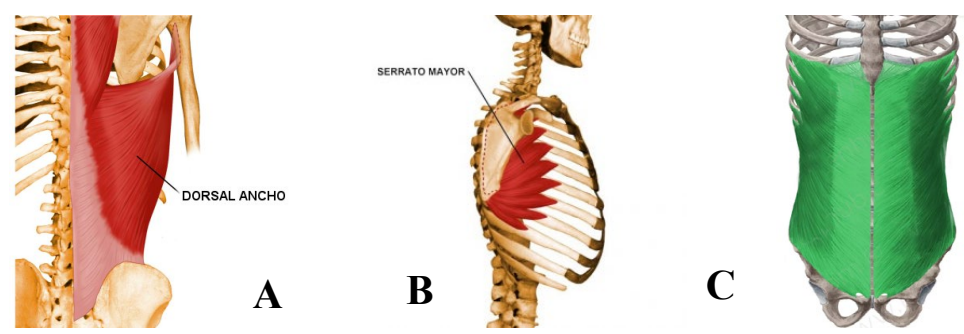

Fig. 3. Músculos de contención de arcos costales. (A) Dorsal Ancho, (B) Serrato Mayor y (C) Oblicuo Mayor del Abdomen. Fuente: Músculos.org $(n, d)$

Asimismo, se identificó la dirección de las tensiones anatómicas de los músculos que contribuyen a la protección de los arcos costales. El Dorsal Ancho que actúa como contención del tórax y el abdomen, el Serrato Anterior que cubre el área lateral del tórax intercalado entre las costillas y la escápula y que en sus fibras inferiores se encuentra con la unión costal del Oblicuo Mayor del Abdomen (Fig. 3).

El inmovilizador de tracción múltiple ó tracción cruzada es una unidad textil compuesta por una solapa que se ubica sobre las clavículas y escápulas ajustándose al tórax a través de dos franjas fijadoras de costillas unidas entre sí a partir de hebillas.
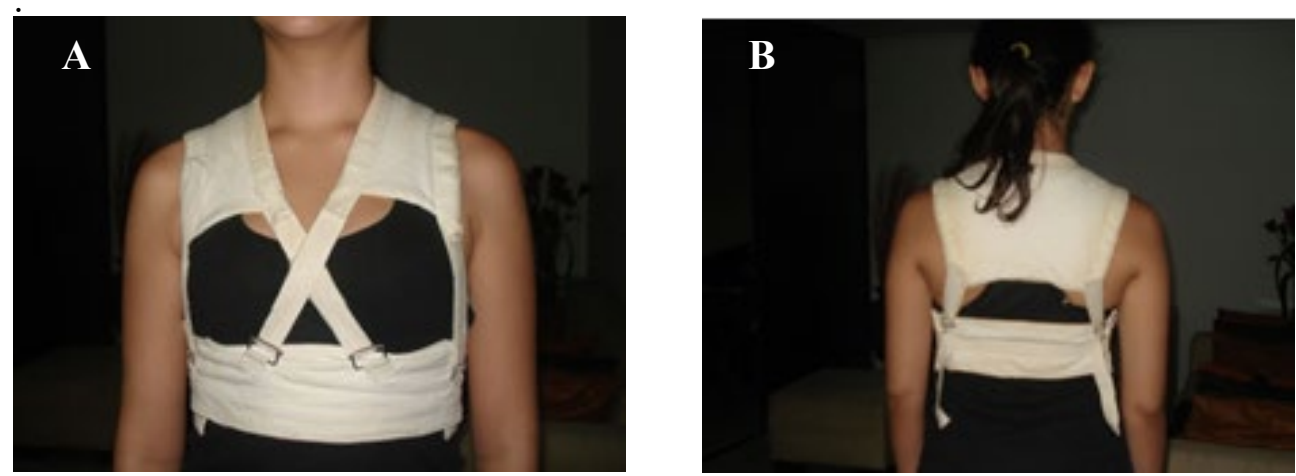

Fig. 4. Inmovilizador de tracción cruzada. (A) Vista frontal y (B) Vista posterior. 
Su configuración en "X" surge de la proyección de las fibras del músculo Pectoral Mayor como mecanismo que favorece el descenso del fragmento proximal de la clavícula, en procura de una posición más cercana a la alineación anatómica natural del hueso. Las franjas de sujeción dispuestas en cada uno de los extremos realizan presión perpendicular sobre el tercio distal y aseguran que la tensión sobre ambos fragmentos sea homogénea. En la zona posterior, la solapa se ajusta al corsé por medio de dos franjas cuyo fin es generar tracción opuesta a las tiras frontales y mantener el tronco erguido. La faja se ajusta mediante velcro y hebillas manteniendo los arcos costales en posición (Fig. 4).

\section{Resultados}

La paciente utilizó el inmovilizador diseñado durante 6 semanas. Durante el período de uso recibió sesiones de terapia respiratoria y posteriormente inició el programa de ejercicios para el fortalecimiento de la cintura escapular. A los 3 meses de seguimiento las fracturas habían consolidado.

\subsection{En función de su congruencia anatómica}

El abultamiento propio de la elevación del tercio proximal de la clavícula difícilmente recibe una presión adecuada que provoque su reducción a través del uso del inmovilizador en "8" (Fig. 5). El inmovilizador de tracción cruzada mantuvo una presión homogénea a lo largo del hueso. Sus tres puntos de presión con direcciones contrarias (hacia el esternón, la escápula y las costillas) restringieron en mayor medida el movimiento de los fragmentos y favorecieron la consolidación de la fractura (Fig.6).
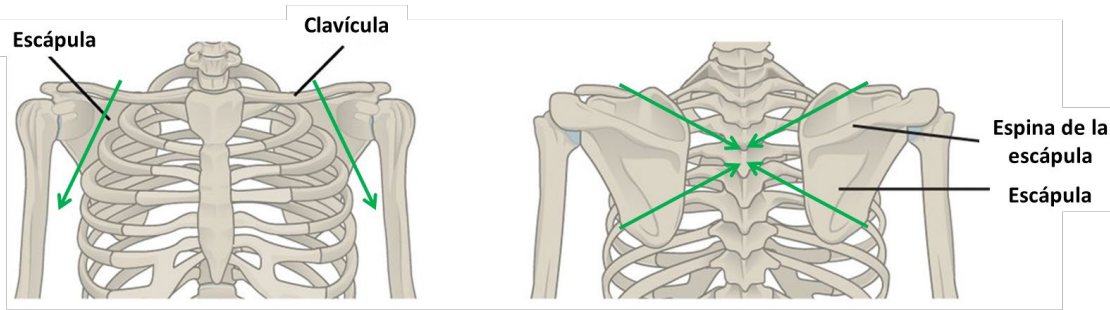

Fig. 5. Distribución de las tensiones sobre los fragmentos del "vendaje en 8 ". Fuente: OpenStax CNX CC 4.0 (2013). 
Bula, E.

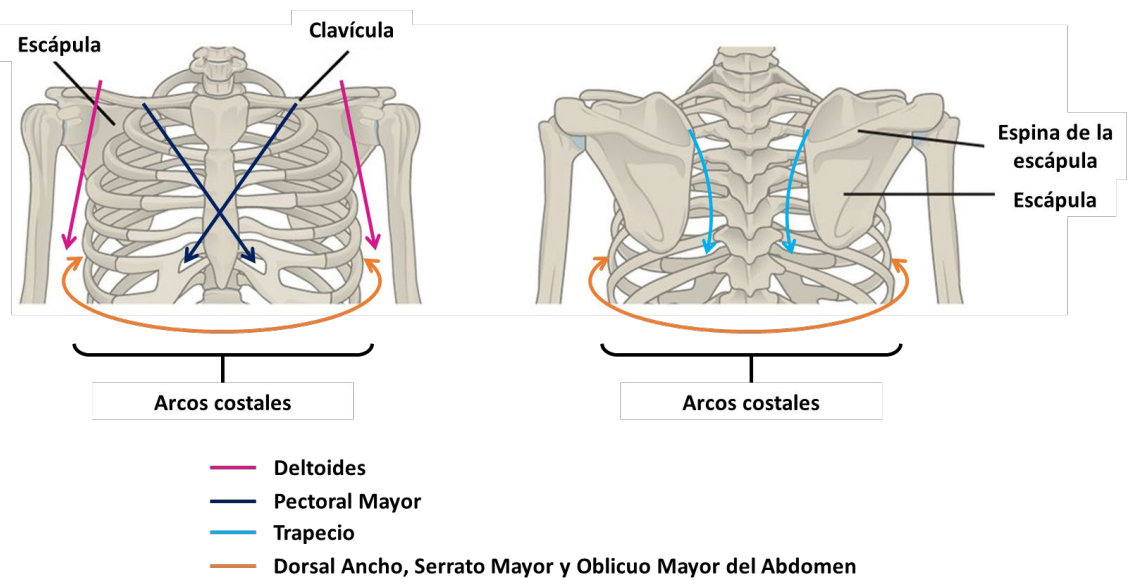

Fig. 6. Distribución de las tensiones sobre los fragmentos del inmovilizador de tracción cruzada. Fuente: OpenStax CNX CC 4.0 (2013).

La faja permitió contener los arcos costales de manera efectiva. El material semirrígido favoreció la estabilidad y su fijación a través de velcro y hebillas aseguró el ajuste, brindando una breve tolerancia para los movimientos respiratorios.

\subsection{En función de su uso}

El inmovilizador de tracción cruzada presenta una estructura similar a un corsé postural. La tensión que ejercen sus franjas posteriores hacia las escápulas favoreció la posición erguida del cuerpo y la reubicación de los fragmentos en dirección anteroposterior. Su configuración limitó los movimientos de elevación, protracción y retracción de la cintura escapular; así como la flexión y extensión del tronco sin interferir con otras zonas del cuerpo, proporcionando mayor comodidad e independencia.

\subsection{En función de la consolidación de las fracturas}

El proceso de rehabilitación se llevó a cabo con una considerable disminución de los síntomas dolor y alta correspondencia con los ejercicios respiratorios. Los fragmentos recibieron un correcto posicionamiento, a las 3 semanas se pudo notar que la evidente inestabilidad torácica había disminuido. A las 6 semanas de 
seguimiento se corroboró la consolidación total a través de exploración radiológica (Fig. 7).
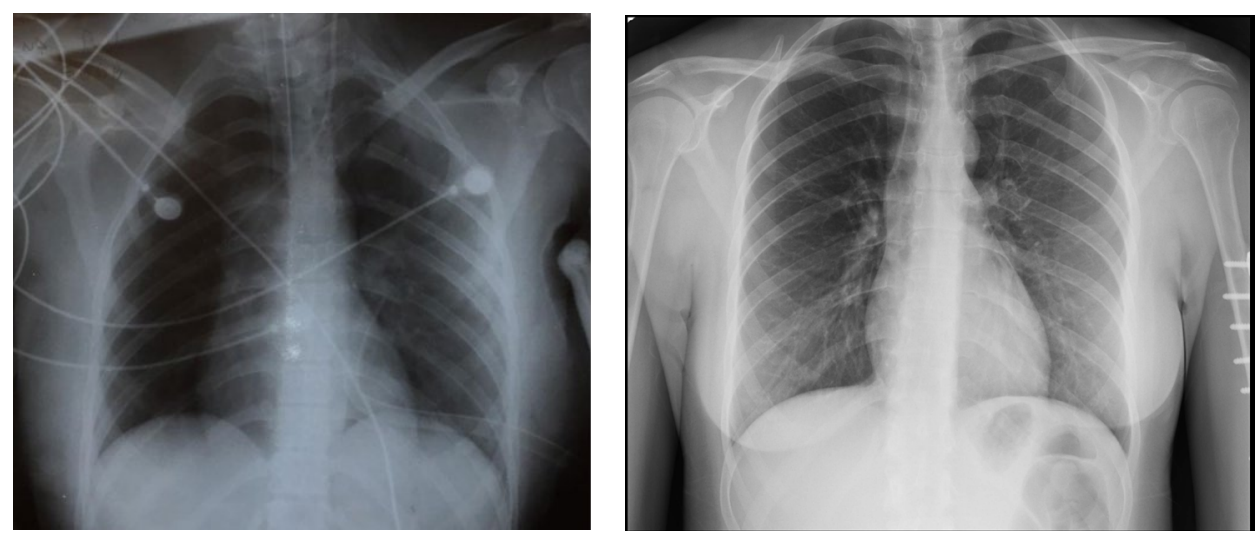

Fig. 7. . Evolución de las fracturas de costillas. (A) Radiografia inicial y (B) Radiografia posterior postratamiento con inmovilizador de tracción cruzada.
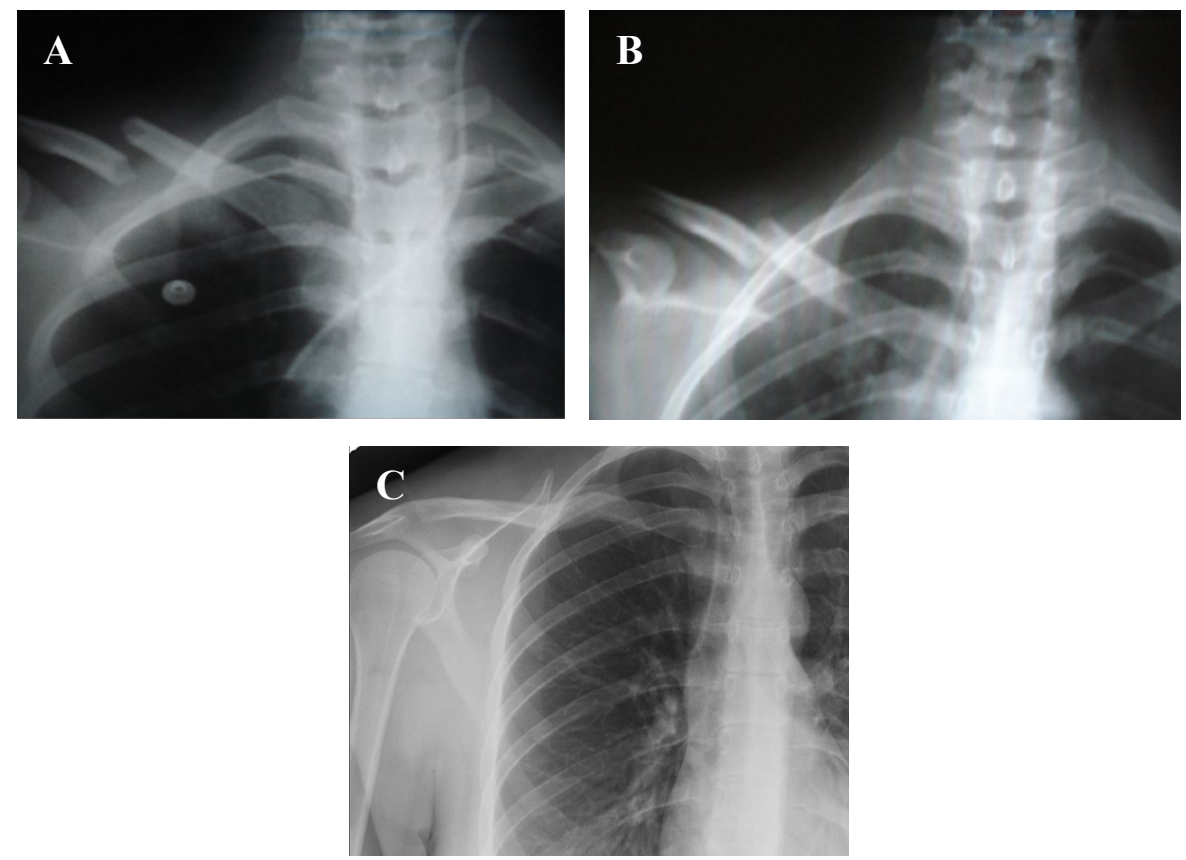

Fig. 8. Evolución de la fractura de clavícula. (A) Radiografia inicial y (B) Radiografía postratamiento seguimiento 3 meses (C) Radiografia posterior consolidación total. 
La rehabilitación de la clavícula se obtuvo en condiciones confortables. Al segundo día de uso, se identificó que el fragmento proximal había descendido completamente y se mantuvo en la misma posición durante las fases posteriores. Hubo una considerable disminución del dolor y funcionalidad del brazo derecho. A las 6 semanas de seguimiento se comprobó la consolidación con una notoria reducción del grado de desplazamiento (Fig. 8).

La reubicación temprana de los fragmentos logró minimizar considerablemente el nivel de acortamiento y volumen óseo. Rango de movilidad y fuerza conservados. La constitución física de la zona mantiene una apariencia cercana a la disposición natural del hueso, la alteración es inapreciable (Fig. 9).

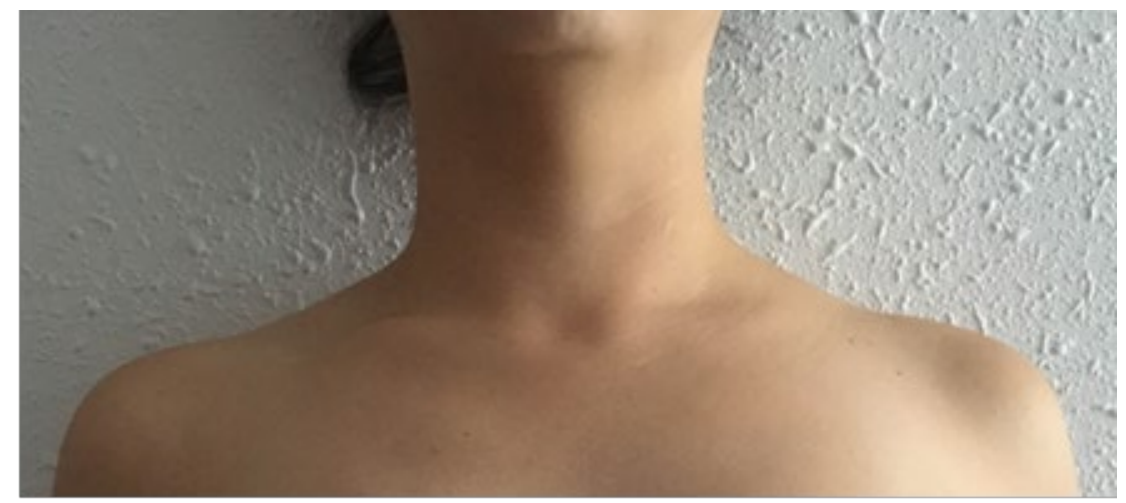

Fig. 9. Vista frontal de la apariencia física después de la consolidación de la fractura.

\section{Discusión}

Tradicionalmente, la mayoría de las fracturas de clavícula se han manejado de manera conservadora debido a las altas tasas de complicaciones del tratamiento quirúrgico (Rowe, 1960). Estudios realizados por Neer (1960) y Rowe (1960) resaltaron la efectividad del tratamiento conservador incluso en fracturas desplazadas, reportando una tasa de no unión menor del 1\%. Algunas revisiones recientes sugieren que la tasa asciente entre $15 \%$ y $20 \%$ (Canadian Orthopaedic Trauma Society, 2007; Hill, McGuire, \& Crosby, 1997; Nowak, Holgersson, \& Larsson, 2005; Zlowodzki et al., 2005) así como consolidación anómala que puede alterar la estructura de la cintura escapular, acortar la distancia hacia el hombro y desalinear las escápulas (Ristevski et al., 2013; Kim, Lee, Jang, Yeom, \& Banks, 2017; Rasyid, 2017). 
Existe controversia acerca de la efectividad del tratamiento conservador en presencia de desplazamiento. Frente a un aparente índice de alteraciones en la consolidación, estudios promueven la fijación quirúrgica primaria en pacientes que presentan deformidad visible o acortamiento de 1,5 o 2cm o más (Van Der Meijden, Gaskill, \& Millett, 2012; Wick, Müller, Kollig, \& Muhr, 2001). Otros abogan por la revisión individualizada de los casos (W. Kim \& McKee, 2008), teniendo en cuenta que en una clavícula muy larga (individuos altos) $2 \mathrm{~cm}$ puede tener menor efecto mecánico que la misma cantidad de acortamiento en una clavícula corta (R. Postacchini, Gumina, Farsetti, \& Postacchini, 2010).

El metaanálisis realizado por Lenza, Buchbinder, Johnston, Belloti, \& Faloppa (2013) destaca que no se encontraron pruebas que indiquen que la intervención quirúrgica tenga beneficios en términos de calidad de vida, y del efecto de la cirugía en el resultado estético debido a la calidad muy baja de las pruebas disponibles en siete ensayos. El tratamiento conservador reduce el riesgo de infección y cirugía secundaria debido a complicaciones, pero no hay certeza de la estimación del efecto y su precisión debido a la baja calidad de la evidencia.

Las fracturas de costillas múltiples son comúnmente tratadas de forma conservadora y a menudo requieren asistencia por medio de ventilación mecánica (Forward, Ollivere, Ng, Coughlin, \& Rollins, 2016). Ante las tasas de complicaciones ha habido un alza en la preferencia de métodos de fijación quirúrgica. Granetzny, Abd El-Aal, Emam, Shalaby, \& Boseila, (2005) identificaron mayores beneficios del tratamiento quirúrgico frente al conservador en el manejo de fracturas de costillas múltiples aisladas y dolorosas y Leinicke, Elmore, Freeman, \& Colditz (2013) concluyeron que la fijación quirúrgica del tórax inestable ofrece mejores resultados que el tratamiento conservador, reduce el porcentaje de mortalidad y el tiempo de asistencia hospitalaria.

En la revisión no se encontraron estudios de politraumatismo tratados de manera conservadora. El caso reportado expone resultados destacables en torno a mejora de la función muscular y respiratoria, tiempo y calidad en la consolidación de las fracturas y disminución del dolor. Se encontraron ventajas asociadas al uso, confort y satisfacción del paciente frente al tratamiento inicial (vendaje en 8 , cabestrillo y corsé elástico). El éxito de la intervención fue la visión integrada de todas las lesiones y sus afectaciones, incluyendo las no óseas. 
Es necesario validar la efectividad del tratamiento, la usabilidad y satisfacción en pacientes con fracturas múltiples en futuros estudios. De ser comprobada su utilidad y conveniencia, este método podría contribuir a minimizar los casos de pseudoartrosis o consolidación anómala y las alteraciones cinemáticas derivadas.

\section{Conclusión}

Se expuso un caso de fracturas múltiples con desplazamiento en paciente con politraumatismo severo tratada con un nuevo método de inmovilización. Los resultados indican que el principio de tracción multidireccional emulado del sistema muscular puede favorecer el proceso de consolidación conjunta de fracturas de clavícula y costillas, brindando excelentes resultados clínicos y físicos.

\section{Referencias}

BARNEA, Y., KASHTAN, H., SKORNICK, Y. y WERBIN, N. (2002). "Isolated rib fractures in elderly patients: mortality and morbidity". Canadian Journal of Surgery. Journal Canadien de Chirurgie, 45(1), 43-46.

CANADIAN ORTHOPAEDIC TRAUMA SOCIETY. (2007). "Nonoperative treatment compared with plate fixation of displaced midshaft clavicular fractures. A multicenter, randomized clinical trial". The Journal of Bone and Joint Surgery. American Volume, 89(1), 1-10.

FORWARD, D. P., OLliVERE, B. J., NG, J. W. G., COUGHLIN, T. A., y ROLLINS, K. E. (2016). "Current concepts in rib fracture fixation". Bone \& Joint $360,5(5), 2-7$.

GRANETZNY, A., ABD EL-AAL, M., EMAM, E., SHALABY, A. y BOSEILA, A. (2005). "Surgical versus conservative treatment of flail chest. Evaluation of the pulmonary status". Interactive CardioVascular and Thoracic Surgery, 4(6), 583587.

HILL, J. M., MCGUIRE, M. H. y CROSBY, L. A. (1997). "Closed treatment of displaced middle-third fractures of the clavicle gives poor results". The Journal of Bone and Joint Surgery, 79(4), 537-539.

KIM, D. S., LEE, D. W., JANG, Y. H., YEOM, J. S. y BANKS, S. A. (2017). "Effects of short malunion of the clavicle on in vivo scapular kinematics". Journal of Shoulder and Elbow Surgery, 26(9), e286-e292.

KIM, W. y MCKEE, M. D. (2008). "Management of Acute Clavicle Fractures". 
Orthopedic Clinics of North America, 39(4), 491-505.

KUO, K.. y KIM, A. M. (2019). Rib Fracture. StatPearls (Vol. 2011). Treasure Island: StatPearls Publishing.

LEINICKE, J. A., ELMORE, L., FREEMAN, B. D. y COLDITZ, G. A. (2013). Operative Management of Rib Fractures in the Setting of Flail Chest". Annals of Surgery, 258(6), 914-921.

LENZA, M., BUCHBINDER, R., JOHNSTON, R. V, BELLOTI, J. C. y FALOPPA, F. (2013). "Surgical versus conservative interventions for treating fractures of the middle third of the clavicle". Cochrane Database of Systematic Reviews, 6(9), CD009363.

NEER, C. S. (1960). "Nonunion of the clavicle". Journal of the American Medical Association, 172, 1006-1011.

NOWAK, J., HOLGERSSON, M. y LARSSON, S. (2005). "Sequelae from clavicular fractures are common: A prospective study of 222 patients". Acta Orthopaedica, 76(4), 496-502.

POSTACCHINI, F., GUMINA, S., DE SANTIS, P. y ALBO, F. (2002). "Epidemiology of clavicle fractures". Journal of Shoulder and Elbow Surgery, 11(5), 452-456.

POSTACCHINI, R., GUMINA, S., FARSETTI, P. y POSTACCHINI, F. (2010). "Long-term results of conservative management of midshaft clavicle fracture". International Orthopaedics, 34(5), 731-736.

RASYID, H. N. (2017). "Influence of Displaced Clavicle Fracture toward Scapular Motion". OALib, 04(05), 1-7.

Ristevski, B., HALl, J. A., PEARCE, D., POTTER, J., FARRUGiA, M. y MCKEE, M. D. (2013). "The radiographic quantification of scapular malalignment after malunion of displaced clavicular shaft fractures". Journal of Shoulder and Elbow Surgery, 22(2), 240-246.

ROBINSON, C. M. (1998). "Fractures of the clavicle in the adult. Epidemiology and classification". The Journal of Bone and Joint Surgery. British Volume, 80(3), 476484.

ROWE, C. R. (1960). "An atlas of anatomy and treatment of midclavicular fractures". Clinical Orthopaedics and Related Research, 58(10), 29-42.

TODD, S. R., MCNALLY, M. M., HOLCOMB, J. B., KOZAR, R. A., KAO, L. S., GONZALEZ, E. A. y MOORE, F. A. (2006). "A multidisciplinary clinical pathway decreases rib fracture-associated infectious morbidity and mortality in high-risk trauma patients". American Journal of Surgery. 
VAN DER MEIJDEN, O. A., GASKILL, T. R., y MILLETT, P. J. (2012). "Treatment of clavicle fractures: current concepts review". Journal of Shoulder and Elbow Surgery, 21(3), 423-429.

WICK, M., MÜLLER, E. J., KOLLIG, E., y MUHR, G. (2001). "Midshaft fractures of the clavicle with a shortening of more than $2 \mathrm{~cm}$ predispose to nonunion". Archives of Orthopaedic and Trauma Surgery, 121(4), 207-211.

ZLOWODZKI, M., ZELLE, B. A., COLE, P. A., JERAY, K., MCKEE, M. D., y EVIDENCE-BASED ORTHOPAEDIC TRAUMA WORKING GROUP. (2005). "Treatment of acute midshaft clavicle fractures: systematic review of 2144 fractures: on behalf of the Evidence-Based Orthopaedic Trauma Working Group". Journal of Orthopaedic Trauma, 19(7), 504-507.

\section{Imágenes}

MÚSCULOS.ORG (n,d). Músculo Deltoides. [imagen] Disponible en: https://www.musculos.org/musculo-deltoides.html [Acceso 2 Mayo de 2019].

MÚSCULOS.ORG (n,d). Músculo Dorsal Ancho. [imagen] Disponible en: https://www.musculos.org/musculo-dorsal-ancho.html [Acceso 2 Mayo de 2019].

MÚSCULOS.ORG (n,d). Músculo Oblicuo Mayor del Abdomen. [imagen] Disponible en: https://www.musculos.org/musculo-abdominales-oblicuo-mayorabdomen.html [Acceso 2 Mayo de 2019].

MÚSCULOS.ORG (n,d). Músculo Pectoral Mayor [imagen] Disponible en: https://www.musculos.org/musculo-pectoral-mayor.html [Acceso 2 Mayo de 2019].

MÚSCULOS.ORG (n,d). Músculo Serrato Mayor [imagen] Disponible en: https://www.musculos.org/musculo-serrato-mayor.html [Acceso 2 Mayo de 2019].

MÚSCULOS.ORG (n,d). Músculo Trapecio. [imagen] Disponible en: https://www.musculos.org/musculo-trapecio.html [Acceso 2 Mayo de 2019].

OPENSTAX CNX (2013). Cintura escapular. [imagen] Disponible en: https://cnx.org/resources/57d6e706ac983c0aa78022708939083d37027c4a/Figure 38 01_10.jpg [Acceso 8 Mayo de 2019]. CC 4.0 


\title{
Análisis biomecánico para confirmar el diagnóstico en neurorrehabilitación
}

\author{
de los Reyes-Guzmán, Ana ${ }^{a}$; López-Dolado, Elisab ${ }^{b}$ Pérez-Rizo, Enrique ${ }^{a}$; Lozano- \\ Berrio, Vicente ${ }^{a}$; Gil-Agudo, Ángel ${ }^{\mathrm{b}}$ y del-Ama, Antonio J. ${ }^{a}$ \\ aUnidad de Biomecánica y Ayudas Técnicas, Hospital Nacional de Parapléjicos (SESCAM), Toledo, \\ España,\{adlos;enriquep;vlozanob;ajdela\}@sescam.jccm.es \\ bServicio de Rehabilitación, Hospital Nacional de Parapléjicos (SESCAM), Toledo, España, \{elopez, \\ amgila\}@sescam.jccm.es
}

\begin{abstract}
In cervical spinal cord injured (SCI) patients, upper limbs strength is affected to a greater or lesser extent, producing dependence on the execution of ADLs. If the lesion is incomplete, the gait ability can be preserved. In this context, it is difficult to make a correct clinical diagnosis and the photogrammetry equipments constitute a tool of great value to determine with objectivity motor sequelae. The objective is to present the biomechanics methodology of upper and lower limbs applied to a case study. It is a 61year-old male patient who suffered an incomplete SCI of traumatic etiology that had previously suffered a traumatic brain injury. Through photogrammetry, the range of motion of the hip, knee and ankle joints were analyzed during the cycles of the gait and the shoulder, elbow and wrist joints, as well as a series of kinematic indices describing the ability and dexterity of the upper limb movement.

A very symmetrical gait pattern was obtained in both lower limbs. However, overall functionality and skill indices in both upper limbs present a high asymmetry between them. Biomechanical tools demonstrate aspects of motor control not easily visible with clinical tests and perfect diagnosis of complex cases.
\end{abstract}

Keywords:biomechanics, kinematics, kinetic, lower limb, upper limb, stroke, spinal cord injury.

\section{Resumen}

En los pacientes con lesión medular cervical (LMC) se ve comprometida en mayor o menor medida la fuerza de las extremidades superiores, lo que se traduce en dependencia para las AVDs. Si la lesión es incompleta, puede preservarse la capacidad de marcha. En este contexto, resulta dificil realizar un diagnóstico clínico correcto y los equipos de fotogrametría constituyen 
una herramienta de gran valor para objetivar las secuelas motoras. El objetivo es presentar la metodología biomecánica de miembros superiores e inferiores aplicada a un caso de estudio. Se trata de un paciente varón de 61 años que padeció una LMC incompleta de etiología traumática que, previamente, había sufrido un TCE. Mediante fotogrametría se analizaron los recorridos articulares de la cadera, rodilla y tobillo durante los ciclos de la marcha y del hombro, codo y muñeca, así como una serie de índices cinemáticos descriptores del movimiento del miembro superior.

Se obtuvo un patrón de marcha muy simétrico en ambos miembros inferiores. Sin embargo, la funcionalidad global y los indices de destreza en ambos miembros superiores presentaron una marcada asimetría entre ellos. Las herramientas biomecánicas evidencian aspectos del control motor no fácilmente visibles con los tests clínicos y que perfeccionan el diagnóstico de los casos complejos.

Palabras clave:biomecánica, cinemática, cinética, miembro inferior, miembro superior, ACV, Lesión medular.

\section{Introducción}

En los últimos años, el aumento de la esperanza de vida ha traído como consecuencia un incremento en el número de traumatismos del sistema nervioso central (SNC) en la población de más edad. A su vez, las secuelas neurológicas aumentan el riesgo de caídas y, por lo tanto, el riesgo de sufrir nuevos eventos traumáticos.

La afectación de la extremidad superior es una de las secuelas más habituales en las lesiones del SNC (Parker, 1986) y, en el caso de la lesión medular afecta a más del 50\% de los casos (Wyndaele, 1996).

En los pacientes con lesión medular cervical (LMC) se ve comprometida en mayor o menor medida la fuerza de las extremidades superiores, lo que se traduce en dependencia para las AVDs y pérdida de autonomía personal. Si la lesión es incompleta, puede preservarse la capacidad de marcha. Cuando hay secuelas sensitivomotoras previas, éstas ocultan los síntomas y signos de localización de la lesión medular y resulta difícil realizar un diagnóstico clínico correcto. Por este motivo, resulta necesaria la aplicación de elementos objetivos de medida y sistemas precisos de análisis de movimiento para describir con una mayor precisión y especificidad el movimiento humano.

En este contexto, los estudios biomecánicos son una herramienta de gran valor para objetivar la marcha humana y la ejecución de movimientos correspondientes a actividades 
funcionales de los miembros superiores. Aplicados al análisis de la marcha humana, su utilidad se centra, fundamentalmente, en servir de herramienta de valoración en pacientes que presentan un cuadro conocido para tomar decisiones clínicas y planificar su tratamiento, así como para evaluar posteriormente la eficacia de las medidas adoptadas. (Gil-Agudo, 2019).

En cuanto a los estudios para objetivar la función del miembro superior son numerosos los realizados en población sana (Namdari, 2012; Murgia, 2010; Aizawa, 2010; van Andel, 2008; Petuskey, 2007; Murphy, 2006; Magermans, 2005). Posteriormente, estos estudios se fueron extendiendo para cuantificar objetivamente la capacidad funcional y rangos de movimiento articular en presencia de patologías neurológicas. En cuanto a estas enfermedades, las estudiadas con más frecuencia han sido el accidente cerebrovascular (ACV) (Kim, 2014; Murphy, 2013; Murphy, 2011; Osu, 2011; Lang, 2006) y la Parálisis Cerebral (Klotz, 2013; Butler, 2010). Todavía los estudios aplicados a la LM son escasos frente a los realizados en otras patologías. No obstante, existe un trabajo previo que revisa los estudios cinemáticos del miembro superior realizados en pacientes con LM (Mateo, 2015).

Actualmente, en nuestro grupo, ambas pruebas biomecánicas están bien consolidadas y establecidas para su aplicación en pacientes con Lesión Medular o cualquier otra patología neurológica que entre sus secuelas produce trastornos de la marcha humana y afectación de la función de los miembros superiores. Sin embargo, no se tiene evidencia de estudios previos que apliquen ambas pruebas biomecánicas conjuntamente a la muestra analizada con el objetivo de ayudar al diagnóstico clínico. Así, el objetivo de este trabajo es presentar la metodología biomecánica de miembros superiores e inferiores aplicada a un caso de estudio.

\section{Métodos}

Se trata de un estudio descriptivo, experimental de estudio de un caso, el de un varón de 61 años que sufre una lesión medular cervical traumática aguda incompleta al caerse de una bicicleta. El paciente había sufrido cinco años antes un traumatismo craneoencefálico con resultado de hemorragia intraparenquimatosa parietal izda que le había dejado como secuela una monoparesia espástica derecha mínima. Las pruebas de imagen tras la caída evidenciaron una fractura de la lámina izda C6 sin desplazamiento vertebral ni estenosis de canal con contusión medular $\mathrm{C} 4$ y afectación de la musculatura cervical posterior y los ligamentos interespinosos desde $\mathrm{C} 2-\mathrm{C} 5$. Se desestima tratamiento quirúrgico y se le remite a un hospital monográfico de lesión medular para que realice un programa de rehabilitación integral. A su llegada a dicho centro presentaba una lesión medular de nivel C4 incompleto sensitivomotor ASIA D que no se ajustaba bien a ninguno de los síndromes clínicos de 
lesión incompleta, lo que dificultaba establecer el pronóstico funcional, pues tanto las excalas clínicas como los estudios habituales de neuroimagen y neurofisiológicos estaban interferidos por las secuelas sobre el miembro superior derecho del traumatismo craneoencefálico antiguo. Se decidió analizar el movimiento de los miembros superiores y la marcha del paciente con herramientas biomecánicas, con el objetivo de obtener datos objetivos y cuantitativos del control motor en este paciente que pudieran enriquecer el diagnóstico clínico.

Ambas pruebas biomecánicas se realizaron en el período subagudo aunque no simultáneamente. Se realizaron instrumentando al paciente con el equipo de fotogrametría Codamotion (Charnwood Dynamics, Ltd, UK) basado en marcadores activos, muestreando a una frecuencia de $200 \mathrm{~Hz}$. Adicionalmente, para el análisis de la marcha se utilizaron dos plataformas dinamométricas Kistler para registrar de forma simultánea la fuerza de reacción durante la fase de apoyo en ambos miembros inferiores.

\subsection{Análisis biomecánico de la marcha}

Para la realización del estudio biomecánico de la marcha se posicionaron 22 marcadores activos los miembros inferiores y en la pelvis, siguiendo el modelo descrito previamente por otros autores (Kadaba, 1989). Los marcadores se posicionaron en las marcas anatómicas descritas en nuestro trabajo previo (Gil-Agudo, 2009) para analizar los recorridos articulares de la cadera, rodilla y tobillo durante los ciclos de la marcha. A partir de las posiciones de los marcadores se calcularon los desplazamientos de las siguientes referencias anatómicas: espinas ilíacas anterosuperiores, espinas ilíacas posterosuperiores, epicondilos medial y lateral, ambos maléolos, calcáneo y $5^{\mathrm{a}}$ articulación metacarpofalágica.

Una vez instrumentado el paciente, éste se situó al inicio del pasillo de la marcha. Antes de iniciar la adquisición de pruebas el sujeto realizó varias pasadas por el pasillo de macha a modo de adaptación, con su velocidad de marcha habitual. Posteriormente, se registraron una serie de pasadas hasta lograr un mínimo de cinco pruebas correctas (en función del pisado completo sobre las plataformas).

\subsubsection{Procesamiento de los datos y variables analizadas}

Una vez terminada la adquisición, se procede al análisis de los resultados mediante la utilización de un modelo biomecánico de ambos miembros inferiores escalado a las dimensiones antropométricas del paciente (Fig.1A).

En cuanto a la cinemática de los miembros inferiores se analizaron los valores máximos y mínimos tanto de la fase de apoyo como de la de balanceo, el momento del ciclo en que 
aparecen (expresado en \% del ciclo de marcha), la amplitud articular (diferencia entre el valor máximo y el mínimo de todo el ciclo independientemente de si se sitúa en el apoyo o en el balanceo) así como, los siguientes valores críticos: contacto inicial y despegue de dedos. La recogida de datos se efectuó de todas las articulaciones, cadera, rodilla y tobillo, en cada uno de sus recorridos articulares posibles.

Asimismo, se analizaron los parámetros espaciotemporales de la marcha como la velocidad de marcha $(\mathrm{m} / \mathrm{s})$, duración del ciclo de marcha $(\mathrm{s})$, longitud del ciclo $(\mathrm{cm})$, duración del paso (s), cadencia (pasos/min), duración de la fase de apoyo y de oscilación (expresado en $\%$ de la duración del ciclo de marcha).

\subsection{Análisis cinemático del miembro superior}

Los estudios cinemáticos del miembro superior se realizan simulando la AVD de beber de un vaso. El setup experimental y la configuración del laboratorio se describe en detalle en un trabajo previo nuestro realizado en población sana y en pacientes con LMC (de los Reyes-Guzmán, 2010). En ese estudio, todos los participantes eran diestros y realizaron el gesto de beber con el miembro superior derecho a velocidad libre.

Sin embargo, en este trabajo se realiza el estudio cinemático en ambos miembros superiores aunque no de forma simultánea, debido a la complejidad del estudio en cuanto a instrumentación. Por tanto, se realizan en sesiones diferentes, muy próximas entre sí para asegurar que no se producen cambios en la capacidad funcional del paciente.

\subsubsection{Sesiones experimentales}

Las sesiones experimentales sobre cada miembro superior se realizaron una sola vez. El registro del movimiento se realizó con el mismo equipo de fotogrametría que el utilizado para el análisis de la marcha humana.

En cada sesión se instrumentó el tronco y brazo cuyo movimiento se analiza con 21 marcadores de fotogrametría: 8 se ubicaron en prominencias óseas para formar el modelo biomecánico y los otros 13 se localizaron en clusters de marcadores para conocer la posición y orientación de los segmentos corporales durante el movimiento. Estos 13 marcadores se reparten de la siguiente forma: 9 repartidos en clusters de 3 marcadores ubicados en tronco, brazo y antebrazo; 3 marcadores en la parte dorsal de la mano sobre la piel y uno adicional en el tronco.

Una vez instrumentado el paciente, la actividad analizada consistía en alcanzar y agarrar el vaso desde la posición de inicio, llevar el vaso a la boca, beber (equivalente a un trago), llevar el vaso al área marcada en la mesa y volver a la posición de inicio. Antes de registrar 
el movimiento todos los sujetos realizaron la actividad dos veces a modo de aclimatación. Se capturaron y analizaron 5 registros válidos.

\subsubsection{Procesamiento de los datos y variables analizadas}

Para el procesamiento de los datos se utilizó el software Visual3D (C-Motion, Inc, USA) en el que se implementó un programa de tratamiento y procesamiento de la señal encargado de obtener las señales del movimiento de las distintas articulaciones involucradas (hombro, codo y muñeca). La frecuencia de muestreo era de $200 \mathrm{~Hz}$. Se analizaron cinco registros y la media de las cinco medidas fue el valor final para cada una de las variables analizadas.

Para el análisis cinemático del miembro superior derecho, se implementó en Visual3D un modelo biomecánico de 9 grados de libertad: 2 en el tronco, 3 en el hombro, 2 en el codo y 2 en el carpo. Para cada segmento del modelo se definió un sistema de coordenadas local siguiendo las recomendaciones de la Sociedad Internacional de Biomecánica (ISB) (Wu, 2005) (Fig.1B). De la mismaforma se implementó el modelo para analizar el movimiento del miembro superior izquierdo.
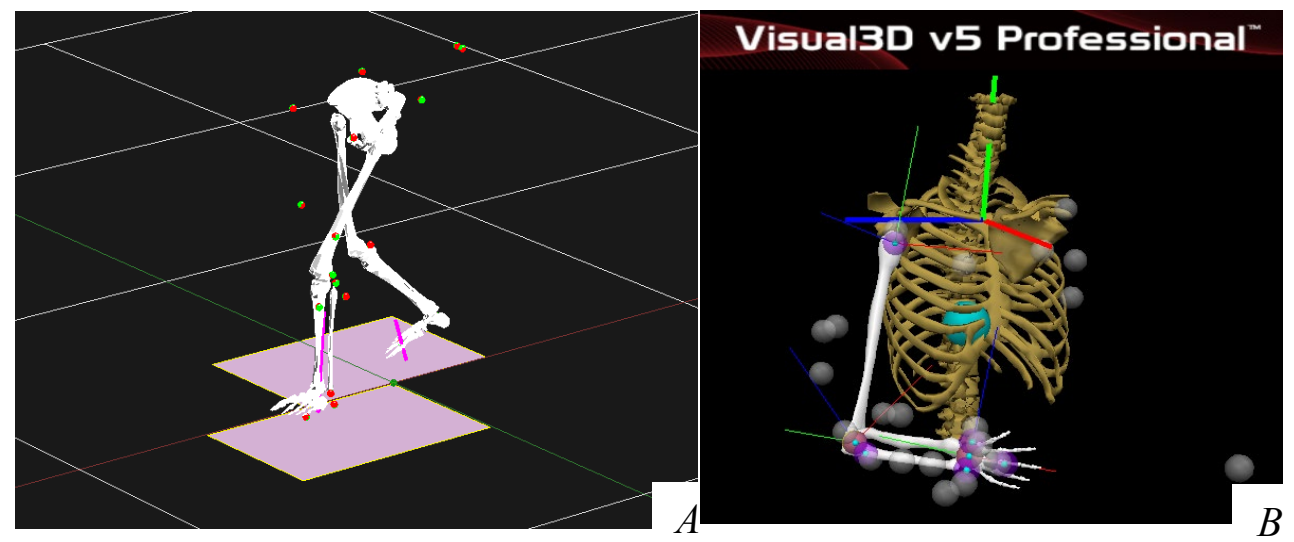

Fig.1: A) Modelo biomecánico de los miembros inferiores escalado a las dimensiones antropométricas del paciente (2016). B) Modelo biomecánico del miembro superior derecho escalado a las dimensiones antropométricas del paciente (2016).

En cada uno de los registros se identificó un ciclo completo de la actividad de beber. Se consideró que el inicio del ciclo se correspondía con el inicio del desplazamiento del marcador situado en la cabeza del tercer metacarpiano y el final de dicho ciclo cuando ese marcador se volvía a situar en la posición inicial tras realizar la actividad. Basándonos en estudios previos (Murphy, 2006; de los Reyes-Guzmán, 2010), se establecieron una serie de fases para facilitar el análisis de la actividad: alcance (incluye coger el vaso), transporte proximal, beber, transporte distal (incluye soltar el vaso) y retorno a inicio. 
Una vez recogidos y analizados los registros, a partir de los recorridos articulares y variables como la posición y velocidad de la mano, medida a partir del marcador en la cabeza del tercer metacarpiano, se computaron una serie de índices cinemáticos descriptores del movimiento, relacionados con destreza y habilidad global del miembro superior, como son precisión, agilidad, eficiencia, suavidad y coordinación (de los ReyesGuzmán, 2017; de los Reyes-Guzmán, 2016).

\section{Resultados}

A continuación se muestran la evolución neurológica y funcional del paciente y los resultados obtenidos en ambas pruebas biomecánicas realizadas.

\subsection{Evolución neurológica}

A su ingreso, el paciente presentaba una lesión medular cervical de nivel C4 incompleta sensitivomotora grado D según la escala ASIA (Kirshblum, 2011) (Fig 2A y 2B) y al alta a los seis meses de evolución, una vez concluido el programa rehabilitador intensivo, mostraba una clara mejoría global, tanto sensitiva como motora y funcional (Fig. 2 C, D y E respectivamente).
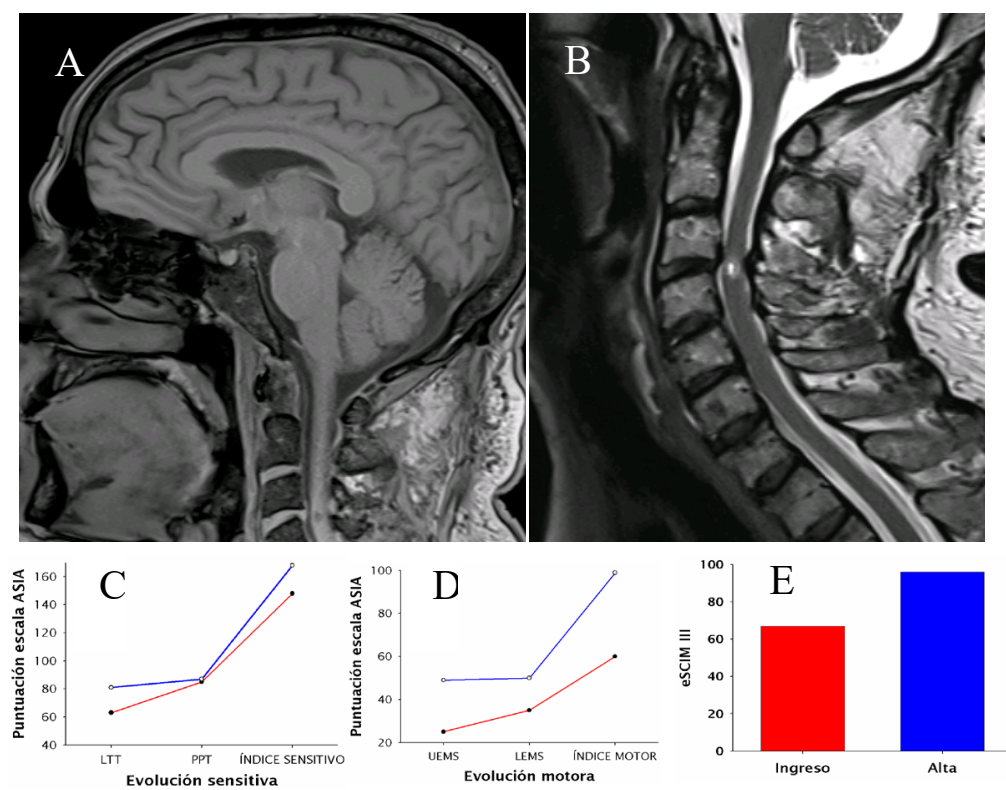

Fig.2: A) Corte sagital en T1 de resonancia magnética craneal a los 6 meses de lesión, donde se comprueba la normalidad del encéfalo y la lesión medular en C4. B) Corte sagital en T2 de resonancia magnética de columna cervical. $\boldsymbol{C}, \boldsymbol{D} \boldsymbol{y} \boldsymbol{E})$ Evolución de la lesión medular desde un punto de vista sensitivo, motor y funcional, respectivamente. 


\subsection{Análisis biomecánico de la marcha}

Parámetros temporales: Se detectaron las habituales alteraciones en la marcha de personas con lesión medular, es decir, disminución de la velocidad, de las longitudes de ciclo y de paso, de la frecuencia de ciclos/minuto y de la cadencia. Por el contrario, aumento de la duración de ciclo y de paso y el porcentaje de duración de la fase de apoyo (tabla 2).

Cinemática articular: En el plano transversal de la pelvis existía una rotación pélvica con elevación de la hemipelvis derecha. En el plano frontal, se observaba la cadera derecha en abducción durante la fase de apoyo, probablemente para aumentar la base de apoyo y ganar estabilidad en el apoyo monopodal. La cadera derecha se mantenía en rotación externa a lo largo del ciclo. El pico normal de flexión de rodilla en la fase de oscilación de la marcha estaba disminuido en ambas rodillas, que se posicionaban en rotación externa siempre en el plano transversal. En el tobillo, se observaba que el pico normal de flexión plantar en el despegue de dedos, necesario para el adecuado despegue del pie y avance del miembro inferior durante la fase de oscilación, estaba disminuido. Por último, ambos pies se mantenían en abducción durante el ciclo de la marcha (tabla 3).

Tabla 1. Resultados de los parámetros espaciotemorales de ambas extremedades inferiores del paciente y de la base de datos de normalidad.

\begin{tabular}{ccccc}
\hline $\begin{array}{c}\text { Parámetros } \\
\text { espacio-temporales }\end{array}$ & Izquierdo & Derecho & Normalidad & $\begin{array}{c}\text { Desviación } \\
\text { estándar }\end{array}$ \\
\hline Velocidad (m/s) & $0.77 \pm 0.03$ & $0.7 \pm 0.03$ & 1.27 & 0.12 \\
Longitud ciclo (m) & $1 \pm 0.03$ & $0.95 \pm 0.02$ & 1.33 & 0.08 \\
Duración Ciclo (s) & $1.31 \pm 0.03$ & $1.35 \pm 0.04$ & 1.05 & 0.08 \\
Ciclos / Minuto & $45.9 \pm 1.08$ & $44.58 \pm 1.25$ & 57.33 & 4.16 \\
Longitud paso (m) & $0.52 \pm 0.01$ & $0.49 \pm 0.03$ & 0.67 & 0.04 \\
Duración paso (s) & $0.65 \pm 0.02$ & $0.67 \pm 0.02$ & 0.53 & 0.04 \\
Pasos / Minuto & $92.9 \pm 2.91$ & $90.4 \pm 3.23$ & 114.65 & 8.30 \\
Porcentaje & $64.95 \pm 1.38$ & $67.45 \pm 0.96$ & 63.13 & 1.62 \\
apoyo(\%) & & $0.46 \pm 0.02$ & 0.39 & 0.03 \\
Apoyo unipodal (s) & $0.44 \pm 0.02$ & $0 \pm 0$ & 0.14 & 0.02 \\
Apoyo bipodal (s) & $0.22 \pm 0.01$ & & & \\
Contacto inicial de & $50.65 \pm 1.2$ & $50.75 \pm 1.11$ & & \\
pie contralateral (\%) & & & & \\
\hline
\end{tabular}


de los Reyes-Guzmán, A.; López-Dolado, E.; Pérez-Rizo, E.; Lozano-Berrio, V.; Gil-Agudo, A. y del-

Tabla 2. Resultados de las variables cinemáticas articulares (expresadas en ${ }^{0}$ ) del paciente y de la base de datos de normalidad.

\begin{tabular}{|c|c|c|c|c|}
\hline $\begin{array}{c}\text { Ángulos } \\
\text { articulares plano } \\
\text { sagital }\left(^{\circ}\right)\end{array}$ & Izquierdo. & Derecho & Normalidad & $\begin{array}{c}\text { Desviación } \\
\text { estándar }\end{array}$ \\
\hline Rango de cadera & $35.93 \pm 0.3$ & $32.35 \pm 1.42$ & 42.30 & 4.16 \\
\hline Máximo cadera & $\begin{array}{c}37.83 \pm \\
37.83\end{array}$ & $35.57 \pm 0.41$ & 39.92 & 7.26 \\
\hline Mínimo cadera & $1.9 \pm 0.43$ & $3.22 \pm 1.18$ & -0.39 & 7.26 \\
\hline Rango de rodilla & $53.57 \pm 1.7$ & $47.05 \pm 1.14$ & 61.84 & 3.46 \\
\hline Máximo rodilla & $53.17 \pm 1.22$ & $49.33 \pm 1.87$ & 63.03 & 3.50 \\
\hline Mínimo rodilla & $-0.4 \pm 0.83$ & $2.28 \pm 1.02$ & 1.87 & 3.81 \\
\hline Rango de tobillo & $17.02 \pm 1.95$ & $16.9 \pm 0.84$ & 31.57 & 5.42 \\
\hline Máximo tobillo & $7.64 \pm 1.56$ & $9.14 \pm 1.1$ & 12.40 & 2.47 \\
\hline Mínimo tobillo & $-9.38 \pm 0.64$ & $-7.76 \pm 0.73$ & -17.21 & 4.81 \\
\hline Rango de cadera & $35.93 \pm 0.3$ & $32.35 \pm 1.42$ & 42.30 & 4.16 \\
\hline
\end{tabular}

\subsection{Análisis cinemático del miembro superior}

Los resultados relativos al estudio biomecánico del miembro superior derecho e izquierdo se representan en la tabla 3 , diferenciados según se trate de rangos de movimiento articular, variables temporales e índices cinemáticos descriptores del movimiento.

\subsubsection{Rangos de movimiento articular (ROM)}

En el análisis del miembro superior derecho se obtuvo un ROM total de $82^{\circ}$ en el hombro, siendo $42.83^{\circ}$ en el movimiento de flexión-extensión, $25.14^{\circ}$ en el movimiento de abducción-adducción y $14.03^{\circ}$ en rotación externa-interna; en la articulación del codo se obtuvo un total de $131.15^{\circ}$ de los que $90.46^{\circ}$ corresponden al movimiento de flexiónextensión y 40.69 a la pronación-supinación del antebrazo; en cuanto a la muñeca se midieron $14.19^{\circ}$ en el movimiento de flexión-extensión. 
Tabla 3. Resultados de las variables cinemáticas en ambas evaluaciones realizadas al paciente

\begin{tabular}{ccc}
\hline $\begin{array}{c}\text { Variables } \\
\text { cinemáticas }\end{array}$ & $\begin{array}{c}\text { Miembro superior } \\
\text { derecho }\end{array}$ & $\begin{array}{c}\text { Miembro superior } \\
\text { izquierdo }\end{array}$ \\
\hline ROM ( ${ }^{\text {) }}$ & & \\
Flex-ext hombro & 42.83 & 36.08 \\
Abd-add hombro & 25.14 & 15.85 \\
Rot. Ext-Int & 14.03 & 19.53 \\
hombro & & 74.57 \\
Flex-ext codo & 90.46 & 45.02 \\
Pron-sup codo & 40.69 & 76.25 \\
Flex-ext muñeca & 14.19 & 8.23 \\
Tiempo (s) & 8.10 & 0.76 \\
Vel. max (m/s) & 0.70 & 0.24 \\
Vel. med (m/s) & 0.23 & 104.78 \\
Índices \\
cinemáticos (\%)
\end{tabular}

En cuanto al miembro superior izquierdo se obtuvo un ROM total de $71.46^{\circ}$ en el hombro, siendo $36.08^{\circ}$ en el movimiento de flexión-extensión, $15.85^{\circ}$ en el movimiento de abducción-adducción y $19.53^{\circ}$ en rotación externa-interna; en la articulación del codo se obtuvo un total de $119.59^{\circ}$ de los que $74.57^{\circ}$ corresponden al movimiento de flexiónextensión y 45.02 a la pronación-supinación del antebrazo; en cuanto a la muñeca se midieron $76.25^{\circ}$ en el movimiento de flexión-extensión. 


\subsubsection{Variables temporales}

En cuanto a la duración del ciclo completo de movimiento, los resultados fueron muy parecidos en ambos miembros superiores: $8.10 \mathrm{~s}$ en el caso del miembro superior derecho y $8.23 \mathrm{~s}$ en el miembro superior izquierdo. Lo mismo ocurrió en cuanto a las velocidades de movimiento (Tabla 3).

\subsection{3. Índices cinemáticos}

Los resultados de los índices cinemáticos se representan en \%, relativos al patrón de referencia formado por un grupo de personas sanas (de los Reyes-Guzmán, 2016; de los Reyes Guzmán, 2017). Se ha obtenido en el miembro superior izquierdo un menor desempeño en los índices Precisión (64.92) y Agilidad (62.79) que aquellos en el miembro superior derecho (91.68 y 88.90, respectivamente). Sin embargo en el izquierdo se ha obtenido un mejor desempeño en cuanto a los índices suavidad y coordinación, que superan la puntuación del patrón de referencia. Estos resultados se pueden observar de forma más sencilla en la Fig.3.

\section{ÍNDICES DE VALORACIÓN FUNCIONAL}

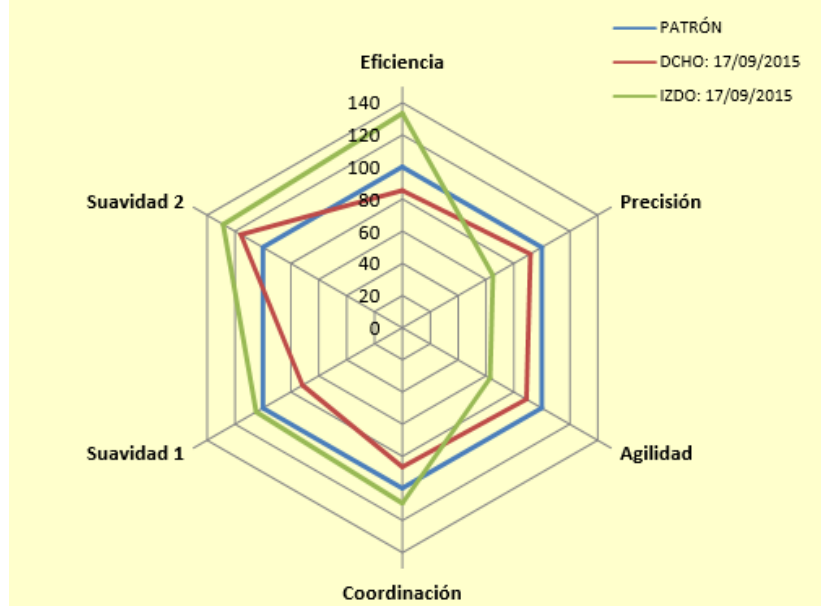

Fig. 3. Representación gráfica de los índices cinemáticos relativos a las evaluaciones del miembro superior derecho (rojo) e izquierdo (verde) (2015).

\section{Discusión}

El paciente que se presenta había sufrido dos lesiones traumáticas del sistema nervioso: un primer traumatismo craneoencefálico que le dejó como secuela una monoparesia espástica 
derecha cinco años atrás y una lesión medular cervical incompleta aguda. Su edad y el mecanismo de lesión apuntaban hacia el síndrome centromedular o de Schneider (Schneider, 1958). Sin embargo, la neuroimagen inicial mostraba un gran componente edematoso y no se ajustaba a la localización centromedular y el paciente había experimentado una clara progresión hacia la mejoría neurológica global, pero ésta había sido mayor en los miembros superiores que en los inferiores (Fig. 2C y 2D), justo al contrario de lo esperado en el síndrome de Schneider. Además, a pesar de que la exploración clínica de la fuerza voluntaria residual de los brazos era bastante simétrica, el paciente refería grandes diferencias en la ejecución motora entre el brazo derecho y el izquierdo.

Dado que la práctica totalidad de la recuperación funcional en los miembros superiores tras una lesión medular cervical sucede en los seis primeros meses en el síndrome centromedular (McKinley, 2007), se decidió evaluar la calidad de la función motriz mediante estudios biomecánicos, que mostraron: 1) que la función locomotriz de ambas piernas era simétrica y se encontraba más próxima a la normalidad que la de los brazos; 2) que ambos miembros superiores estaban severamente afectados pero de manera asimétrica como refería el paciente; 3) que las diferencias entre los miembros superiores se debían la coincidencia en el brazo derecho de las secuelas del traumatismo craneoencefálico antiguo y de la lesión medular cervical actual y se expresaban y cuantificaban consistentemente gracias a los índices cinemáticos. Se concluyó entonces que el diagnóstico más probable del paciente era un síndrome centromedular C4 ASIA D y que la función de ambos brazos, en consecuencia, tenía un pronóstico de recuperación funcional en adelante muy restringido. La resonancia magnética de control realizada a los 6 meses mostró la mielopatía centromedular (Fig. 2A y 2B) y las exploraciones sucesivas al año y a los dos años evidenciaron estancamiento de la manipulación con mejoría discreta en la marcha.

Una limitación del presente trabajo es que la realización de ambos estudios biomecánicos fue secuencial y no simultánea, si bien entre la realización del estudio de marcha y el de los miembros superiores se realizaron en la misma semana. Existe muy pocos trabajos cuya metodología utilice simultáneamente el análisis biomecánico de los miembros superiores y de la marcha. Sin duda, la instrumentación necesaria para aplicar esta doble metodología requiere largos tiempos de intrumentación y probablemente la interpretación de los resultados obtenidos no sea sencilla. Sin embargo, el presente trabajo sugiere que contar con ella podría ser de gran ayuda para mejorar la potencia diagnóstica y para entender mejor la motricidad residual global del paciente que sufre una lesión neurológica compleja. 


\section{Conclusión}

El presente trabajo pretende poner de relieve la capacidad de los estudios biomecánicos para exclarecer dudas diagnósticas o pronósticas de la práctica clínica diaria en neurorrehabilitación gracias a que aportan datos objetivos y cuantitativos del movimiento.

Metodológicamente, se trata de estudios que poseen una elevada complejidad en cuanto a la instrumentación necesaria en el paciente y al procedimiento de análisis de los datos obtenidos. Aun así, hoy en día, siguen siendo de especial interés por tratarse de la tecnología gold-standard en análisis de movimiento dentro del entorno clínico.

\section{Referencias}

AIZAWA, J., MASUDA, T., KOYAMA, T., KOJIISOKZAKI, K.N., OKAWA, A., MORITA, S., (2010). "Three dimensional motion of the upper extremity joints during various activities of daily living”. J Biomech, vol. 43, pp. 2915-2922.

BUTLER, E.E., LADD, A.L., LOUIE, S.A., LAMONT, L.E., WONG, W., ROSE, J., (2010). "Temporalspatial parameters of the upper limb during a reach \& grasp cycle for children". Gait Posture, vol. 32, pp. 301-306.

DE LOS REYES-GUZMÁN, A., GIL-AGUDO, A., PEÑASCO-MARTÍN, B., SOLÍS-MOZOS, M., DEL AMA-ESPINOSA, A., y PÉREZ-RIZO, E. (2010). "Kinematic analysis of the daily activity of drinking from a glass in a population with cervical spinal cord injury". Journal of neuroengineering and rehabilitation, 7(1), 41.

DE LOS REYES-GUZMÁN, A., DIMBWADYO-TERRER, I., PÉREZ-NOMBELA, S., MONASTERIO-HUELIN, F., TORRICELLI, D., PONS, J.L., y GIL-AGUDO, A. (2016). "Novel kinematic indices for quantifying movement agility and smoothness after cervical Spinal Cord Injury". NeuroRehabilitation, 38(2), 199-209.

DE LOS REYES-GUZMÁN, A., DIMBWADYO-TERRER, I., PÉREZ-NOMBELA, S., MONASTERIO-HUELIN, F., TORRICELLI, D., PONS, J.L., y GIL-AGUDO, A. (2017). "Novel kinematic indices for quantifying upper limb ability and dexterity after cervical spinal cord injury". Medical \& biological engineering \& computing, 55(5), 833-844.

GIL-AGUdO, A., PÉREZ-RIZO, E., DEL AMA-ESPINOSA, A., CRESPO-RUIZ, B., PÉREZNOMBELA, S., Sánchez-Ramos A., (2009). "Comparative biomechanical gait analysis of patients with central cord syndrome walking with one and two crutches". Clin Biomech (Bristol Avon) 24:551557.

GIL-AGUDO (2019). "Protocolo de valoración biomecánica de las alteraciones de la marcha". Medicine, 12(75):4462-6.

KADABA, M.P., RAMAKRISHNAN, H.K., WOOTTEN, M.E., GAINEY, J., GORTON, G., COCHRAN, G.V. (1989). "Repeatability of kinematic, kinetic, and electromyographic data in normal adult gait". J Orthop Res; 7(6): 849-860. 
KIM, K., SONG, W.K., LEE, J., LEE, H.Y., PARK, D.S., KO, B.W., KIM, J., (2014). "Kinematic analysis of upper extremity movement during drinking in hemiplegic subjects", Clin Biomech, doi: 10.1016/j.clinbiomech.2013.12.013.

KIRSHBLUM S.C., BURNS S.P., BIERING-SORENSEN F., DONOVAN W., GRAVES D.E., JHA A., JOHANSEN M., JONES L., KRASSIOUKOV A., MULCAHEY MJ, SCHMIDT-READ M., WARING W. (2011). "International standards for neurological classification of spinal cord injury (Revised 2011)". Journal of Spinal Cord Medicine, 34 (6): 535-46.

KLOTZ, M.C.M., KOST, L., BRAATZ, F., EWERBECK, V., HEITZMANN, D., GANTZ, S., DREHER, T., WOLF, S.I., (2013). "Motion capture of the upper extremity during activities of daily living in patients with spastic hemiplegic cerebral palsy". Gait Posture, 38,pp. 148-152.

LANG, C.E., WAGNER, J.M., EDWARDS, D.F., SAHMANN, S.A., DROMERICK, A.W., (2006). "Recovery of grasp versus reach in people with hemiparesis poststroke".Neurorehabil Neural Repair, vol. 20, pp. 444-454.

MAGERMANS, D., CHADWICK, E., VEEGER, H., VAN DER HELM, F., (2005). "Requirements for upper extremity motions during activities of daily living". Clin Biomech (Bristol, Avon), vol. 20, pp. 591-599.

MATEO, S., ROBY-BRAMI, A., REILLY, K.T., ROSSETTI, Y., COLLET, C., y RODE, G. (2015). "Upper limb kinematics after cervical spinal cord injury: a review". Journal of neuroengineering and rehabilitation, 12(1), 9 .

MCKINLEY, W., SANTOS, K., MEADE, M., BROOKE K. (2007). "Incidence and Outcomes of Spinal Cord Injury Clinical Syndromes”. J Spinal Cord Med. 2007;30:215-224

MURGIA, A., KYBERD, P., BARNHILLl, T., (2010). "The use of kinematic and parametric information to highlight lack of movement and compensation in the upper extremities during activities of daily living". Gait \& Posture, vol. 31, pp. 300-306.

MURPHY, M.A., WILLEN, C., SUNNERHAGEN, K.S., (2013). "Responsiveness of upper extremity kinematic measures and clinical improvement during the first three months after stroke". Neurorehabil Neural Repair, vol. 27, p. 844.

MURPHY, M.A., WILLEN, C., SUNNERHAGEN, K.S., (2011). "Kinematic variables quantifying upper extremity performance after stroke during reaching and drinking from a glass". Neurorehabil Neural Repair, vol. 25(1), pp. 71-80.

MURPHY, M.A., SUNNERHAGEN, K.S., JOHNELS, B., y WILLÉN, C. (2006). "Threedimensional kinematic motion analysis of a daily activity drinking from a glass: a pilot study". Journal of neuroengineering and rehabilitation, 3(1), 18.

NAMDARI, S., YAGNIK, G., EBAUGH, D.D., NAGDA, S., RAMSEY, M.L., WILLIAMS Jr, G.R., MEHTA, S.(2012). "Defining functional shoulder range of motion for activities of daily living". Journal of Shoulder and Elbow Surgery, vol. 21, no. 9, pp. 1177-1183.

OSU, R., OTA, K., FUJIWARA, T., OTAKA, Y., KAWATO M., LIU, M., (2011). "Quantifying the quality of hand movement in stroke patients through three-dimensional curvature". J Neuroeng rehabil, 8:62. 
PARKER, V.M., WADE, D.T., y HEWER, R.L. (1986). "Loss of arm function after stroke: measurement, frequency, and recovery”. International rehabilitation medicine, 8(2), 69-73.

PETUSKEY, K., BAGLEY, A., ABDALA, E., JAMES, M., RAB, G., (2007). "Upper extremity kinematics during functional activities: Three-dimensional studies in a normal pediatric population". Gait Posture, vol. 25, pp. 573-579.

SCHNEIDER R, THOMPSON J, BEBIN J (1958) "The syndrome of acute central cervical spinal cord Injury". J Neurol Neurosurg Psychiat 21:216-227.

VAN ANDEL, C.J., WOLTERBEEK, N., DOORENBOSCH, C.A., VEEGER, D.H., HARLAAR, J., (2008). "Complete 3D kinematics of upper extremity functional tasks". Gait Posture, vol. 27, pp. 120- 127.

WU, G., VAN DER HELM, F.C., VEEGER, H.D., MAKHSOUS, M., VAN ROY, P., ANGLIN, C. y WERNER, F.W. (2005). "ISB recommendation on definitions of joint coordinate systems of various joints for the reporting of human joint motion-Part II: shoulder, elbow, wrist and hand". Journal of biomechanics, 38(5), 981-992.

WYNDAELE, M., y WYNDAELE, J.J. (2006). "Incidence, prevalence and epidemiology of spinal cord injury: what learns a worldwide literature survey?". Spinal cord, 44(9), 523. 


\title{
Cambios en la cinemática articular tras entrenamiento de la marcha con exoesqueleto robótico ambulatorio
}

\author{
del-Ama, Antonio J. ${ }^{\text {a }}$, Megía, Álvaro ${ }^{a}$; Lozano-Berrio, Vicente ${ }^{a}$; Gil-Agudo, Ángel ${ }^{\text {b }}$ \\ ${ }^{a}$ Unidad de Biomecánica y Ayudas Técnicas. Hospital Nacional de Parapléjicos -Finca La Peraleda \\ S/N, Toledo, España. ajdela@sescam.jccm.es, \\ ${ }^{\mathrm{b}}$ Servicio de Rehabilitación. Hospital Nacional de Parapléjicos -Finca La Peraleda S/N, Toledo, \\ España.
}

\begin{abstract}
Ambulatory robotic exoskeletons for intensive gait training have been proposed as alternate to improve patient involvement. In this article, a case study is presented in which the effect of exoskeleton-assisted gait training on lower limb joint kinematics during 4 training sessions is assessed. The results suggest changes in joint kinematics during exoskeleton walking training sessions. These changes tend to be reduced as the training sessions increase, although it has not been possible to demonstrate statistically. The experimental procedure can be considered valid, this study being the basis for a broader analysis with a greater number of subjects and training sessions.
\end{abstract}

Keywords: gait, biomechanics, Robotics, exoskeletons, sensors

\section{Resumen}

Los exoesqueletos robóticos ambulatorios para el entrenamiento intensivo de la marcha, asegurando la involucración del paciente en la tarea. En este artículo se presenta un estudio piloto sobre el efecto del entrenamiento de la marcha asistida con exoesqueleto sobre la cinemática de las articulaciones de la extremidad inferior de un único paciente durante 4 sesiones de entrenamiento con exoesqueleto ambulatorio. Los resultados sugieren cambios en la cinemática articular durante las sesiones de entrenamiento de la marcha con exoesqueleto. Los cambios tienden a reducirse según aumentan las sesiones de entrenamiento, aunque no se ha podido demostrar estadísticamente. El procedimiento experimental puede considerarse válido, siendo este estudio la base para un análisis más amplio con un mayor número de sujetos y sesiones de entrenamiento.

Palabras clave: marcha, biomecánica, robótica, exoesqueletos, sensores inerciales. 


\section{Introducción}

La afectación de la función de marcha tras una lesión medular es una de las principales secuelas tras la lesión medular. Se estima que aproximadamente la mitad de las plesiones medulares son incompletas, cuya afectación de las extremidades es variable [1]. La rehabilitación de la capacidad de marcha es uno de los objetivos principales de las personas con lesión medular [2]

Actualmente la reeducación de la marcha se fundamenta en los principios de la promoción de mecanismos neuroplásticos mediante la ejecución de tareas motoras de manera repetitiva e intensiva. La premisa es proporcionar al sistema nervioso dañado la información sensorial adecuada para estimular las redes neuronales intactas de la médula espinal para facilitar su activación, incluso cuando la parte supra-espinal está comprometida. Los circuitos neuronales responden a las activaciones sensoriales, modulando el movimiento, pudiendo inducir modificaciones permanentes con la repetición [19]. La repetición intensiva del movimiento de la marcha, descargando parcialmente el peso del paciente, ha sido el principal paradigma de entrenamiento que sigue estos principios [20]. Debido a la demanda física que este entrenamiento impone a los fisioterapeutas, a principios de los años 200 se desarrolló un robot capaz de realizar la movilización de las extremidades inferiores sobre un tapiz rodante con descarga parcial de peso, denominado Lokomat [21] La utilización de Lokomat permite incrementar la intensidad y duración del entrenamiento, si bien este incremento no se ha correspondido con un incremento en los resultados funcionales obtenidos por los grupos de pacientes [8], [10]. Hay algunos autores que sugieren que los motivos de esta aparente falta de eficacia residen en que este tipo de robots eliminan la variabilidad intrínseca de la marcha, un mecanismo conocido del aprendizaje motor, así como la involucración del paciente en la tarea [12]

Para mejorar las condiciones de entrenamiento de la marcha, los exoesqueletos robóticos ambulatorios constituyen una alternativa que permite asimismo el entrenamiento intensivo de la marcha, asegurando la involucración del paciente en la tarea, a cambio de un mayor coste metabólico y la consecuente reducción en el tiempo de tratamiento [22], [23]. Si bien existe evidencia sobre su seguridad y tolerancia, actualmente se está investigando su efecto terapéutico para la rehabilitación de la marcha [24]-[26]. Dada la similitud con los entrenadores robóticos de la marcha, se ha asumido que los mecanismos terapéuticos son los mismos en ambos sistemas, si bien los mecanismos biomecánicos y fisiológicos de la marcha ambulatoria asistida por robot no son exactamente los mismos [27]. Es necesario por tanto investigar los mecanismos de adaptación entre el usuario y el exoesqueleto, el efecto sobre los sistemas nerviosos central y periférico, las estrategias de compensación 
generadas por el usuario en respuesta a la asistencia y el impacto físiológico del entrenamiento de la marcha asistida por exoesqueleto.

En este artículo se presenta un estudio piloto sobre el efecto del entrenamiento de la marcha asistida con exoesqueleto sobre la cinemática de las articulaciones de la extremidad inferior. La hipótesis principal es que el entrenamiento de la marcha por un tiempo de 15 minutos provoca cambios inmediatos en la cinemática articular. Para ello, se plantea como objetivo analizar la cinemática de las articulaciones de la cadera, rodilla tobillo inmediatamente antes y después del entrenamiento de la marcha con exoesqueleto ambulatorio.

\section{Material y métodos.}

\subsection{Paciente: patología y afectación de la marcha.}

La paciente es una mujer de 51 años de edad con síndrome de lesión medular L1 ASIA C. Al inicio del programa de entrenamiento de la marcha con exoesqueleto presenta un índice muscular del miembro inferior de 16/14 (izquierda/derecha) sobre 25, espasticidad Ashworth $=0$ y $P E N=1$. La valoración funcional de la marcha responde a los siguientes valores: Test de los 10 metros $=36$ segundos; test de los 6 minutos $=99$ metros; Timed-upand-go $=43$ segundos; WISCI-II = 9; SCIM-III = 71/100.

La paciente deambula con andador sin supervisión con órtesis de tobillo en pie derecho para interiores. Usa silla de ruedas con propulsión manual para espacios exteriores.

\subsection{Exoesqueleto HANK}

El exoesqueleto HANK (Gogoa Mobility, Traña-Matiena, Bizkaia) Su diseño se basa en una estructura ortésica con una única barra lateral de soporte, con 3 grados de libertad ubicados en el plano lateral de la barra, correspondiendo con los actuadores para las caderas, rodillas y tobillos. Los actuadores están compuestos por motores de corriente continua sin escobillas y reductores Harmonic Drive, con relación de transmisión 160:1, encóders magnéticos y sensores de fuerza. Los actuadores integran una placa con la electrónica de control de los motores, la adquisición y tratamiento de las señales de los sensores, así como la comunicación con el controlador principal.

La arquitectura de control del exoesqueleto se basa en un microcontrolador que permite la comunicación mediante Bluethooth con la interfaz de control externa. Integra también un bus de comunicación CAN para mandar las consignas de control a los actuadores, así como recibir la información de los sensores. 
La interfaz de control se implementa mediante una aplicación instalada en una tablet o teléfono móvil con sistema Android. Permite varias acciones y modos de control. Para las sesiones de entrenamiento se han utilizado las siguientes: levantar, marcha continua, cambio de velocidad de la marcha, detención, y sentado.

\subsection{Sensores inerciales}

Para el registro del movimiento articular se utilizó un conjunto de sensores inerciales de TechnAid (Arganda del Rey, Madrid), compuestos por acelerómetro, giróscopo y magnetrómetro dispuestos ortogonalmente. Mediante un algoritmo de fusión sensorial basado en un filtro de Kalman modificado, los sensores estiman su orientación en el espacio respecto a un sistema de referencia no inercial constituido por la gravedad y el norte magnético terrestre. La comunicación entre los sensores se realiza mediante un bus SPI que transmiten la información a un concentrador de datos que a su vez permite la transmisión de datos mediante Bluethoot a un ordenador externo.

\subsection{Protocolo experimental.}

\subsubsection{Registro de la cinemática de la marcha}

Previamente a realizar el entrenamiento, se colocaron 4 sensores inerciales colocados en el pie, tibia, muslo y pelvis de la paciente, utilizando los soportes y cinchas del fabricante. Este montaje permite el cálculo de los ángulos articulares del tobillo, rodilla y cadera. Tras la puesta de los sensores, se realizó una calibración de la orientación de los sensores respecto a los principales ejes anatómicos de los segmentos en los que se ubican los sensores, pidiendo a la paciente que mantenga una posición erguida con las articulaciones alineadas, durante 3 segundos, y registrando la orientación de los sensores. Tras este procedimiento, se pide a la paciente que comience a andar en línea recta, utilizando para ello un andador. Se realiza la captura de los datos durante al menos 2 minutos. Tras la captura, la paciente pasa a una silla, donde se quitan los sensores y se pone el exoesqueleto.

\subsubsection{Entrenamiento de la marcha con exoesqueleto}

El exoesqueleto se alinea con las articulaciones de la paciente y se ajustan las cinchas hasta conseguir una sujeción firme y no molesta. Se pasa a bipedestación con la asistencia del exoesqueleto y el andador de la paciente. Tras inspeccionar la alineación del exoesqueleto con las articulaciones de la paciente, y verificar las cinchas, se comienza con el entrenamiento de la marcha. 
El entrenamiento de la marcha consiste en la realización de 15 minutos de marcha asistida por el exoesqueleto en línea recta y ajustando la velocidad de la marcha a la capacidad de la paciente, bajo criterio del terapeuta responsable del entrenamiento.

Tras los 15 minutos de marcha, se retira el exoesqueleto y se vuelven a colocar los sensores inerciales, y la paciente vuelve a realizar el procedimiento descrito anteriormente. El tiempo entre la finalización del entrenamiento de la marcha y el registro de la cinemática de la marcha es inferior a 5 minutos.

\subsection{Análisis de datos}

Los datos de los sensores inerciales se descargaron y trataron mediante MATLAB. En primer lugar se segmentaron los datos mediante un algoritmo especialmente diseñado para este experimento, detectando el contacto inicial a través de la verificación de los siguientes eventos: extensión completa de la rodilla, cadera en extensión y tobillo en flexión dorsal. Además, el algoritmo permite realizar una inspección visual del resultado de la detección, facilitando eliminar detecciones incorrectas.

Tras la detección se extrajeron las trayectorias articulares en el plano sagital de cada paso y se normalizaron entre 0 y $100 \%$. Para cada paso y articulación se obtuvieron obtienen las siguientes variables: máximo, mínimo, rango articular, y valor cuadrático medio. Además, para cada articulación y grado de libertad se obtuvo la curva resultante de promediar todos los pasos.

Se realizó una prueba t de Student para evaluar las diferencias entre las variables obtenidas previamente y tras cada entrenamiento de la marcha, así como entre el registro inicial del primer y último entrenamiento de la marcha. El valor de significación se estableció en $\mathrm{p}<0,05$.

\section{Resultados.}

La figura 1 muestra los la trayectoria cinemática promedio en el plano sagital para las articulaciones de la cadera, rodilla y tobillo inmediatamente antes (curva azul) y después (rojo) de realizar entrenamiento de la marcha durante 15 minutos con el exoesqueleto HANK para las 4 sesiones consideradas. La última fila de la Figura 1 muestra la comparación de los registros previos la tratamiento de las sesiones 1 y 4 . Las principales variables descriptoras, máximo, mínimo, rango de movimiento y valor promedio RMS correspondientes a las gráficas se muestran en las tablas 1 a 3, para las articulaciones de cadera, rodilla y tobillo respectivamente. 
Cambios en la cinemática articular tras entrenamiento de la marcha con exoesqueleto robótico ambulatorio
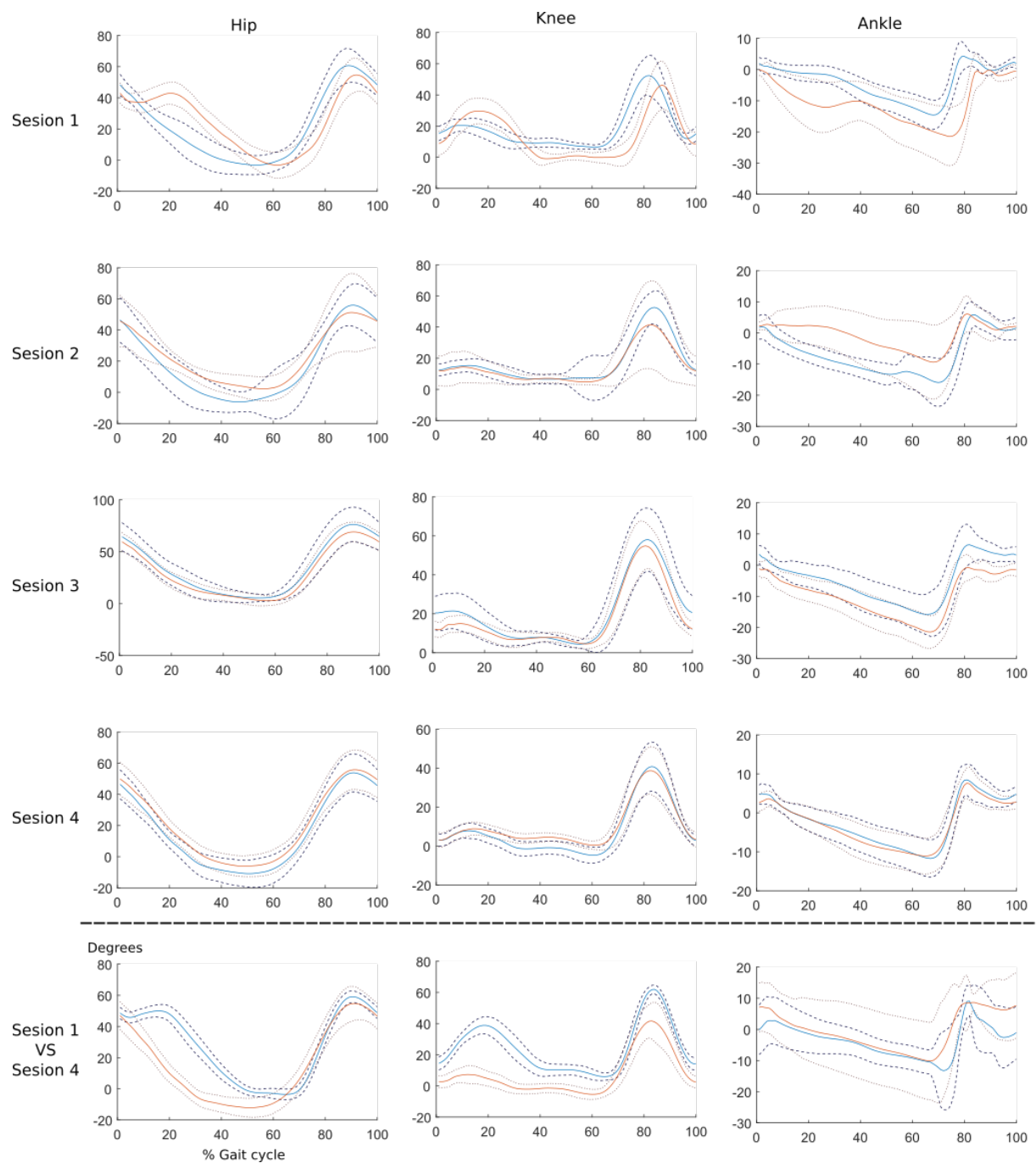

Fig. 1: Angulo de flexión-extensión de las articulaciones de la cadera, rodilla y tobillo (primera, segunda y tercera fila respectivamente) obtenidos inmediatamente antes (curva azul) y después (curva roja) de 15 minutos de entrenamiento de la marcha con el exoesqueleto HANK. La última fila corresponde a la comparación entre las sesiones primera y última de entrenamiento. Las curvas están normalizadas de 0 a $100 \%$ del ciclo de la marcha y representan un ciclo promedio \pm desviación estándar.

La inspección visual de las gráficas muestra diferencias apreciables entre el registro previo y posterior al entrenamiento en la sesión 1, siendo esta diferencia menos apreciable según avanzan las sesiones de tratamiento, llegando a ser casi coincidentes en la sesión. La progresión del paciente en la modificación de las trayectorias articulares se muestra en la 
última fila de la Figura 1, donde se muestra un cambio apreciable en las trayectorias articulares.

Sin embargo, la inspección de los descriptores de las gráficas muestra que siguen existiendo diferencias en los parámetros analizados, por lo que sigue existiendo un efecto del uso del exoesqueleto en el paciente $(\mathrm{p}<0.05)$.

Tabla 1: Parámetros cinemáticos promedio de la flexión-extensión de la cadera. Los datos son media \pm desviación estándar. Los valores en negrita representan significación estadística entre pre- y postentrenamiento para una misma sesión. El * indica significación estadística entre las sesiones 1 y 4.

\begin{tabular}{ll|cccc} 
& & Máximo & Mínimo & $\begin{array}{c}\text { Rango } \\
\text { articular }\end{array}$ & RMS \\
\hline \multirow{2}{*}{ Sesión 1 } & Pre & $59,5 \pm 3.4$ & $\mathbf{- 8 . 6 \pm 3 . 7 *}$ & $\mathbf{6 8 , 1} \pm \mathbf{5 . 4}$ & $\mathbf{3 1 , 0} \pm \mathbf{2 . 9}$ \\
& Post & $58,3 \pm 3.0$ & $\mathbf{- 6 , 9} \pm \mathbf{2 . 8}$ & $\mathbf{6 5 , 1} \pm \mathbf{3 . 2}$ & $\mathbf{3 3 , 3} \pm \mathbf{1 . 9}$ \\
\hline \multirow{2}{*}{ Sesión 2 } & Pre & $\mathbf{5 9 , 9} \pm \mathbf{3 . 1}$ & $\mathbf{- 9 , 4} \pm \mathbf{3 . 9}$ & $69,3 \pm 4.3$ & $\mathbf{3 0 , 5} \pm \mathbf{2 . 6}$ \\
& Post & $\mathbf{6 9 , 2} \pm \mathbf{3 . 0}$ & $\mathbf{- 3 , 1} \pm \mathbf{3 . 6}$ & $72,4 \pm 3.4$ & $\mathbf{3 6 , 1} \pm \mathbf{2 . 2}$ \\
\hline \multirow{2}{*}{ Sesión 3 } & Pre & $\mathbf{8 2 , 7 \pm 3 , 2}$ & $\mathbf{4 , 0} \pm \mathbf{2 , 5}$ & $\mathbf{7 8 , 6} \pm \mathbf{3 , 7}$ & $\mathbf{4 4 , 5} \pm \mathbf{3 , 6}$ \\
& Post & $\mathbf{7 1 , 7} \pm \mathbf{2 , 3}$ & $\mathbf{1 , 7} \pm \mathbf{4 , 0}$ & $\mathbf{7 0 , 0} \pm \mathbf{4 , 2}$ & $\mathbf{3 8 , 7} \pm \mathbf{2 , 1}$ \\
\hline \multirow{2}{*}{ Sesión 4 } & Pre & $57,7 \pm 2,9$ & $\mathbf{- 1 3 , 8} \pm \mathbf{4 , 8 *}$ & $71,5 \pm 4,0^{*}$ & $29,8 \pm 1,9$ \\
& Post & $60,0 \pm 1,6$ & $\mathbf{- 8 , 5} \pm \mathbf{3 , 5}$ & $68,5 \pm 3,2$ & $32,2 \pm 1,3$ \\
\hline
\end{tabular}

Tabla 2: Parámetros cinemáticos promedio de la flexión-extensión de la rodilla. Los datos son media \pm desviación estándar. Los valores en negrita representan significación estadística entre pre- y postentrenamiento para una misma sesión. El * indica significación estadística entre las sesiones 1 y 4.

\begin{tabular}{|c|c|c|c|c|c|}
\hline & & Máximo & Mínimo & $\begin{array}{c}\text { Rango } \\
\text { articular }\end{array}$ & RMS \\
\hline \multirow{2}{*}{ Sesión 1} & Pre & $47,0 \pm 2.1 *$ & $0,9 \pm 2.3^{*}$ & $68,1 \pm 5.4^{*}$ & $18,1 \pm 1.7$ \\
\hline & Post & $52,4 \pm 3.8$ & $-3,5 \pm 1.8$ & $65,1 \pm 3.2$ & $22,4 \pm 2.2$ \\
\hline \multirow{2}{*}{ Sesión 2} & Pre & $57,3 \pm 1.9$ & $1,8 \pm 1.8$ & $69,3 \pm 4.3$ & $23,6 \pm 1.9$ \\
\hline & Post & $62,7 \pm 2.2$ & $4,6 \pm 1.5$ & $72,4 \pm 3.4$ & $27,1 \pm 1.5$ \\
\hline \multirow{2}{*}{ Sesión 3} & Pre & $66,2 \pm 3.0$ & $3,5 \pm 1.2$ & $78,6 \pm 3.7$ & $29,0 \pm 2.5$ \\
\hline & Post & $59,9 \pm 2.8$ & $3,4 \pm 1.6$ & $70,0 \pm 4.2$ & $24,7 \pm 1.4$ \\
\hline \multirow{2}{*}{ Sesión 4} & Pre & $45,3 \pm 1.8 *$ & $-6,3 \pm 2.6 *$ & $71,5 \pm 4.0 *$ & $17,2 \pm 0.9$ \\
\hline & Post & $43,6 \pm 1.9$ & $-1,0 \pm 2.4$ & $68,5 \pm 3.2$ & $16,9 \pm 1.0$ \\
\hline
\end{tabular}


Cambios en la cinemática articular tras entrenamiento de la marcha con exoesqueleto robótico ambulatorio

Tabla 3: Parámetros cinemáticos promedio de la flexión-extensión de la tobillo. Los datos son media \pm desviación estándar. Los valores en negrita representan significación estadística entre pre- y postentrenamiento para una misma sesión. El * indica significación estadística entre las sesiones 1 y 4.

\begin{tabular}{ll|cccc} 
& & Máximo & Mínimo & $\begin{array}{c}\text { Rango } \\
\text { articular }\end{array}$ & RMS \\
\hline \multirow{2}{*}{ Sesión 1 } & Pre & $7,8 \pm 1.0^{*}$ & $\mathbf{- 1 5 , 7 \pm 0 . 9 *}$ & $68,1 \pm 5.4^{*}$ & $\mathbf{8 , 5} \pm \mathbf{1 . 0}$ \\
& Post & $2,7 \pm 1.9$ & $\mathbf{- 2 5 , 1} \pm \mathbf{2 . 6}$ & $65,1 \pm 3.2$ & $\mathbf{1 4 , 4} \pm \mathbf{2 . 0}$ \\
\hline \multirow{2}{*}{ Sesión 2 } & Pre & $\mathbf{8 , 7} \pm \mathbf{1 . 5}$ & $\mathbf{- 1 9 , 8} \pm \mathbf{1 . 7}$ & $\mathbf{6 9 , 3} \pm \mathbf{4 . 3}$ & $\mathbf{1 0 , 2} \pm \mathbf{1 . 3}$ \\
& Post & $\mathbf{1 0 , 7} \pm \mathbf{1 . 0}$ & $\mathbf{- 1 8 , 6} \pm \mathbf{0 . 8}$ & $\mathbf{7 2 , 4} \pm \mathbf{3 . 4}$ & $\mathbf{8 , 6} \pm \mathbf{0 . 5}$ \\
\hline \multirow{2}{*}{ Sesión 3 } & Pre & $\mathbf{1 1 , 3} \pm \mathbf{1 . 0}$ & $\mathbf{- 1 9 , 1} \pm \mathbf{1 . 9}$ & $\mathbf{7 8 , 6} \pm \mathbf{3 . 7}$ & $\mathbf{9 , 9} \pm \mathbf{0 . 9}$ \\
& Post & $\mathbf{1 , 9} \pm \mathbf{2 . 4}$ & $\mathbf{- 2 3 , 3} \pm \mathbf{1 . 8}$ & $\mathbf{7 0 , 0} \pm \mathbf{4 . 2}$ & $\mathbf{1 2 , 7} \pm \mathbf{1 . 1}$ \\
\hline \multirow{2}{*}{ Sesión 4 } & Pre & $11,1 \pm 0.9 *$ & $-13,4 \pm 1.1^{*}$ & $71,5 \pm 4.0^{*}$ & $7,5 \pm 0.5^{*}$ \\
& Post & $9,8 \pm 0.7$ & $-12,6 \pm 1.1$ & $68,5 \pm 3.2$ & $7,5 \pm 0.5$ \\
\hline
\end{tabular}

\section{Discusión.}

La utilización de un exoesqueleto ambulatorio para el entrenamiento de la marcha se fundamenta en los principales principios de la neurorrehabilitación: la realización de tareas funcionales, repetitivas e intensivas, con el objetivo de fomentar los mecanismos neuroplásticos y de aprendizaje que permitan la recuperación de la función alterada.

Hasta la fecha no existen estudios que hayan investigado los cambios cinemáticos inmediatos al entrenamiento con exoesqueleto. Este estudio es el primero que explora los posibles cambios cinemáticos en el plano sagital del entrenamiento de la marcha con exoesqueleto ambulatorio. Los resultados presentados muestran que efectivamente parece que existe un cierto efecto posterior al entrenamiento, que parece instaurarse en el paciente a través de las 4 sesiones analizadas. La inspección de las curvas muestra una adaptación progresiva hacia los patrones finales, similares a los patrones cinemáticos impuestos por el exoesqueleto, que provienen a su vez de una base de datos normativa [28]. No obstante, el análisis estadístico realizado a los descriptores de las curvas muestra diferencias significativas en todas las sesiones, incluso en la sesión 4, donde las curvas aparentemente muestran una gran igualdad.

Este trabajo abre numerosas cuestiones importantes para el campo de la neurorrehabilitación de la marcha con robots de asistencia. En primer lugar, es necesario estudiar si existen cambios en las articulaciones en los planos frontal y coronal como consecuencia de la restricción de movimiento del exoesqueleto. En este trabajo se ha 
obviado este análisis debido a la extensión del mismo, y porque a priori no cabe esperar demasiados cambios en estos grados de libertad dado que no son movilizados por el robot.

El efecto de la intensidad de la terapia en el efecto tras entrenamiento debe ser estudiado. La pregunta subyacente es si existe una dosis o tiempo de entrenamiento por encima del cual no se producen cambios en la cinemática articular, siendo entonces innecesario continuar el entrenamiento en esa sesión.

Este estudio cuenta con varias limitaciones. En primer lugar, el bajo número de sesiones de entrenamiento analizado, así como las características particulares de la paciente impide obtener conclusiones generalizables sobre el proceso de aprendizaje. Además, la adquisición de la cinemática mediante los sensores inerciales presenta limitaciones vinculadas a las prestaciones de esta tecnología y el procedimiento de registro. La tecnología de medida ambulatoria inercial presenta limitaciones relacionadas con la deriva de los sensores y las perturbaciones electromagnéticas que limitan la precisión de las muestras obtenidas. Por otra parte, la calibración anatómica de la orientación de los sensores a la orientación de los segmentos introduce también alteraciones en las medidas.

\section{Conclusión.}

El presente estudio ha mostrado que existen cambios en la cinemática articular durante las sesiones de entrenamiento de la marcha con exoesqueleto. Los cambios tienden a reducirse según aumentan las sesiones de entrenamiento, aunque no se ha podido demostrar estadísticamente. El procedimiento experimental puede considerarse válido, siendo este estudio la base para un análisis más amplio con un mayor número de sujetos y sesiones de entrenamiento.

\section{Agradecimientos.}

Este trabajo ha sido posible gracias a los proyectos Bidirectional Hyper-Connected Neural System (EXTEND), del programa de investigación e innovación H2020, (Ref. 779982), y Sistemas Modulares Robóticos y Neuroprotésicos Personalizables para la Asistencia de la Marcha Patológica (TAILOR), financiado por el Ministerio de Ciencia, Innovación y Universidades, dentro del programa de Proyectos de I+D+i Retos de Investigación (Ref. RTI2018-097290-B-C31) 


\section{Referencias.}

[1] M. Wyndaele and J. J. Wyndaele, "Incidence, prevalence and epidemiology of spinal cord injury: What learns a worldwide literature survey?," Spinal Cord, vol. 44, no. 9, pp. 523-529, 2006.

[2] P. L. Ditunno, M. Patrick, M. Stineman, and J. F. Ditunno, "Who wants to walk? Preferences for recovery after SCI: A longitudinal and cross-sectional study," Spinal Cord, vol. 46, no. 7, pp. 500-506, 2008.

[3] G. Colombo, M. Wirz, and V. Dietz, "Driven gait orthosis for improvement of locomotor training in paraplegic patients," in Spinal Cord, 2001, vol. 39, no. 5, pp. 252-255.

[4] G. Colombo, M. Joerg, R. Schreier, and V. Dietz, "Treadmill training of paraplegic patients using a robotic orthosis," J. Rehabil. Res. Dev., vol. 37, no. 6, pp. 693-700, 2000.

[5] S. Freivogel, J. Mehrholz, T. Husak-Sotomayor, and D. Schmalohr, "Gait training with the newly developed'LokoHelp'-system is feasible for non-ambulatory patients after stroke, spinal cord and brain injury. A feasibility study," Brain Inj., vol. 22, no. 7-8, pp. 625-632, 2008.

[6] S. Hesse, A. Waldner, and C. Tomelleri, "Innovative gait robot for the repetitive practice of floor walking and stair climbing up and down in stroke patients," J. Neuroeng. Rehabil., vol. 7, no. 1, p. 30, Jan. 2010.

[7] C. Nooijen, N. Ter Hoeve, and E. Field-Fote, "Gait quality is improved by locomotor training in individuals with SCI regardless of training approach," J. Neuroeng. Rehabil., vol. 6, no. 1, p. 36, Jan. 2009.

[8] C. Morawietz and F. Moffat, "Effects of locomotor training after incomplete spinal cord injury: A systematic review," Arch. Phys. Med. Rehabil., vol. 94, no. 11, pp. 2297-2308, 2013.

[9] M. Wessels, C. Lucas, I. Eriks-Hoogland, and S. De Groot, "Body weight-supported gait training for restoration of walking in people with an incomplete spinal cord injury: A systematic review," Assist. technol. Res. Ser., vol. 26, no. 6, pp. 297-299, Jun. 2010.

[10] E. Swinnen, S. Duerinck, J. Baeyens, R. Meeusen, and E. Kerckhofs, "Effectiveness of robotassisted gait training in persons with spinal cord injury: A systematic review," J. Rehabil. Med., vol. 42, no. 6, pp. 520-526, Jun. 2010.

[11] J. Mehrholz, J. Kugler, and M. Pohl, "Locomotor training for walking after spinal cord injury," Cochrane database Syst. Rev., vol. 11, no. 11, p. CD006676, 2012.

[12] B. H. Dobkin and P. W. Duncan, "Should body weight-supported treadmill training and roboticassistive steppers for locomotor training trot back to the starting gate?," Neurorehabil. Neural Repair, vol. 26, no. 4, pp. 308-317, May 2012.

[13] A. Wall, J. Borg, and S. Palmcrantz, "Clinical application of the Hybrid Assistive Limb (HAL) for gait training: a systematic review," Front. Syst. Neurosci., vol. 9, no. 9, pp. 483389-48, 2015.

[14] M. Bortole et al., "The H2 robotic exoskeleton for gait rehabilitation after stroke: Early findings from a clinical study Wearable robotics in clinical testing," J. Neuroeng. Rehabil., vol. 12, no. 1, pp. 1-14, 2015.

[15] V. Lajeunesse, C. Vincent, F. Routhier, E. Careau, and F. Michaud, "Exoskeletons' design and usefulness evidence according to a systematic review of lower limb exoskeletons used for 
functional mobility by people with spinal cord injury," Disabil. Rehabil. Assist. Technol., vol. 11, no. 7, pp. 535-547, 2016.

[16] S. Federici, F. Meloni, M. Bracalenti, and M. L. De Filippis, "The effectiveness of powered, active lower limb exoskeletons in neurorehabilitation: A systematic review," NeuroRehabilitation, vol. 37, no. 3, pp. 321-340, 2015.

[17] J. L. Contreras-Vidal et al., "Powered exoskeletons for bipedal locomotion after spinal cord injury," J. Neural Eng., vol. 13, no. 3, pp. 1-16, 2016.

[18] D. R. Louie, J. J. Eng, and T. Lam, "Gait speed using powered robotic exoskeletons after spinal cord injury: A systematic review and correlational study," J. Neuroeng. Rehabil., vol. 12, no. 1, p. 82, 2015.

[19] S. J. Harkema, J. Hillyer, M. Schmidt-Read, E. Ardolino, S. A. Sisto, and A. L. Behrman, "Locomotor Training: As a treatment of spinal cord injury and in the progression of neurologic rehabilitation," Arch. Phys. Med. Rehabil., vol. 93, no. 9, pp. 1588-1597, 2012.

[20] V. Dietz, "Body weight supported gait training: From laboratory to clinical setting," Brain Research Bulletin, vol. 76, no. 5. pp. 459-463, 2008.

[21] S. Jezernik, G. Colombo, T. Keller, H. Frueh, and M. Morari, "Robotic Orthosis Lokomat: A Rehabilitation and Research Tool," Neuromodulation, vol. 6, no. 2, pp. 108-115, 2003.

[22] N. Evans, C. Hartigan, C. Kandilakis, E. Pharo, and I. Clesson, "Acute Cardiorespiratory and Metabolic Responses During Exoskeleton-Assisted Walking Overground Among Persons with Chronic Spinal Cord Injury,” Top. Spinal Cord Inj. Rehabil., vol. 21, no. 2, pp. 122-132, 2015.

[23] N. Lefeber, E. Swinnen, M. Michielsen, S. Henderix, and E. Kerckhofs, "Energy consumption and cardiorespiratory load during lokomat walking compared to walking without robotassistance in stroke patients: Preliminary results," in Biosystems and Biorobotics, vol. 15, J. Ibáñez, J. González-Vargas, J. M. Azorín, M. Akay, and J. L. Pons, Eds. Cham: Springer International Publishing, 2017, pp. 1229-1233.

[24] A. Esquenazi, M. Talaty, and A. Jayaraman, "Powered Exoskeletons for Walking Assistance in Persons with Central Nervous System Injuries: A Narrative Review," $P M R$, vol. 9, no. 1, pp. 46-62, Jan. 2017.

[25] L. J. Holanda, P. M. M. Silva, T. C. Amorim, M. O. Lacerda, C. R. Simão, and E. Morya, "Robotic assisted gait as a tool for rehabilitation of individuals with spinal cord injury: a systematic review," J. Neuroeng. Rehabil., vol. 14, no. 1, p. 126, 2017.

[26] E. Y. Y. Cheung, T. K. W. Ng, K. K. K. Yu, R. L. C. Kwan, and G. L. Y. Cheing, "RobotAssisted Training for People With Spinal Cord Injury: A Meta-Analysis," Arch. Phys. Med. Rehabil., vol. 98, no. 11, pp. 2320-2331.e12, 2017.

[27] F. Sylos-Labini et al., "EMG patterns during assisted walking in the exoskeleton," Front. Hum. Neurosci., vol. 8, no. June, p. 423, 2014.

[28] C. A. Fukuchi, R. K. Fukuchi, and M. Duarte, "A public dataset of overground and treadmill walking kinematics and kinetics in healthy individuals," PeerJ, vol. 6, p. e4640, 2018. 


\section{ROBÓTICAE IMAGEN MÉDICA}




\title{
Sistema no invasivo para la medida y visualización de desplazamientos de tejidos en neurocirugía
}

\author{
Juan, Carlos G.; Blanco-Angulo, Carolina; Bermejo, Natividad; García, Hector; \\ Vicente, Jose María; Avila, Ernesto; Sabater-Navarro, Jose María \\ Grupo de Neuroingeniería Biomédica de la Universidad Miguel Hernández de Elche.
}

\begin{abstract}
A medical imaging system based on measuring distances between tissues by means of microwaves is presented. The system is designed for the monitoring and correction of the brain-shift problem in brain tumor operations. It shows the developed system and the first results with phantoms of brain tissues that have a morphology similar to real tissues. The viability of the system for the measurement of distances and the reconstruction of an intraoperative $3 D$ image is demonstrated.
\end{abstract}

Keywords: bioengineering, brain-shift, technology, microwave.

\section{Resumen}

Se presenta un sistema de imagen médica basado en medición de distancias entre tejidos por medio de microondas. El sistema está pensado para la monitorización y corrección del problema de brain-shift en operaciones de tumores cerebrales. Se muestra el sistema desarrollado y los primeros resultados con phantoms de tejidos cerebrales que tienen una morfología similar a los tejidos reales. Se demuestra la viabilidad del sistema para la medida de distancias y la reconstrucción de una imagen $3 D$ intraoperatoria.

Palabras clave: bioingeniería, brain-shift, tecnología, microondas. 


\section{Introducción}

La neurocirugía robótica está sufriendo profundos cambios en los últimos tiempos, fruto principalmente de los avances en las técnicas de imagen médica (TAC, MRI, f-MRI o DTI), lo que permite una mejor planificación de la operación a realizar. La neurocirugía mínimamente invasiva se ve beneficiada de estos avances. Sin embargo, quedan problemas a resolver en la transferencia del plan de trabajo preplanificado a la realidad intraoperatoria, debido a la naturaleza no lineal de los tejidos deformables involucrados. Uno de estos problemas es el brain-shift, o desplazamiento de la materia cerebral fruto del cambio de presión interior al practicar la craneotomía y de los propios procesos quirúrgicos, y que produce una pérdida de referencia entre los volúmenes de imagen de neurocirugía adquiridos antes de la cirugía.

Diversos autores han propuesto soluciones para abordar este problema. En (Letteboer, 2005) se propone el uso de un sistema de ecografía 3D que permita obtener una imagen 3D del volumen del cerebro. Otras aproximaciones han hecho uso de sistemas ópticos (Comparetti, 2011) para trackear los puntos anatómicos, si bien esta aproximación tiene la limitación de que el marker debe ser visible. Más interesantes son las aproximaciones que hacen uso de un modelo matemático de deformaciones (DeLorenzo, 2012, Chen, 2013, Oldfield, 2011) para predecir los desplazamientos de todos los puntos del cerebro.

En este trabajo se presenta un sistema de imagen médica por microondas para detección y corrección del problema de brain-shift en operaciones de tumores cerebrales. Se presenta el sistema desarrollado, los componentes electrónicos de alta frecuencia y control que forman parte del mismo y el software que permite obtener las imágenes. El sistema de antenas permite obtener en tiempo real la localización de los puntos de control del modelo de deformaciones para la visualización utilizando realidad aumentada.

\section{Metodología}

El sistema propuesto para realizar las medidas de distancias entre la antena y los diferentes tejidos biológicos creaneales se basa en un sistema de antenas de radiofrecuencia (RF) que permiten crear una imagen médica mediante la aplicación de algortimos de reconstrucción de imagen médica. La Figura 1 muestra una imagen del set-up prototipo donde se observan los principales componentes. 


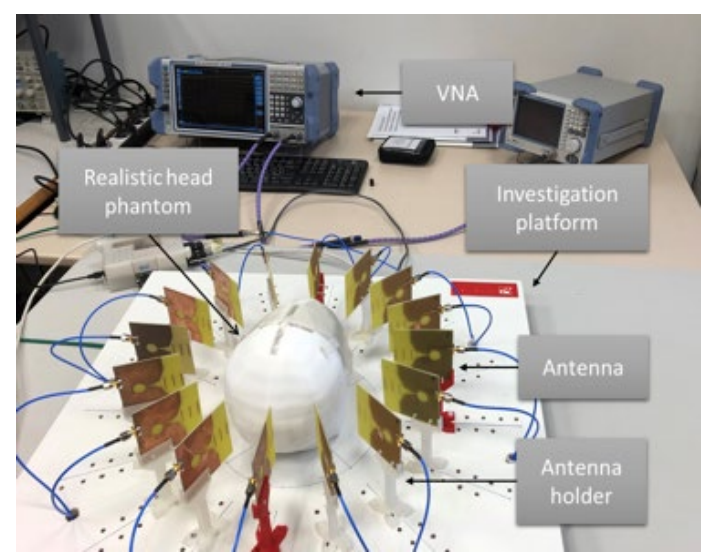

Fig. 1 Fotografía del sistema utilizado para obtener la imagen médica donde se pueden observar los distintos elementos que componen el sistema. Fuente: Elaboración propia (2019).

\subsection{Antenas diseñadas}

Las antenas, que se han diseñado y fabricado para el sistema de medida del proyecto son antenas tipo Vivaldi impresas en sustrato FR4 de $7 \mathrm{~cm}^{2}$. En la Figura 2 se muestran ambas caras de la antena diseñada. Como se puede observar, son antenas de tipo slot a la que se han añadido 3 elementos directores en su apertura y un radial stub $\lambda / 4$ en la alimentación. Estos elementos se han optimizado durante el proceso de diseño para obtener la mejor relación entre tamaño y diversos parámetros de la antena como ancho de banda, directividad y ganancia. En concreto los tres elementos directores situados en la apertura de la antena aumentan la directividad y ganancia, y se han diseñado para optimizar el diagrama de radiación a la frecuencia central de funcionamiento. Para alimentar la antena, como se puede ver en la imagen, se ha diseñado una línea microstrip seguida del radial stub $\lambda / 4$ que proporciona la adaptación de banda ancha. En este trabajo se utilizan 16 antenas colocadas formando un círculo y separadas $22.5^{\circ}$ entre sí respecto al centro de la circunferencia.
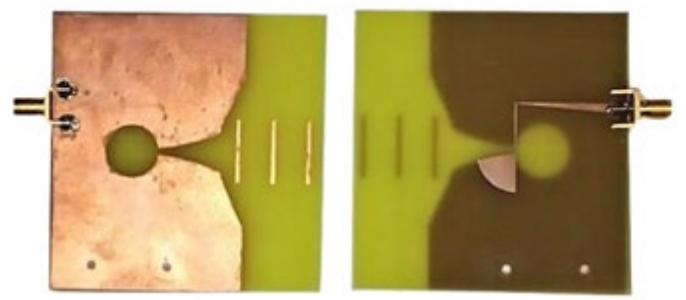

Fig. 2 Fotografía del prototipo de antena fabricado. Fuente: Elaboración propia (2018).

La antena se ha diseñado y simulado con el software EMPro. En la Figura 3 se muestra el parámetro S11 en el rango de funcionamiento, tanto de la simulación en EMPro como en la 
medida de la antena fabricada. Como se puede observar, la antena presenta un ancho de banda de funcionamiento de $1.2 \mathrm{GHz}$ a $5 \mathrm{GHz}$, medido para una respuesta por debajo de $-10 \mathrm{~dB}$.

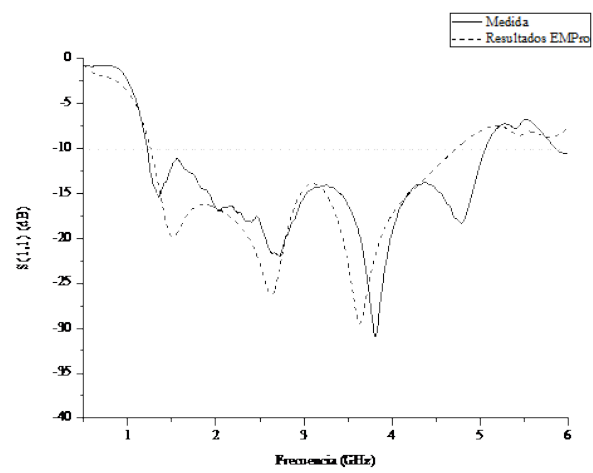

Fig. 3 Pérdidas de retorno de la antena fabricada. Fuente: Elaboración propia (2018).

En la Figura 4 se muestran los diagramas de radiación de la antena en plano E y plano $\mathrm{H}$, respectivamente. Estos diagramas se han representado para cinco frecuencias dentro del ancho de banda de funcionamiento de la antena (1, 2, 3, 4 y $5 \mathrm{GHz})$. Se puede extraer que la antena es prácticamente omnidireccional en el plano E en todo el rango. Por otro lado, en el plano $\mathrm{H}$ la antena es más directiva en la dirección de la apertura, gracias a los elementos directores colocados en esa dirección.

Por último, cabe comentar que la ganancia simulada con el software EMPro oscila entre 3 y $7 \mathrm{~dB}$ en todo el rango de frecuencias de funcionamiento de la antena.

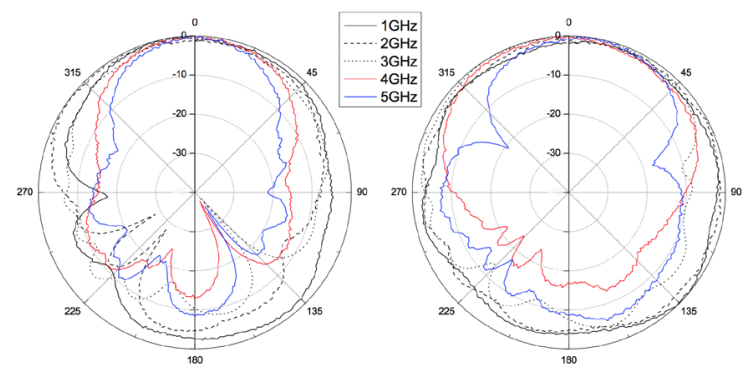

Fig. 4 Diagramas de radiación medidos de la antena en plano H (a) y E (b). Fuente: Elaboración propia (2018).

\subsection{Sistema de medida de distancias}

Debajo del tablero de madera que se observa en la Figura 1 se encuentra una red de 5 conmutadores de RF que seleccionan qué antena de las 16 utilizadas está conectada al VNA 
y mide en cada momento. Para las medidas se utiliza un phantom como el descrito en (Bermejo, 2018), donde se especifican las recetas para obtener phantoms de tejidos con propiedades dieléctricas en el rango de los tejidos biológicos, de forma que se pueda comprobar el funcionamiento del sistema desarrollado. La Tabla 1 muestra los parámetros de los tejidos phantoms utilizados.

Tabla 1. Parámetros dieléctricos de los phantoms utilizados.

\begin{tabular}{|c|c|c|}
\hline Materiales & $\boldsymbol{\varepsilon}^{\prime}$ & $\operatorname{tg} \boldsymbol{\delta}$ \\
\hline Piel & 44 & 0,64 \\
\hline Cerebro & 50 & 0,54 \\
\hline Tumor & 75 & 0,18 \\
\hline
\end{tabular}

\subsubsection{Commutadores de RF}

La conmutación de las antenas se realiza mediante conmutadores de RF. En concreto, se utilizan 5 conmutadores modelo ZSWA4-63DR+ de Mini-Circuits. Este tipo de conmutador se compone de una entrada y 4 salidas de RF que operan en la banda de $1 \mathrm{MHz}$ a $6 \mathrm{GHz}$ y están adaptadas a $50 \Omega$ en toda la banda.

El fabricante proporciona una tabla de verdad en la que relaciona la entrada con las salidas. En la tabla también se indican dos estados adicionales, uno de ellos es el estado de reposo de los conmutadores y el otro es un estado no soportado, el cual no debería producirse.

De estos cinco conmutadores, uno de ellos es el conmutador que controla los otros cuatro, formando una topología de estrella, siendo además el conmutador que se conecta al VNA para medir los resultados. Cada uno de los otros cuatro conmutadores está conectado a cuatro antenas, dividiendo así la circunferencia de 16 antenas en cuatro secciones gobernadas por un conmutador cada una. Las señales de control y alimentación provienen de la placa de control y se conectan mediante cables tipo DB9.

\subsubsection{Sistema de control}

Para el control de los conmutadores y alimentación de los mismos, el sistema dispone de un microprocesador basado en la plataforma Arduino Due y una placa de control diseñada para facilitar las conexiones de los conectores DB9.

El microcontrolador recibe las instrucciones programadas en Python y se alimenta a través del puerto USB del ordenador. Las salidas digitales de Arduino, conectadas a la placa de control que ha sido diseñada y fabricada para este trabajo, proporcionan la codificación 
necesaria en cada caso a los conmutadores mediante los cables DB9. Para la alimentación de los conmutadores se ha añadido un pequeño regulador de tensión, de forma que toda la alimentación necesaria en el sistema la proporciona el puerto USB. Por último, la placa incluye unos LEDs que muestran en todo momento la codificación que están recibiendo cada uno de los conmutadores.

\subsection{Algoritmo de visualización de la deformación del tejido}

En la Figura 5 se presenta el esquema del algoritmo computacional utilizado para reconstruir la imagen 3D del cerebro, localizar con precisión las anomalías cerebrales en la cabeza mediante la adquisición de imágenes MRI y visualizar utilizando realidad aumentada (RA) las deformaciones geométricas de los órganos encefálicos en tiempo real. Una vez definidos los modelos anatómicos geométricos, se expone la ubicación de los puntos de control en la superficie cerebral que proporcionarán información en tiempo real de su ubicación. También se expone el método de conexión de dichos puntos con el modelo cerebral completo. Además, se muestra el resultado de los hologramas 3D para visualizar en un sistema de RA unos desplazamientos hipotéticos ocurridos a los tejidos biológicos cerebrales.

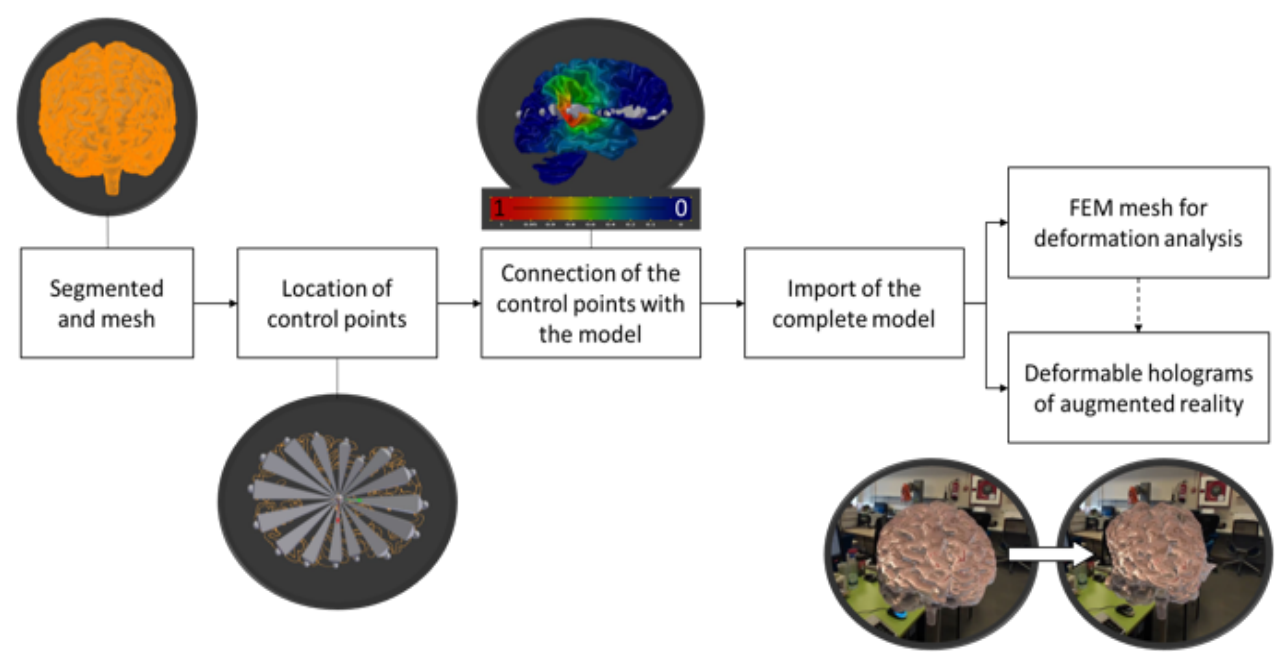

Fig. 5 Esquema para visualización 3D del brain-shift. Fuente: Elaboración propia (2018).

\subsubsection{Segmentación y mallado}

Un paso importante para distinguir órganos, tumores, o cualquier detalle anatómico es la segmentación. Se trata de una de las operaciones más usadas en el procesado digital de imágenes en 3D. Consiste en asignar a cada píxel una clasificación sobre el tipo de tejido que 
representa. Con esta información se pueden definir las superficies de los órganos o lesiones para poder hacer una reconstrucción 3D.

En este trabajo, la segmentación ha sido realizada utilizando un atlas cerebral multimodal basado en imágenes MRI disponible en la página web del Surgical Planning Laboratory (Halle, 2019).

\subsubsection{Ubicación de los puntos de información en la superficie cerebral}

En un trabajo anterior (Bermejo, 2018), se evaluó en un set-up con una geometría sencilla el sistema de medida de distancias usando señales de radiofrecuencia mediante un sistema experimental preliminar. Consistía en las mediciones de una antena para obtener las medidas en el dominio de la frecuencia de distancias entre planos. En el presente trabajo, se presenta una evolución que consiste en utilizar una geometría más compleja, basada en 3D, y similar a la disposición geométrica de los tejidos cerebrales. Se realiza el seguimiento de 16 puntos de control sobre la superficie del tejido phantom.

\section{Resultados}

Se han realizado diversas pruebas del sistema diseñado, utilizando el modelo de cerebro indicado en el apartado 1.2 e introduciendo dentro de la mezcla y en diferentes ubicaciones, un pequeño recipiente de plástico diseñado con impresora 3D que contiene un volumen de $0.5 \mathrm{ml}$ de agua marina. En la Figura 6 se puede ver la solución de la imagen en coordenadas cartesianas de una de las pruebas. En este caso, el elemento extraño se colocó aproximadamente a $200^{\circ}$. Analizando la imagen se puede extraer que el objeto está ubicado a $200^{\circ}$ ya las antenas más cercanas a esta posición reciben un rebote antes que las demás (alrededor de unos $2 \mathrm{~ns}$ ) y con una mayor intensidad. El resto de antenas prácticamente no ven el elemento ya que el material utilizado es muy absorbente.

Para mejorar la calidad de la imagen generada se ha utilizado el algoritmo de Delay-And-Sum (DAS). Esta técnica nos permite detectar variaciones anormales en el dieléctrico a partir de la información de amplitud y fase de la señal recibida. La imagen final se genera a partir del desfase de las señales y la posterior suma de cada uno de los puntos de la imagen a generar. En el algoritmo DAS, la región de interés se divide en una pequeña rejilla o puntos focales. Para cada uno de esos puntos, se calcula la distancia entre los puntos y la antena. A partir de ese valor obtenemos los retardos de las señales y se genera la imagen. En la Figura 7 se representa la imagen de un objeto extraño colocado en $270^{\circ}$ aplicando este algoritmo. 


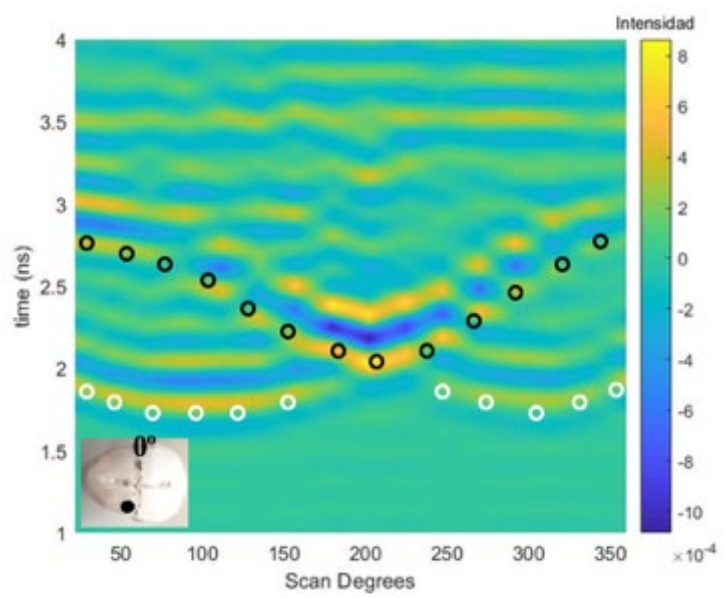

Fig. 6 Resultados de una sesión experimental..Fuente: Elaboración propia (2019).

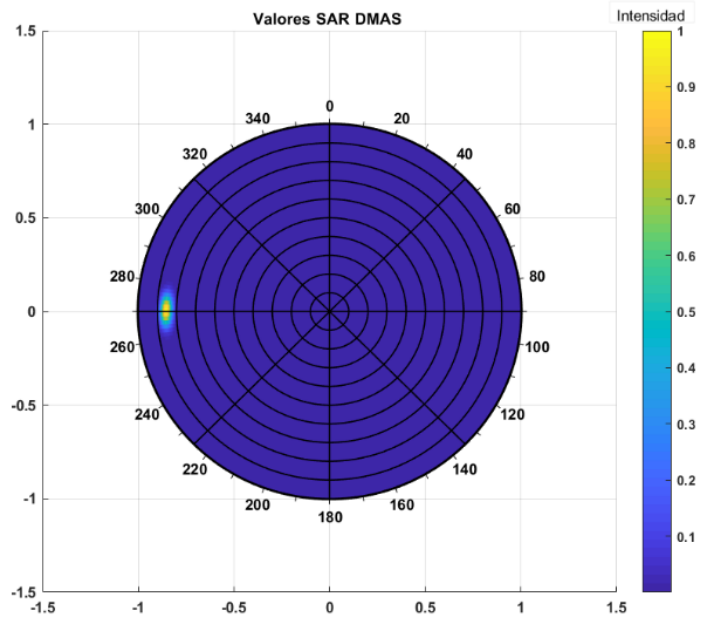

Fig. 7 Resultado en coordenadas circulares con artefacto en 270..Fuente: Elaboración propia (2019).

\section{Conclusiones}

En este trabajo se ha presentado un sistema basado en antenas de banda ancha a frecuencias de microondas para la medida de distancias en deformaciones del tejido cerebral. El sistema está formado por 16 antenas Vivaldi de reducidas dimensiones que trabajan en el rango de 1-5 GHz. Para la conexión de cada antena al analizador vectorial de redes se ha diseñado un 
sistema de conmutación de alta frecuencia controlado por un microcontrolador que, a su vez, está gestionado por un ordenador, donde se procesa la información de cada una de las antenas y se genera la imagen. Para probar el sistema desarrollado se ha utilizado un modelo de cerebro fabricado con materiales de uso general y que presenta propiedades dieléctricas similares al tejido cerebral.

El dispositivo se ha testado en varios supuestos prácticos, utilizando como modelo de tumor agua marina, presentando en todos los casos un buen funcionamiento. El dispositivo desarrollado es capaz de detectar y ubicar artefactos de pequeñas dimensiones, por debajo de $0.5 \mathrm{ml}$ de volumen.

\section{Referencias}

BERMEJO et al. (2018), "Description of the experimental model for the measurement of distance in biological tissues from RF signals," en Spanish Robotics Conference, Valladolid, España.

CHEN, R. E. et al. (2012), "Integrating Retraction Modeling Into an Atlas-Based Framework for Brain Shift Prediction," en IEEE Transactions on Biomedical Engineering, vol. 60, no. 12, pp. 3494-3504.

COMPARETTI M. D. et al. (2011), "Optically tracked multi-robot system for keyhole neurosurgery," en 2011 IEEE International Conference on Robotics and Automation (ICRA), Shanghai, China, pp. 661-666.

DELORENZO, X. et al. (2012), "Volumetric Intraoperative Brain Deformation Compensation: Model Development and Phantom Validation," en IEEE Transactions on Medical Imaging, vol. 31, no. 8, pp. 1607-1619.

HALLE M. et al. (2019) "Multi-modality MRI-based Atlas of the Brain," en SPL 2017 Jan. [en línea]. Disponible en: http://www.spl.harvard.edu/publications/item/view/2037. [Fecha acceso: 12 de febrero de 2019].

LETTEBOER, M. M. J. et al. (2005), "Brain shift estimation in image-guided neurosurgery using 3-D ultrasound," en IEEE Transactions on Biomedical Engineering, vol. 52, no. 2, pp. 268-276.

OLDFIELD, M. J. et al, (2011), "Detailed finite element modelling of deep needle insertions into a soft tissue phantom using a cohesive approach," en Computer Methods in Biomechanics and Biomedical Engineering, vol. 16, no. 5, pp. 530-543. 


\section{INTERFACES CEREBRO-MÁQUINA}




\title{
Control asíncrono de sistemas BCI basados en ERP mediante la detección de potenciales evocados visuales de estado estable provocados por los estímulos periféricos del paradigma oddball
}

\author{
Santamaría-Vázquez, Eduardo; Martínez-Cagigal, Víctor; Gomez-Pilar, Javier y \\ Hornero, Roberto \\ Departamento de Teoría de la Señal y Comunicaciones e Ingeniería Telemática, Universidad de \\ Valladolid, España. Emails: eduardo.santamaria@gib.tel.uva.es, victor.martinez@gib.tel.uva.es, \\ javier.gomez@gib.tel.uva.es,robhor@tel.uva.es.
}

\begin{abstract}
Synchronicity is an inherent feature of brain-computer interface (BCI) spellers based on event related potentials (ERPS). These systems always make a selection, even when users are engaged in another task. This represents a great limitation in real-life applications, such as wheelchair control or web browsers, in which an asynchronous control should be a key feature. The aim of this study is to design, develop and test a novel algorithm to discriminate whether the user wants to select a command or is not attending the stimuli. In order to achieve such asynchronous control, our method detects the steady-state visual evoked potentials provoked by nontarget stimuli of ERP-based spellers. The proposed method was validated with offline data from 5 healthy subjects, achieving an average accuracy of 99.7\%. Our approach is independent of the ERP classification stage, which reduces inter-session variability. Furthermore, to the best of our knowledge, it is the first algorithm for asynchronous control that does not need to extend the duration of the calibration sessions.
\end{abstract}

Keywords: Brain-computer interfaces, event-related potentials, asynchrony, control-state detection, steady-state visual evoked potentials, P300.

\section{Resumen}

Los sistemas Brain-computer interface (BCI) basados en potenciales relacionados con eventos (ERPS) son sistemas intrínsecamente síncronos que realizan selecciones de manera constante, incluso cuando el usuario no está prestando atención a los estímulos. Esto representa una gran limitación en aplicaciones reales, donde se requiere un control asíncrono. El objetivo de este estudio es diseñar, desarrollar y evaluar un novedoso método de asincronía que discrimine si el usuario quiere seleccionar un comando o está realizando otra tarea. Para alcanzar este objetivo, nuestro método detecta 
los potenciales evocados visuales de estado estable provocados por los estímulos no objetivo del paradigma oddball. Este método ha sido probado en 5 sujetos sanos, alcanzando una precisión del 99.7\% en la detección del estado de control. Este algoritmo es independiente del método de clasificación de los ERPs, reduciendo la variabilidad inter-sesión. Además, hasta donde tenemos conocimiento, es el primer método de asincronía que no necesita extender las sesiones de calibración del sistema.

Palabras clave: Interfaces cerebro-ordenador, potenciales relacionados con eventos, detección del estado de control, asincronía, potenciales evocados visuales de estado estable, P300.

\section{Introducción}

Los sistemas Brain-computer interface $(\mathrm{BCI})$ permiten la comunicación directa entre el cerebro y un dispositivo externo, identificando las intenciones del usuario a partir de su actividad neuronal. En la actualidad, la principal aplicación de los sistemas BCI es la mejora de la independencia y la calidad de vida de personas con grave discapacidad, proporcionando una alternativa accesible a los canales de comunicación habituales, que requieren el control de músculos y nervios periféricos (Wolpaw et al., 2002). En la práctica, estos sistemas emplean la eletroencefalografía (EEG) para registrar la señal eléctrica del cerebro ya que es una técnica portable, no invasiva y de bajo coste en comparación con otras disponibles, como la magnetoencefalografía (MEG), la electrocorticografía (ECoG), o la espectroscopia de infrarrojo cercano funcional (fNIRS) (Wolpaw \& Wolpaw, 2012).

La señal de EEG es la superposición de componentes cíclicas, denominadas ritmos, y transitorias que reflejan la actividad subyacente del cerebro. Particularmente, los potenciales relacionados con eventos (event-related pontentials, ERPs) son la respuesta natural del cerebro ante diferentes tipos de eventos. Los ERPs provocados por un estímulo visual (e.g. una iluminación repentina) se conocen como ERPs visuales (Luck, 2014). Farwell y Donchin (Farwell \& Donchin, 1988) propusieron por primera vez un sistema BCI que utilizaba los ERPs visuales para detectar las intenciones de los usuarios mediante el paradigma oddball. Este paradigma presenta una matriz de comandos al usuario, cuyas filas y columnas se iluminan de manera aleatoria. La iluminación de todas las filas y columnas de la matriz se denomina secuencia. Durante este proceso, el usuario debe mirar fijamente el comando que desea seleccionar, emitiendo un ERP cuando este se ilumina. El sistema analiza las épocas de señal inmediatamente posteriores a cada estimulación, determinando la fila y columna que tienen mayor probabilidad de contener un ERP. Una vez detectada la fila y la columna, se selecciona el comando correspondiente y se vuelve a empezar el 
proceso. Para mejorar la precisión del sistema, es habitual que se realicen varias secuencias de estimulación, aumentando la confianza en la selección.

Numerosos estudios han mejorado la precisión y la velocidad de selección de comandos en los sistemas BCI basados en ERPs (Aydin et al., 2018; Rezeika et al., 2018; Schettini et al., 2014). Sin embargo, aún existen grandes limitaciones que impiden su uso fuera de un entorno controlado de laboratorio. En concreto, los sistemas BCI basados en ERPs son sistemas síncronos, lo que implica que siempre se selecciona un comando en cada iteración del algoritmo, incluso aunque el usuario no esté prestando atención a los estímulos. Esto supone una gran limitación para la mayoría de aplicaciones reales de estos sistemas BCI, como el control de una silla de ruedas o la navegación web, donde detectar si el usuario está controlando la aplicación es un requisito fundamental para su funcionamiento (Aloise et al., 2011; Zhang et al., 2008).

Recientemente, varios estudios han intentado abordar este problema. En general, los algoritmos propuestos hasta el momento definen un umbral sobre diferentes medidas derivadas de la salida de la etapa de clasificación de los ERPs (Aydin et al., 2018; Martínez-Cagigal et al., 2017; Zhang et al., 2008). Sin embargo, este enfoque presenta varios inconvenientes. En primer lugar, estos algoritmos presentan una alta variabilidad inter-sesión (Schettini et al., 2014). La amplitud y latencia de los ERPs de una misma persona es variable en el tiempo, provocando que la precisión asociada al umbral establecido en la etapa de calibración se vea reducida en sesiones posteriores. Además, estos algoritmos necesitan extender las sesiones de calibración del clasificador para adquirir datos del usuario mientras no está controlando la aplicación, lo que reduce su usabilidad en un entorno real. El desarrollo de nuevos algoritmos independientes de la etapa de clasificación de los ERPs podría ayudar a superar estas limitaciones. Pinegger et al. (Pinegger et al., 2015) desarrollaron una primera aproximación basada en la hipótesis de que la frecuencia de estimulación a la que se iluminan las filas y columnas de la matriz de comandos estaría presente en el EEG del usuario, alcanzando un $79.5 \%$ de precisión. A pesar de la novedad de este enfoque, el rendimiento alcanzado no permite un control asíncrono satisfactorio de la aplicación.

El objetivo de este estudio es diseñar, desarrollar y evaluar un novedoso método para la detección del estado de control del usuario en sistemas BCI basados en ERPs que permita superar las limitaciones anteriores. Nuestra hipótesis de partida es que las estimulaciones periféricas de la matriz de comandos provocan un potencial evocado de estado estable (steady-state visual evoked potential, SSVEP) en el usuario cuando está controlando el sistema. Los SSVEPs son formas de onda similares a sinusoides que aparecen en el EEG cuando se recibe un estímulo visual repetitivo a una frecuencia constante (Luck, 2014). El método diseñado es independiente de la etapa de clasificación de los ERPs. Además, hasta 
donde tenemos conocimiento, es el primer método de asincronía que no necesita aumentar la duración de las sesiones de calibración.

\section{Métodos}

\subsection{Adquisición de la señal y sujetos}

La señal de EEG fue registrada con 8 electrodos situados en las posiciones $\mathrm{Fz}, \mathrm{Cz}, \mathrm{Pz}, \mathrm{P}$, P4, PO7, PO8 y Oz de acuerdo con el sistema internacional 10-20 extendido. Esta configuración es habitual en los sistemas BCI basados en ERPs (Aloise et al., 2011). Se ha usado un equipo g.USBamp para la amplificación y conversión de la señal de EEG al dominio digital, utilizando una frecuencia de muestreo de $256 \mathrm{~Hz}$. La plataforma MEDUSA $^{\circledR}$ ha sido utilizada para registrar y guardar los datos, procesar la señal de EEG y presentar las matrices de estimulación a los participantes (Santamaría-Vázquez et al., 2018).

Cinco sujetos sanos (media $25.8 \pm 1.09$ años) han participado en los experimentos. El protocolo de experimentación ha sido aprobado por el comité ético correspondiente y todos los participantes han dado su consentimiento de participación informado.

\subsection{Método propuesto}

El método propuesto, denominado Oddball Steady Response Detection (OSRD), es un nuevo algoritmo para detectar el estado de control del usuario en sistemas BCI basados en ERPs. El algoritmo debe diferenciar entre dos estados de control: (i) el usuario está atendiendo a los estímulos y quiere seleccionar un comando, y (ii) el usuario no está atendiendo a los estímulos y no quiere seleccionar ningún comando. OSRD recibe como entrada la señal EEG cruda correspondiente a la selección de un comando. Una vez procesada la señal, el algoritmo proporciona una salida binaria $y \in\{0,1\}$ en función del estado de control detectado. A continuación, se detallan las diferentes etapas del método OSRD.

\subsubsection{Pre-procesado de la señal}

El objetivo de esta etapa es incrementar la relación señal a ruido (SNR). Se aplica un filtro paso banda entre $\left[f_{s t}-b w_{1} / 2, f_{s t}+b w_{1} / 2\right]$, donde $f_{s t}$ es la frecuencia de estimulación del paradigma (inversa del tiempo entre dos iluminaciones consecutivas) y $b w_{1}$ es heurísticamente seleccionado como $1 \mathrm{~Hz}$. Posteriormente, se aplica un filtro espacial 
referencia media común (common average reference, CAR) para eliminar las componentes de ruido comunes a todos los canales del EEG (Martínez-Cagigal et al., 2019).

\subsubsection{Extracción de características}

OSRD extrae dos características para diferenciar entre los dos estados de control del usuario. La primera está basada en el método de análisis de correlación canónica (canonical correlation analysis, CCA) (Krzanowski, 2000). Este análisis estadístico multivariante permite encontrar correlaciones subyacentes entre dos conjuntos de datos multidimensionales, siendo utilizado habitualmente para la detección de SSVEPs (Zhang et al., 2013). CCA encuentra la combinación lineal óptima de cada conjunto de datos (i.e. $\boldsymbol{X}, \boldsymbol{Y})$ para maximizar la correlación entre ambos. En este caso, $\boldsymbol{X}$ es la señal de EEG correspondiente a un trial, con dimensiones $N \times C$, donde $N$ es el númeo de muestras y $C$ el número de canales utilizados. Por otra parte, $\boldsymbol{Y}$ es la señal de referencia, que es un seno de frecuencia $f_{s t}$ y dimensiones $N \times 1$. Consecuentemente, la primera característica es el coeficiente de correlación máximo entre $\boldsymbol{X}$ e $\boldsymbol{Y}$ :

$$
c_{1}=C C A(\boldsymbol{X}, \boldsymbol{Y})
$$

La segunda característica $\left(c_{2}\right)$, se deriva directamente de la estimación del espectro del EEG. Con el objetivo de aumentar la resolución espectral, se concatenan todos los canales del EEG en un único vector de dimensiones $N \cdot C \times 1$. Posteriormente, se calcula la densidad espectral de potencia (power spectral density, PSD) de este vector mediante el método de Welch. La segunda característica es la diferencia entre el valor medio de la PSD en un rango estrecho alrededor de $f_{s t}$, y el valor medio en un rango más amplio:

$$
c_{2}=\frac{1}{b w_{2}} \int_{f_{s t}+b w_{2} / 2}^{f_{s t}+b w_{2} / 2} S(f) d f-\frac{1}{b w_{1}} \int_{f_{s t}+b w_{1} / 2}^{f_{s t}+b w_{1} / 2} S(f) d f,
$$

donde $S(f)$ representa la PSD, y $b w_{1}$ y $b w_{2}$ fueron fijados a 2 y $0.1 \mathrm{~Hz}$ respectivamente. Estos valores han sido escogidos heurísticamente para considerar suficientes muestras dentro de cada una de las bandas de manera que aumente la robustez del método frente al ruido.

\subsubsection{Observaciones sintéticas}

Los sistemas BCI basados en ERPs deben ser recalibrados debido a la alta variabilidad inter-sesión de estas formas de onda (Schettini et al., 2014). De hecho, para asegurar un rendimiento adecuado, es recomendable que el usuario seleccione varios comandos de entrenamiento antes de utilizar el sistema. Este procedimiento requiere una gran cantidad tiempo, haciendo que el sistema sea poco práctico en la vida real. Además, todos los métodos de asincronía definidos hasta el momento requieren aumentar la duración de estas 
sesiones de calibración para adquirir señales de los usuarios mientras no están controlando la aplicación. OSRD supera esta limitación mediante la creación de observaciones sintéticas que simulan a aquellas que se obtendrían mientras el usuario no está atendiendo a los estímulos. Asumiendo que la PSD del EEG mantiene sus características alrededor de la frecuencia $f_{s t}$, se puede simular una observación mediante el desplazamiento de la frecuencia $f_{s t}^{\prime}=f_{s t}+f_{0}$, donde $f_{0}$ es fijada a $0.5 \mathrm{~Hz}$ de manera heurística. Posteriormente, este valor se usa en las ecuaciones 1 y 2 para realizar los cálculos. Este procedimiento se basa en la hipótesis de que las características de la señal de EEG cuando el usuario está atendiendo a los estímulos en la frecuencia $f^{\prime}{ }_{s t}$ son similares a las obtenidas en la frecuencia $f_{s t}$ cuando el usuario no está controlando el sistema.

\subsubsection{Clasificación de características}

La última etapa del algoritmo determina el estado de control del usuario a partir de las características $c_{1}$ y $c_{2}$. OSRD asigna $y=1$ si se detecta que el usuario estaba atendiendo a los estímulos de la matriz de comandos, o $y=0$ en caso contrario. El método utilizado para la clasificación de características es el análisis discriminante lineal (linear discriminant analysis, LDA), debido a su amplio uso en sistemas BCI (Martínez-Cagigal et al., 2019; Pinegger et al., 2015; Wolpaw \& Wolpaw, 2012).

\subsection{Análisis experimental}

Se realizaron dos análisis independientes con el objetivo de comprobar la eficacia del método de asincronía propuesto. Los sujetos realizaron dos sesiones idénticas, en cada una de las cuales se registraron 30 selecciones mientras los usuarios estaban atendiendo a los estímulos, y 30 selecciones mientras los usuarios estaban visualizando un video. Por tanto, el conjunto de datos total estaba formado por 120 selecciones, 60 de cada tipo. Cada una de las selecciones estaba formada por 15 secuencias de estimulación. Durante estos registros, se utilizó la matriz de comandos representada en la figura 1. La frecuencia de estimulación durante las pruebas se fijó en $f_{s t}=5.71 \mathrm{~Hz}$ (Pinegger et al., 2015).

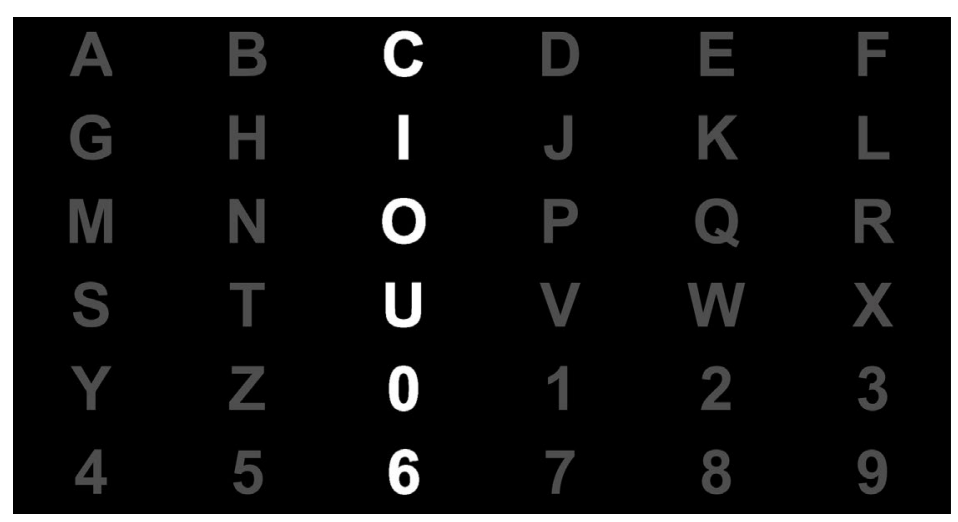

Fig. 1. Matriz de comandos utilizada durante los experimentos con la tercera columna iluminada. 
En el primer análisis, se aplicó a cada participante por separado el procedimiento leaveone-out (LOO) en el conjunto de 120 selecciones. En cada iteración del algoritmo, la fase de entrenamiento del clasificador usó la señal completa de cada selección (15 secuencias). Sin embargo, en la fase de evaluación se consideró la señal correspondiente a 1, 5, 10 o 15 secuencias, con el objetico de analizar la precisión del sistema en función de este parámetro.

En el segundo análisis el procedimiento de evaluación se modificó de la siguiente manera: en la fase de entrenamiento solamente se consideraron las 60 selecciones realizadas por cada usuario mientras atendía a los estímulos, creando otras 60 observaciones sintéticas en estado de no-control a partir de ellas. Sin embargo, en la fase de evaluación del procedimiento LOO, se utilizaron selecciones en estado de no-control reales. El objetivo de este análisis era determinar si las observaciones sintéticas permiten un correcto funcionamiento del algoritmo.
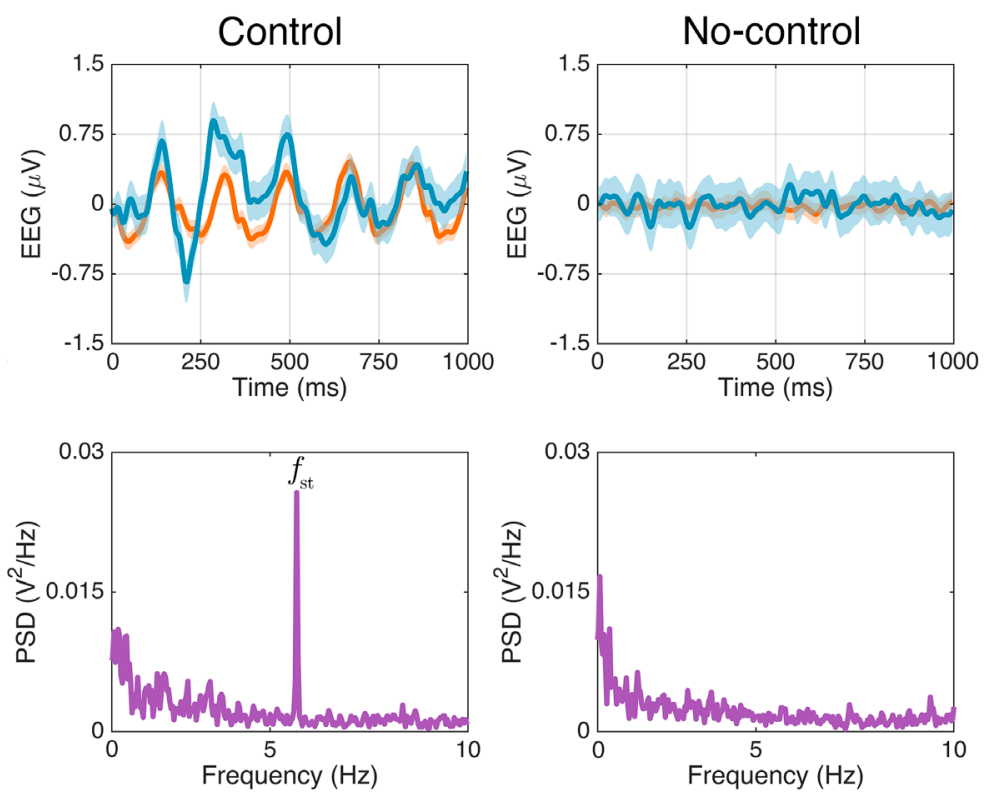

Fig. 2. Representación temporal y espectral del EEG en los dos estados de control para el usuario U05 en el canal Cz. En la parte de superior se muestran las épocas de señal promediadas para estímulos objetivo (azul) y periféricos (naranja). La parte sombreada representa el intervalo de confianza al 95\%. En la parte inferior se muestra la PSD de la señal correspondiente. Como se puede observar, en la PSD correspondiente al estado de control se refleja un pico a la frecuencia de estimulación del paradigma. 


\section{Resultados}

La figura 2 muestra la representación temporal y frecuencial de las señales en estado de control y en estado de no-control del sujeto U05, en el canal Cz. En las dos subfiguras superiores, la línea azul representa la media de las épocas de señal correspondientes a los estímulos objetivo, mientras que la línea naranja representa la media de las épocas correspondientes a estímulos no-objetivo. El área sombreada representa el intervalo de confianza al 95\%. En la parte de debajo de la figura se representa la PSD de la señal correspondiente a todas las épocas de señal.

Los resultados de clasificación de los dos análisis realizados se muestran en la tabla 1. En ella se observan las precisiones alcanzadas por OSRD en la fase de test de LOO utilizando observaciones de no-control reales (columna R) y sintéticas (columna S). Las precisiones están desglosadas por número de secuencias.

\section{Discusión}

En este estudio se ha evaluado un nuevo método de asincronía para sistemas BCI basados en ERPs. Este método se basa en la detección de los SSVEPs provocados por las estimulaciones periféricas del paradigma oddball. En la figura 2 podemos observar como la representación temporal del EEG cuando el usuario está controlando el sistema muestra el SSVEP tanto en las épocas de señal correspondientes a los estímulos objetivo (iluminaciones del comando que el usuario quiere seleccionar) como en las correspondientes a los estímulos no objetivo (iluminaciones del resto de comandos). Por el contrario, sólo las épocas de señal correspondientes a las iluminaciones del comando deseado muestran el ERP del usuario, incluyendo el potencial evocado P300. Asimismo, la

Tabla 1. Resultados de los análisis

\begin{tabular}{|c|c|c|c|c|c|c|c|c|}
\hline & \multicolumn{8}{|c|}{ No. Secuencias } \\
\hline & \multicolumn{2}{|c|}{1} & \multicolumn{2}{|c|}{5} & \multicolumn{2}{|c|}{10} & \multicolumn{2}{|c|}{15} \\
\hline & $\mathbf{R}$ & $\mathbf{S}$ & $\mathbf{R}$ & $\mathbf{S}$ & $\mathbf{R}$ & $\mathbf{S}$ & $\mathbf{R}$ & $\mathbf{S}$ \\
\hline U01 & 63.3 & 64.2 & 94.2 & 95.0 & 97.5 & 97.5 & 100 & 100 \\
\hline U02 & 65.8 & 64.2 & 95.8 & 95.0 & 96.7 & 96.7 & 98.3 & 98.3 \\
\hline U03 & 63.3 & 62.5 & 97.5 & 97.5 & 100 & 100 & 100 & 100 \\
\hline U04 & 60.0 & 56.7 & 94.2 & 95.0 & 99.2 & 98.3 & 100 & 100 \\
\hline U05 & 73.3 & 72.5 & 96.7 & 96.7 & 99.2 & 99.2 & 100 & 100 \\
\hline Media & 65.1 & 64.0 & 95.7 & 95.8 & 98.5 & 98.3 & 99.7 & 99.7 \\
\hline STD & 4.5 & 5.1 & 1.3 & 1.1 & 1.2 & 1.7 & 0.7 & 0.7 \\
\hline
\end{tabular}


representación frecuencial de la señal muestra claramente un pico a la frecuencia de estimulación de la matriz, correspondiente con el SSVEP generado. Por el contrario, ninguno de estos fenómenos se aprecia cuando el usuario está realizando otra tarea.

En la tabla 1 se muestran los resultados de clasificación. No se han encontrado diferencias significativas, independientemente del número de secuencias considerado, entre las precisiones alcanzadas cuando se entrena el algoritmo con selecciones no-control reales o sintéticas (test de rangos con signo de Wilcoxon, $p$-valor $>0.05$ ). Estos resultados sugieren que la duración de las sesiones de calibración podría reducirse a la mitad, registrando únicamente selecciones mientras el usuario está atendiendo a los estímulos, sin que el rendimiento en la detección del estado de control del usuario se vea comprometido. Asimismo, se aprecia cómo la precisión crece a medida que se consideran más secuencias de estimulación, alcanzando un máximo del $99.7 \%$ para 15 secuencias.

Aunque existen varios estudios que han tratado previamente el problema de la asincronía en sistemas BCI basados en ERPs, únicamente el estudio de Pinegger et al. (Pinegger et al., 2015) permite una comparación directa con este trabajo por ser el único que proporciona la precisión en la detección del estado de control del usuario de manera aislada. En este artículo se presentan dos métodos de asincronía diferentes, evaluados en 21 sujetos de control y utilizando 15 secuencias de estimulación. El primer método utiliza la salida de la etapa de clasificación de los ERPs, detectando que el usuario estaba atendiendo a los estímulos cuando la probabilidad de clasificación superaba un determinado umbral, alcanzando una precisión del 94.2\%. El segundo método, al igual que el presente estudio, detecta los SSVEPs provocados por el paradigma oddball independientemente de la etapa de clasificación de los ERPs, alcanzando una precisión del 79.5\%. Por último, la hibridación de los dos métodos alcanza un 95.5\% de precisión. En comparación, OSRD alcanza una precisión del $99.7 \%$ con el mismo número de secuencias de estimulación, superando a los tres métodos anteriores. Además, es el único que no necesita aumentar la duración de las sesiones de calibración gracias al cálculo de observaciones no-control sintéticas.

A pesar de los resultados positivos alcanzados en este estudio, se pueden señalar varias limitaciones. En primer lugar, el método ha sido evaluado con 5 sujetos de control. Un mayor número de sujetos permitiría asegurar la generalización de los resultados. Asimismo, el sistema no se ha probado con sujetos con grave discapacidad, la población que presumiblemente se beneficiaría del método. La inclusión de nuevas medidas complementarias basadas en análisis estadístico podría mejorar los resultados de clasificación, y deberían ser estudiadas en el futuro (Martínez-Cagigal et al., 2019). En este estudio únicamente se han analizado los resultados de clasificación a la hora de detectar el estado de control del usuario. Sin embargo, en el futuro sería de gran utilidad analizar 
también la precisión en la selección de comandos de un sistema que utilice OSRD para la monitorización de la atención del usuario, así como probar el método en sesiones online.

\section{Conclusiones}

Este estudio presenta un novedoso método para determinar el estado de control del usuario en sistemas BCI basados en ERPs mediante la detección de los SSVEPs provocados por el paradigma oddball. El método propuesto ha sido evaluado en 5 sujetos sanos, alcanzando una precisión media con 15 secuencias de estimulación del 99.7\%, superando a otros métodos actuales. Además, éste presenta dos ventajas respecto a otros enfoques anteriores: (i) es independiente de la etapa de clasificación de los ERPs para la selección de comandos, lo que reduce la variabilidad inter-sesión que afecta a estas formas de onda; y (ii) es, hasta donde tenemos conocimiento, el primer método que no necesita registrar la señal de EEG mientras el usuario no está controlando el sistema BCI, reduciendo drásticamente el tiempo de calibración necesario.

\section{Agradecimientos}

Este estudio ha sido financiado por el proyecto DPI2017-84280-R del Ministerio de Ciencia, Innovación y Universidades y FEDER y el proyecto "Análisis y correlación entre el genoma completo y la actividad cerebral para la ayuda en el diagnóstico de la enfermedad de Alzheimer" (Programa Operativo de Cooperación Transfronteriza EspañaPortugal, POCTEP, 2014-2020) de la Comisión Europea y FEDER. Eduardo SantamaríaVázquez es beneficiario de una ayuda de Personal Investigador en Formación (PIF) financiada por la Consejería de Educación de la Junta de Castilla y León y el Fondo Social Europeo. Víctor Martínez-Cagigal es beneficiario de una ayuda PIF-UVa de la Universidad de Valladolid.

\section{Referencias}

Aloise, F., Schettini, F., Aricò, P., Leotta, F., Salinari, S., Mattia, D., Babiloni, F., Cincotti, F., 2011. P300-based brain-computer interface for environmental control: An asynchronous approach. J. Neural Eng. 8.

Aydin, E.A., Bay, O.F., Guler, I., 2018. P300-Based Asynchronous Brain Computer Interface for Environmental Control System. IEEE J. Biomed. Heal. Informatics 22, 653-663.

Farwell, L.A., Donchin, E., 1988. Talking off the top of your head: toward a mental 
prosthesis utilizing event-related brain potentials. Electroencephalogr. Clin. Neurophysiol. 70, 510-523.

Krzanowski, W., 2000. Principles of multivariate analysis. OUP Oxford.

Luck, S.J., 2014. An introduction to the event-related potential technique. MIT press.

Martínez-Cagigal, V., Gomez-Pilar, J., Álvarez, D., Hornero, R., 2017. An Asynchronous P300-Based Brain-Computer Interface Web Browser for Severely Disabled People. IEEE Trans. Neural Syst. Rehabil. Eng. 25, 1332-1342.

Martínez-Cagigal, V., Santamaría-Vázquez, E., Gomez-Pilar, J., Hornero, R., 2019. Towards an accessible use of smartphone-based social networks through braincomputer interfaces. Expert Syst. Appl. 120, 155-166.

Pinegger, A., Faller, J., Halder, S., Wriessnegger, S.C., Müller-Putz, G.R., 2015. Control or non-control state: that is the question! An asynchronous visual P300-based BCI approach. J. Neural Eng. 12, 014001.

Rezeika, A., Benda, M., Stawicki, P., Gembler, F., Saboor, A., Volosyak, I., 2018. Braincomputer interface spellers: A review. Brain Sci. 8.

Santamaría-Vázquez, E., Martínez-Cagigal, V., Hornero, R., 2018. MEDUSA: Una Nueva Herramienta Para El Desarrollo De Sistemas Brain-Computer Interface Basada en Python. Cognitive Area Networks 5, 87-92

Schettini, F., Aloise, F., Aricò, P., Salinari, S., Mattia, D., Cincotti, F., 2014. Selfcalibration algorithm in an asynchronous $\mathrm{P} 300$-based brain-computer interface. J. Neural Eng. 11.

Wolpaw, J., Wolpaw, E.W., 2012. Brain-computer interfaces: principles and practice. OUP USA.

Wolpaw, J.R., Birbaumer, N., McFarland, D.J., Pfurtscheller, G., Vaughan, T.M., 2002. Brain Computer Interfaces for communication and control. Clin. Neurophysiol. 4, 767-791.

Zhang, H., Guan, C., Wang, C., 2008. Asynchronous P300-based brain-computer interfaces: a computational approach with statistical models. IEEE Trans. Biomed. Eng. 55, 1754-63.

Zhang, Y., Zhou, G., Jin, J., Wang, X., Cichocki, A., 2013. Frequency Recognition in SSVEP-based BCI using Multiset Canonical Correlation Analysis. Int. J. Neural Syst. 24. 


\title{
Estudio preliminar de la detección de cambios de velocidad de la marcha a partir de señales EEG
}

\section{Quiles Zamora, Vicente; Iáñez, Eduardo; Ortiz, Mario y Azorín, José M.}

Brain-Machine Interface Systems Lab, Universidad Miguel Hernández de Elche, Avda. de la Universidad S/N, 03202 Elche, España, vquiles@umh.es, eianez@umh.es, mortiz@umh.es, jm.azorin@umh.es.

\begin{abstract}
The main objective of this work is analysing electroencephalographic (EEG) signals to get a mark to link brain information with characteristic indicators of the walk speed. This starts with developing a Brain-Machine Interface (BMI) and designing a validation protocol to allow to conclude an accuracy rate to assure reliable control of the BMI. It pretends to create a robust arquitecture that will be improvable in the future being able to provide a feedback with the pacient in real time.
\end{abstract}

Keywords: BMI, EEG, CWT, speed, walk.

\section{Resumen}

El objetivo de este estudio es analizar las señales electroencefalográficas (EEG) para obtener un indicador que relacione la información cerebral con marcadores de velocidad en la marcha. El planteamiento inicial pasa por desarrollar una interfaz cerebro-máquina (BMI) y el diseño de un protocolo de validación que permita concluir una tasa de acierto que asegure un correcto control de la BMI por parte de un paciente. Se pretende así crear una arquitectura robusta que asiente las bases para un modelo futuro en el que la interfaz controle en tiempo real la marcha del paciente mediante un exoesqueleto.

Palabras clave: EEG, BMI, marcha, velocidad. 


\section{Introducción}

En la última década se ha producido un gran avance en los sistemas basados en la interacción hombre-máquina. Hasta, hace unos pocos años éstos estaban limitados a una actuación física por parte del usuario. Sin embargo el impulso de otro tipo de interfaces, como aquellas basadas en reconocimiento por voz, seguimiento ocular, señales EMG o señales cerebrales, ofrece nuevas posibilidades de interacción, especialmente para personas con limitaciones motoras en sus miembros inferiores y/o superiores. Estos avances junto al de mecanismos a la ayuda motora cada vez más sofisticados como los exoesqueletos, permiten crear nuevas terapias de rehabilitación que pueden ser beneficiosas en etapas iniciales de lesiones motoras (Murie et al., 2010). Algunos de los beneficios de la utilización de este tipo de sistemas en la rehabilitación resultan en un aumento de la vascularización, el equilibrio o la estabilidad, entre otros (Gorgey, 2018).

El análisis de las señales electroencefalográficas (EEG) es un campo de investigación novedoso, siendo su aplicación en interfaces cerebro-máquina (Brain-Machine Interfaces, BMIs, en inglés) una herramienta interesante para la ayuda a la marcha en terapias de rehabilitación (Dobkin, 2007). La posibilidad de detectar la intención del movimiento antes de que éste ocurra ofrece grandes posibilidades para mejorar el proceso de rehabilitación (Pfurtscheller, 1999). Algunos estudios plantean el uso de potenciales sensoriomotores para la detección de la intención de inicio, fin de marcha o cambios de dirección (Hortal et al., 2016). Estos potenciales vienen relacionados con fluctuaciones en las bandas de frecuencias alpha y beta momentos antes de la actividad motora (Toffanin et al., 2007).

Sin embargo, las terapias de rehabilitación parten de la premisa que debe existir una retroalimentación entre las actuaciones del usuario y el sistema, lo que deriva en un análisis de estos potenciales en tiempo real, con la finalidad de poder suministrar esta información a los sistemas de ayuda a la marcha. La interfaz que se propone en este trabajo pretende ser un primer acercamiento al desarrollo de un sistema online que permita detectar la intención de cambiar de velocidad de la marcha a partir del análisis de las señales EEG y regule el control de velocidad de un exoesqueleto.

En este trabajo planteamos un modelo que discierne entre dos clases, velocidad continuada e intención de incrementar velocidad. Para ello realizaremos un estudio que determine el cambio de aceleraciones, para que nuestro modelo sea capaz de entrenar con los datos generados por los equipos de adquisición no invasivos de EEG los dos estados descritos. 


\section{Materiales y métodos}

En este apartado se describe el material utilizado, así como el protocolo planteado en las pruebas. También detallamos los métodos de procesamiento, filtrado de ruido y extracción de características, para las señales cerebrales y de movimiento. Por último describimos el modelo de clasificación planteado y el método de validación.

\subsection{Configuración experimental}

Para el registro de señales EEGs se ha utilizado el equipo amplificador de 24 bits actiCHamp de BrainProducts en combinación con un gorro de electrodos activos de 32 canales incluida la referencia. La configuración del equipo es parametrizable a través del programa PyCorder, el cual permite medir las impedancias de los electrodos, facilitando una correcta puesta a punto del mismo. Además el programa se encarga de desreferenciar las señales, implementar un paso banda de $0.1-100 \mathrm{~Hz}$ y aplicar un filtro Notch a $50 \mathrm{~Hz}$ para evitar interferencias de la red de alimentación.

La disposición de los electrodos empleada para registrar las señales EEG, siguiendo el sistema internacional 10/10, es la siguiente: Fz, FC5, FC3, FC1, FCz, FC2, FC4, FC6, C5, $\mathrm{C} 3, \mathrm{C} 1, \mathrm{Cz}, \mathrm{C} 2, \mathrm{C} 4, \mathrm{C} 6, \mathrm{CP} 5, \mathrm{CP} 3, \mathrm{CP} 1, \mathrm{CP}$, CP2, CP4, CP6, P3, P1, Pz, P2, P4, FO7, FO3, Fpz, FO4, FO6. La señal esta doblemente referenciada al lóbulo izquierdo mediante un electrodo de oreja y a Fz (Jiang et al., 2015). La captación de la señal se realiza vía la comunicación entre el Pycorder y Matlab, donde realizamos el procesamiento y análisis de nuestros datos de forma offline.

Para el registro de la marcha el equipo utilizado es el tech-MCS V3 de Technaid, el cual permite el registro de las variables de movimiento del sujeto a partir de 7 unidades inerciales (IMUs) que nos proporcionan valores a una frecuencia de muestreo de $30 \mathrm{~Hz}$ para la aceleración en formato triaxial. Los sensores se colocan en las siguientes posiciones: lumbar, muslo derecho, espinilla derecha, pie derecho, muslo izquierdo, espinilla izquierda y pie derecho. El equipo procesa el ruido mediante un filtro Kalman según especifica TECHNAID. Por cada una de las IMU se extraen tres características: 'AccX', 'AccY' y 'AccZ', las cuales son procesadas posteriormente. Queda excluida la IMU de la lumbar por desvirtuar la forma de la señal promedio.

El equipo se coloca sobre un carrito móvil de dos niveles en el cual colocamos el portátil que procesa los datos y el amplificador (Fig1). 


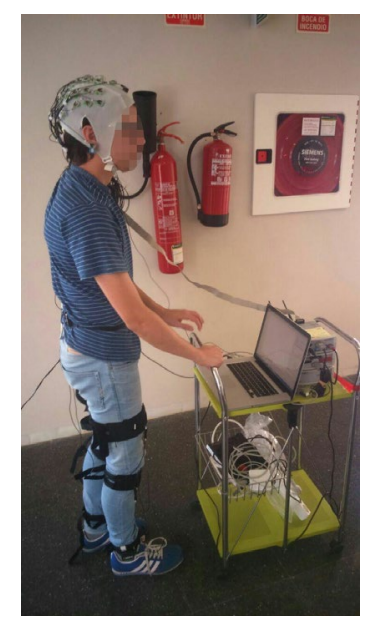

Fig1. Montaje experimental para la realización de la prueba

\subsection{Procedimiento experimental}

Se han realizado dos experimentos distintos con protocolos diferentes, el primero más centrado en el análisis de la marcha y el segundo en el estudio de las señales EEG para la validación de la interfaz BMI, que debe predecir la intención del sujeto de cambiar de velocidad.

Gran parte del éxito en la detección de la intención del cambio de velocidad pasa por localizar correctamente el instante concreto en el cual se realiza el cambio de velocidad. Para la evaluación de la detección de dicho instante de cambio participaron cuatro primeros sujetos con edades comprendidas entre 18 y 24 años, todos diestros. El protocolo seguido en esta evaluación fue: el usuario comienza la marcha a una velocidad relativamente constante, con paso normal, y, aproximadamente cuatro segundos después se le pide que acelere lo más rápidamente posible y mantenga la velocidad otros tres segundos marcando el momento del cambio.

Para evaluar la BMI que proponemos se realizaron pruebas con tres sujetos con edades comprendidas entre 22 y 24 años, uno zurdo y dos diestros. Cada sujeto realizó 40 repeticiones del protocolo siguiente: el usuario permanece en reposo y tras $5 \mathrm{~s}$ se le ordena que inicie la marcha a una velocidad constante y paso normal, y transcurridos aproximadamente 5-6s decide voluntariamente cambiar de velocidad manteniéndola de forma constante durante $3.4 \mathrm{~s}$ más aproximadamente (Fig2). Cada registro realizado consiste en un recorrido de 15 metros aproximadamente. 
Como normas para la realización de las pruebas se les especifica que deben intentar minimizar parpadeos, movimientos mandibulares o posiciones gesticulares anómalas, así como realizar un paso firme y evitar zancadas bruscas, sobre todo en el tramo de marcha con mayor velocidad. En algunos casos se han descartado registros, por fallos en el protocolo, desprendimiento del equipo de captación de movimiento, distracciones por parte del sujeto o incumplimiento de alguna de las normas.

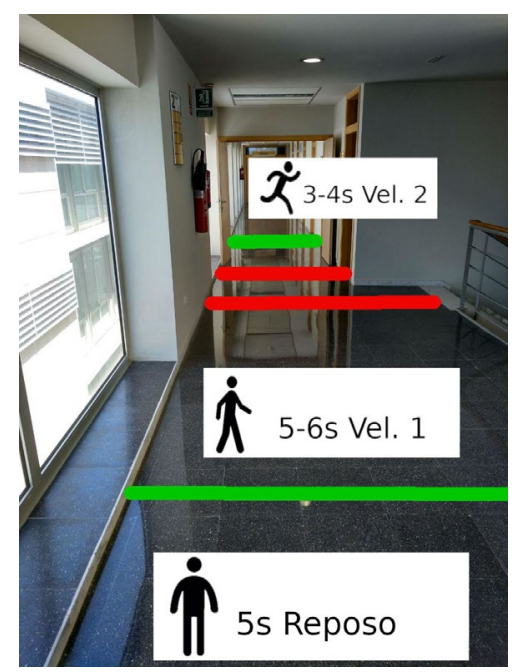

Fig2. El sujeto espera en reposo hasta que comienza la marcha a velocidad 1 desde la linea verde. Entre las dos lineas rojas aproximadamente decide incrementar el ritmo de la marcha a velocidad 2 hasta la última linea verde.

\subsection{Análisis de la marcha}

Para la identificación del instante de la aceleración, procesamos las señales que obtenemos de las IMUs. A continuación expondremos los filtros aplicados y el proceso que seguimos para detectar las continuidades y cambios bruscos.

\subsubsection{Filtrado de ruido}

La etapa de eliminación de ruido es clave a la hora de una detección más certera. Aparte de los filtros integrados en el equipo de captación, se hará uso de un filtrado mediante una wavelet, anteponiéndolo a un filtrado más clásico como el suavizado de spline, debido a que este conserva mejor las características subyacentes de la señal original WAVELET DENOISING. 
Este método de filtrado busca rechazar el ruido mediante el umbral de coeficientes wavelet de la señal ruidosa. Este procesamiento actúa en tres etapas diferentes. En primer lugar, una wavelet analiza la señal y extrae los coeficientes. Para ello utilizaremos la Db20 (Fournier, 2009). Luego los coeficientes extraídos son sometidos a un umbral de rechazo para eliminar el ruido. Finalmente una transformada wavelet inversa reconstruye la señal.

Para implementar dicho modelo utilizamos la función wden de Matlab y los parámetros de configuración son (Fournier, 2009):

1. La señal normalizada de la aceleración de la IMU.

2. Para el umbral hemos utilizado el principio de decisión Stein's Unbiased Risk Estimate (Donoho, 1995).

3. Se ha elegido un umbral suave por la capacidad de reducción del ruido, por oposición el umbral duro es mejor en la conservación.

4. Se ha reescalado estimando el nivel de ruido.

5. El número de nivel de descomposición se ha estimado de forma empírica, escogiendo un nivel de 4.

\subsubsection{Extracción coeficientes $C W T$}

Para el cambio en la velocidad, hacemos un análisis en las variables de aceleración extraídas de las IMUs, y un análisis de tipo frecuencial nos permite detectar los cambios bruscos en la señal. Sin embargo con un análisis en el dominio frecuencial no seríamos capaces de detectar en que instante se producen esas señales características. Estudiar las componentes frecuenciales en el tiempo (CWT) (Capilla, 2006) nos posibilita no perder resolución temporal, y enfatizar sus discontinuidades.

Para el análisis CWT escogemos como wavelet madre la Haar, que nos enfatiza las discontinuidades del conjunto de datos a procesar. Otro aspecto importante para el análisis de dichos componentes es la selección de los coeficientes wavelet. Mediante un banco de filtros de paso banda se hará una división del rango de frecuencias (Tabla 1) y determinamos el número de secciones a coger (Valens, 1999) mediante

$$
J=\log _{2}(N)(1)
$$

donde $\mathrm{J}$ es el número de escalas válidas y $\mathrm{N}$ es la frecuencia de muestreo de la señal.

Para una frecuencia de $30 \mathrm{~Hz}$ se han escogido cinco escalas de la cinco a la ocho, correspondientes a un rango de frecuencias entre $0,12 \mathrm{~Hz}$ y $3.75 \mathrm{~Hz}$. 
Tabla 1. Análisis para la selección de frecuencias en la CWT

\begin{tabular}{|r|cc|c|}
\hline & \multicolumn{2}{|c|}{ Frecuencias } & \multirow{2}{*}{ Muestras } \\
\cline { 2 - 3 } & \multicolumn{1}{|c|}{ Desde (Hz) } & Hasta $(\mathrm{Hz})$ & $\mathrm{N} / 2$ \\
2 & 15,00 & 30,00 & $\mathrm{~N} / 4$ \\
3 & 7,50 & 15,00 & $\mathrm{~N} / 8$ \\
4 & 3,75 & 7,50 & $\mathrm{~N} / 16$ \\
5 & 1,87 & 3,75 & $\mathrm{~N} / 32$ \\
6 & 0,93 & 1,87 & $\mathrm{~N} / 64$ \\
7 & 0,46 & 0,93 & $\mathrm{~N} / 128$ \\
8 & 0,23 & 0,46 & $\mathrm{~N} / 256$ \\
9 & 0,11 & 0,23 & $\mathrm{~N} / 512$ \\
10 & 0,05 & 0,11 & $\mathrm{~N} / 1024$ \\
11 & 0,01 & 0,05 & $\mathrm{~N} / 2048$ \\
\hline
\end{tabular}

El protocolo seguido empieza por el filtrado del ruido según lo explicado en el apartado anterior. Una vez filtradas cada una de las tres aceleraciones se calcula el módulo de dicho vector, obteniendo una señal de aceleración por cada IMU. De la señal resultante se escogen las frecuencias de interés seleccionadas por el filtro de banco. Se calculan los coeficientes en cada rango obteniendo las componentes correspondientes a ese rango de frecuencias. Por último se suman las bandas para calcular la energía contenida en dicho rango, obteniendo el vector que representa las singularidades de la señal a esas escalas. De forma empírica se selecciona el mínimo de la señal como punto de transición obteniendo el punto de cambio de cada IMUs. Por último se promedian los valores (Fig3).
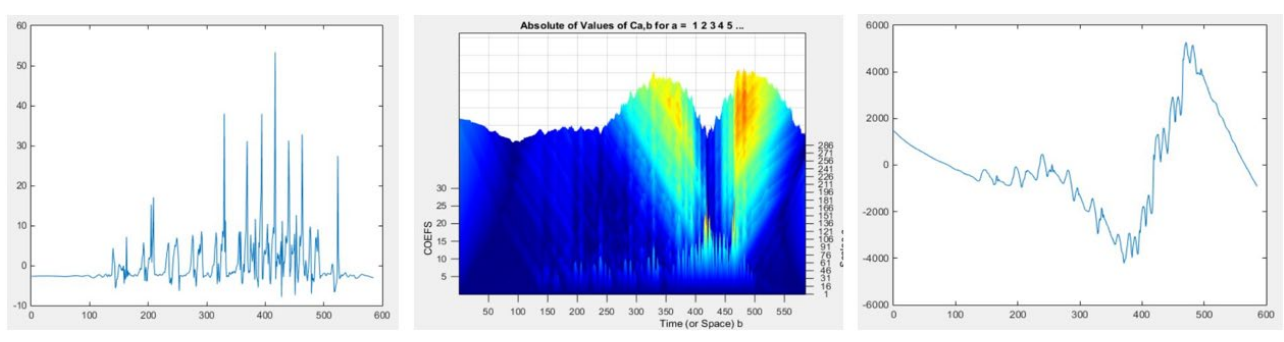

Fig3. A la izquierda señal promediada de las aceleraciones, en el centro los coeficientes extraídos y a la derecha el contenido de la señal a las frecuencias elegidas. 


\subsection{Procesamiento EEG}

Buscamos diferenciar la señal en dos estados, velocidad normal y cambio de velocidad. Para ello planteamos extraer las características más significativas que nos aporten menor correlación posible entre estados. Comentamos los filtros aplicados, así como la justificación de los potenciales seleccionados.

\subsubsection{Preprocesamiento}

Se ha aplicado un filtro Butterworth de orden 8th en el rango de frecuencias 1-40 Hz, al desear evitar el límite de las bandas Alfa (8-14 Hz) y Beta (14-30 Hz). También aplicamos un filtro espacial Laplaciano (McFarland et al., 1997) para reducir la contribución del resto de electrodos a cada canal; usando la información de cada electrodo y la distancia al sensor de interés.

\subsubsection{Extracción de características}

Este estudio trata de clasificar los patrones que codifican la intención de movimiento (Nam et al., 2011). Estos potenciales son endógenos: señales que se generan en el interior del cerebro sin necesidad de estimulación externa. Dependen de la habilidad del usuario para controlar su actividad electrofisiológica. Nuestro interés está en el estudio de los ritmos sensoriomotores, los cuales comprenden dos tipos de variaciones en amplitud, de tipo ERD (desincronización relacionada con el evento) y las ERS (sincronización relacionada con el evento). La primera representa una disminución de la actividad rítmica de duración breve y localizada $1.5 \mathrm{~s}$ antes del evento, la segunda un aumento de la amplitud $0.5 \mathrm{~s}$ después de éste. Estas variaciones son altamente específicas de la banda de frecuencia y no bloqueadas en fase al evento (Pfurtscheller, 1999).

Se selecciona la banda de frecuencia más idónea, separando el espacio a explorar en tres: 8$12 \mathrm{~Hz}, 13-24 \mathrm{~Hz}$ y $25-30 \mathrm{~Hz}$. Para la extracción de características se realiza una transformación de la señal al dominio de la frecuencia mediante el análisis de la potencia de la densidad espectral (PSD), utilizando ventanas Hanning, promediando el valor en todo el rango de frecuencias escogido. Los datos seleccionados se han segmentado en épocas de $1 \mathrm{~s}$ cada $0.2 \mathrm{~s}$ ( $0.8 \mathrm{~s}$ de solapamiento de la señal). En este estudio probamos los resultados de cada una de estas bandas en el modelo de clasificación seleccionado para cada uno de los usuarios (Gómez et al., 2012). 


\subsubsection{Análisis de componentes principales}

Una señal EEG está compuesta por una mezcla de señales de muchas fuentes. Mediante el Análisis de Componentes Independientes (ICA) pretendemos separar la señal en sus componentes principales descartando aquellos que pertenezcan a fuentes de ruido. Para la aplicación del método ICA utilizaremos EEGLAB, importando sus funciones a Matlab SCCN. El proceso seguido para separar las señales es el siguiente:

- Se genera una estructura de datos a partir de las sesiones para que el programa itere sobre estos ficheros.

- Separación de componentes principales utilizando la función rúnica de EEGLAB. Esta función extrae los pesos de los componentes.

- Con los pesos se calcula el espectro en frecuencia de aquellos componentes que vayamos a analizar para una posible substracción.

Por cada componente analizamos su espectro normalizándolo, según las condiciones expuestas más abajo. Solo se extraerán el $10 \%$ de los componentes para preservar la señal.

Los componentes que buscamos eliminar son los pertenecientes a movimientos musculares y oculares, priorizando su eliminación según el orden anterior. El método seguido para discriminar los artefactos (Mayeli et al., 2016) es el que se indica a continuación:

- Las componentes debidas a movimientos se priorizan con un peso del $50 \%$ y en el rango de $0.5-4.5 \mathrm{~Hz}$ se eliminan aquellas que superen un valor de 0.26 .

- Las componentes musculares con un peso del $25 \%$, en el rango de $30-60 \mathrm{~Hz}$ eliminando aquellas que superen el valor de 0.68 .

- Los componentes oculares con un peso del $25 \%$, en el rango de $0.5-3 \mathrm{~Hz}$ de aquellos que superen el valor de 0.22 .

\subsection{Clasificación}

Los datos se procesan de forma offline según dos estados. Los pertenecientes a la clase $\mathrm{C} 1$ serán los datos que interpretamos como marcha normal y los pertenecientes a la clase C2 para la intención de cambio de velocidad.

Para la caracterización de las señales EEG, para nuestros dos estados, se ha utilizado un clasificador del tipo Máquinas de Vector Soporte (SVM). La parametrización del algoritmo, es la utilizada en la mayoría de sistemas BMI: núcleo de base radial, con la configuración de los parámetros $\mathrm{C}=512$ y $\gamma=0.002$ (Flórez et al., 2011). 
Una vez marcado el momento en el que la persona cambia, se etiquetan los datos según pertenezcan a la clase $\mathrm{C} 1$ o $\mathrm{C} 2$. El modelo predictivo se etiqueta, desde el cambio hasta $1.8 \mathrm{~s}$ antes como $\mathrm{C} 2$ y desde $1.8 \mathrm{~s}$ hasta $3.6 \mathrm{~s}$ antes del cambio como $\mathrm{C} 1$.

Los resultados se extraen a partir de una validación cruzada, que valida nuestros resultados de forma estadística, garantizando la independencia de la partición entre datos de entrenamiento y prueba. Se divide en dos subconjuntos, uno de entrenamiento y otro de prueba con tamaño $\mathrm{k}=2$, rotando el conjunto de prueba el proceso de validación cruzada es repetido hasta terminar todas las agrupaciones, sin repetir datos de conjuntos ya seleccionados. La medida utilizada para calcular la calidad de los resultados ha sido el Fscore, mediante

$$
F-\operatorname{score}=2 \frac{\mathrm{P} \times \mathrm{TP}}{\mathrm{TP}+\mathrm{P}}(2)
$$

donde TP son los verdaderos positivos, el número de veces que se acierta la clase frente al total de instancias y $\mathrm{P}$ es la precisión, el número de veces que se clasifica incorrectamente la clase.

\section{Resultados y discusión}

En esta sección exponemos las configuraciones que hemos planteado para el análisis de resultados, así como la justificación de dichas elecciones y por último analizamos la validez de dichos resultados.

\subsection{Resultados en la detección del punto de cambio en la marcha}

Para validar la detección del punto de cambio en el primer experimento con los cuatro sujetos, se compara el momento en el que se le dice al sujeto que cambie de velocidad y el momento en el que detectamos que cambia.

La diferencia en segundos desde que se le dice al usuario que cambie de velocidad, hasta que cambia es inferior al segundo (Tabla 2). Podríamos deducir de los resultados que la determinación del punto no es todo lo precisa que cabría esperar. Sin embargo varias condiciones podrían matizar el resultado. Desde el momento que se indica la aceleración hasta que el sujeto realiza el cambio, existe un retardo; aparte de lo que tarda en acelerar y del tipo de zancada de cada individuo. 
Tabla 2. Diferencias entre el momento marcado y el detectado

\begin{tabular}{|c|c|c|c|c|c|}
\hline Sujetos & \multicolumn{4}{|c|}{ Sesiones } & $\overline{\mathbf{x}} \pm \boldsymbol{\sigma}$ \\
\hline 1 & $0.81 \mathrm{~s}$ & $0.95 \mathrm{~s}$ & $0.92 \mathrm{~s}$ & $0.83 \mathrm{~s}$ & $0.88 \mathrm{~s} \pm 0.07 \mathrm{~s}$ \\
\hline 2 & $0.70 \mathrm{~s}$ & $0.98 \mathrm{~s}$ & $0.56 \mathrm{~s}$ & $1.26 \mathrm{~s}$ & $0.88 \mathrm{~s} \pm 0.31 \mathrm{~s}$ \\
\hline 3 & $0.81 \mathrm{~s}$ & $0.50 \mathrm{~s}$ & $0.56 \mathrm{~s}$ & $0.46 \mathrm{~s}$ & $0.58 \mathrm{~s} \pm 0.16 \mathrm{~s}$ \\
\hline 4 & $0.35 \mathrm{~s}$ & $-0.07 \mathrm{~s}$ & $0.49 \mathrm{~s}$ & $0.83 \mathrm{~s}$ & $0.40 \mathrm{~s} \pm 0.37 \mathrm{~s}$ \\
\hline
\end{tabular}

\subsection{Resultados en la clasificación de señales EEG}

Seleccionamos las bandas más adecuadas con 11 electrodos FC1, FCz, FC2, C3, C1, Cz, C2, $\mathrm{C} 4, \mathrm{CP} 1, \mathrm{CPz}, \mathrm{CP} 2$. Se estudian las bandas correspondientes a $8-12 \mathrm{~Hz}, 13-24 \mathrm{~Hz}$ y $25-30 \mathrm{~Hz}$, extrayendo una característica por electrodo.

Los espectros de las distintas bandas nos pueden estar indicando donde se produce la máxima distinción de los potenciales ERD/ERS (Ang y Guan, 2013) para cada sujeto (Tabla 3). El Sujeto 5 encuentra mayor acierto en las bajas, mientras que los otros dos maximizan el acierto en las altas. Debido a este análisis se ha decidido utilizar para los Sujetos 6 y 7 las frecuencias correspondientes a la zona Beta, mientras que para el Sujeto 1, las correspondientes a la zona Alfa.

Tabla 3. Resultados para las diferentes bandas

\begin{tabular}{|c|c|c|c|c|c|c|}
\hline \multirow{2}{*}{ Sujetos } & \multicolumn{2}{|c|}{ Banda: 8-13Hz } & \multicolumn{2}{c|}{ Banda: 13-24Hz } & \multicolumn{2}{c|}{ Banda: 25-30Hz } \\
\cline { 2 - 7 } & F-score C1(\%) & F-score C2 (\%) & F-score C1 (\%) & F-score C2 (\%) & F-score C1 (\%) & F-score C2 (\%) \\
\hline 5 & 56.35 & 51.75 & 51.03 & 49.79 & 49.79 & 49.37 \\
6 & 45.16 & 50.21 & 43.75 & 45.69 & 53.57 & 55.17 \\
7 & 54.84 & 51.72 & 50.22 & 53.82 & 54.24 & 55.74 \\
\hline$\overline{\mathbf{x}} \pm \boldsymbol{\sigma}$ & $54.84 \pm 6.07$ & $51.72 \pm 0.88$ & $50.22 \pm 3.98$ & $49.79 \pm 4.06$ & $53.57 \pm 2.39$ & $55.17 \pm 3.52$ \\
\hline
\end{tabular}

Se han probado (Rodríguez, 2017) distintas configuraciones correspondientes a la zona central y homúnculo motor para la configuración de electrodos 1 , zona central y la zona motora para la configuración 2 y la configuración 3 zona central más lóbulo parietal.

Tabla 4. Resultados para las configuraciones indicadas sin eliminación de artefactos

\begin{tabular}{|c|c|c|c|c|c|c|}
\hline \multirow{3}{*}{ Sujetos } & \multicolumn{5}{|c|}{ Sin ICA } \\
\cline { 2 - 7 } & \multicolumn{2}{|c|}{ Configuración 1 } & \multicolumn{2}{c|}{ Configuración 2 } & \multicolumn{2}{c|}{ Configuración 3 } \\
\cline { 2 - 7 } & F-score C1 (\%) & F-score C2 (\%) & F-score C1 (\%) & F-score C2 (\%) & F-score C1 (\%) & F-score C2 (\%) \\
\hline 5 & 56.35 & 51.75 & 57.26 & 59.35 & 52.72 & 53.11 \\
6 & 53.57 & 55.17 & 57.64 & 57.27 & 57.63 & 54.55 \\
7 & 54.24 & 55.74 & 46.67 & 46.67 & 60.99 & 66.15 \\
\hline$\overline{\mathbf{x}} \pm \boldsymbol{\sigma}$ & $54.24 \pm 1.45$ & $55.17 \pm 2.16$ & $57.26 \pm 6.23$ & $57.27 \pm 6.80$ & $57.63 \pm 4.16$ & $54.55 \pm 7.15$ \\
\hline
\end{tabular}


Probamos dichas configuraciones sin (Tabla 4) y con filtro ICA. Los mejores resultados para los sujetos 5 y 6 se consiguen para la configuración 2, para el 7 se consiguen en la configuración 3. Como podemos observar (Tabla 5) los valores no mejoran, sino que incluso empeoran. Es posible que el algoritmo este quitando información esencial, no obstante eliminar las componentes debidas al movimiento es imprescindible puesto que sino podríamos estar clasificando artefactos, en vez de los potenciales que se buscan.

Tabla 5. Resultados para las configuraciones indicadas con eliminación de artefactos

\begin{tabular}{|c|c|c|c|c|c|c|}
\hline \multirow{3}{*}{ Sujetos } & \multicolumn{3}{|c|}{ Con ICA } \\
\cline { 2 - 7 } & \multicolumn{2}{|c|}{ Configuración 1 } & \multicolumn{2}{c|}{ Configuración 2 } & \multicolumn{2}{c|}{ Configuración 3 } \\
\cline { 2 - 7 } & F-score C1 (\%) & F-score C2 (\%) & F-score C1 (\%) & F-score C2 (\%) & F-score C1 (\%) & F-score C2 (\%) \\
\hline 5 & 54.24 & 55.74 & 56.17 & 57.96 & 51.85 & 50.63 \\
6 & 53.81 & 55.79 & 52.73 & 55.93 & 54.22 & 55.41 \\
7 & 57.03 & 50.89 & 54.98 & 50.66 & 54.69 & 52.7 \\
\hline$\overline{\mathbf{x}} \pm \boldsymbol{\sigma}$ & $54.24 \pm 1.75$ & $55.74 \pm 2.81$ & $54.98 \pm 1.75$ & $55.93 \pm 3.77$ & $54.22 \pm 1.52$ & $52.7 \pm 2.40$ \\
\hline
\end{tabular}

\section{Conclusión}

En este trabajo se ha creado una interfaz BMI para la detección de la intención del cambio de velocidad a partir de la adquisición de señales EEG y el estudio de la marcha. Abordamos los protocolos a seguir y las especificaciones para la creación de un modelo de predicción combinando el análisis de la marcha, con el procesamiento de los potenciales sensoriomotores. Para el análisis de la marcha se ha utilizado el análisis CWT, con filtrado de ruido y selección de la banda de frecuencias más óptima (Fournier, 2009). Los resultados obtenidos (Tabla 3) nos aportan un error menor a $1 \mathrm{~s}$, que achacamos al tiempo de reacción y al tiempo de aceleración. La señal EEG, es filtrada y suavizada extrayendo el poder espectral para ciertas bandas de frecuencia. Una vez separada según la clase se entrena un modelo de clasificación SVM para cada una de las configuraciones. El mayor acierto para ambas clases conseguido en la configuración 2 para sujetos 5, 6 y la configuración 3 para el sujeto 7 según la (Tabla 4) es del $58.31 \% \pm 0.61$. El resultado no es lo suficientemente aceptable, debido a que la arquitectura BMI no permitiría el control óptimo para que un modelo de rehabilitación sea consistente. El algoritmo de filtrado no ha aportado resultados satisfactorios. El eliminar ciertas componentes (Mayeli et al., 2016) ha empeorado los resultados (Tabla 5) para cada configuración (Tabla 3) un 1.6\% de media, empeorando también los mejores resultados globales expuestos anteriormente. Al no ser resultados positivos (Tabla 5), no podemos descartar que los artefactos de movimiento infieran acierto en la clasificación cuando no aplicamos el filtro ICA (Tabla 4). 
En resumen, nuestros resultados no son concluyentes pero asientan las bases para el futuro de una interfaz más eficaz. Existiendo un gran abanico de posibilidades para la mejora: aspectos como la selección de las frecuencias optimas por cada canal, la selección de las ventanas que mayor acierto proporcionan para cada usuario... Con una tasa de acierto mayor el paso siguiente sería un modelo online de predicción.

\section{Agradecimientos}

Esta investigación ha sido realizada en el marco del proyecto Walk - Control de exoesqueletos de miembro inferior mediante interfaces cerebro-máquina para asistir a personas con problemas de marcha (RTI2018-096677-B-I00), financiado por el Ministerio de Ciencia, Innovación y Universidades (MCIU), la Agencia Estatal de Investigación (AEI) y la Unión Europea a través del Fondo Europeo de Desarrollo Regional (FEDER).

\section{Referencias}

ANG, K. K., and Guan, C. (2013). "Brain-computer interface in stroke rehabilitation". J. Comput. Sci. Eng. 7, 139-146.

BRAIN PRODUCTS. Products \& Application $<$ https://www.brainproducts.com/products_by_type.php?tid=1 $>$ [ Consulta : 11 de agosto de 2017]

CAPILLA, C. (2006). "Application of the Haar wavelet transform to detect microseismic signal arrivals". Journal of applied geophysics, 59(1), 36-46.

DOBKIN, B. H. (2007). "Brain-computer interface technology as a tool to augment plasticity and outcomes for neurological rehabilitation”. J. Physiol. 579, 637-642.

DONOHO, D. L., \& Johnstone, I. M. (1995). "Adapting to unknown smoothness via wavelet shrinkage." Journal of the american statistical association, 90(432), 1200-1224.

EEGLAB. Descomposing Data Using ICA. $<$ https://sccn.ucsd.edu/wiki/Chapter_09:_Decomposing_Data_Using_ICA $>$ [ Consulta : 13 de agosto de 2017]

FLOREZ, F., Azorín, J. M., Iáñez, E., Úbeda, A., \& Fernández, E. (2011, October). Development of a Low-cost SVM-based Spontaneous Brain-computer Interface. In IJCCI (NCTA) (pp. 415-421).

FOURNIER, H. (2009). “Automated Processing of GPS/MEMS-IMU Data for Position”, Velocity and Attitude Determination (No. STUDENT).

GOMEZ, J., Aguilar, M., Horna, E., \& Minguez, J. (2012). "Quantification of event-related desynchronization/synchronization at low frequencies in a semantic memory task". Annual International Conference of the IEEE Engineering in Medicine and Biology Society (pp. 2522-2526). IEEE. 
GORGEY, A. S. (2018). Robotic exoskeletons: The current pros and cons. World journal of orthopedics, 9(9), 112.

HORTAL, E., Úbeda, A., Iáñez, E., Azorín, J. M., \& Fernández, E. (2016). "EEG-based detection of starting and stopping during gait cycle". International journal of neural systems, 26(07), 1650029.

JIANG, N., Gizzi, L., Mrachacz-Kersting, N., Dremstrup, K., \& Farina, D. (2015). “A brain-computer interface for single-trial detection of gait initiation from movement related cortical potentials". Clinical Neurophysiology, 126(1), 154-159.

MATHWORKS. Wavelet Denoising. <https://es.mathworks.com/help/wavelet/ug/waveletdenoising.html $>$ [Consulta : 14 de agosto de 2017]

MAYELI, A., Zotev, V., Refai, H., \& Bodurka, J. (2016). "Real-time EEG artifact correction during fMRI using ICA". Journal of neuroscience methods, 274, 27-37.

MCFARLAND, D. J., McCane, L. M., David, S. V., \& Wolpaw, J. R. (1997). "Spatial filter selection for EEG-based communication”. Electroencephalography and clinical Neurophysiology, 103(3), 386394.

MURIE-FERNANDEZ, M., IRIMIA, P., MARTINEZ-VILA, E., MEYER, M. J., \& TEASELL, R. (2010). "Neuro-rehabilitation after stroke" Neurología, vol. 25, 3, 189-196.

NAM, C. S., Jeon, Y., Kim, Y.-J., Lee, I., and Park, K. (2011)."Movement imagery-related lateralization of event-related (de) synchronization (ERD/ERS): motor-imagery duration effects". Clin. Neurophysiol. 122, 567-577.

PFURTSCHELLER, G., \& Da Silva, F. L. (1999). Event-related EEG/MEG synchronization and desynchronization: basic principles. Clinical neurophysiology, 110(11), 1842-1857.

RODRIGUEZ-Ugarte, M., Iáñez, E., Ortíz, M., \& Azorín, J. M. (2017). "Personalized offline and pseudo-online BCI models to detect pedaling intent". Frontiers in neuroinformatics, 11, 45.

TECHNAID, Manual de usuario Sistema de Captura de Movimiento Tech-MCS V.3.0. Revisión : 25 de Junio de 2014

TOFFANIN, Paolo, et al. "Rethinking neural efficiency: Effects of controlling for strategy use." Behavioral neuroscience 121.5 (2007): 854.

VALENS, C. (1999). “A really friendly guide to wavelets”. ed. Clemens Valens. 


\title{
Posible efecto de interacción entre el paradigma de presentación y el conjunto de estímulos en un teclado virtual controlado a través de una interfaz cerebro-ordenador basada en P300
}

\author{
Fernández-Rodríguez, Álvaro; Medina-Juliá, María Teresa; Velasco-Álvarez, \\ Francisco y Ron-Angevin, Ricardo \\ Departamento de Tecnología electrónica, Universidad de Málaga, España, afernandezrguez@uma.es, \\ maytemed@uma.es, fvelasco@dte.uma.es,rron@uma.es.
}

\begin{abstract}
Many works in visual P300-based spellers have presented novel stimuli that improve the performance under row-column paradigm (RCP). On the other hand, the rapid serial visual presentation (RSVP) has been presented as an adequate option for patients. Thus, the aim of this work is to assess two set of stimuli under two presentation paradigms: RCP and RSVP. Sixteen participants controlled four conditions: letters in RCP, pictures in RCP, letters in RSVP and pictures in RSVP. The results showed that the improvement effect given by pictures was higher under RCP than under RSVP. Therefore, this work probes that the improvements related to alternative stimuli under $R C P$ may not be directly transferred to the RSVP.
\end{abstract}

Keywords: brain-computer interface (BCI), speller, P300, row-column paradigm (RCP), rapid serial visual presentation (RSVP).

\section{Resumen}

Muchos trabajos en spellers basados en señal P300 visual han presentado estímulos novedosos que mejoraron el rendimiento utilizando el paradigma fila-columna (RCP). Sin embargo, la presentación visual serial rápida (RSVP) se ha mostrado como una opción adecuada para pacientes. Por tanto, el objetivo de este trabajo es evaluar dos conjuntos de estímulos en dos modos de presentación: RCP y RSVP. Dieciséis participantes controlaron cuatro spellers: letras en RCP, imágenes en RCP, letras en RSVP e imágenes en RSVP. Los resultados mostraron que el efecto de mejora dado por las imágenes fue mayor en RCP que en RSVP. Por lo tanto, este trabajo prueba que las mejoras relacionadas con estímulos alternativos bajo RCP pueden no ser transferidas directamente al RSVP.

Palabras clave: interfaz cerebro-ordenador, teclado, P300, presentación. 
Posible efecto de interacción entre el paradigma de presentación y el conjunto de estímulos en un teclado virtual controlado a través de una interfaz cerebro-ordenador basada en P300

\section{Introducción}

El objetivo principal de la tecnología asistencial es mejorar la calidad de vida de sus potenciales usuarios. Estos usuarios son pacientes afectados por determinadas lesiones o enfermedades que causan un deterioro de las capacidades motoras. Un ejemplo de estas enfermedades es la esclerosis de lateral amiotrófica (ELA), causante de un progresivo y severo deterioro de la motricidad (Patterson y Grabois, 1986). En algunos casos, estos pacientes alcanzan el llamado estado de enclaustramiento completo (CLIS, de complete locked-in state), en el que incluso la capacidad de controlar la mirada ha sido perdida (Bauer, Gerstenbrand, y Rumpl, 1979; Murguialday et al., 2011). Sin embargo, incluso en estos casos extremos, la capacidad cognitiva de dichos pacientes no tiene por qué estar necesariamente afectada (Fuchino et al., 2008).

Según ha informado (Lemoignan y Ells, 2010), para la mayoría de los pacientes de ELA, la habilidad para comunicarse fue el factor más importante para decidir si deseaban utilizar (o continuar utilizando) la ventilación asistida, por lo que convierte a la comunicación en un factor clave de la calidad de vida de los pacientes. Las interfaces cerebro-ordenador (BCI, de brain-computer interface) son un tipo de tecnología que utiliza la actividad cerebral del usuario para establecer un canal de comunicación entre su cerebro y el dispositivo que desea control (Birbaumer, 2006; Wolpaw, Birbaumer, McFarland, Pfurtscheller, y Vaughan, 2002). Estos dispositivos pueden ser, por ejemplo, una silla de ruedas, un sistema domótico o un teclado virtual (Corralejo, Hornero, y Álvarez, 2011; Farwell y Donchin, 1988; Zhang et al., 2017). Ya que un sistema BCI no requiere de ningún tipo de motricidad para ser controlado, puede ser una opción adecuada para aquellos pacientes que no puedan realizar ningún movimiento, pero mantengan sus capacidades cognitivas. Debido a su relativamente bajo coste y adecuada resolución temporal, la tecnología más ampliamente utilizada para la grabación de la señal del cerebro es la electroencefalografía (EEG).

Una de las principales aplicaciones desarrolladas con esta tecnología son los teclados virtuales, también conocidos como spellers. Estos spellers permiten a un usuario seleccionar entre un conjuntos de símbolos con fines de comunicación (véase Rezeika et al. (2018) para una revisión extendida sobre spellers manejados a través de $\mathrm{BCI}$ ). La señal del cerebro más utilizadas para esta aplicación es el potencial P300. El P300 es una deflexión positiva en el voltaje de la señal EEG alrededor de 300 ms después de la presentación de un estímulo objetivo (Sutton, Braren, Zubin, y John, 1965). Por tanto, el usuario sólo tiene que mantener su atención en el estímulo objetivo, evocando un P300 cada vez que aparezca dicho estímulo. Posteriormente, el sistema reconoce el potencial P300 y selecciona el símbolo que se relaciona con dicho potencial. Por último, el sistema ejecuta el comando asociado a ese estímulo (p. ej., escribir una letra, borrar el último carácter o autocompletar una palabra). 
El paradigma de presentación más utilizado por los investigadores ha sido el paradigma de fila-columna (RCP, de row-column paradigm), propuesto por Farwell y Donchin (1988). En este paradigma, los estímulos se muestran en una matriz de símbolos, lo cuáles son iluminados de forma conjunta por filas y columnas. Sin embargo, debido a la distancia entre los estímulos, el modo de presentación RCP requiere que los usuarios deben tener algún control ocular, ya que el rendimiento se ve severamente afectado en caso contrario (Brunner et al., 2010; Treder y Blankertz, 2010). Además del modo de presentación RCP, hay otras alternativas de presentación de estímulos que no requieren movilidad ocular. El paradigma más utilizado que no requiere movilidad ocular es el de presentación visual serial rápida (RSVP, de rapid serial visual presentation) (Sato y Washizawa, 2016; Treder y Blankertz, 2010).

Debido a la dificultad de control de la mirada en algunos pacientes, el paradigma RSVP paradigma podría ser más conveniente que el RCP para dicha población. Sin embargo, la mayoría de investigaciones han quedado centradas en spellers dependientes del control ocular (según Rezeika et al. (2018), un 84,4\% de teclados virtuales basados en P300 BCI han requerido movilidad ocular). La investigación sobre spellers basados en P300 bajo RCP ha sido extensa y productiva; se han realizado diversos estudios sobre el efecto de diferentes estímulos visuales, los tiempos de presentación o pequeñas variaciones en la modalidad de presentación (Li, Liu, Li, y Bai, 2015; Sellers, Krusienski, McFarland, Vaughan, y Wolpaw, 2006; Townsend et al., 2010). Sin embargo, debe ser cuestionado si estos hallazgos realizados en RCP pueden ser transferidos a paradigmas que no requieran control oculomotor, como el RSVP.

Hasta la fecha, se han presentado diversos estímulos que parecen mejorar el rendimiento de forma significativa bajo RCP (Fernández-Rodríguez, Velasco-Álvarez, y Ron-Angevin, 2018; Jones y Sellers, 2019; Kellicut-Jones y Sellers, 2018). Sin embargo, el efecto de mejora producido por estos estímulos bajo RCP no ha sido comparado frente a un posible efecto de mejora en RSVP. Por tanto, el objetivo del presente estudio es poner a prueba dos conjuntos de estímulos (letras e imágenes) bajo dos paradigmas de presentación diferentes (RSVP y $\mathrm{RCP}$ ) para evaluar las diferencias entre las condiciones.

\section{Método}

\subsection{Participantes}

El experimento fue llevado a cabo por 16 participantes $(22.33 \pm 3.98$ años, 5 hombres $)$ quienes declararon tener una visión normal o corregida. Cinco participantes tenían experiencia previa en control de sistemas BCI. El estudio fue aprobado por el Comité Ético 
Posible efecto de interacción entre el paradigma de presentación y el conjunto de estímulos en un teclado virtual controlado a través de una interfaz cerebro-ordenador basada en P300

de la Universidad de Málaga. Según los informes, ninguno de los participantes declaró antecedentes de enfermedad neurológica o psiquiátrica, o que tomaran algún medicamento regularmente.

\subsection{Adquisición de datos y procesamiento de la señal}

El EEG fue registrado con una frecuencia de muestreo de $250 \mathrm{~Hz}$ usando las posiciones de los electrodos: Fz, Cz, Pz, Oz, P3, P4, PO7 y PO8, según el sistema internacional 10-20. Todos los canales fueron referenciados a TP8, mientras que la tierra fue localizada en AFz. Las señales fueron amplificadas por un amplificador acti-CHamp (Brain Products GmbH, Múnich, Alemania) y un filtrado de paso-banda de 0.1-9 Hz. Todos los aspectos de recopilación de datos EEG y procesamiento fueron controlados por el sistema de BCI2000 (Schalk, McFarland, Hinterberger, Birbaumer, y Wolpaw, 2004). Dicho software empleó el análisis lineal discriminante de pasos hacia delante (SWLDA, de step-wise lineal discriminant analysis) de los datos obtenido a través del EEG para crear el clasificador que permitiría realizar la selección de comandos online.

\subsection{Condiciones}

El software utilizado para diseñar la interfaz fue el UMA-BCI speller, que sirve como un front-end de BCI2000 de fácil manejo (Velasco-Álvarez et al., 2019). El presente trabajo emplea cuatro condiciones, basadas en la combinación de los dos paradigmas (RCP y RSVP) y dos configuraciones de estímulos (letras e imágenes con letras). De manera específica, las cuatro condiciones presentadas fueron: i) letras en RCP (L-RCP, de letters in row-column paradigm), ii) imágenes en RCP (P-RCP, de pictures in row-column paradigm), iii) letras RSVP (L-RSVP, de letters in rapid serial visual presentation), e iv) imágenes en RSVP (PRSVP, de pictures in rapid serial visual presentation) (Figura 1). Las condiciones fueron exhibidas en una pantalla de 15.6 - pulgadas $(39,6 \mathrm{~cm})$ a una distancia de $60 \mathrm{~cm}$ y a una tasa de refresco de $60 \mathrm{~Hz}$. Para todas las condiciones, cada estímulo fue iluminado durante 192 ms, siendo el tiempo entre iluminación de estímulos de $96 \mathrm{~ms}$; por tanto, el tiempo entre aparición de estímulos fue de 288 ms. Fue establecida una pausa de 3968 ms entre cada selección.

Por un lado, las condiciones bajo RCP - con un tamaño de matriz de $3 \times 3$ - se basaron en el paradigma anteriormente mencionado de Farwell y Donchin (1988). Por otra parte, para el RSVP, se presentaron sólo los estímulos intermitentes (es decir, aquellos que se iluminaban durante $96 \mathrm{~ms}$ ), localizados en el centro de la pantalla. Todos los estímulos (las letras y las imágenes editadas con letras) fueron creadas utilizando el software para la edición de imágenes GIMP (www.gimp.org). Para todas las condiciones, la fuente usada para las letras 
fue Arial (pt 700) en mayúsculas y tenían un tamaño de alrededor de $3 \mathrm{~cm} \mathrm{x} 3.5 \mathrm{~cm}$, dependiendo de la letra. Con respecto a las imágenes, que sólo se utilizan en P-RSVP y PRCP, su tamaño era igual a 4,7 cm x 3,5 cm (187 px $\times 140$ px). La distancia entre centros de estímulos adyacentes, fueron $6,7 \mathrm{~cm}$ en sentido horizontal y $5,5 \mathrm{~cm}$ en sentido vertical. Las

L-RSVP

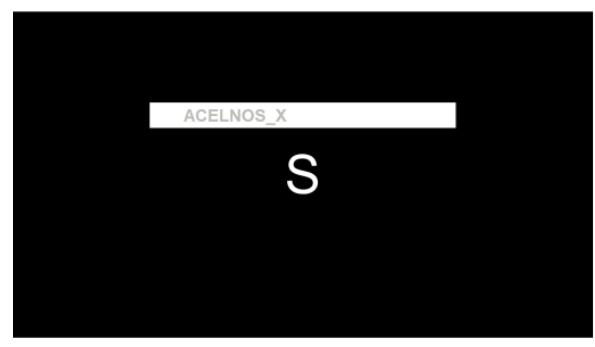

L-RCP

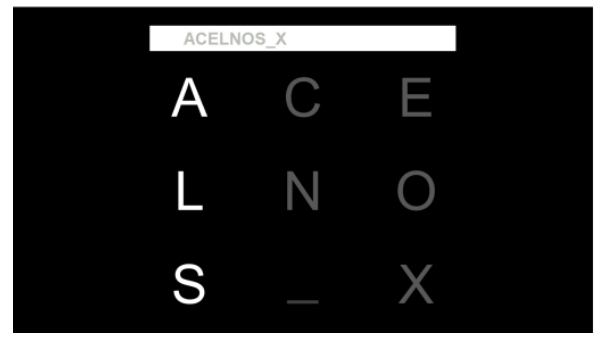

P-RSVP

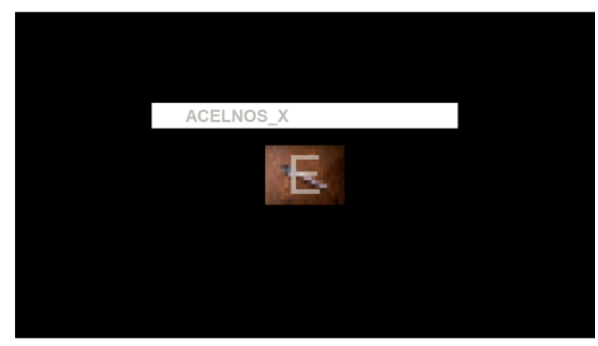

P-RCP

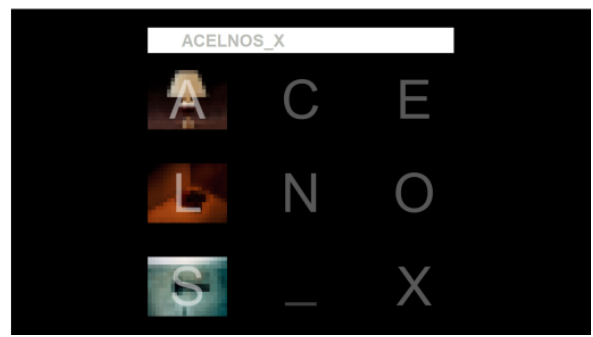

Fig. 1 Condiciones probadas en el estudio: letras en RSVP (L-RSVP), imágenes en RSVP (P-RSVP), letras en $R C P(L-R C P)$ e imágenes en $R C P(P-R C P)$. Debido a los derechos de copyright, las imágenes han sido pixeladas.

letras en las condiciones con imágenes se encontraban en el centro de las imágenes con un nivel de opacidad del 50\%. El propósito de agregar la letra a las condiciones con imágenes era la de facilitar con qué letra se encontraba asociada cada imagen, algo especialmente útil para la condición P-RSVP. Sin embargo, como se mencionó anteriormente, el participante tuvo una pausa de $3968 \mathrm{~ms}$ en el cual él podría visualizar un documento impreso que se coloca al lado de la pantalla, el cual indicaba a qué imagen corresponde cada letra.

Con respecto a las imágenes, fueron obtenidas del International Affective Picture System (IAPS) (Lang, P. J., Bradley, M. M., y Cuthbert, 2008). Fueron escogidas aquellas imágenes con la puntuación más baja en activación y cuyo tamaño fuera similar al anteriormente mencionado $(187 \mathrm{px} \times 140 \mathrm{px})$. Las imágenes seleccionadas según la codificación del IAPS para la matriz fueron (orden de izquierda-derecha y arriba-abajo): 7175, 7010, 7004, 7031, 7020, 7950, 7110, 7080 y 7006 . 
Posible efecto de interacción entre el paradigma de presentación y el conjunto de estímulos en un teclado virtual controlado a través de una interfaz cerebro-ordenador basada en P300

\subsection{Procedimiento}

El experimento se llevó a cabo en una habitación aislada donde sólo el participante estuvo presente en el momento de realizar la prueba. La sesión consistía en dos ejercicios: una tarea de calibración para adaptar el sistema al usuario, y una tarea de escritura - o tarea en línea en la que el usuario realmente controlaba la interfaz.

Se utilizó un diseño intrasujeto, también llamado de medidas repetidas, por lo que todos los usuarios pasaron a través de todas las condiciones experimentales. Cada condición fue realizada en tres bloques de nueve selecciones para la tarea de calibración, y en un solo bloque de, al menos, 15 selecciones para la tarea de escritura en línea (los usuarios pueden cometer errores y corregirlos, por lo que el número de selecciones podría ser más de 15). El usuario pasaba a la siguiente condición cuando terminaba con los tres bloques de calibración y el bloque de escritura en línea de la condición anterior. El orden de presentación de las condiciones fue elegido a través de un diseño de cuadrado latino para evitar cualquier efecto no deseado, como el aprendizaje o la fatiga.

Para la tarea de calibración, el orden de selección de símbolo fue de izquierda a derecha y de arriba a abajo para cada bloque en la tarea de calibración (es decir, A, C, E, L, N, O, S, _ y $\mathrm{X}$ ). El número de secuencias por selección fue igual a 5, por lo que cada símbolo fue iluminado 10 veces en RCP ( 5 de la fila y 5 de la columna) y 5 veces en RSVP. El participante fue instruido para contar mentalmente dichas iluminaciones. El tiempo necesario para completar una secuencia fue de $2784 \mathrm{~ms}$ en RSVP y de $1632 \mathrm{~ms}$ en RCP. Por otro lado, la frase demandada para la tarea de escritura fue "CENO_EN_LA_CASA". Mientras que para la tarea de calibración el número de secuencias fue el mismo para todos los participantes, el escogido para la tarea de escritura dependió del rendimiento obtenido en dicha calibración. La secuencia elegida para la tarea de escritura en línea fue aquella en la cual el usuario obtuvo su mejor accuracy por primera vez en la tarea de calibración.

Después de que el usuario terminara con la tarea de escritura en línea en cada condición, tuvo que responder con puntuaciones de 0 a 10 (de muy bajo a muy alto) a las siguientes preguntas: i) esfuerzo necesario para realizar la tarea correctamente, y ii) nivel de fatiga producido por el control de la interfaz. Asimismo, también debía responder a: i) paradigma de presentación preferido (siendo las opciones RSVP y RCP), ii) estímulos preferidos (siendo las opciones letras e imágenes), y iii) condición favorita (siendo las opciones L-RSVP, P-RSVP, L-RCP y P-RCP).

\subsection{Evaluación}

Fueron utilizadas dos variables dependientes para evaluar el efecto de los estímulos en cada 
modo de presentación: i) la accuracy del sistema (es decir, el número de selecciones correctas entre el número total de selecciones), y ii) la tasa de transferencia de información (ITR, de information transfer rate, bits/min) basada en la fórmula presentada por Wolpaw, Ramoser, McFarland, y Pfurtscheller (1998). Tal y como se ha utilizado en la literatura, el ITR no considera el tiempo de pausa entre selecciones. Para analizar los efectos del modo de presentación y del estímulo, se hicieron varios análisis de la varianza (ANOVA) para buscar diferencias significativas. Para las comparaciones múltiples, se aplicó el método de corrección de Bonferroni.

\section{Resultados}

\subsection{Tarea de calibración}

Tabla 1. Accuracy (\%, media \pm desviación estándar) media obtenida para cada una de las condiciones y sus respectivas comparaciones múltiples.

\begin{tabular}{ccccc}
\hline \multirow{2}{*}{ Secuencia } & \multicolumn{4}{c}{ Speller } \\
\cline { 2 - 5 } & $(1) \mathrm{L}-$ RSVP & $(2) \mathrm{P}-$ RSVP & $(3) \mathrm{L}-\mathrm{RCP}$ & $(4) \mathrm{P}-\mathrm{RCP}$ \\
\hline 1 & $62.72 \pm 12.65^{4}$ & $62.22 \pm 13^{4}$ & $73.59 \pm 15.77^{4}$ & $90.13 \pm 7.36^{1,2,3}$ \\
2 & $80.25 \pm 16.12^{4}$ & $78.95 \pm 12.85^{3,4}$ & $89.39 \pm 9.79^{2,4}$ & $98.52 \pm 2.34^{1,2,3}$ \\
3 & $86.17 \pm 15.87$ & $88.88 \pm 10.07^{4}$ & $95.56 \pm 5.97$ & $98.77 \pm 2.68^{2}$ \\
4 & $91.61 \pm 14.17$ & $94.57 \pm 7.52$ & $95.8 \pm 5.21$ & $99.51 \pm 1.3$ \\
5 & $92.34 \pm 13.96$ & $96.05 \pm 4.94$ & $96.79 \pm 4.17$ & $99.75 \pm 0.96$
\end{tabular}

Nota: letras en RSVP (L-RSVP), imágenes en RSVP (P-RSVP), letras en RCP (L-RCP) e imágenes en RCP (P-RCP). Las diferencias significativas $(p<0.05)$ han sido marcadas con un superíndice para señalar con qué condición ha sido diferente (1 para L-RSVP, 2 para P-RSVP, 3 para L-RCP, y 4 para P-RCP). Ha sido aplicada la corrección de Bonferroni.

Accuracy. Fue realizado un ANOVA $(2 \times 2 \times 5)$ de medidas repetidas con los siguientes factores: presentación (RSVP y RCP), estímulo (letras e imágenes) y secuencia (5 secuencias). El análisis mostró un efecto principal de la presentación $(F(1,14)=36,644, p$ $<0,001)$ y de la secuencia $(F(4,56)=149,715, p<0,001$. Por tanto, se podría afirmar que el modo de presentación RCP $(93,98 \pm 10,09 \%)$ ofreció una mayor accuracy que el RSVP $(83,72 \pm 16,79 \%)$, y que dicha accuracy aumentó con el trascurso de las secuencias. Además, los efectos de interacción presentación $\times \operatorname{secuencia~}(F(4,56)=15,453, p<0,001)$ y paradigma $\times$ estímulo $\times$ secuencia $(F(4,56)=10,094, p=0,001)$ resultaron significativos El efecto de la interacción paradigma $\times$ secuencia sugiere que las diferencias entre RCP y RSVP se reducen según aumenta el número de secuencias. Por otra parte, el efecto de 
Posible efecto de interacción entre el paradigma de presentación y el conjunto de estímulos en un teclado virtual controlado a través de una interfaz cerebro-ordenador basada en P300

interacción paradigma $\times$ estímulo $\times$ secuencia sugiere que el efecto de la presentación depende de los estímulos utilizados y el número de secuencias. De manera adicional, se ha realizado un análisis comparando la accuracy de las diferentes condiciones individualmente (es decir, L-RSVP, P-RSVP, L-RCP y P-RCP) para cada una de las secuencias. Los resultados de este análisis se muestran en la tabla 1.

ITR. Fue realizado un ANOVA $(2 \times 2 \times 5)$ de medidas repetidas con los siguientes factores: presentación (RSVP y RCP), estímulo (letras e imágenes) y secuencia (5 secuencias). El análisis mostró un efecto principal para cada uno de los factores: presentación $(F(1,14)=$ $579,711, p<0,001)$, estímulo $(F(1,14)=13,678 ; p=0,002)$ y secuencia $(F(4,56)=200,606$, $p<0,001$. Por tanto, se podría afirmar que el modo de presentación de RCP $(42,52 \pm 22.85$ bits/min) y las imágenes $(33,07 \pm 24,21 \mathrm{bits} / \mathrm{min})$ ofrecieron un ITR significativamente mayor que el modo $\operatorname{RSVP}(17,94 \pm 7,85 \mathrm{bits} / \mathrm{min})$ y las letras $(27,39 \pm 16,89 \mathrm{bits} / \mathrm{min})$, respectivamente. Además, el ITR fue menor a medida que aumentaba el número de secuencias. En referencia a los efectos de interacción, todos resultaron significativos: presentación $\times$ estímulo $(F(1,14)=10,003, p=0,007)$, presentación $\times$ secuencia $(F(4,56)$ $=95,208, p<0,001)$, estímulo $\times$ secuencia $(F(4,56)=11,809, p=0,002)$ y presentación $\times$ estímulo $\times$ secuencia $(F(4,56)=18,918, p<0,001$. El efecto de interacción paradigma $\times$ estímulo $\times$ secuencia sugiere que el efecto de la presentación depende de los estímulos utilizados y el número de secuencias. Como en la accuracy, se realizó un ANOVA para cada secuencia considerando cada teclado individualmente. Los resultados de este análisis se muestran en la tabla 2.

Tabla 2. Ratio de transferencia de información (bits/min, media \pm desviación estándar) medio obtenida para cada una de las condiciones y sus respectivas comparaciones múltiples.

\begin{tabular}{ccccc}
\hline \multirow{2}{*}{ Secuencia } & \multicolumn{4}{c}{ Speller } \\
\cline { 2 - 5 } & (1) L-RSVP & (2) P-RSVP & (3) L-RCP & (4) P-RCP \\
\hline 1 & $24.68 \pm 10.05^{3,4}$ & $24.33 \pm 9.89^{3,4}$ & $59.84 \pm 23.15^{1,2,4}$ & $89.76 \pm 14.59^{1,2,3}$ \\
2 & $21.17 \pm 7.79^{3,4}$ & $20.09 \pm 6.73^{3,4}$ & $44.67 \pm 10.26^{1,2,4}$ & $55.85 \pm 3.69^{1,2,3}$ \\
3 & $16.42 \pm 4.89^{3,4}$ & $17.3 \pm 4.28^{3,4}$ & $34.61 \pm 4.92^{1,2}$ & $37.57 \pm 2.7^{1,2}$ \\
4 & $14.18 \pm 3.81^{3,4}$ & $14.96 \pm 2.73^{3,4}$ & $26.14 \pm 3.57^{1,2}$ & $28.71 \pm 1.09^{1,2}$ \\
5 & $11.54 \pm 2.96^{3,4}$ & $12.31 \pm 1.57^{3,4}$ & $21.39 \pm 2.37^{1,2}$ & $23.13 \pm 0.64^{1,2}$
\end{tabular}

Nota: letras en RSVP (L-RSVP), imágenes en RSVP (P-RSVP), letras en RCP (L-RCP) e imágenes en RCP (P-RCP). Las diferencias significativas $(p<0.05)$ han sido marcadas con un superíndice para señalar con qué condición ha sido diferente (1 para L-RSVP, 2 para P-RSVP, 3 para L-RCP, y 4 para P-RCP). Ha sido aplicada la corrección de Bonferroni.

La mejor puntuación en ITR del modo RCP frente al RSVP puede ser debido a dos factores: i) la velocidad de la presentación de las secuencias y ii) la mejor accuracy proporcionada por 
las interfaces RCP (como se ha mostrado arriba). A pesar de que el factor estímulo se ha mostrado también como un efecto principal, esto debe ser interpretado con cautela, ya que las diferencias entre las condiciones RSVP han sido inexistentes, mostrándose esta diferencia de forma clara para las condiciones bajo RCP.

\subsection{Tarea de escritura en línea}

Accuracy. Fue realizado un ANOVA $(2 \times 2)$ de medidas repetidas con los siguientes factores: presentación (RSVP y RCP) y estímulo (letras e imágenes) (Figura 2). El análisis no mostró ningún efecto principal ni de interacción. Sin embargo, cabe señalar que el efecto de interacción presentación $\times$ estímulo estuvo cerca de ofrecer un resultado significativo $(F(1$, 14) $=4,295 ; p=0,057$.

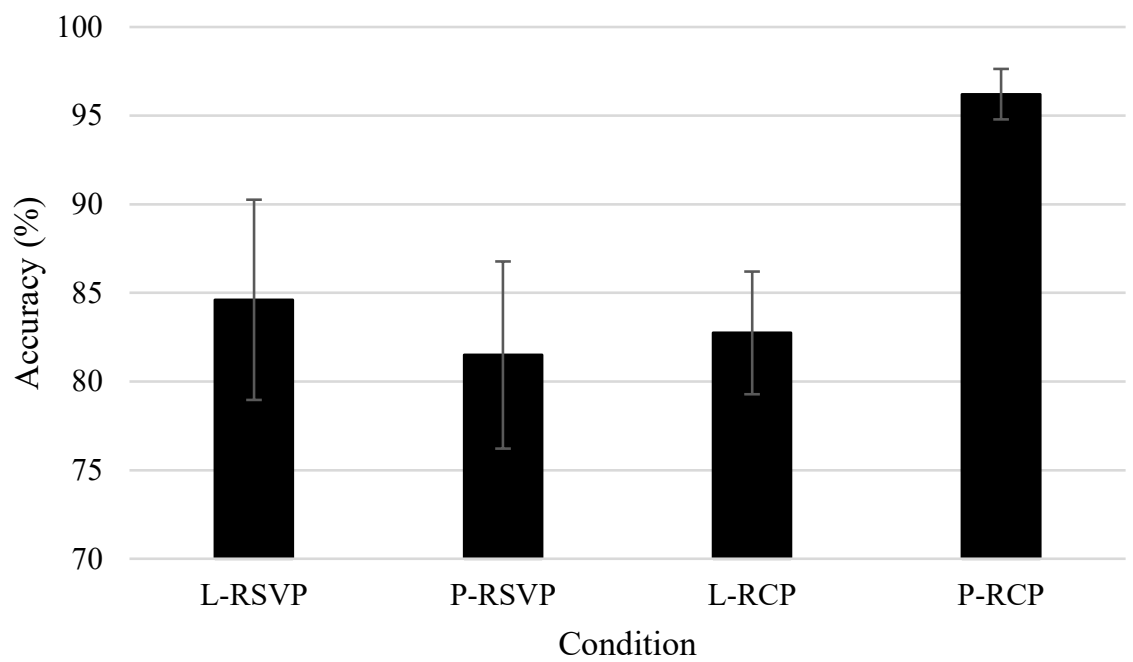

Fig. 2 Accuracy (\%, media \pm desviación estándar) en las diferentes condiciones: letras en RSVP (L-RSVP), imágenes en RSVP (P-RSVP), letras en RCP (L-RCP) e imágenes en RCP $(P-R C P)$.

A pesar de lo esperado, la accuracy en la tarea en línea no ha mostrado ninguna diferencia significativa. Sin embargo, estos resultados pueden explicarse por el criterio seleccionado para elegir el número de secuencias. Este criterio se basó en los resultados de la tarea de calibración, en la que se mostró un efecto techo: la mayoría de los participantes alcanzaban fácilmente el 100\% de accuracy. Por tanto, el clasificador tuvo que trabajar con una accuracy similar entre usuarios para la tarea de escritura (alrededor del 100\%), con la diferencia de que unas condiciones tardarían más que otras en hacer cada selección (ya que las condiciones obtuvieron la máxima accuracy con un número diferente de secuencias). 
Posible efecto de interacción entre el paradigma de presentación y el conjunto de estímulos en un teclado virtual controlado a través de una interfaz cerebro-ordenador basada en P300

Según la revisión sistemática realizada por Rezeika et al. (2018), el presente trabajo ha presentado niveles de accuracy, excepto los de la condición P-RCP, que han sido superiores a lo que se ofrece normalmente por otras propuestas. Sin embargo, debido al reducido número de comandos permitidos por nuestra propuesta- que fue seleccionado para establecer una comparación con el mismo número de estímulos en RCP y RSVP sin fatigar al usuario - y los criterios de selección de secuencias para la fase de escritura en línea, una comparación adecuada entre los estudios no es tarea fácil. Como alternativa, a diferencia de la accuracy, el ITR permite un mejor control de las siguientes variables para ofrecer una comparación más justa entre interfaces: número de elementos disponibles, accuracy y velocidad de escritura (ver la fórmula de Wolpaw, Ramoser, McFarland, y Pfurtscheller (1998) para más detalles).

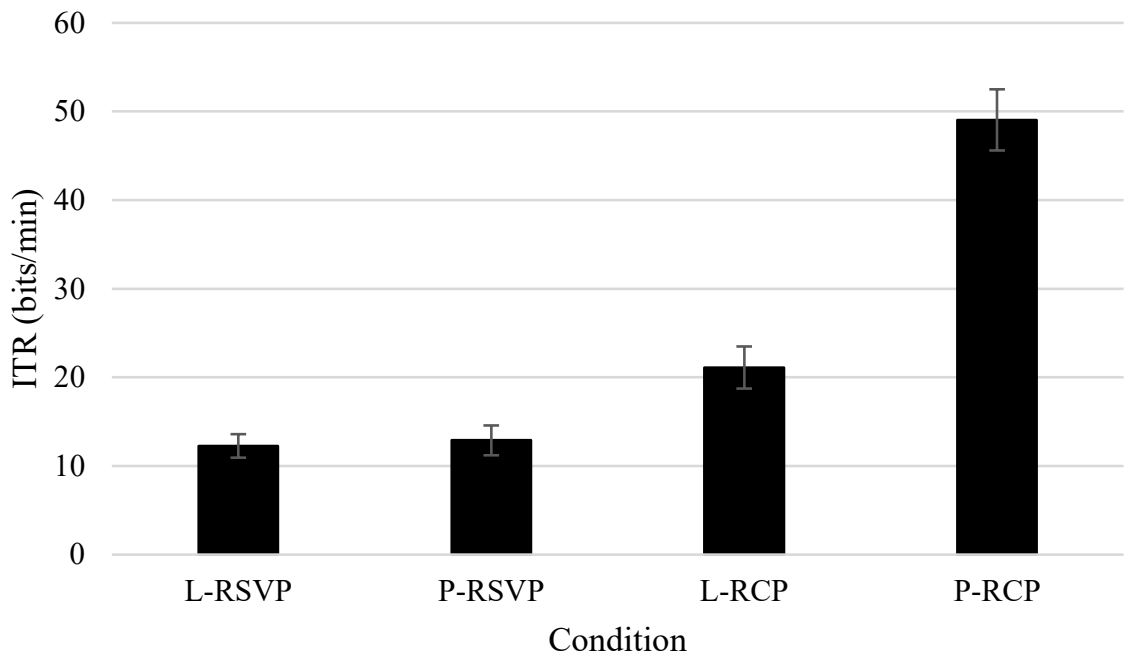

Fig. 3 Ratio de transferencia de información (bits/min, media \pm desviación estándar) en las diferentes condiciones: letras en RSVP (L-RSVP), imágenes en RSVP (P-RSVP), letras en RCP $(L-R C P)$ e imágenes en RCP $(P-R C P)$.

ITR. Fue realizado un ANOVA $(2 \times 2)$ de medidas repetidas con los siguientes factores: presentación (RSVP y RCP) y estímulo (letras e imágenes). El análisis mostró un efecto principal para los factores presentación $(F(1,14)=107,809, p<0,001)$ y estímulo $(F(1,14)$ $=90,215 ; p<0,001$. Por tanto, se podría afirmar que el modo de presentación $\operatorname{RCP}(35,08 \pm$ $18,37 \mathrm{bits} / \mathrm{min})$ y las imágenes fotos $(31,55 \pm 21,27 \mathrm{bits} / \mathrm{min})$ ofrecieron un ITR significativamente mayor que el modo RSVP $(12.58 \pm 5,77 \mathrm{bits} / \mathrm{min})$ y las letras $(16.83 \pm$ $8,81 \mathrm{bits} / \mathrm{min})$, respectivamente. Además, el efecto de interacción presentación $\times$ estímulo ofreció resultados significativos $(\mathrm{F}(1,14)=42.468, \mathrm{p}<.001)$, lo que sugiere que el efecto del estímulo depende de la presentación utilizada. Específicamente, la figura 3 muestra cómo el efecto de imágenes es mayor en RCP que en RSVP. Al igual que ocurrió para el ITR de la 
tarea de calibración, el efecto principal del estímulo debe ser interpretado con cautela, ya que las diferencias entre las condiciones RSVP son mínimas para las letras $(12,27 \pm 5,13)$ y para las imágenes $(12,89 \pm 6,52)$. Por el contrario, para las condiciones bajo RCP, las diferencias entre las letras y las imágenes fueron notables $(21,11 \pm 9,51$ y 49,05 $\pm 13,8$, respectivamente).

La condición P-RCP ha superado incluso el ITR en línea ofrecido por Li et al., (Li et al., 2015) en su propuesta de las caras verdes, que obtuvieron un promedio de $39 \pm 5 \mathrm{bits} / \mathrm{min}$. Por lo tanto, se puede afirmar que las imágenes podrían ser estímulos tan buenos como las caras verdes en un speller basado en P300. Sin embargo, hay otras propuestas que - mediante el uso de paradigmas más complejos que el modo RCP clásico - han conseguido mostrar mejores resultados en ITR (p. ej., Kaufmann y Kübler (2014; Yeom, Fazli, Ller, y Lee (2014)). Por tanto, podría ser interesante adaptar el uso de estas imágenes a los paradigmas de dicha presentación.

\section{Conclusiones}

El presente trabajo ha demostrado un fuerte efecto de interacción entre el paradigma de presentación y los estímulos en un speller basado en P300 visual. Este hallazgo es especialmente relevante si consideramos que la mayoría de investigaciones se han enfocado en el modo RCP, el cual requiere control ocular, una habilidad que la población objetivo de estos dispositivos puede tener afectada. Por lo tanto, sería conveniente que futuros trabajos pusieran a prueba anteriores propuesta hechas bajo RCP en otros paradigmas más convenientes para los pacientes, como el RSVP.

Asimismo, sería importante llevar a cabo estas propuestas con un mayor número de estímulos, en lugar de sólo nueve. Sin embargo, se destaca la posibilidad de utilizar matrices reducidas (p. ej., Ron-Angevin, Varona-Moya, y Silva-Sauer (2015)), ya que tienden a ofrecer un mejor rendimiento en accuracy (Sellers et al., 2006) y podrían ser un objetivo más realista para los futuros pacientes. Se debe recordar que, hasta la fecha, ningún BCI basado en P300 visual ha sido controlada por el paciente en CLIS (Guger et al., 2017). Además, el beneficio de una gran cantidad de elementos disponibles para la obtención de un buen ITR podría no ser suficiente para compensar la pérdida en accuracy, como puede verse en la sección de discusión de este artículo cuando se compara con otros trabajos.

En definitiva, además de para validar el uso de imágenes en un speller BCI basado en P300 visual, este estudio ha demostrado que no todos los estímulos ofrecen el mismo rendimiento bajo los modos de presentación RCP y RSVP. Por tanto, el presente trabajo muestra la importancia de probar los hallazgos realizados en RCP, relativos a los estímulos, en otros modos de presentación. 
Posible efecto de interacción entre el paradigma de presentación y el conjunto de estímulos en un teclado virtual controlado a través de una interfaz cerebro-ordenador basada en P300

Agradecimientos. Este trabajo fue parcialmente financiado por el proyecto SICCAU (RTI2018-100912-B-I00, MCIU/AEI/FEDER, UE) y la Universidad de Málaga. Por otra parte, los autores desean dar las gracias a todos los participantes por su colaboración.

\section{Referencias}

Bauer, G., Gerstenbrand, F., y Rumpl, E. (1979). Varieties of the locked-in syndrome. Journal of Neurology, 221(2), 77-91. https://doi.org/10.1007/BF00313105

Birbaumer, N. (2006). Breaking the silence: Brain-computer interfaces (BCI) for communication and motor control. Psychophysiology, 43(6), 517-532. https://doi.org/10.1111/j.14698986.2006.00456.x

Brunner, P., Joshi, S., Briskin, S., Wolpaw, J. R., Bischof, H., y Schalk, G. (2010). Does the "P300" speller depend on eye gaze? Journal of Neural Engineering, 7(5), 056013. https://doi.org/10.1088/1741-2560/7/5/056013

Corralejo, R., Hornero, R., y Álvarez, D. (2011). A domotic control system using Brain-Computer Interface (BCI). Lecture Notes in Computer Science (including subseries Lecture Notes in Artificial Intelligence and Lecture Notes in Bioinformatics) (Vol. 6691 LNCS). https://doi.org/10.1007/978-3-642-21501-8_43

Farwell, L. A., y Donchin, E. (1988). Talking off the top of your head: toward a mental prosthesis utilizing event-related brain potentials. Electroencephalography and Clinical Neurophysiology, 70(6), 510-523. https://doi.org/10.1016/0013-4694(88)90149-6

Fernández-Rodríguez, Á., Velasco-Álvarez, F., y Ron-Angevin, R. (2018). Evaluation of a P300 BrainComputer Interface Using Different Sets of Flashing Stimuli. In R. Ron-angevin (Ed.), BRAININFO 2018: The Third International Conference on Neuroscience and Cognitive Brain Information (pp. 1-4). Venice: IARIA.

Fuchino, Y., Nagao, M., Katura, T., Bando, M., Naito, M., Maki, A., ... Yoro, T. (2008). High cognitive function of an ALS patient in the totally locked-in state. Neuroscience Letters, 435(2), 85-89. https://doi.org/10.1016/j.neulet.2008.01.046

Guger, C., Spataro, R., Allison, B. Z., Heilinger, A., Ortner, R., Cho, W., y La Bella, V. (2017). Complete locked-in and locked-in patients: Command following assessment and communication with vibro-tactile P300 and motor imagery brain-computer interface tools. Frontiers in Neuroscience, 11(MAY), 251. https://doi.org/10.3389/fnins.2017.00251

Jones, M. R., y Sellers, E. W. (2019). Faces, locations, and tools: a proposed two-stimulus P300 brain computer interface. Journal of Neural Engineering, 1-17. https://doi.org/10.1088/17412552/aaff22

Kaufmann, T., y Kübler, A. (2014). Beyond maximum speed - A novel two-stimulus paradigm for brain-computer interfaces based on event-related potentials (P300-BCI). Journal of Neural Engineering, 11(5). https://doi.org/10.1088/1741-2560/11/5/056004 
Kellicut-Jones, M. R., y Sellers, E. W. (2018). P300 brain-computer interface: comparing faces to size matched non-face stimuli. Brain-Computer Interfaces, 5(1), 30-39. https://doi.org/10.1080/2326263X.2018.1433776

Lang, P. J., Bradley, M. M., y Cuthbert, B. N. (2008). International affective picture system (IAPS): Affective ratings of pictures and instruction manual. Technical Report A-8. University of Florida, Gainesville, FL. Technical Report A-8.

Lemoignan, J., y Ells, C. (2010). Amyotrophic lateral sclerosis and assisted ventilation: How patients decide. Palliative and Supportive Care, 8(2), 207-213. https://doi.org/10.1017/S1478951510000027

Li, Q., Liu, S., Li, J., y Bai, O. (2015). Use of a green familiar faces paradigm improves P300-speller brain-computer interface performance. PLoS ONE, 10(6), 1-15. https://doi.org/10.1371/journal.pone.0130325

Murguialday, A. R., Hill, J., Bensch, M., Martens, S., Halder, S., Nijboer, F., ... Gharabaghi, A. (2011). Transition from the locked in to the completely locked-in state: A physiological analysis. Clinical Neurophysiology, 122(5), 925-933. https://doi.org/10.1016/j.clinph.2010.08.019

Patterson, J. R., y Grabois, M. (1986). Locked-in syndrome: A review of 139 cases. Stroke, 17(4), 758 764. https://doi.org/10.1161/01.STR.17.4.758

Rezeika, A., Benda, M., Stawicki, P., Gembler, F., Saboor, A., y Volosyak, I. (2018). Brain-computer interface spellers: A review. Brain Sciences, 8(4). https://doi.org/10.3390/brainsci8040057

Ron-Angevin, R., Varona-Moya, S., y Silva-Sauer, L. Da. (2015). Initial test of a T9-like P300-based speller by an ALS patient. Journal of Neural Engineering, 12(4), 046023. https://doi.org/10.1088/1741-2560/12/4/046023

Sato, H., y Washizawa, Y. (2016). An N100-P300 Spelling Brain-Computer Interface with Detection of Intentional Control. Computers, 5(4), 31. https://doi.org/10.3390/computers5040031

Schalk, G., McFarland, D. J., Hinterberger, T., Birbaumer, N., y Wolpaw, J. R. (2004). BCI2000: A general-purpose brain-computer interface (BCI) system. IEEE Transactions on Biomedical Engineering. https://doi.org/10.1109/TBME.2004.827072

Sellers, E. W., Krusienski, D. J., McFarland, D. J., Vaughan, T. M., y Wolpaw, J. R. (2006). A P300 event-related potential brain-computer interface $(\mathrm{BCI})$ : The effects of matrix size and inter stimulus interval on performance. Biological Psychology, 73(3), 242-252. https://doi.org/10.1016/j.biopsycho.2006.04.007

Sutton, S., Braren, M., Zubin, J., y John, E. R. (1965). Evoked-potential correlates of stimulus uncertainty. Science. https://doi.org/10.1126/science.150.3700.1187

Townsend, G., LaPallo, B. K., Boulay, C. B., Krusienski, D. J., Frye, G. E., Hauser, C. K., ... Sellers, E. W. (2010). A novel P300-based brain-computer interface stimulus presentation paradigm: Moving beyond rows and columns. Clinical Neurophysiology, 121(7), 1109-1120. https://doi.org/10.1016/j.clinph.2010.01.030

Treder, M. S., y Blankertz, B. (2010). (C)overt attention and visual speller design in an ERP-based 
Posible efecto de interacción entre el paradigma de presentación y el conjunto de estímulos en un teclado virtual controlado a través de una interfaz cerebro-ordenador basada en P300

brain-computer interface. Behavioral and Brain Functions, 6. https://doi.org/10.1186/17449081-6-28

Velasco-Álvarez, F., Sancha-Ros, S., García-Garaluz, E., Fernández-Rodríguez, Á., Medina-Juliá, M. T., y Ron-Angevin, R. (2019). UMA-BCI Speller: an Easily Configurable P300 Speller Tool for End Users. Computer Methods and Programs in Biomedicine, 172, 127-138. https://doi.org/10.1016/J.CMPB.2019.02.015

Wolpaw, J. R., Ramoser, H., McFarland, D. J., y Pfurtscheller, G. (1998). EEG-based communication: Improved accuracy by response verification. IEEE Transactions on Rehabilitation Engineering, 6(3), 326-333. https://doi.org/10.1109/86.712231

Wolpaw, J. R., Birbaumer, N., McFarland, D. J., Pfurtscheller, G., y Vaughan, T. M. (2002). Braincomputer interfaces for communication and control. Clinical Neurophysiology, 113(6), 767-791. https://doi.org/10.1016/S1388-2457(02)00057-3

Yeom, S. K., Fazli, S., Ller, K. R. M., y Lee, S. W. (2014). An efficient ERP-based brain-computer interface using random set presentation and face familiarity. PLOS ONE, 9(11), 1-13. https://doi.org/10.1371/journal.pone.0111157

Zhang, R., Wang, Q., Li, K., He, S., Qin, S., Feng, Z., ... Li, Y. (2017). A BCI-based environmental control system for patients with severe spinal cord injuries. IEEE Transactions on Biomedical Engineering. https://doi.org/10.1109/TBME.2016.2628861 


\title{
Estudio preliminar de la usabilidad de tres tamaños de teclados basados en Interfaces Cerebro-Computador por pacientes con ELA
}

\section{Medina-Juliá, M. Teresa; Fernández-Rodríguez, Álvaro; Velasco-Álvarez, Francisco y Ron-Angevin, Ricardo}

Departamento de Tecnología Electrónica, Universidad de Málaga, Málaga, España. maytemed@uma.es, afernandezrguez@uma.es, fvelasco@uma.es,rron@uma.es

\begin{abstract}
Brain-Computer Interface-based spellers which use the P300 bio-potential as control signal have been widely studied. Nevertheless, the optimal speller size has not been properly presented for motor-disabled people. In the present work tree different speller sizes were assessed through the usability approach - in terms of effectiveness, efficiency and satisfaction - to offer a proper one. Four patients diagnosed with amyotrophic lateral sclerosis evaluated the speller sizes. They achieved worse performance with the small size, especially in the effectiveness and satisfaction dimensions; while with the medium size they achieved better performance, highlighting satisfaction. The results show that the speller size must be considered while designing a new paradigm for motor-disabled.
\end{abstract}

Keywords: Amyotrophic Lateral Sclerosis (ALS), brain-computer interface (BCI), speller, P300, speller size, usability.

\begin{abstract}
Resumen
Con referencia a los teclados basados en Interfaces Cerebro Computador que utilizan el biopotencial P300 como señal de control, el tamaño óptimo para personas con discapacidad motora no se ha presentado propiamente. En este trabajo se evaluaron tres tamaños de teclados mediante el enfoque de usabilidad - en términos de efectividad, eficiencia y satisfacción - para ofrecer uno adecuado. Cuatro pacientes con esclerosis lateral amiotrófica evaluaron los tres tamaños. Los participantes tuvieron peor desempeño con el tamaño pequeño, especialmente en la efectividad y satisfacción; mientras que con el mediano obtuvieron mejores resultados, resaltando la satisfacción. Los resultados muestran que se debe considerar el tamaño del teclado al diseñar un nuevo paradigma para personas con discapacidad.
\end{abstract}

Palabras clave: Esclerosis lateral amiotrófica (ELA), Interfaces CerebroComputador (BCI), teclado, P300, tamaño de teclado, usabilidad. 
Estudio preliminar de la usabilidad de tres tamaños de teclados basados en Interfaces CerebroComputador por pacientes con ELA

\section{Introducción}

La Esclerosis Lateral Amiotrófica (ELA) es un desorden neurológico que degenera las neuronas motoras periféricas y centrales, llevando hasta la parálisis total del paciente (Patterson y Grabois, 1986). Sin embargo, se preservan otras funciones como son la percepción sensitiva o las habilidades intelectuales.

Investigadores del campo han desarrollado diferentes sistemas para proporcionar a estos pacientes un canal alternativo de comunicación con su entorno, tales como sistemas eyetracker (Pal, Mangal, y Khosla, 2017), o Interfaces Cerebro Computador (BCI, del inglés Brain-Computer Interfaces) (Birbaumer, 2006). En fases evolucionadas de la enfermedad, los pacientes pueden perder parcial o totalmente el control ocular (imprescindible para controlar sistemas basados en eye-tracker). En estos casos los sistemas BCI sirven como última vía de comunicación para ellos, ya que permiten que los pacientes interactúen con su entorno sin necesidad de realizar movimientos motores, utilizando como señal de control la actividad cerebral del sujeto (Nicolas-Alonso y Gomez-Gil, 2012).

Los sistemas BCI utilizan la electroencefalografía (EEG) para medir la actividad cerebral del sujeto y clasificar las distintas formas de onda como son los ritmos sensoriomotores (SMR, del inglés Sensorimotor Rhythms), o potenciales evocados P300 (P300-EP, del inglés P300 Evoked Potentials).

Este trabajo se centra en los potenciales evocados P300, los cuales son unas variaciones positivas de amplitud de la señal cerebral que aparecen aproximadamente $300 \mathrm{~ms}$ después de que aparezca un estímulo extraño al que se está a la espera. Esta señal es normalmente utilizada por unos BCIs llamados teclados virtuales (Nicolas-Alonso y Gomez-Gil, 2012). Estos sistemas están formados por una matriz de letras, números, símbolos y/o comandos. El paradigma más comúnmente usado es el paradigma oddball propuesto por (Farwell y Donchin, 1988), donde cada columna y fila de la matriz se iluminan de manera pseudoaleatoria. El sujeto debe prestar atención a un elemento específico de la matriz mientras las filas y las columnas son iluminadas, y cuando su elemento objetivo se ilumina, el biopotencial eléctrico P300 es evocado en el cerebro y es reconocido por el sistema.

Anteriormente, se ha estudiado el efecto que producen diversas variaciones visuales de las matrices basadas en P300. Por ejemplo, los colores de los estímulos, la naturaleza de los mismos y el paradigma aplicado (Ikegami et al., 2012; Li et al., 2015; Acqualagna et al., 2013; Ron-Angevin et al., 2015). Las medidas del teclado se han estudiado en escasas ocasiones. En concreto, se ha estudiado el efecto que tienen distintas dimensiones de matrices (Sellers et al., 2006), el efecto que tiene el tamaño de los símbolos (Salvaris y Sepulveda, 2009), el tamaño del monitor en el que se presenta el teclado (Y. Li et al., 2011). Pero ninguno nos da información suficiente para determinar un tamaño óptimo de teclado en cuanto al tamaño de símbolos y distancia entre ellos. Por otra parte, (Ron- 
Angevin et al., 2019) propone en su trabajo un tamaño de teclado óptimo entendido desde el punto de vista de la usabilidad bajo dos condiciones opuestas de movilidad ocular (permitiendo dirigir la mirada al símbolo de interés y manteniendo la mirada en el centro del teclado). El trabajo anterior concluyó que el teclado mediano es el que mejores prestaciones presenta en cuanto al rendimiento (empatado en esta dimensión con el pequeño) y la satisfacción.

Es importante resaltar que todos los artículos arriba mencionados realizaron su estudio con participantes sanos. Por lo que es importante constatar los resultados con los que son realmente los usuarios finales de este tipo de sistema. (Ron-Angevin et al., 2019) estudió una condición que podría ser traducida a pacientes con movilidad ocular reducida (i.e., la condición en la que se impedía mover la mirada del centro del teclado). Sin embargo, esta condición no es del todo realista pues los pacientes podrían conservar movimientos de cara y ojos residuales (Patterson y Grabois, 1986). Además, aprovechar estos movimientos residuales podría ser de gran ayuda para controlar el teclado pues, como (Ron-Angevin et al., 2019) y (Brunner et al., 2010) demostraron, el rendimiento de estos sistemas aumenta significativamente cuando se dirige la mirada al símbolo de interés. Por ello se hace necesario encontrar el tamaño de teclado que, en general, sea usable para los pacientes. Tamaños grandes de teclado podrían encontrarse difícil de manejar no sólo por rango de movimiento ocular limitado sino también por rango de movimiento de cara y cuello limitados. Por otra parte, un teclado muy pequeño podría causar problemas cuando un usuario intente percibir correctamente los diferentes elementos de la matriz, pero podría resultar menos cansado físicamente.

Por tanto, el objetivo del presente trabajo es evaluar de manera preliminar el efecto que provocan en pacientes, diagnosticados con ELA, los tres tamaños de teclados probados por (Ron-Angevin et al., 2019). Los resultados de los participantes fueron analizados individualmente para buscar tendencias relacionas con cualquiera de los teclados. Al igual que (Ron-Angevin et al., 2019), se empleó el modelo de usabilidad según la ISO (2000) para la evaluación; por tanto, tres factores fueron estudiados: efectividad, eficiencia y satisfacción.

\section{Método}

\subsection{Participantes}

Cuatro pacientes españoles diagnosticados con ELA (P1-P4, todos hombres, edad $64.5 \pm$ 6.95) participaron en el presente estudio. Cabe mencionar que el quinto participante con ELA (P5) no pudo participar en el experimento ya que tenía escaso control del parpadeo por lo que tenía problemas para mantener sus ojos abiertos. Todos los participantes (o su correspondiente cuidador) dieron su consentimiento informado. 
Estudio preliminar de la usabilidad de tres tamaños de teclados basados en Interfaces CerebroComputador por pacientes con ELA

Según lo declarado por los participantes, ninguno tenía historial de alguna enfermedad neurológica - además de ELA - o psiquiátrica, y tenían visión normal o corregida. Ninguno de ellos tenía experiencia previa con sistemas BCI. Se contactó con los pacientes a través de la Asociación de ELA de Andalucía. Las pruebas tuvieron lugar en sus respectivas casas. En la Tabla 1 se proporciona información sobre los pacientes. Este estudio fue aprobado por el Comité de Ética de la Universidad de Málaga y cumplió los estándares de ética de la Declaración de Helsinki.

Tabla 1. Información sobre los participantes de este experimento.

\begin{tabular}{ccccc}
\hline Participante & $\begin{array}{c}\text { Edad } \\
\text { (años) }\end{array}$ & $\begin{array}{c}\text { Años post- } \\
\text { diagnóstico }\end{array}$ & ALSFRS-R & $\begin{array}{c}\text { Canal de comunicación } \\
\text { habitual }\end{array}$ \\
\hline P1 & 64 & 4 & 12 & Voz \\
P2 & 73 & 5 & 29 & Manos \\
P3 & 56 & 4 & 0 & Ojos (mirada y pestañeos) \\
P4 & 65 & 20 & 19 & Voz \\
\hline
\end{tabular}

\subsection{Registro y procesado de señales EEG}

Para registrar las señales se utilizó el amplificador Acti-Champ (Brain Products GmbH, Munich, Germany). Las posiciones utilizadas, según el sistema internacional 10/20, fueron Fz, Cz, Pz, Oz, P3, P4, PO7 y PO8, utilizando como referencia la posición TP6 y como tierra AFz. Se controló que la impedancia de la señal se mantuviera $<10 \mathrm{k} \Omega$. La señal se filtró utilizando un paso banda de $0.1-30 \mathrm{~Hz}$. Se utilizó BCI2000 para controlar todos los aspectos de la colección y procesado de los datos.

\subsection{Paradigmas de presentación}

Los tres teclados fueron diseñados acorde a las especificaciones dadas por (Ron-Angevin et al., 2019), donde los tres tamaños tenían una apariencia similar al teclado P300 clásico. Los teclados consistieron en un matriz de caracteres de $6 \times 6$ con el alfabeto inglés y con los números del 0 al 9 (Figura 1). La duración de un flash y el tiempo inter simbólico (ISI) fueron de $128 \mathrm{~ms}$. Después de cada secuencia de flashes había una pausa de $2 \mathrm{~s}$ excepto para el paciente $\mathrm{P} 4$ que utilizó 6s debido a un error en la aplicación del protocolo. En las fases de calibración y escritura libre se utilizaron 10 secuencias. Importante notar que una secuencia consiste en iluminar una vez cada columna y cada fila, por lo que cada carácter es estimulado dos veces por cada secuencia.

Las medidas de los teclados se muestran en la Tabla 2 de acuerdo a los parámetros establecidos en la Figura 1. 


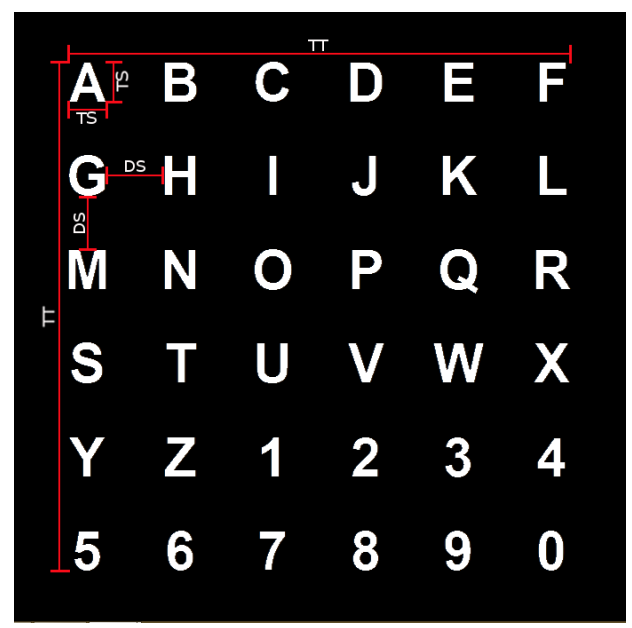

Fig. 1 Tipografia de teclado utilizado. Además, se señalan las medidas consideradas en el presente trabajo. Donde 'TT' simboliza tamaño de teclado, 'TS' tamaño de símbolo y 'DS' distancia entre símbolos.

Tabla 2. Valores de las medidas por tamaño de teclado.

\begin{tabular}{cccc}
\hline \multirow{2}{*}{ Tamaño } & \multicolumn{3}{c}{ Parameter (cm) } \\
\cline { 2 - 4 } & $\begin{array}{c}\text { Tamaño de } \\
\text { teclado (TT) }\end{array}$ & $\begin{array}{c}\text { Tamaño de } \\
\text { símbolo (TS) }\end{array}$ & $\begin{array}{c}\text { Distancia entre } \\
\text { símbolos (DS) }\end{array}$ \\
\hline Pequeño & 5.27 & 0.42 & 0.55 \\
Mediano & 9.98 & 0.79 & 1.04 \\
Grande & 14.69 & 1.17 & 1.53 \\
\hline
\end{tabular}

\subsection{Procedimiento}

En cuanto al procedimiento, se siguió lo indicado por (Ron-Angevin et al., 2019). El experimento consistió en tres sesiones (una por cada teclado). El orden de los teclados fue contrabalanceado para controlar efectos no deseados como la experiencia. En cuanto al tiempo entre sesión y sesión se estableció un rango entre 5 horas y 3 días. En cada sesión se llevaron a cabo las siguientes acciones: i) realizar una tarea de calibración, ii) realizar una tarea de escritura libre y, finalmente, iii) rellenar unos cuestionarios subjetivos referentes al teclado que habían controlado en dicha sesión. En concreto, cada tarea consistió en:

- Tarea de calibración: las palabras a calibrar fueron 'LUNA', 'RAMO', 'KILO' y '2015'. Esta fase tomó aproximadamente 10 minutos.

- Tarea de escritura libre: en esta fase, se pidió a los participantes que deletrearan las palabras 'CHAT', 'PURE' y '1935'. En caso de que el clasificador eligiera una letra errónea, se les indicó que pasaran a la siguiente letra sin posibilidad de corregir el error. 
Estudio preliminar de la usabilidad de tres tamaños de teclados basados en Interfaces CerebroComputador por pacientes con ELA

- Cuestionarios subjetivos: la última parte de cada sesión consistió en contestar dos cuestionario diferentes i) la escala analógica visual (VAS, del inglés visual analogue scale) y ii) el test NASA-TLX. Por último, cuando se completaban las tres sesiones, se debía rellenar un cuestionario comparativo de los tres teclados.

Solo las últimas tres palabras calibradas se utilizaron para la clasificación. El clasificador utilizado fue un Stepwise Linear Discriminant Analysis (SWLDA).

\subsection{Evaluación de la usabilidad}

La evaluación de la usabilidad se llevó a cabo considerando la definición propuesta por ISO (2000), la cual incluye tres dimensiones: efectividad, eficiencia y satisfacción.

\subsubsection{Efectividad}

La efectividad está relacionada con la precisión con la que el usuario completó la tarea. Para esta dimensión se tuvieron en cuenta i) la precisión en la tarea de calibración, y ii) el error de clasificación obtenido en la tarea de escritura libre.

\subsubsection{Eficiencia}

La eficiencia está relacionada con los recursos gastados para completar la tarea. Para evaluarla, se han considerado dos resultados: i) la carga de trabajo subjetiva utilizando el NASA-TLX, ii) el cuestionario VAS sobre preguntas relacionadas con características de los teclados. Cada uno de éstos evalúa los siguientes parámetros:

- $\quad$ NASA-TLX: evaluó la demanda mental, física y temporal, así como el desempeño, el esfuerzo y la frustración percibida por el participante. La predominancia de cada uno de estos parámetros en la carga de trabajo total es computada en escala del 0-100 como media de pesos, donde la puntuación máxima de cada parámetro es 33.33.

- VAS: se ponderan cada uno de los parámetros de 0-10 (donde 0 es el mínimo y 10 el máximo). Lo que evaluó fue: i) la dificultad en percibir los caracteres alejados del centro, y ii) la dificultad en distinguir las diferentes columnas y filas.

\subsubsection{Satisfacción}

Finalmente, la satisfacción está relacionada con la actitud de los usuarios. La preferencia y lo sentido por el usuario fue analizado utilizando un cuestionario comparativo basado en la Escala de la Usabilidad del Sistema (SUS) (Brooke, 1996). En este cuestionario se les pidió 
a los usuarios que puntuaran por cada teclado el nivel de complejidad, estrés causado, sensación de control, cansancio, y preferencia.

\section{Resultados y discusión}

En esta sección los resultados de cada paciente son presentados individualmente ya que no fue posible realizar un estudio estadístico por el bajo tamaño muestral. Al mismo tiempo que se presentan los resultados se irán discutiendo y comparando con la literatura.

\subsection{Efectividad}

En este apartado se muestran los resultados en apartados distintos según la precisión alcanzada en la fase de calibración y el error obtenido en la fase de escritura libre.

\subsubsection{Precisión obtenida en la fase de calibración}

Se ha realizado un análisis estadístico de caso único para cada uno de los participantes. La precisión alcanzada por cada participante se puede observar en la Figura 2.

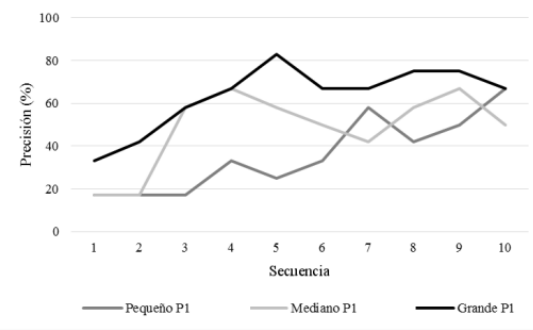

a)

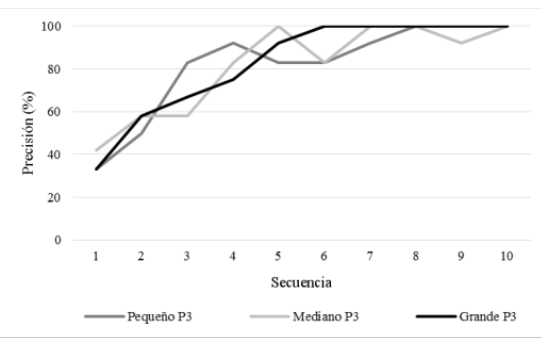

c)

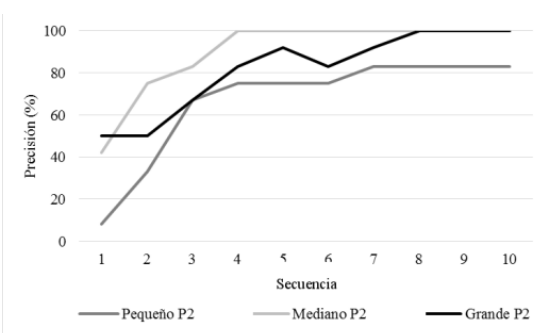

b)

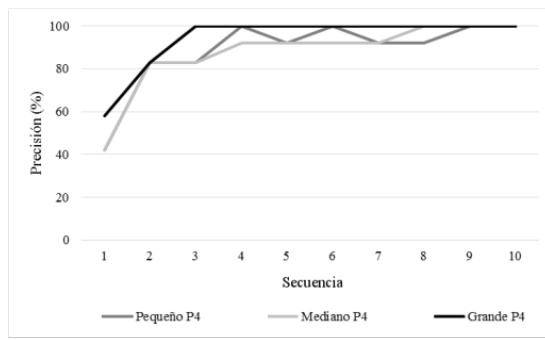

d)

Fig. 2 Resultados de la fase de calibración de los cuatro participantes al utilizar los tres tamaños de teclado: a) corresponde a los resultados del participante P1, b) a los del participante P2, c) a los del P3, yd) a los del P4. 
Estudio preliminar de la usabilidad de tres tamaños de teclados basados en Interfaces CerebroComputador por pacientes con ELA

A continuación se detallan los resultados de cada uno de los participantes:

- Participante P1: este participante obtuvo una precisión relativamente baja durante esta fase con los tres tamaños: pequeño, $35.9 \pm 17.95 \%$; mediano, $48.4 \pm 18.22 \%$; y grande, $63.4 \pm 15.33 \%$. Se obtuvieron diferencias significativas con respecto al teclado utilizado $(F(2,18)=14.573 ; p<.001)$. En concreto, el teclado grande obtuvo una precisión significativamente mejor que la de los teclados mediano y pequeño ( $p=.002$, en ambos casos). Aunque no se obtuvieron diferencias significativas entre el teclado pequeño y mediano, mirando los resultados obtuvo peor desempeño con el teclado pequeño (Figura 2.a).

- Participante P2: sus resultados fueron: pequeño $66.5 \pm 25.5 \%$, mediano $90 \pm 19.1 \%$ y grande $81.7 \pm 19.57 \%$. Se obtuvieron diferencias significativas con respecto al teclado $(F(2,18)=27.847 ; p<.001)$. En concreto, el teclado pequeño obtuvo significativamente peor rendimiento que los teclados grande $(p=.006)$ y mediano $(p$ $<.001)$. Aunque no se obtuvieron resultados significativamente mejores, viendo la Figura 2.b, se observa que tuvo mejor rendimiento con el mediano.

- Participante P3: obtuvo como resultados: pequeño $81.6 \pm 22.6 \%$, mediano $81.6 \pm$ $21.47 \%$ y grande $82.5 \pm 23.5 \%$. En este caso no se obtuvieron diferencias significativas con respecto al tamaño de teclado utilizado. Mirando a la Figura 2.c, sus mejores resultados los obtuvo con los teclados mediano y grande.

- Participante P4: este sujeto fue el participante que consiguió los mejores resultados: para el tamaño pequeño $88.4 \pm 17.6 \%$, para el mediano $87.6 \pm 17.19 \%$ y el grande $94.1 \pm 13.76 \%$. Se obtuvieron diferencias significativas entre teclados $(F(2,18)=$ $6.95, p=.006)$. En concreto, la precisión del teclado grande fue significativamente mejor que la del teclado mediano $(p=.034)$.

Ninguno de los teclados puede ser considerado como el mejor, sin embargo, parece que el teclado grande ofrece mejores resultados por lo menos para dos de los participantes. De igual forma, se puede observar que el teclado pequeño es el que en general ha obtenido los peores resultados, dejando al mediano entre ambos teclados. Al existir diferencias individuales, se enfatiza la importancia de que el tamaño debe adaptarse a las características y preferencias del sujeto.

Observando el resultado obtenido en la décima secuencia, la mayoría los participantes consiguieron 100\%; en concreto, P3 y P4 con los tres teclados, y P2 con el grande y el mediano. En el caso de P1, no consiguió 100\% con ninguno de ellos. Esta información es importante pues esta secuencia es la que se eligió como parámetro fijo para la fase online. Cabe resaltar que P2 y P3 consiguieron su precisión más alta con menos número de secuencias con el teclado mediano (en secuencia 4 y 5 , respectivamente), mientras que P1 y P4 con el teclado grande (en las secuencias 4 y 5 , respectivamente) considerando que P1 no alcanzó $100 \%$. Por lo tanto, para todos los sujetos, el teclado pequeño es el menos 
recomendado. Comparando el resultados de estos sujetos con los obtenidos por (RonAngevin et al., 2019), tanto los participantes de este experimento como los sujetos sanos consiguieron sus puntuaciones máximas en el mismo rango de secuencias (i.e., entre 4 y 6 ). Estos resultados también concuerdan con los obtenidos por (Ryan et al., 2018) por otros participantes con ELA que controlaron una matriz de $6 \times 6$, pues obtuvo que los participantes necesitaron entre 4 y 7 secuencias para alcanzar su puntuación máxima.

\subsubsection{Error obtenido en la fase de escritura libre}

La Tabla 3 muestra el error obtenido en la fase de escritura libre para los cuatro participantes. El error se calculó dividendo el número de errores entre los 12 caracteres a deletrear.

Tabla 3. Porcentaje de error obtenido en la tarea de escritura libre. El error se calculó dividiendo el número de errores por el número total de caracteres deletreados.

\begin{tabular}{cccc}
\hline \multirow{2}{*}{ Participante } & \multicolumn{3}{c}{ Tamaño de teclado } \\
\cline { 2 - 4 } & Pequeño & Mediano & Grande \\
\hline P1 & 83.33 & 41.67 & 58.33 \\
P2 & 33.33 & 25 & 25 \\
P3 & 0 & 0 & 16.67 \\
P4 & 8.33 & 8.33 & 0 \\
\hline
\end{tabular}

A continuación, se detallan los resultados obtenidos por cada uno de los participantes:

- Participante P1: para P1 el mejor teclado fue el mediano, consiguiendo un error de $41.667 \%$ y el peor el pequeño con un error del $83.33 \%$.

- Participante P2: este participante obtuvo el mismo resultado con los teclados grande y mediano (25\% de error), y con el pequeño obtuvo su peor resultado $(33.33 \%)$.

- Participante P3: este participante obtuvo el mismo porcentaje de error con el teclado pequeño y mediano ( $0 \%$ ), mientras que con el grande obtuvo un $16.67 \%$ (lo que significa que sólo cometió dos errores).

- Participante P4. Este participante obtuvo su mejor resultado con el teclado grande $(0 \%)$. Con los otros dos obtuvo el mismo porcentaje de $8.33 \%$, lo que corresponde a un único error.

Comparando los resultados de los cuatro participantes con otros estudios con pacientes con ELA que han utilizado teclados basados en el de (Farwell y Donchin, 1988) y tamaños de matriz grande (ej., (McCane et al., 2014; Nijboer et al., 2008; Poletti et al., 2016)), se pude observar que los resultados obtenidos son representativos de la población. 
Estudio preliminar de la usabilidad de tres tamaños de teclados basados en Interfaces CerebroComputador por pacientes con ELA

El teclado mediano ofreció los mejores resultados en términos de error para P1 (46.67\%), P2 (25\%, junto con el tamaño grande) y P3 ( $0 \%$, junto con el pequeño). Por el contrario, el pequeño fue el que obtuvo los peores resultados para P1 (83.33\%), P2 (33.33\%) y P4 (8.33\%, junto con el tamaño mediano). Comparando los resultados de los pacientes entre ellos, P1 y P2 fueron los que peores resultados obtuvieron. Esto pudo ser debido a los $2 \mathrm{~s}$ de pausa entre letras configurado, ya que este tiempo podría no haber sido suficiente para ellos para dirigir la mirada al siguiente símbolo, lo cual pudo haber afectado su desempeño. Sin embargo, esta pausa se mantuvo en los tres teclados, por lo que esto podría no ser relevante para el estudio.

Por otra parte, si utilizamos el criterio de (Kubler et al., 2001) el cual indica que $30 \%$ es el error máximo permitido para establecer un sistema de comunicación eficiente, el teclado pequeño es usable solo por el 50\% de los participantes (P3 y P4), mientras que el mediano y grande para el 75\% de ellos (P2, P3 y P4 en ambos casos). Estos resultados indican que el pequeño puede ser el que aporte menos beneficios.

\subsection{Eficiencia}

En esta sección se muestran los resultados obtenidos en los cuestionarios subjetivos, donde cada uno de ellos (i.e., NASA-TLX y VAS) corresponden a un apartado distinto.

\subsubsection{NASA-TLX}

En la Figura 3 se muestran las puntuaciones ponderadas de los parámetros por sujeto.

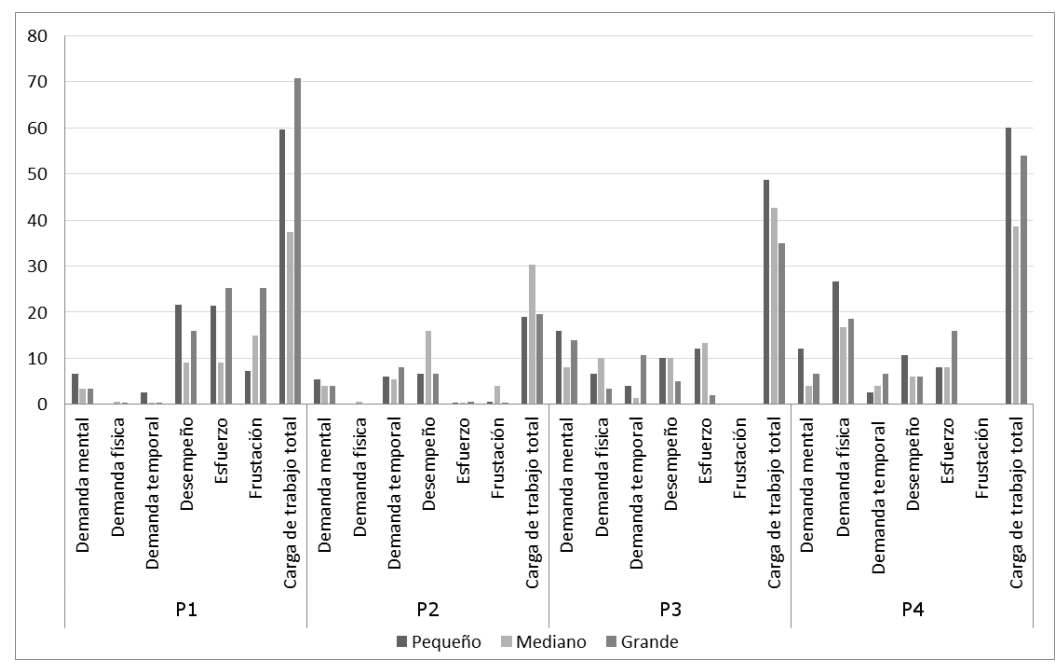

Fig. 3 Resultados obtenidos en el test NASA-TLX con sus parámetros ya ponderados. 
A continuación, se detallan los resultados por participante:

- Participante P1: puntuó con los valores más bajos al teclado mediano (carga de trabajo total, 37.33). Estas diferencias fueron sobretodo observables en el desempeño (puntuación ponderada, 9), esfuerzo (puntuación ponderada, 9), y frustración (puntuación ponderada, 15). Por otra parte, al teclado que puntuó con valores más altos fue al teclado grande (carga de trabajo, 70.67), especialmente remarcable en las dimensiones esfuerzo y frustración (ambas puntuadas ponderadamente con 25.33).

- Participante P2: este sujeto puntuó con los valores más altos al teclado mediano (carga de trabajo total, 30.33), mientras que a los otros dos los puntuó con valores más bajos muy cercanos (19 y 19.67 al pequeño y al grande, respectivamente). El mediano obtuvo principalmente una puntuación relativamente alta en la dimensión desempeño (puntuación ponderada, 16).

- Participante P3: es difícil encontrar tendencias claras con los resultados de este participante. Sin embargo, parece que la carga de trabajo aumenta conforme disminuye el tamaño del teclado.

- Participante P4. Con este participante la tendencia se ve clara. El teclado mediano obtuvo los valores más bajos (carga de trabajo total, 38.67), sobretodo en las variables demanda mental (puntuación ponderada, 4), física (puntuación ponderada, 16.67) y temporal (puntuación ponderada, 8). El tamaño que obtuvo las mayores puntuaciones fue el pequeño (carga de trabajo total, 60), resaltando la demanda mental y fisica (puntuación ponderada, 12 y 26.67, respectivamente) y el desempeño (puntuación ponderada, 10.68).

El trabajo realizado por (Pasqualotto et al., 2015), consiste en un estudio de la usabilidad de los teclados BCI con personas con ELA. En dicho estudio se obtuvo una carga total media de $47.64 \pm 14.87$, la cual engloba a los resultados obtenidos para el teclado grande de tres de los participantes del presente estudio, excepto P1, el cual declaró una carga de trabajo muy por encima de la media (70.67). En (Ron-Angevin et al., 2019), las medias de carga de trabajo para cada uno de los tamaños (pequeño: $40.4 \pm 7.2$, mediano: $38.22 \pm 4.8$, grande: $41.2 \pm 6.4$ ) engloban los resultados de los tres teclados de P3 y los de los teclados medianos de $\mathrm{P} 1$ y $\mathrm{P} 4$, mientras que son menores con respecto a los resultados de los teclados grande y pequeño de P1 y P4, y mayores con respecto a los tres de P2.

\subsection{2. $V A S$}

Esta sección presenta los resultados obtenidos en el cuestionario VAS sobre la percepción subjetiva (véase la Tabla 4 para ver los resultados de los participantes). 
Estudio preliminar de la usabilidad de tres tamaños de teclados basados en Interfaces CerebroComputador por pacientes con ELA

Tabla 4. Respuestas al cuestionario VAS.

\begin{tabular}{ccccccc}
\hline \multirow{2}{*}{ Participante } & \multicolumn{5}{c}{ Pregunta sobre percepción para cada tamaño } \\
\cline { 2 - 7 } & \multicolumn{4}{c}{ Q1 } & Q2 \\
\cline { 2 - 7 } & $\mathrm{P}$ & $\mathrm{M}$ & $\mathrm{G}$ & $\mathrm{P}$ & $\mathrm{M}$ & $\mathrm{G}$ \\
\hline P1 & 2 & 2 & 6 & 5 & 2 & 2 \\
P2 & 0 & 0 & 0 & 0 & 0 & 0 \\
P3 & 0 & 0 & 0 & 0 & 0 & 0 \\
P4 & 8 & 5 & 7 & 6 & 5 & 2 \\
\hline
\end{tabular}

Nota: Q1 simboliza la 'Dificultad para percibir los simbolos alejados del centro'; Q2 la 'Dificultad para distinguir entre las filas y las columnas'; $P$ representa 'pequeño', $M$ 'mediano' y $G$ 'grande'. La puntuación varía de 0 a $10(0=$ muy fácil; $10=$ muy dificil).

Por una parte, P1 y P3 declararon no haber tenido alguna dificultad en cuanto a las preguntas planteadas sobre los teclados. Nosotros pensamos que es debido a la actitud positiva y a la gran motivación que presentaron dichos participantes. Por otra parte, P1 y P4 coinciden en que el tamaño pequeño es el más complicado para distinguir entre las distintas filas y columnas $(Q 2)$. Mientras que la primera pregunta $(Q 1)$ está contrabalanceada en los otros teclados. Dados los resultados, parece complicado destacar tendencias de ellos.

Es interesante resaltar que $\mathrm{P} 4$ ha seleccionado puntuaciones parecidas a las obtenidas por los participantes sanos de (Ron-Angevin et al., 2019) (Q1: pequeño: $2.2 \pm 0.7$, mediano: 1.2 \pm 0.4 , grande: $1.8 \pm 0.5$; Q2: pequeño: $2.7 \pm 0.8$, mediano: $1.15 \pm 0.4$, grande: $1.6 \pm 0.5$ ) Puesto que este participante alcanzó puntuaciones parecidas o incluso mejores en la parte de eficiencia que la media de los participantes de dicho estudio. En cuanto a P1, la puntuación de $Q 1$ puede estar relacionada con la carga de trabajo total pues en ambos casos puntuó negativamente al teclado grande. Pensamos que ambas puntuaciones pueden estar relacionadas con su declarada dificultad para percibir todas las letras. Sin embargo, con el teclado grande y mediano fue con los que obtuvo mejores resultados en la parte de la eficiencia, lo cual puede ser debido a una mayor facilidad para distinguir las filas y las columnas (i.e., $Q 2)$.

\subsection{Satisfacción}

En la Figura 4 se puede observar en forma de porcentaje los participantes que votaron a uno u otro teclado según el parámetro y ranking. A pesar de no poder realizar análisis estadístico debido al bajo tamaño muestral, parece claro que el teclado mediano es el que ha obtenido los mejores resultados en todas las variables: fue encontrado el menos complejo (seleccionado por 3 de 4), estresante (seleccionado por los 4), y cansado (seleccionado por 4 de 4), además del más controlable, preferido (seleccionado por 4 de 4 , en ambas variables) y cómodo (seleccionado por 3 de 4). Por otra parte, el teclado pequeño se 
seleccionó como el más complejo (seleccionado por 3 de 4), más estresante (seleccionado por 4 de 4), el menos cómodo (seleccionado por 3 de 4) y menos controlable (seleccionado por 3 de 4).

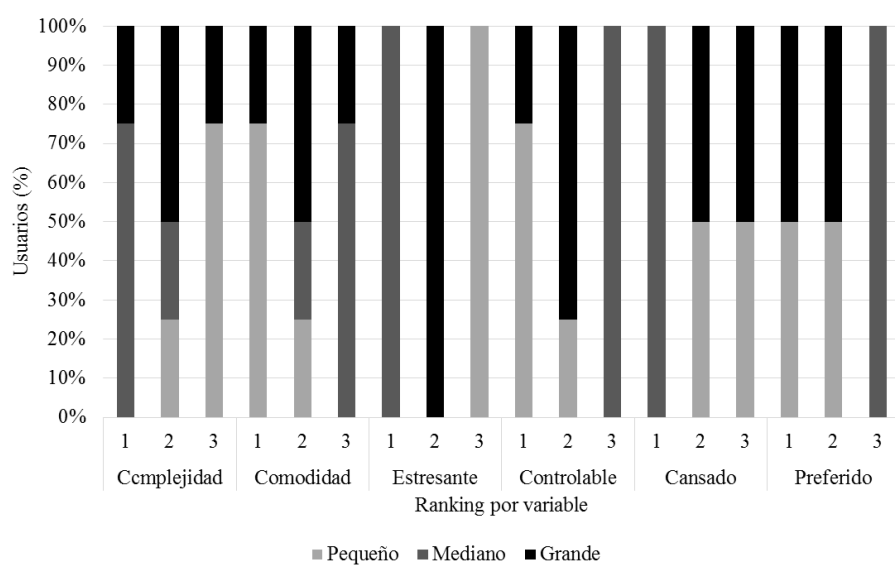

Fig. 4 Resultados obtenidos en el test comparativo. En las barras se presenta el porcentaje de participantes que eligieron un teclado u otro en cada una de los rankings. El Ranking 1 representa 'el menos', el Ranking 2 'el intermedio' y el Ranking 3 'el más'.

La preferencia casi unánime en todas las variables con respecto al teclado mediano, puede ser explicada en parte por la dificultad de los pacientes en fijar su atención en aquellos símbolos alejados del centro (i.e., Q1) con respecto al teclado grande. Por otra parte, la no preferencia del teclado pequeño puede ser explicada por la dificultad en percibir las distintas filas y columnas (i.e., $Q 2$ ).

\section{Conclusiones}

Primero, es importante resaltar que todas las conclusiones no son significativas y que deben ser tomas con cautela. Por otra parte, gracias a los resultados obtenidos en este estudio preliminar, se concluye que el tamaño del teclado sí es relevante para aquellas personas con movilidad reducida. Además, esto también indica que el tamaño de teclado más comúnmente utilizado (i.e., el teclado de tamaño grande) puede no ser el más adecuado a utilizar.

(Ron-Angevin et al., 2019) concluyó que para los sujetos sanos, el mejor tamaño de teclado es el mediano, lo cual lo corroboran las tres dimensiones de la propuesta de usabilidad. Sin embargo, en este caso, cuesta trabajo diferenciar el tamaño óptimo de los resultados de las tres dimensiones. De la parte de eficacia destacan tanto el grande como el mediano, y de la satisfacción se ve la tendencia clara del mediano. Por otra parte, de los tres teclados 
Estudio preliminar de la usabilidad de tres tamaños de teclados basados en Interfaces CerebroComputador por pacientes con ELA

utilizados, solo el teclado pequeño es el que se puede definir como el menos usable viendo el desempeño y puntuaciones obtenidas por los cuatro participantes en las fases de eficacia y satisfacción.

\section{Agradecimientos}

Este trabajo fue parcialmente financiado por el Ministerio Español de Economía y Competitividad a través del proyecto LICOM (DPI2015-67064-R), el Fondo Europeo de Desarrollo Regional (FEDR) y la Universidad de Málaga. Los autores quieren agradecer especialmente a la Fundación Andaluza de ELA y a los participantes por su entusiasmo y cooperación.

\section{Referencias}

ACQUALAGNA, L., TREDER, M. S., \& BLANKERTZ, B. (2013). Chroma Speller: Isotropic visual stimuli for truly gaze-independent spelling. International IEEE/EMBS Conference on Neural Engineering, NER, 1041-1044. https://doi.org/10.1109/NER.2013.6696115

BIRBAUMER, N. (2006). Breaking the silence: Brain-computer interfaces (BCI) for communication and motor control. Psychophysiology, 43(6), 517-532. https://doi.org/10.1111/j.14698986.2006.00456.x

BROOKE, J. (1996). SUS - A quick and dirty usability scale. Usability Evaluation in Industry, 189(194), 4-7. https://doi.org/10.1002/hbm.20701

BRUNNER, P., JOSHI, S., BRISKIN, S., WOLPAW, J. R., BISCHOF, H., \& SCHALK, G. (2010). Does the "P300" speller depend on eye gaze? Journal of Neural Engineering, 7(5). https://doi.org/10.1088/1741-2560/7/5/056013

FARWELL, L. A., \& DONCHIN, E. (1988). Talking off the top of your head: toward a mental prosthesis utilizing event-related brain potentials. Electroencephalography and Clinical Neurophysiology, 70(6), 510-523. https://doi.org/10.1016/0013-4694(88)90149-6

IKEGAMI, S., TAKANO, K., WADA, M., SAEKI, N., \& KANSAKU, K. (2012). Effect of the green/blue flicker matrix for P300-based brain-computer interface: An EEG-fMRI study. Frontiers in Neurology, JUL(July), 1-10. https://doi.org/10.3389/fneur.2012.00113

ISO. (2000). ISO/DIS 9241-9, Ergonomic requirements for office work with visual display terminals. Technical Report, 11, 22. https://doi.org/citeulike-article-id:3503574

KUBlER, A., NEUMANN, N., KAISER, J., KOTCHOUBEY, B., HINTERBERGER, T., \& BIRBAUMER, N. P. (2001). Brain-computer communication: Self-regulation of slow cortical potentials for verbal communication. Archives of Physical Medicine and Rehabilitation, 82(11), 1533-1539. https://doi.org/10.1053/apmr.2001.26621

LI, Q., LIU, S., LI, J., \& BAI, O. (2015). Use of a green familiar faces paradigm improves P300speller brain-computer interface performance. PLOS ONE, 10(6), 1-15. https://doi.org/10.1371/journal.pone.0130325

LI, Y., NAM, C. S., SHADDEN, B. B., \& JOHNSON, S. L. (2011). A p300-based brain-computer 
interface: Effects of interface type and screen size. International Journal of Human-Computer Interaction, 27(1), 52-68. https://doi.org/10.1080/10447318.2011.535753

MCCANE, L. M., SELlERS, E. W., MCFARLAND, D. J., MAK, J. N., CARMACK, C. S., ZEITLIN, D., ... VAUGHAN, T. M. (2014). Brain-computer interface (BCI) evaluation in people with amyotrophic lateral sclerosis. Amyotrophic Lateral Sclerosis and Frontotemporal Degeneration, 15(3-4), 207-215. https://doi.org/10.3109/21678421.2013.865750

NICOLAS-ALONSO, L. F., \& GOMEZ-GIL, J. (2012). Brain computer interfaces, a review. Sensors, 12(2), 1211-1279. https://doi.org/10.3390/s120201211

NIJBOER, F., SELlERS, E. W., MELLINGER, J., JORDAN, M. A., MATUZ, T., FURDEA, A., ... KÜBLER, A. (2008). A P300-based brain-computer interface for people with amyotrophic lateral sclerosis. Clinical Neurophysiology, 119(8), 1909-1916. https://doi.org/10.1016/j.clinph.2008.03.034

PAL, S., MANGAL, N. K., \& KHOSLA, A. (2017). Development of assistive application for patients with communication disability. IEEE International Conference on Innovations in Green Energy and Healthcare Technologies - 2017, IGEHT 2017, 1-4. https://doi.org/10.1109/IGEHT.2017.8094044

PASQUAlOtTO, E., MATUZ, T., FEDERICI, S., RUF, C. A., BARTL, M., OLIVETTI BELARDINELLI, M., ... HALDER, S. (2015). Usability and Workload of Access Technology for People with Severe Motor Impairment: A Comparison of Brain-Computer Interfacing and Eye Tracking. Neurorehabilitation and Neural Repair, 29(10), 950-957. https://doi.org/10.1177/1545968315575611

PATTERSON, J. R., \& GRABOIS, M. (1986). Locked-in syndrome: A review of 139 cases. Stroke, 17(4), 758-764. https://doi.org/10.1161/01.STR.17.4.758

POLETTI, B., CARELli, L., SOlCA, F., LAFRONZA, A., PEDROLI, E., FAINI, A., ... SILANI, V. (2016). Cognitive assessment in Amyotrophic Lateral Sclerosis by means of P300-Brain Computer Interface: a preliminary study. Amyotrophic Lateral Sclerosis and Frontotemporal Degeneration, 17(7-8), 473-481. https://doi.org/10.1080/21678421.2016.1181182

RON-ANGEVIN, R., VARONA-MOYA, S., \& SILVA-SAUER, L. DA. (2015). Initial test of a T9like P300-based speller by an ALS patient. Journal of Neural Engineering, 12(4). https://doi.org/10.1088/1741-2560/12/4/046023

RON-ANGEVIN, R., GARCIA, L., FERNÁNDEZ-RODRIGUEZ, Á, SARACCO, J., ANDRÉ, J.M., \& LESPINET-NAJIB, V. (2019) Impact of speller size in a visual P300-brain-computer interface (BCI) system under two conditions of constraint for eyes movement. Computational Intelligence and Neuroscience, Accepted Manuscript.

RYAN, D. B., COLWELL, K. A., THROCKMORTON, C. S., COLlinS, L. M., CAVES, K., \& SELLERS, E. W. (2018). Evaluating Brain-Computer Interface Performance in an ALS Population: Checkerboard and Color Paradigms. Clinical EEG and Neuroscience, 49(2), 114121. https://doi.org/10.1177/1550059417737443

SALVARIS, M., \& SEPULVEDA, F. (2009). Visual modifications on the P300 speller BCI paradigm. Journal of Neural Engineering, 6(4). https://doi.org/10.1088/1741-2560/6/4/046011

SELLERS, E. W., KRUSIENSKI, D. J., MCFARLAND, D. J., VAUGHAN, T. M., \& WOLPAW, J. R. (2006). A P300 event-related potential brain-computer interface (BCI): The effects of matrix size and inter stimulus interval on performance. Biological Psychology, 73(3), 242-252. https://doi.org/10.1016/j.biopsycho.2006.04.007 


\section{TECNOLOGÍAS PARA LA REHABILTTACIÓN Y LA ASISTENCIA




\title{
Evaluación de la interacción entre atención visual y memoria de trabajo: un estudio basado en técnicas de eye-tracking
}

\author{
Conesa, Natàlia ${ }^{a}$; Balagué, Marta ${ }^{a, b}$ y Dempere-Marco, Laura ${ }^{a^{*}}$ \\ ${ }^{a}$ Grupo de Investigación en Bioinformática y Estadística Médica, Departamento de Ingenierías \& \\ Departamento de Biociencias, Facultad de Ciencias y Tecnología, Universitat de Vic-Universitat \\ Central de Catalunya, \\ b Servicio de Neuropsicología, Hospital Moisès Broggi, Consorci Sanitari de Integral, (*) \\ laura.dempere@uvic.cat
}

\begin{abstract}
Working memory (WM) allows us to hold and manipulate information when it is no longer present to the physical senses. When stimuli are displayed sequentially, recency effects (i.e. stimuli presented later in the sequence are more likely held in WM) have been reported in visual WM. The main hypothesis that guides this study is that visual attention modulates the codification of visual information during the encoding phase of WM. Our results show that the recency effect emerges even when stimuli are shown simultaneously, thus affecting which stimuli enter and are maintained in WM. This effect emerges from the allocation of visual attention to the different items during the stimulation phase of the task, thus illustrating the important role of the interplay between visual attention and WM.
\end{abstract}

Keywords: working memory, attention, aging, eye-tracking, cognitive neuroscience.

\begin{abstract}
Resumen
La memoria de trabajo (MT) nos permite mantener y manipular información cuando ya no está presente a los sentidos. Cuando los estímulos se muestran de forma secuencial se ha descrito la aparición de efectos de recencia (i.e. aquellos estímulos que se muestran en las últimas posiciones de la secuencia son retenidos en MT con mayor probabilidad). La hipótesis principal que subyace este estudio es que la atención visual modula la codificación de información visual durante la fase de estimulación de MT. Nuestros resultados muestran que el efecto de recencia surge incluso cuando los estímulos se muestran de forma simultánea, de modo que determinan qué estímulos entran y son mantenidos en MT. Este efecto es consecuencia de la asignación de atención visual a los diferentes elementos durante la fase de estimulación de la tarea visual, ilustrando asi el importante rol de la interrelación entre atención visual y MT.
\end{abstract}


Palabras clave: memoria de trabajo, atención, envejecimiento, rastreo ocular, neurociencia cognitiva.

\section{Introducción}

La memoria de trabajo (MT) es la función cognitiva que nos permite mantener y manipular información cuando ya no está presente para los sentidos. Esta función cognitiva es básica para el desarrollo de otras funciones cognitivas superiores como la inteligencia, el lenguaje o la memoria (Cowan, 2017). Asimismo, la MT muestra un deterioro con la edad y con la presencia de diversas patologías relacionadas con el envejecimiento, entre ellas las neurodegenerativas. Uno de los mayores retos a los que se enfrenta nuestra sociedad se deriva del incremento en la esperanza de vida de las personas y la necesidad de acompañar la mayor longevidad de una buena calidad de vida. La función cognitiva es clave en este contexto para garantizar la calidad de vida y, por ello, es importante desarrollar técnicas que permitan la identificación de cambios sutiles en el rendimiento cognitivo de las personas. Esto es particularmente crítico en enfermedades como el Alzheimer o el Parkinson, con fases preclínicas muy largas, ya que es precisamente en estas fases en las cuales las terapias pueden ser más efectivas. Este trabajo constituye un avance en la caracterización de perfiles cognitivos, en particular de la MT, al introducir medidas de eyetracking que permiten analizar no sólo el rendimiento de las tareas sino también la dinámica de los procesos que conducen a tales rendimientos (Pallarés et al. 2016, 2017).

\section{Memoria de trabajo y atención visual}

Una característica distintiva de MT es su capacidad limitada. Diversos estudios sugieren que existe un límite absoluto de 3-4 elementos para estímulos visuales (Cowan, 2000, 2017). En la actualidad coexisten varias teorías sobre los mecanismos subyacentes que producen tales límites de capacidad. Los dos modelos principales son los modelos de capacidad fija (o modelos de slots) (Zhang y Luck, 2008) y los modelos de asignación dinámica (o modelos de recursos) (Bays y Husain, 2008). En los modelos de capacidad fija todos los elementos se recuerdan con la misma precisión hasta el límite (3-4 elementos), y se estima que no se almacenará información más allá de este límite. Por el contrario, en los modelos de asignación dinámica, los recursos (finitos) se reparten entre los elementos pero no necesariamente de la misma manera. Un aspecto destacable de este modelo es la predicción de que todos los elementos del conjunto de estimulación reciben ciertos recursos. En este contexto, la atención visual desempeña un rol crucial en la asignación de los recursos que recibe cada estímulo (Melcher y Piazza, 2011). En un estudio computacional Dempere-Marco et al. (Dempere-Marco et al., 2012) retomaron los resultados experimentales de Melcher y Piazza (Melcher y Piazza, 2011) e investigaron los 
mecanismos neuronales responsables de la capacidad limitada de MT y su relación con la relevancia visual, íntimamente relacionada con la atención visual. Tomando estos resultados como punto de partida, en este trabajo se amplía el paradigma experimental utilizado en estos estudios previos para investigar la relación entre la atención visual y la MT.

Para ello, se tiene en cuenta que cuando los estímulos se muestran secuencialmente emergen efectos seriales. En particular, en MT visual se ha observado el efecto de recencia (i.e. es más probable que se recuerden los estímulos presentados en las últimas posiciones de la secuencia). Por el contrario, el efecto de primacía (i.e. es más probable que se recuerden los estímulos presentados al principio de la secuencia) no se ha encontrado en MT visual, aunque sí se ha observado en MT verbal. En (Balagué y Dempere-Marco, 2016) se llevó a cabo un estudio computacional con el objetivo de investigar los mecanismos neuronales de la MT y la emergencia de los efectos seriales. Para estudiar la relación entre la atención visual y MT se ha diseñado un protocolo experimental de coincidencia con una muestra diferida (Delayed Match-to-Sample, DMS), monitorizado mediante técnicas de eye-tracking. Por un lado, se investiga cómo varía la MT con la edad y con el número de objetos presentes en la fase de estimulación. Posteriormente, se analiza hasta qué punto la atención visual modula la codificación de la información durante la fase de estimulación, lo que determina qué estímulos acceden y se mantienen en MT.

\section{Metodología}

\subsection{Descripción del experimento}

\subsubsection{Sujetos experimentales}

Un total de 54 sujetos participaron de forma voluntaria en los experimentos y fueron divididos en 2 cohortes: adultos jóvenes (J) (Edad: 24.10 土2.10 años; 13 hombres, 17 mujeres) y adultos de mediana edad (M) (Edad: 51.63 土5.03 años; 10 hombres, 14 mujeres). En ambas poblaciones, el número de sujetos con niveles de estudios similares fueron balanceados en ambas poblaciones ( $\mathrm{J}$ : 7 estudios medios, 23 estudios superiores; M: 8 estudios medios, 16 estudios superiores). Asimismo, se balanceó el ámbito de estudios (J: 13 artístico-social-humanístico, 17 científico-tecnológico; M: 9 artístico-socialhumanístico, 15 científico-tecnológico). Todos los sujetos participaron en una sesión experimental que se dividía en dos partes: 1) valoración neuropsicológica y 2) tarea DMS. 


\subsubsection{Valoración neuropsicológica}

Tal y como se ha indicado, los sujetos se sometieron a una valoración neuropsicológica exhaustiva con el objetivo de garantizar que su función coginitiva está completamente preservada, es decir, que se trata de individuos sanos desde la perspectiva cognitiva y sin ningún défícit/superávit cognitivo. Se llevaron a cabo un total de 10 pruebas neuropsicológicas en que se valoraron las siguientes funciones (por medio de los tests indicados entre paréntesis): vocabulario, atención y memoria de trabajo (span auditivo (Coalson et al. 2008) y Trial Making Test A (Tamayo, 2012, Peña-Casanova et al. 2009a), habilidades ejecutivas (fluencia semántica (Casals-Coll, 2013; Peña Casanova et al. 2009c ) fluencia verbal (Casals-Coll, 2013; Peña-Casanova et al., 2009c), Stroop (Golden, 1978; Rognoni, 2004; Peña-Casanova, 2009b) y Trial Making Test B (Tamayo, 2012; PeñaCasanova et al., 2009a), memoria (RAVLT (Spreen et al., 2006)), procesamiento de información (clave de números (Coalson et al., 2008)) y habilidades visuoconstructivas (cubos (Coalson et al., 2008)).

\subsubsection{Diseño experimental: tarea DMS monitorizada con eye-tracking.}

Todos los sujetos participaron en una tarea DMS (ver Fig. 1) que consiste en una serie de estímulos cuadrados de colores que se presentaron simultáneamente en matrices de 2, 4 o 6 elementos durante $1000 \mathrm{~ms}$. En este protocolo experimental todos los estímulos se encuentran a la misma distancia del centro de la pantalla (5.5 grados visuales) y, en cada ensayo, hay la misma cantidad de estímulos en cada hemiespacio. Tras $2 \mathrm{~s}$ sin estimulación, se presenta un estímulo de prueba en el centro de la pantalla, durante $0.5 \mathrm{~s}$, para su posterior reconocimiento. El sujeto dispone de $5 \mathrm{~s}$ para contestar por medio de dos botones: verde (objeto presente en conjunto de memorización), o rojo (objeto no presente en conjunto de memorización). Cada experimento consta de 252 ensayos presentados al azar en un diseño equilibrado en el número de estímulos, el color, la ubicación espacial y el estímulo de reconocimiento. Durante el experimento, la mirada de los sujetos fue monitorizada por medio de un eye-tracker (Tobii X120, $f=120 \mathrm{~Hz}$ ). Los estímulos se mostraron en una pantalla de tamaño $33.8 \mathrm{~cm} \times 27 \mathrm{~cm}$ con una resolución de $1280 \times 1080$ y que estaba a una distancia fija de $60 \mathrm{~cm}$ del eye-tracker. Se utilizó un soporte reposabarbillas con el objetivo de minimizar los movimientos de la cabeza durante el experimento, garantizando así un buen mantenimento de la calibración, al tiempo que ofrece una mayor comodidad a los participantes. Los 252 ensayos de dividieron en 3 bloques de 84 ensayos cada uno también balanceados en el número de estímulos, el color, la ubicación espacial y la presencia/ausencia del estímulo de reconocimiento en el conjunto de memorización. 


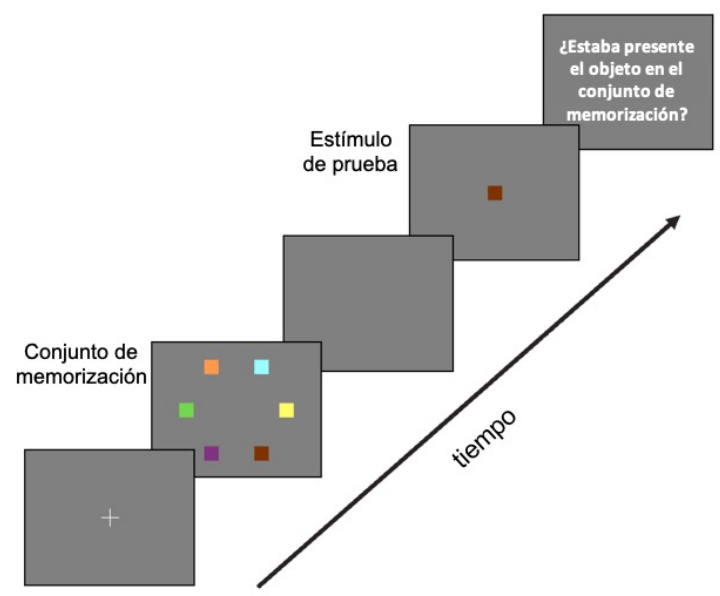

Fig. 1: Ilustración de la tarea DMS. Tras $500 \mathrm{~ms}$ de estabilización de la mirada en una cruz central, se muestra durante $1 \mathrm{~s}$ (fase de codificación/estimulación) el conjunto de memorización (matriz de 2, 4 o 6 de cuadrados de distintos colores ubidados a 5.5 grados visuales del centro). Posteriormente, durante 2 s (fase de memorización sin estímulos) se muestra una pantalla sin estímulos. Al acabar, se muestra el estímulo de prueba durante $500 \mathrm{~ms}$ (fase de prueba), seguido de la pregunta: “Estaba presentes el objeto en el conjunto de memorización?”. El suejo experimental dispone de 5 s para contestar. Todo el ensayo es monitorizado mediante un eye-tracker Tobii X120.

Los colores de los estímulos fueron definidos en un estudio previo de modo que fuesen claramente distinguibles entre ellos al tiempo que se minimizasen los efectos de prominencia visual (visual saliency) ya que pueden sesgar los resultados comportamentales. La descripción cuantitativa de los colores en sus componentes RGB se describen en la Tabla 1.

Tabla 1. Descripción cuantitativa de los colores de los estímulos en el espacio RGB.

\begin{tabular}{cccc}
\hline Color & R & G & B \\
\hline Naranja & 1 & 0.6 & 0.3 \\
\hline Verde & 0.45 & 0.85 & 0.3 \\
\hline Lila & 0.5 & 0.2 & 0.5 \\
\hline Amarillo & 1 & 1 & 0.4 \\
\hline Cian & 0.6 & 1 & 1 \\
\hline Marrón & 0.5 & 0.2 & 0 \\
\hline Rosa & 1 & 0.6 & 1 \\
\hline
\end{tabular}




\subsection{Análisis de datos}

\subsubsection{Rendimiento en la tarea}

De la tarea DMS, independientemente de los datos de eye-tracking, se han evaluado una serie de parámetros con el objetivo de investigar si existe algún sesgo comportamental en función del color de los estímulos visuales (errores en función del color), si varía el rendimiento en función del número de estímulos (errores en función del número de estímulos), la tipología de respuestas correctas e incorrectas (matriz de confusión), si hay aprendizaje a lo largo del experimento (evolución de la proporción de errores a lo largo del experimento) así como la dinámica particular de cada tipo de respuesta (tiempo medio de respuesta en función del tipo de respuesta). En particular, se han llevado a cabo los siguientes estudios:

- Rendimiento global. Análisis de la frecuencia de aciertos y errores de ambas poblaciones experimentales.

- Errores en función del número de estímulos. Errores que cometen los sujetos experimentales según el número de estímulos que aparecen en la fase de memorización.

- Matriz de confusión. Se distinguen cuatro posible respuestas (ver Tabla 2): positivos verdaderos (PV; el estímulo test está presente en el conjunto de memorización y el sujeto experimental responde correctamente que está presente), falsos positivos (FP; el estímulo test no está presente en el conjunto de memorización y el sujeto experimental responde erróneamente que está presente), negativos verdaderos (NV; el estímulo test no está presente en el conjunto de memorización y el sujeto experimental responde correctamente que no está presente) y falsos negativos (FN; el estímulo test está presente en el conjunto de memorización y el sujeto experimental responde que no está presente).

Tabla 2. Matriz de confusión

\begin{tabular}{|c|c|c|c|}
\cline { 3 - 4 } \multicolumn{2}{c|}{} & \multicolumn{2}{c|}{ Respuesta } \\
\cline { 3 - 4 } \multicolumn{2}{c|}{} & Correcta & Incorrecta \\
\hline \multirow{2}{*}{ Estímulo } & Presente & VP & FN \\
\cline { 2 - 4 } & Ausente & VN & FP \\
\hline
\end{tabular}

- Tiempo medio de respuesta en función del tipo de respuesta. Tiempo medio que tarda el sujeto experimental en presionar el botón en función de la categorización de la respuesta en la matriz de confusión. 


\subsubsection{Análisis de datos eye-tracking: efecto de recencia.}

Con el objetivo de analizar la interrelación entre la atención visual y la MT, se ha llevado a cabo un análisis específico de los datos de eye-tracking para evaluar si aquellos estímulos que se miran en las últimas posiciones de una secuencia tienen mayor probabilidad de ser recordados, es decir, si emerge un efecto de recencia. Para ello, sólo se han considerado los ensayos en que se muestran 6 colores y en los que el estímulo test se encuentra presente en el conjunto de memorización. Por tanto, en este estudio se trabaja sobre una base de 42 ensayos para cada sujeto.

Cabe destacar que el experimento se ha realizado bajo un paradigma experimental de búsqueda libre, de modo que los sujetos no reciben ninguna instrucción sobre cómo deben mirar el conjunto de memorización. Esto implica que aparezca una variedad de estrategias de visualización de los estímulos. La Fig. 2 muestra tres tipologías de exploración visual del conjunto de memorización diferentes: visualización periférica, visualización exhaustiva, visualización de mínima búsqueda.

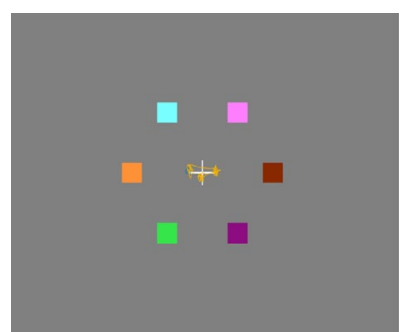

(a)

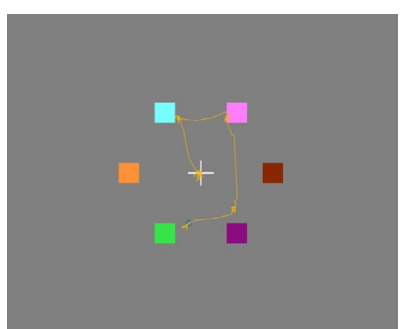

(b)

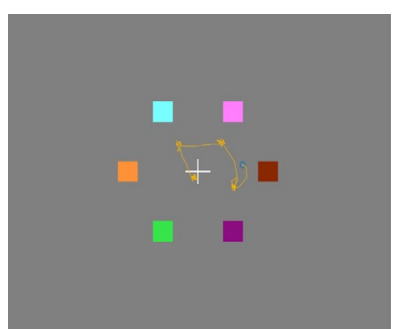

(c)

Fig. 2: Ilustración de tres estrategias de visualización diferentes durante la fase de memorización en la tarea DMS. La línea amarilla representa la trayectoria de exploración de la imagen que han seguido los sujetos en los tres ensayos considerados. (a) Visualización periférica: el sujeto mantiene la mirada en la zona central y explora los colores por medio de visión periférica, (b) Visualización exhaustiva: el sujeto observa de forma abierta y deliberada diversos estímulos durante la fase de memorización, y (c) Visualización de mínima búsqueda: el sujeto realiza una exploración abierta del conjunto de memorización pero no no fija la mirada en los estímulos.

En los análisis realizados en esta sección, sólo se han considerado las exploraciones de tipo exhaustivo. De los datos de eye-tracking se ha obtenido la secuencia de visualización de los diferentes estímulos. Un estímulo se considera procesado si el sujeto pasa un mínimo de $100 \mathrm{~ms}$ consecutivos en la zona de interés que, en nuestro estudio, se define como una región de 3 × 3 grados visuales centrados en cada estímulo. 


\section{Resultados}

\subsection{Rendimiento en la tarea DMS}

Tal y como se puede observar en Fig. 3(a), globalmente, los adultos jóvenes obtuvieron más respuestas correctas que los adultos de mediana edad $(t=3.108, p<0.005)$. Si se distingue entre el número de estímulos del conjunto de memorización, Fig. 3(b), se produjeron diferencias significativas entre las dos cohortes para 4 estímulos $(t=-3.031, p<0.005)$ y 6 estímulos $(t=-2.892, p=0.005)$ pero no para 2 estímulos. $(t=-0.675, p=0.502)$.

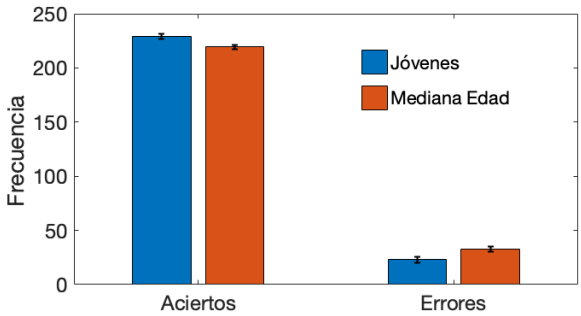

(a)

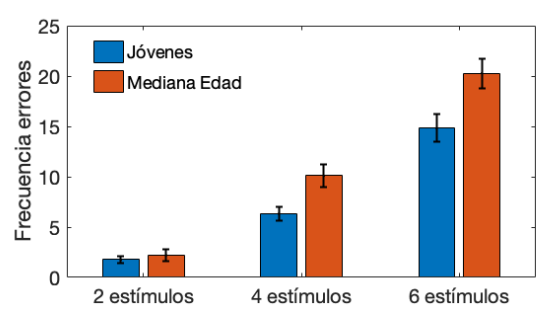

(b)

Fig. 3 (a) Frecuencia de errores y aciertos en la tarea DMS para las poblaciones $J$ y M, (b) frecuecnia de errores en la tarea para las poblaciones $J$ y M y variación en función del número de estímulos presentes en el conjunto de estimulación.

Tabla 3. Comparación del rendimiento de las poblaciones $\mathrm{J}$ y $\mathrm{M}$ en la tarea DMS.

\begin{tabular}{|ccc|}
\hline & $\boldsymbol{t}$-test & $\boldsymbol{p}$ \\
Aciertos & 3.108 & 0.003 \\
Errores & -3.108 & 0.003 \\
\hline
\end{tabular}

Tabla 4. Comparación del comportamiento de las poblaciones $\mathrm{J}$ y $\mathrm{M}$ en función de los errores cometidos para distintos tamaños del conjunto de memorización.

\begin{tabular}{|ccc|}
\hline \# Estímulos & $\boldsymbol{t}$-test & $\boldsymbol{p}$ \\
2 & -0.675 & 0.502 \\
4 & -3.031 & 0.004 \\
6 & -2.892 & 0.005 \\
\hline
\end{tabular}

Cuando se analizan por separado los tres bloques se observa que hay diferencias significativas en el rendimiento de ambas poblaciones en el primer bloque en que la población $\mathrm{J}$ muestra un rendimiento superior $(t=-3.983, p<0.001$, ver Tabla 5). Asimismo, si se atiende al rendimiento a lo largo de la tarea, se desprende que existen diferencias significativas entre el rendimiento en la tarea a lo largo de los bloques, tanto para la población de jóvenes adultos ( $\mathrm{J}$ ) (ANOVA medidas repetidas, $F=5.389, p=0.010$ ), como en la población de adultos de mediana edad (M) (ANOVA medidas repetidas $F=$ $22.914, p<0.001)$. Por tanto, se puede concluir que se produce un aprendizaje a lo largo de la tarea. 


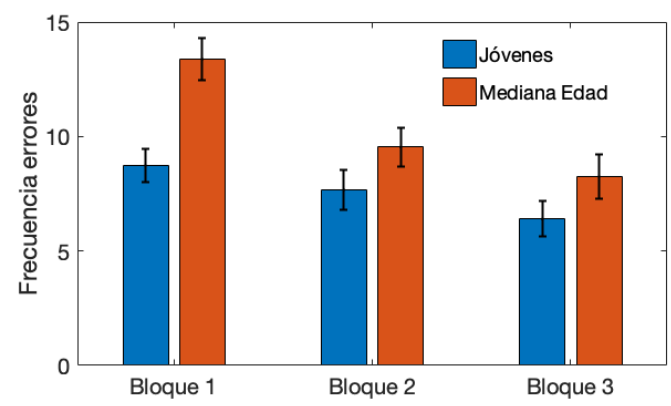

Fig. 4 Frecuencia de errores en la tarea DMS para las poblaciones $J$ y $M$ en función del bloque experimental. El bloque 1 se realiza en primer lugar, seguido de los bloques 2 y 3, consecutivamente.

Tabla 5. Comparación del rendimiento de las poblaciones $J$ y $\mathbf{M}$ en función del bloque experimental.

\begin{tabular}{|ccl|}
\hline & $\boldsymbol{t}$-test & \multicolumn{1}{c|}{$\boldsymbol{p}$} \\
1 & -3.983 & $<0.001$ \\
2 & -1.488 & 0.143 \\
3 & -1.473 & 0.147 \\
\hline
\end{tabular}

Otro modo de analizar y de comparar el rendimiento de las dos poblaciones es atendiendo a la tipología de los aciertos y de los errores (ver Tablas 6 y 7). Aunque el número de aciertos de la población joven aumenta respecto al de la población de mediana edad, esta diferencia no alcanza valores estadísticamente significativos ni en el caso de PV ni en el de FN. Sin embargo, sí que se aprecian diferencias significativas en el caso de los $\mathrm{FP}(t=-3.360, p=$ $0.001)$ y NV ( $t=-3.360, p=0.001)$ lo cual puede interpretarse como un empobrecimiento en la capacidad de codificar la información que llega a los sentidos con la edad.

Tabla 6. Matriz confusión promedio obtenida a partir de datos comportamentales de la población joven.

\begin{tabular}{|l|c|c|c|}
\cline { 3 - 4 } \multicolumn{2}{c|}{} & \multicolumn{2}{c|}{ Respuesta } \\
\cline { 3 - 4 } \multicolumn{2}{c|}{} & Correcta & Incorrecta \\
\hline \multirow{2}{*}{ Estímulo } & Presente & 117.17 & 7.97 \\
\cline { 2 - 4 } & Ausente & 118.03 & 14.83 \\
\hline
\end{tabular}

Tabla 7. Matriz confusión confusión promedio obtenida a partir de datos comportamentales de la población mediana edad.

\begin{tabular}{|c|c|c|c|}
\cline { 3 - 4 } \multicolumn{2}{c|}{} & \multicolumn{2}{c|}{ Respuesta } \\
\cline { 3 - 4 } \multicolumn{2}{c|}{} & Correcta & Incorrecta \\
\hline \multirow{2}{*}{ Estímulo } & Presente & 109.67 & 19.42 \\
\cline { 2 - 4 } & Ausente & 111.17 & 16.33 \\
\hline
\end{tabular}

Asimismo, si nos fijamos en los tiempos que se tarda en emitir una respuesta, no se observan diferencias significativas entre ambas poblaciones para ninguno de los tipos de situaciones identificadas en la matriz de confusión (i.e. $p>0.05$ en todos los casos). Sin embargo, llama la atención que las respuestas incorrectas, y especialmente, los FN tienen 
asociados tiempos de reacción mayores, sugiriendo de este modo que la información visual ha sido codificada en algún nivel (ver Tablas 8 y 9).

Tabla 8. Matriz confusión promedio obtenida a partir de datos comportamentales de la población joven.

\begin{tabular}{|c|c|c|c|}
\cline { 3 - 4 } \multicolumn{2}{c|}{} & \multicolumn{2}{c|}{ Respuesta } \\
\cline { 3 - 4 } \multicolumn{2}{c|}{} & Correcta & Incorrecta \\
\hline \multirow{2}{*}{ Estímulo } & Presente & $0.46 \mathrm{~s}$ & $1.18 \mathrm{~s}$ \\
\cline { 2 - 4 } & Ausente & $0.51 \mathrm{~s}$ & $0.89 \mathrm{~s}$ \\
\hline
\end{tabular}

Tabla 9. Matriz confusión confusión promedio obtenida a partir de datos comportamentales de la población mediana edad.

\begin{tabular}{|c|c|c|c|}
\cline { 3 - 4 } \multicolumn{2}{c|}{} & \multicolumn{2}{c|}{ Respuesta } \\
\cline { 3 - 4 } \multicolumn{2}{c|}{ Estímulo } & Correcta & Incorrecta \\
\cline { 2 - 4 } & Presente & $0.50 \mathrm{~s}$ & $1.06 \mathrm{~s}$ \\
\hline
\end{tabular}

\subsection{Efecto recencia}

En relación a los efectos seriales, el estudio se centró en aquellos ensayos en que se mostraron 6 estímulos. De éstos, en el 26\% de los 84 ensayos/persona tuvieron lugar búsquedas exhaustivas (las secuencias de fijaciones están totalmente contenidas en ROIs de $3 \times 3$ grados visuales centrados en los estímulos). Con el objetivo de analizar efectos seriales se han considerado aquellas secuencias que tienen una longitud $N>2$. En nuestro estudio, $N_{\max }=4$. Por tanto, se han analizado (de forma independiente para cada tamaño) las secuencias que contienen 3 y 4 fijaciones. Finalmente, se han agregado los datos de las secuencias de longitud 3 y 4 , alineando las secuencias respecto al último estímulo observado. Esto permite observar si existe un efecto serial en las posiciones $F, F-1$ y $F-$ 2, siendo $F$ la última posición de la secuencia, $F$-1 la penúltima y $F-2$ la antepenúltima.

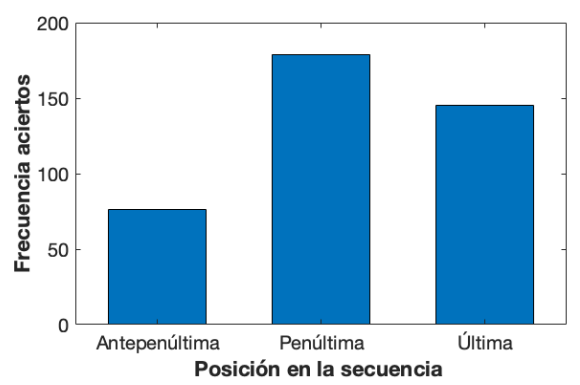

Fig. 5: Efectos seriales en el rendimiento de la tarea. Se muestra la frecuencia con que los sujetos experimentales han dado una respuesta correcta en función de la posición en la secuencia en que han observado el estímulo test.

Tal y como se puede observar en la Fig. 5, los 2 últimos estímulos que recibieron atención abierta durante la búsqueda fueron reconocidos significativamente mejor que los estímulos visitados con anterioridad durante la fase de codificación (desde la posición final, $1^{\mathrm{o}}$ vs $2^{\mathrm{o}}$ $\chi^{2}=2.382 p=0.140 ; 2^{\circ}$ vs $3^{\circ} \chi^{2}=28.248, p<0.001$ ) sugieriendo de este modo la aparición de 
un efecto de recencia y poniendo de relieve la importancia de la interacción entre el despliegue de atención visual y la MT.

\section{Conclusiones}

En este trabajo se ha investigado la memoria de trabajo en el marco de una tarea DMS y su deterioro con la edad. Para ello, se han reclutado dos grupos de sujetos de diferente edad (jóvenes adultos, $\mathrm{J}$, y adultos de mediana edad, M) y se ha diseñado un protocolo experimental que permite combinar la tarea DMS con medidas de eye-tracking. Los resultados obtenidos están de acuerdo con las previsiones de estudios computacionales anteriores llevados a cabo en nuestro grupo (Dempere-Marco et al, 2012; Rolls et al., 2013, Balagué y Dempere-Marco, 2016). En particular, las predicciones de los modelos computacionales indican que la memoria de trabajo yace en un continuo entre las predicciones de los modelos slot (Zhang y Luck, 2008) y los modelos de recursos compartidos (Bays y Husain, 2008). En este contexto, la atención visual desempeña un papel crucial tal y como se investigó en términos de la relevancia visual y su rol en los procesos atencionales de carácter bottom-up en (Piazza y Melcher, 2011) y (DempereMarco et al., 2012).

En primer lugar, nuestros resultados aportan evidencia experimental sobre la disminución de la capacidad de MT con la edad y con el tamaño del conjunto de memorización. Además, en este estudio la introducción de técnicas de eye-tracking en el protocolo experimental permite analizar cómo se despliega la atención visual abierta durante el período de codificación de los estímulos. Los resultados obtenidos respaldan la hipótesis de que la atención visual modula la codificación de los elementos en MT. En particular, el efecto de recencia surge como consecuencia de la asignación de atención visual a los diferentes elementos durante la fase de estimulación de la tarea DMS, lo que ilustra el papel tan importante que tiene la interacción entre la atención visual y MT.

\section{Agradecimientos}

Las autoras agradecen a Laura Portell su colaboración en el proyecto de investigación en el marco del cual contribuyó en el diseño del protocolo experimental de la tarea DMS. Esta investigación ha sido posible gracias a la financión recibida por parte Ministerio de Ciencia e Innovación a través del proyecto TIN2013-40630-R.

\section{Referencias}

BALAGUE, M., DEMPERE-MARCO, L. (2016) Multi-item Working Memory Capacity: What Is the Role of the Stimulation Protocol? In Lecture Notes in Computer Science Vol. 9886: 264-271. 
Evaluación de la interacción entre atención visual y memoria de trabajo: un estudio basado en técnicas de eye-tracking

BAYS, P., HUSAIN, M., (2008) "Dynamic shifts of limited working memory resources in human vision" en Science Vol. 321, pp. 851-854.

CASALS-COLL, M. (2013) "Estudios normativos españoles en población adulta joven (proyecto NEURONORMA jóvenes): normas para los test de fluencia verbal” en Neurología, Vol. 28 (1), pp. 33-40.

COALSON D. L. RAIFORD S. E. WECHSLER, D. (2008) "WAIS-IV: Wechsler adult intelligence scale", San Antonio TX Pearson.

COWAN, N. (2000) "The magical number 4 in short-term memory: A reconsideration of mental storage capacity" in Behavioural Brain Science, Vol. 24, pp. 87-116.

COWAN, N. (2017) "The many faces of working memory and short-term storage" in Psychonomic Bulletin Review, Vol. 24, pp. 1158-1170.

DEMPERE-MARCO, L., HU, X-P., YANG, G.Z., (2011) "A novel framework for the analysis of eye movements during visual search for knowledge gathering" en Cognitive Computation, Vol. (3) 1, pp. 206-222

DEMPERE-MARCO, L., MELCHER, D. P., DECO, G. (2012) "Effective visual working memory capacity: an emergent effect from the neural dynamics in an attractor network" en PLoS One, Vol. 7 (8): e42719. https://doi.org/10.1371/journal.pone.0042719 [Consulta: 15 de mayo de 2019]

GOLDEN, CJ. (1978) "Stroop Color and Word Test: a manual for clinical and experi- mental uses", Wood Dale: Stoeling Company.

MELCHER, D., PIAZZA, M. (2011) "The role of attentional priority and saliency in determining capacity limits in enumeration and visual working memory" en PLoS One Vol. 6: 1-11 [Consulta: 15 de mayo de 2019]

PALLARES, V., HERNANDEZ, M., DEMPERE-MARCO, L. (2017) "Eye-Tracking Data in Visual Search Tasks: A Hallmark of Cognitive Function" en Converging Clinical and Engineering Research on Neurorehabilitation II, 873-877.

PALLARES, V., RAMI, L., DEMPERE-MARCO, L. (2016), “A Novel Method for Disentangling Strategies from Visual Search“" en BIOSIGNALS, 271-276

PEÑA-CASANOVA, J., QUIÑONES-UBEDA, S., QUINTANA-APARICIO, M., AGUILAR, M., BADENES, D., MOLINUEVO, JL., TORNER, L., ROBLES, A., BARQUERO, MS., VILLANUEVA, C., ANTUNEZ, C., MARTINEZ-PARRA, C., FRANK-GARCIA, A., SANZ, A., FERNANDEZ, M., ALFONSO, V., SOL, JM., BLESA, R. (2009a) "Spanish Multicenter Normative Studies (NEURONORMA Project): norms for verbal span, visuospatial span, letter and number sequencing, trail making test, and symbol digit modalities test", en Archives of Clinical Neuropsychology, Vol. 24 (4), pp. 321-341.

PEÑA-CASANOVA, J., QUIÑONES-UBEDA, S., QUINTANA-APARICIO, M., AGUILAR, M., BADENES, D., MOLINUEVO, JL., TORNER, L., ROBLES, A., BARQUERO, MS., VILLANUEVA, C., ANTUNEZ, C., MARTINEZ-PARRA, C., FRANK-GARCIA, A., SANZ, A., FERNANDEZ, M., ALFONSO, V., SOL, JM., BLESA, R. (2009b) "Spanish Multicenter Normative Studies (NEURONORMA Project): norms for the Stroop color-word interference test and the Tower 
of London-Drexel", en Archives of Clinical Neuropsychology, Vol. 24 (4), pp. 413-429.

PEÑA-CASANOVA， J., QUIÑONES-ÚBEDA， S., GRAMUNT-FOMBUENA， N., QUINTANA-APARICIO, M., AGUILAR, M., BADENES, D., BARQUERO, M. S. (2009C). "Spanish Multicenter Normative Studies (NEURONORMA Project): norms for verbal fluency tests" en Archives of Clinical Neuropsychology, Vol. 24 (4), pp. 395-411.

ROGNONI, T. (2004) "Estudios normativos españooles en población adulta joven (proyecto NEURONORMA jóvenes) normas para las pruebas Stroop Color-Word Interference Test y Tower of London-Drexel University” en Psychological Sciences, Vol. 15:106-111.

ROLLS, E.T., DEMPERE-MARCO, L., DECO, G. (2013) "Holding multiple items in short term memory: a neural mechanism" en PLoS One, Vol. 8 (4): e61078. https://doi.org/10.1371/journal.pone.0061078 [Consulta: 15 de mayo de 2019]

SPREEN, O., STRAUSS, E., SHERMAN, EMS. (2006) “A compendium of neuropsychological test. Administration, norms, and commentary” New York: Oxford University Press.

TAMAYO, F. (2012) "Estudios normativos españoles en población adulta joven (Proyecto NEURONORMA jóvenes): normas para las pruebas span verbal, span vi- suoespacial, Letter-Number Sequencing, Trail Making Test y Symbol Digit Modalities Test” en Neuroloía, Vol. 27 (6), pp. 319 329.

YANG, GZ., DEMPERE-MARCO, L., HU, XP., ROWE, A. (2002) "Visual search: psychophysical models and practical applications" en Image and Vision Computing, Vol. 20 (4), pp. 291-305

ZHANG, W., LUCK, S. (2008) "Discrete fixed-resolution representations in visual working memory" en Nature, Vol. 453, pp. 233-235. 


\title{
Consecuencias de la neuromodulación endógena en el fenómeno de la desincronización de los ritmos sensorimotores en la enfermedad de Parkinson
}

\author{
del Castillo-Sobrino, M. Dolores; Serrano-Moreno, J. Ignacio y Rocon-de Lima, \\ Eduardo \\ Neural and Cognitive Engineering Group ( $\mathrm{gNeC}$ ) del Centro de Automática y Robótica (CAR) CSIC- \\ UPM, Ctra. Campo Real, km. 0,200, 28500 Arganda del Rey, Madrid, Spain, emails: \\ jignacio.serrano@,csic.es, e.rocon@csic.es, md.delcastillo@,csic.es
}

\begin{abstract}
This paper shows the results of a study of the desynchronization of the sensorimotor rhythms in Parkinson's disease (PD) after performing eight sessions of an endogenous neuromodulation intervention. Electrophysiological cortical activity is recorded while patients execute a wrist extension motor task before and after neuromodulation sessions. The goal of this study is included within a major objective aiming to compare the therapeutic efficacy of different neuromodulation strategies in PD patients.
\end{abstract}

Keywords: Parkinson's disease, sensorymotor rhytnms, neuromodulation.

\section{Resumen}

En este artículo se presentan los resultados de un estudio sobre los cambios que se producen en los ritmos sensorimotores de pacientes de la Enfermedad de Parkinson (EP) tras realizar ocho sesiones de neuromodulación endógena. La actividad electroencefalográfica analizada se registra mientras los pacientes realizan una tarea motora de extensión de muñeca antes y después de las sesiones de neuromodulación. El objetivo de este estudio se engloba dentro de un objetivo más amplio que busca comparar la eficacia terapéutica de diferentes técnicas de neuromodulación en EP.

Palabras clave: Enfermedad de Parkinson, ritmos sensorimotores, neuromodulación. 


\section{Introducción}

El movimiento voluntario es una seña de identidad del comportamiento humano. La planificación y ejecución de un movimiento es el resultado del procesamiento paralelo y distribuido de información entre diferentes áreas cerebrales corticales y subcorticales. La naturaleza oscilatoria, que gobierna la actividad cerebral, puede detectarse mediante señales periódicas, caracterizadas por un rango de frecuencia y una distribución topográfica, que a través de electroencefalografía (EEG). El estudio de estas oscilaciones en la corteza premotora, el área motora suplementaria, la corteza motora primaria y la corteza somatosensorial puede proporcionar conocimiento sobre aspectos inherentes a los procesos motores (Mostile, 2015).

La preparación y realización de un movimiento supone un evento que bloquea la actividad electroencefalográfica espontánea en determinadas bandas de frecuencia como consecuencia de cambios en algunos de los parámetros que controlan las oscilaciones de las redes neuronales implicadas en el mismo. Estos cambios relacionados con el movimiento se localizan en la corteza sensorimotora y se manifiestan mediante la desincronización (ERD, del inglés event-related-desynchronization) de la señal EEG, principalmente en las bandas de frecuencia alpha y beta. El fenómeno neurofisiológico de la desincronización conlleva una disminución de la potencia de la señal en esas bandas respecto de la potencia de la señal en un periodo previo al movimiento (Pfurtscheller, 1999). La desincronización del ritmo alpha es más destacado en la corteza contralateral sensorimotora, durante la preparación del movimiento, y se propaga simétricamente a ambos hemisferios cuando se inicia el movimiento. Las oscilaciones en la banda beta en esta área se bloquean durante la ejecución de un movimiento y con la estimulación somatosensorial (Neuper, 2006). En general, la desincronización de los ritmos alpha y beta refleja cambios en la actividad de las interacciones locales entre las neuronas e interneuronas de la corteza sensorimotora que afectan a las componentes en frecuencia de la señal electroencefalográfica espontánea.

Existe una correlación entre los parámetros y la morfología del fenómeno de la desincronización y la disfuncionalidad motora presente en trastornos del sistema nervioso central como el accidente cerebro vascular (ACV) y la enfermedad de Parkinson (EP) (Pfurtscheller, 1999) y su análisis puede conducir a mejorar el diagnóstico y la evolución de este tipo de enfermedades.

La EP se caracteriza por la progresiva degeneración de las neuronas dopaminérgicas de la sustancia negra que se acompaña de síntomas motores característicos como la lentitud de movimiento, la rigidez y la inestabilidad postural (Fahn, 2011). La medicación dopaminérgica produce normalmente una mejoría de los síntomas motores primarios pero 
algunos de ellos, como la dificultad en la marcha o la inestabilidad postural, apenas se modifica e incluso otros, como la disquinesia o la impulsividad, empeoran (Fahn, 2006). A pesar de ello, el tratamiento farmacológico es el mejor para esta enfermedad.

En la EP, los estudios realizados sobre el fenómeno de la desincronización de los ritmos sensorimotores apuntan a que, en la preparación del movimiento, éste está menos lateralizado sobre el área sensorimotora contralateral y se inicia con posterioridad a los controles sanos (Pfurtscheller, 1999). Por otro lado, muchos estudios neurofisiológicos llevados a cabo en este tipo de pacientes han descrito la presencia de una excesiva sincronización de los ritmos beta en el circuito formado por los ganglios basales, el tálamo y la corteza sensorimotora durante el reposo (Hutchison, 2014), (Sharott, 2014), que está asociada a la duración de la enfermedad y a la lentitud de los movimientos (Gulberti, 2015). Esta activación patológicamente alta, que muestra una escasa modulación durante la realización de un movimiento o tras la práctica repetitiva de tareas motoras (Moisello, 2015), puede normalizarse con el tratamiento dopaminérgico y la estimulación cerebral profunda así como con protocolos de regulación, como la neuromodulación endógena, de los patrones oscilatorios específicos motores (Esmail, 2014).

En (Heida, 2014) analizan mediante EEG la escasa desincronización del ritmo alpha en la corteza motora en pacientes con EP durante la observación de un movimiento en contraste con la desincronización bilateral experimentada por sujetos sanos. En pacientes con EP sin medicación se produce una exagerada desincronización del ritmo beta en la corteza motora suplementaria durante la realización de un movimiento, que se normaliza con el tratamiento dopaminérgico (Chung, 2018). En (Kondylis, 2016) se estudian las diferencias en la desincronización de los ritmos alpha y beta que produce una tarea motora entre pacientes con EP y con Temblor esencial a través de electrocorticografía.

Apenas existen en la literatura estudios sistemáticos en pacientes con EP sobre los parámetros de los ritmos sensorimotores mientras realizan una tarea motora, basados en el análisis de la actividad EEG, antes y después de la aplicación de intervenciones de neuromodulación.

En (Esmail, 2014) se realiza una revisión de varios trabajos de neuromodulación endógena en pacientes con EP y, en la mayoría de ellos, la evaluación de sus efectos se circunscribe al ámbito cinemático y motor (equilibrio, disquinesias, tiempos de reacción). El trabajo de (Fumuro, 2013) pone el foco en los potenciales evocados y en los potenciales corticales lentos en la corteza motora tras un procedimiento de neurorregulación de los potenciales lentos en el área motora central. Los autores de (Subramanian, 2011) evalúan la activación de áreas corticales premotoras y subcorticales, mediante resonancia magnética funcional, en una tarea de imaginación de movimiento. El trabajo de (Erickson, 2012) expone cómo la 
modulación exógena de los ritmos beta empleando estímulos auditivos produce una mejoría de la respuesta motora en EP.

En este trabajo se lleva a cabo el análisis de un conjunto de variables que caracterizan estructural y temporalmente el fenómeno neurofisiológico de la desincronización de los ritmos alpha y beta en la corteza sensorimotora en pacientes con EP. El estudio calcula estas variables antes y después de que los pacientes con EP se hayan sometido a una intervención de neuromodulación endógena.

\section{Neuromodulación de los ritmos sensorimotores}

El estudio aquí presentado se enmarca dentro de un proyecto de investigación cuyo objetivo general es el de evaluar y comparar la eficacia terapéutica de dos tipos de intervenciones de neuromodulación, una exógena, basada en la aplicación de estimulación magnética transcraneal repetitiva (rTMS) y, otra endógena, basada en mecanismos de autorregulación de la señal EEG a través de un entorno de realidad virtual. En ambos casos, se persigue modificar el sistema nervioso central, en base a la plasticidad neuronal, para producir mejoras de los síntomas motores mediante potenciación o inhibición de la actividad neuronal de la corteza sensorimotora.

Para ello, el proyecto ha contado con 40 pacientes con EP y 25 sujetos de control sanos. Los pacientes con EP se han dividido en un grupo de control y tres grupos experimentales. Cada grupo experimental ha llevado a cabo un tipo distinto de intervención de neuromodulación (neuromodulación endógena, neuromodulación exógena, una combinación de ambas). Las intervenciones de neuromodulación han tenido lugar en 8 sesiones a lo largo de dos semanas.

Antes y después de cada intervención de neuromodulación, los grupos experimentales han realizado una batería de tests clínicos motores, neurológicos, neuropsicológicos y de estabilidad. Además, se ha registrado su actividad electroencefalográfica durante la realización de dos tareas motoras (extensión de muñeca y unión repetitiva de los dedos índice y pulgar). Los grupos de control con EP y los sanos han sido valorados en una etapa inicial. Para el grupo de control con EP, también se ha llevado a cabo su valoración en una etapa posterior, dos semanas después.

En este artículo se presenta el análisis de la actividad oscilatoria presente en la señal EEG durante la realización de una de las tareas motoras en el grupo experimental sometido a la neuromodulación endógena y de las diferencias encontradas con el grupo de control sano. 


\section{Métodos y materiales}

\subsection{Población de estudio}

En este estudio han participado 11 pacientes con EP (4 mujeres, 7 hombres; diestros; edad media: $62.18 \pm 8.18$ años; tiempo desde el diagnóstico: $5.91 \pm 3.75$ años; Escala Unificada para la Enfermedad de Parkinson UPDRS: 15.55 \pm 7.29 , 5-32; dosis equivalente a levodopa (mg): 443 $\pm 218,100-710)$ y 25 sujetos sanos pareados por edad (6 mujeres, 19 hombres, edad media: 69.64 \pm 9.54 años). Ningún paciente con EP presentaba déficits cognitivos. Todos los pacientes realizaron este protocolo con el efecto de la medicación dopaminérgica, conocido como estado ON.

\subsection{Protocolo experimental y medición EEG}

Todos los sujetos permanecieron sentados durante la realización de una tarea motora que consistía en una tanda de 15 ensayos de extensión de muñeca, primero con la mano derecha y después con la mano izquierda, siguiendo un protocolo guiado a través de la pantalla de un ordenador portátil. En la Figura 1 se muestra el protocolo.

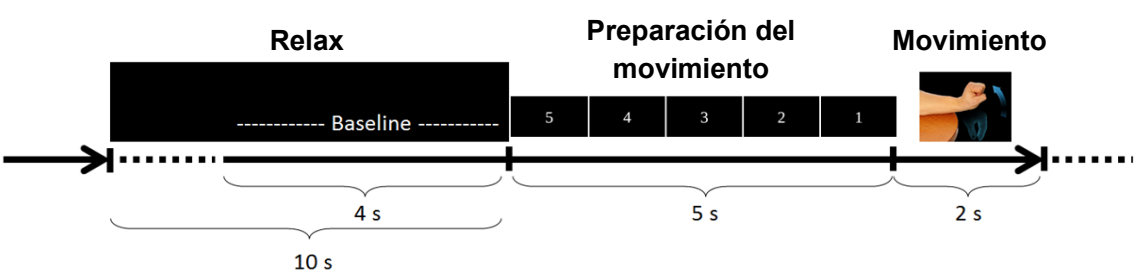

Fig. 1 Protocolo experimental guiado de la tarea motora de extensión de muñeca.

Durante esta tarea, se registraron las señales EEG de nueve canales situados en las áreas premotora, motora y somatosensorial (FC3, FCz, FC4, C3, Cz, C4, CP3, CPz y CP4 de acuerdo al sistema internacional 10-20) utilizando electrodos activos $\mathrm{Ag} / \mathrm{AgCl}$ (Acticap, Brain Products $\mathrm{GmbH}$, Germany). Los electrodos de tierra y referencia se ubicaron en $\mathrm{AFz}$ y $\mathrm{FCz}$, respectivamente. Las señales se amplificaron (BrainVision actiCHamp, Brain Products $\mathrm{GmbH}$, Germany) y se muestrearon a $250 \mathrm{~Hz}$. La adquisición de la señal EEG se llevó a cabo con el software NeuroRT Studio (Mensia Technologies SA, Paris, France). Los datos se han almacenado en un ordenador con Windows 7 (Microsoft Corporation, Washington, USA). 


\subsection{Desincronización de los ritmos alpha y beta}

En cada uno de los nueve electrodos considerados se estimó la densidad de potencia espectral (PSD) en ventanas solapadas de 1.5 segundos para las frecuencias entre 2 y $30 \mathrm{~Hz}$, con paso de $1 \mathrm{~Hz}$, empleando el método de Welch (ventanas de Hamming, $50 \%$ solapamiento). Se calculó la desincronización (ERD) de las señales en las bandas de frecuencia alpha $(7-13 \mathrm{~Hz})$ y beta $(13-30 \mathrm{~Hz})$, dentro de los períodos de Preparación del movimiento y de Movimiento (véase Figura 1), como el descenso estadísticamente significativo del valor de la potencia en esas ventanas respecto del valor de la potencia en la ventana temporal de Relax. El fenómeno ERD, para cada sujeto, canal, banda y lado del movimiento, se ha caracterizado mediante el valor medio en los 15 ensayos de un conjunto de variables en estos dos períodos.

Se ha analizado el fenómeno ERD en relación a tres segmentos temporales dentro de los dos períodos mencionados: a) ANT coincide con el período de Preparación del movimiento $(-5 \mathrm{~s}, 0)$; b) ANT-MOV, cuando el fenómeno se inicia en el período de Preparación del movimiento y se prolonga ininterrumpidamente al período de Movimiento; y c) MOV es período de Movimiento (0, 2s).

Las variables que caracterizan el fenómeno ERD, dependiendo del segmento temporal en que se localice son: 1) ERD Min, que representa el valor mínimo de ERD para cada uno de los segmentos temporales en los que se presente; 2) ERD Medio, que representa el valor medio de ERD para cada uno de los segmentos temporales en los que se presente; y 3) Porcentaje de tiempo que ocupa el fenómeno ERD dentro del segmento ANT o MOV; y 4) Duración en el tiempo del fenómeno ERD dentro del segmento ANT-MOV.

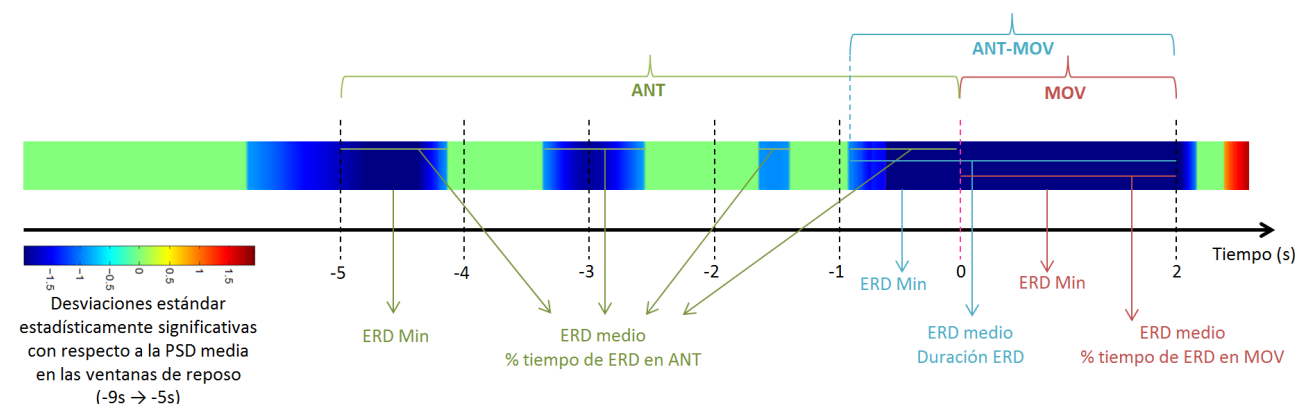

Fig. 2 Variables estructurales y temporales del fenómeno ERD durante la tarea de extensión de muñeca .

En la Figura 2 se muestra un ejemplo esquemático de la variación de la potencia de la señal en los tres segmentos temporales (ANT, ANT-MOV y MOV) y de las variables asociadas al fenómeno ERD para cada segmento para la tarea de extensión de muñeca. El valor de 
ERD en cada instante de tiempo es la potencia relativa a la potencia media en el período de Relax o basal.

Se han calculado también las diferencias entre las medias de cada una de las variables ERD del grupo experimental antes y después de la intervención de neuromodulación endógena y las medias de las variables ERD del grupo de control sano.

\subsection{Análisis estadístico}

Para cada variable extraída relacionada con el ERD se examinó la significación de la diferencia entre sus valores en el la etapa post-neuromodulación y pre-neuromodulación mediante un test $\mathrm{t}$ de medidas pareadas. Se utilizó el test de Levene para comprobar la igualdad de varianzas entre las poblaciones post y pre comparadas. En caso de no poder asumir igualdad de varianzas, se utilizó un test $\mathrm{t}$ de Welch para corregir dicha desigualdad. Todos los análisis estadísticos se realizaron mediante SPSS versión 25.0 (SPSS inc., Chicago, IL, USA). Los análisis se realizaron en un intervalo de confianza del 95\%. Los valores de significación $\mathrm{p}$ por debajo de 0,05 se consideraron estadísticamente significativos.

\section{Resultados}

Las Tablas 1 y 2 muestran los canales que han presentado cambios significativos de las variables del fenómeno ERD por la intervención de neuromodulación endógena en la mano derecha e izquierda, respectivamente. Se indica en negrita el nombre de la variable seguido entre paréntesis por la banda de frecuencia afectada. Debajo de cada variable, se presentan dos símbolos. El primero de ellos, $>$ ó <, representa si la variable ha incrementado o decrementado su valor, respectivamente, después de la intervención. El segundo, $\rightarrow$ ó $\leftarrow$, representa si el valor de la variable después de la intervención se aleja o se aproxima al valor medio de esa misma variable en los sujetos de control sanos.

Del análisis efectuado, se observa que se registran más cambios en el fenómeno ERD en ambas ventanas para la mano derecha, que es la mano dominante. Para esta mano, la mayor parte de los cambios se localizan en la corteza somatosensorial (CP3, CPz) y motora contralateral central (C3). En esta mano, todos los cambios en la corteza central $(\mathrm{Cz}, \mathrm{CPz}) \mathrm{e}$ ipsilateral (C4, CP4) se acercan a los valores medidos en los controles sanos. Estos cambios indican una menor desincronización en ambos ritmos alpha y beta, lo que apunta a una menor bilateralización de este fenómeno con la mano dominante tras la intervención de neruomodulación endógena. Los cambios se detectan en el período de Preparación del 
movimiento y se prolongan al período de Movimiento (ANT-MOV) y en el propio período de Movimiento (MOV).

Con respecto a la mano no dominante, la mayoría de los cambios tienen lugar en los segmentos temporales dentro del período de Preparación del movimiento (ANT y ANTMOV) y todos ellos indican una aproximación a los valores de los controles sanos. El tiempo de duración del fenómeno ERD se incrementa central y bilateralmente en ambas bandas de frecuencia en ese período. En la corteza prefrontal $(\mathrm{FCz})$, representando el área suplementaria motora, la desincronización en la banda beta aumenta acercándose a los controles sanos.

Tabla 1. Cambios estadísticamente significativos obtenidos en las variables neurofisiológicas relativas al fenómeno ERD en la mano DERECHA tras la intervención de neuromodulación endógena

\begin{tabular}{lcccccc}
\hline Mano derecha & C3 & CP3 & Cz & CPz & C4 & CP4 \\
\hline & $\begin{array}{c}\text { ERD Min } \\
\text { (alpha) }\end{array}$ & $\begin{array}{c}\text { ERD Medio } \\
\text { (alpha) }\end{array}$ & & & & \\
ANT-MOV & $<\rightarrow$ & & ERD Medio & & \\
& $>\leftarrow$ & ERD Min & & (beta) & & \\
& $($ alpha) & & $<\leftarrow$ & & \\
\hline & $<\rightarrow$ & & & & \\
MOV & \% ERD & \% ERD & \% ERD & ERD Medio & \% ERD \\
& & (beta) & (alpha) & (alpha) & (beta) & (alpha) \\
& & $<\rightarrow$ & $<\leftarrow$ & $<\leftarrow$ & $<\leftarrow$ & $<\leftarrow$ \\
\hline
\end{tabular}

Tabla 2. Cambios estadísticamente significativos obtenidos en las variables neurofisiológicas relativas al fenómeno ERD en la mano IZQUIERDA tras la intervención de neuromodulación endógena

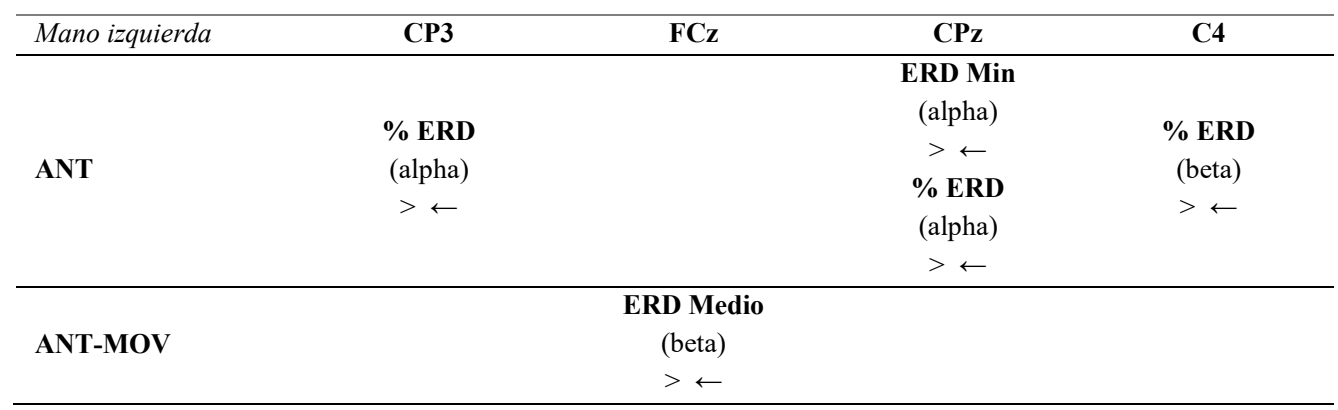




\section{Conclusiones}

La neuromodulación endógena efectivamente produce cambios sobre el fenómeno de la desincronización de los ritmos motores. En la mano dominante conduce a una menor desincronización relacionada con el movimiento en el hemisferio ipsilateral. En la mano no dominante produce un aumento en la duración de este fenómeno en el período de preparación del movimiento.

\section{Limitaciones del estudio}

El estudio realizado presenta limitaciones. La primera es que el tamaño de la muestra es relativamente pequeño. Los resultados podrían haber sido más significativos con un grupo mayor de pacientes con EP. La segunda limitación es la heterogeneidad que se presenta en la medicación, ya que los pacientes toman diferentes tipos de fármacos dependiendo de la estrategia terapéutica perseguida con el tratamiento. Todos los pacientes realizaron el estudio habiendo ingerido la medicación dopaminérgica pautada y encontrándose en el estado óptimo de influencia de la misma en las dos etapas del estudio, antes y después de la intervención de neuromodulación endógena, lo que necesariamente afecta a las variables neurofisiológicas medidas además de, posiblemente, a otros mecanismos corticales sensibles que no son tenidos en cuenta en este estudio.

\section{Agradecimientos}

El trabajo presentado forma parte de las investigaciones llevadas a cabo dentro de los proyectos NEUROMOD (DPI2015-68664-C4-1-R), MD (PIE-201650E055) y RoboCity2030-DIH-CM, Madrid Robotics Digital Innovation Hub, S2018/NMT-4331, financiado por "Programas de Actividades I + D en la Comunidad de Madrid" y cofinanciado por los Fondos Estructurales de la UE, habiendo sido financiado por ellos.

\section{Referencias}

Chung, JW., Burciu, RG., Oforic, E., Coombes, SA., Christou, EA., Okun, MS., Hess, CW., Vaillancour, DE. (2018). "Beta-band oscillations in the supplementary motor cortex are modulated by levodopa and associated with functional activity in the basal ganglia" en NeuroImage: Clinical, vol. 19, p. 559-571.

Erickson-Davis, CR., Anderson, JS., Wielinski, CL., Richter, SA., Parashos, SA. (2012). "Evaluation of neurofeedback training in the treatment of Parkinson's disease: A pilot study" en Journal of Neurotherapy, vol. 16, p. 4-11. 
Esmail, S., Linden, D. (2014). "Neural Networks and Neurofeedback in Parkinson's Disease" en NeuroRegulation, vol. 1, issue 3-4, p. 240-272.

Fahn, S. (2006). "A new look at levodopa based on the ELLDOPA study" en J. Neural Transm. Suppl. p. 419-26.

Fahn, S. (2011) “Classification of movement disorders”. En Mov. Disord. vol. 26, p. 947-57.

Fumuro, T., Matsuhashi, M., Mitsueda, T., Inouchi, M., Hitomi, T, Nakagawa, T., Brown, P. (2013). "Bereitschaftspotential augmentation by neuro-feedback training in Parkinson's disease" en Clinical Neurophysiology, vol. 124, p. 1398-1405.

Gulberti, A., Moll, C.K.E., Hamel, W., Buhmann, C., Koeppen, J.A. , Boelmans, K., Zittel, S., Gerloff, C., Westphal, M., Schneider, T.R., Engela, A.K. (2015). "Predictive timing functions of cortical beta oscillations are impaired in Parkinson's disease and influenced by L-DOPA and deep brain stimulation of the subthalamic nucleus" en NeuroImage: Clinical, vol. 9, p. 436-449.

Heida, T., Poppe, NR., de Vos, CC., van Putten, MJ., van Vugt, JP. (2014). "Event-related mu-rhythm desynchronization during movement observation is impaired in Parkinson's disease" en Clinical Neurophysiology, vol. 125, issue 9, p.1819-25.

Hutchison, WD., Dostrovsky, JO., Walters, JR., Courtemanche, R., Boraud, T., Goldberg, J., Brown, P. (2004). "Neuronal oscillations in the basal ganglia and movement disorders: evidence from whole animal and human recordings" en J. Neurosci. vol. 24, p. 9240-9243.

Kondylis, ED., Randazzo, MJ., Alhourani, A., Lipski, WJ., Wozny, TA., Pandya, Y., Ghuman, AS., Turner, RS., Crammond, DJ., Richardson, RM. (2016).” Movement-related dynamics of cortical oscillations in Parkinson's disease and essential tremor" en Brain, vol. 139, p. 2211-2223.

Moisello, C., Blanco, D., Lin, J., Panday, P., Kelly, SP., Quartarone, A. (2015). "Practice changes beta power at rest and its modulation during movement in healthy subjects but not in patients with Parkinson's disease" en Brain and Behavior, vol. 5, issue 10.

Mostile, G., Nicoletti, A., Dibilio, V., Luca, A., Pappalardo, I., Giuliano, L., Cicero, CE., Sciacca, G., Raciti, L., Contrafatto, D., Bruno, E., Sofia, V., Zappia, M. (2015). "Electroencephalographic lateralization, clinical correlates and pharmacological response in untreated Parkinson's disease" en Parkinsonism Relat Disord., vol. 21, issue 8, p. 948-53.

Neuper, C., Wörtz, M., Pfurtscheller, G. (2006). "ERD/ERS patterns reflecting sensorimotor activationand deactivation" en Neuper \& Klimesch (Eds.) Progress in Brain Research, vol. 159.

Pfurtscheller, G., Lopes da Silva, FH. (1999). "Event-related EEG/MEG synchronization and desynchronization: basic principles” en Clinical Neurophysiology, vol. 110, p.1842-1857.

Sharott, A., Gulberti, A., Zittel, S., Tudor Jones, AA., Fickel, U., Munchau, A., Koppen, JA., Gerloff, C., Westphal, M., Buhmann, C., Hamel, W., Engel, AK., Moll, CKE. (2014). “Activity parameters of subthalamic nucleus neurons selectively predictmotor symptom severity in Parkinson3s disease" en $J$. Neurosci., vol. 34, issue 18, p. 6273-6285.

Subramanian, L., Hindle, JV., Johnston, S., Roberts, MV., Husain, M., Goebel, R., Linden, D. (2011). "Real-time functional magnetic resonance imaging neurofeedback for treatment of Parkinson's disease" en Journal of Neuroscience, vol. 31, p. 16309-16317. 


\title{
Estrategia para el control háptico, basado en electromiografía, de un exoesqueleto de mano para neurorehabilitación
}

\author{
Cisnal, Ana ${ }^{\text {a }}$ Moreno, Victor ${ }^{\mathrm{a}}$; Pérez-Turiel, Javier ${ }^{\mathrm{a}}$; Alonso, Rubén ${ }^{\mathrm{b}}$; Fraile- \\ Marinero, J.Carlos ${ }^{a}$ y Lobo, Victor ${ }^{a}$ \\ ${ }^{a}$ Grupo de Robótica Médica, Instituto de las Tecnologías Avanzadas de la Producción (ITAP), Univ. \\ de Valladolid, España. ana.cisnal@hotmail.com, turiel@eii.uva.es \\ b Área de Bienestar y Salud, División de Sistemas, Fundación Cartif, Parque Tecnológico de Boecillo, \\ Boecillo, Valladolid, España. rubalo@cartif.es
}

\begin{abstract}
RobHand is a neuro rehabilitation system for the hand and wrist of subjects who have suffered a stroke and must recover the mobility lost. It includes a hand exoskeleton, an interface with the patient for performing therapies based on a virtual environment and a management system for the information associated with patients and therapies. In this paper we present the approach and the development status of the control architecture of the hand exoskeleton integrated in the rehabilitation system. Their kinematic and dynamic models, and the sEMG based bio-operative controller that implements active-assisted therapies tailored to the patient are described.
\end{abstract}

Keywords: rehabilitation, robotics, exoskeletons, modeling, control, sEMG, biosignals.

\section{Resumen}

RobHand es un sistema de neuro rehabilitación para la mano y muñeca de sujetos que han sufrido un accidente cerebrovascular y deben recuperar la movilidad perdida como consecuencia del mismo. Incluye un exosqueleto de mano, un interfaz con el paciente para la realización de terapias basadas en un entorno virtual y un sistema de gestión de toda la información asociada a los pacientes y las terapias realizadas. En este trabajo presentamos el planteamiento y el estado de desarrollo de la arquitectura de control del exoesqueleto de mano integrado en el sistema de rehabilitación. Se describen sus modelos cinemático y dinámico, y el controlador biocooperativo basado en sEMG para implementar terapias activas-asistidas a medida del paciente.

Palabras clave: rehabilitación, robótica, exoesqueletos, modelado, control, sEMG, bioseñales. 


\section{Introducción}

RobHand es un sistema de neuro rehabilitación para la mano y muñeca de sujetos que han sufrido un accidente cerebrovascular y deben recuperar la movilidad perdida como consecuencia del mismo (Ukei 2012). El sistema incluye un exosqueleto de mano (Fig.1), un módulo de muñeca, un interfaz con el paciente para la realización de terapias basadas en un entorno virtual y un sistema de gestión de toda la información asociada a los pacientes y las terapias realizadas.
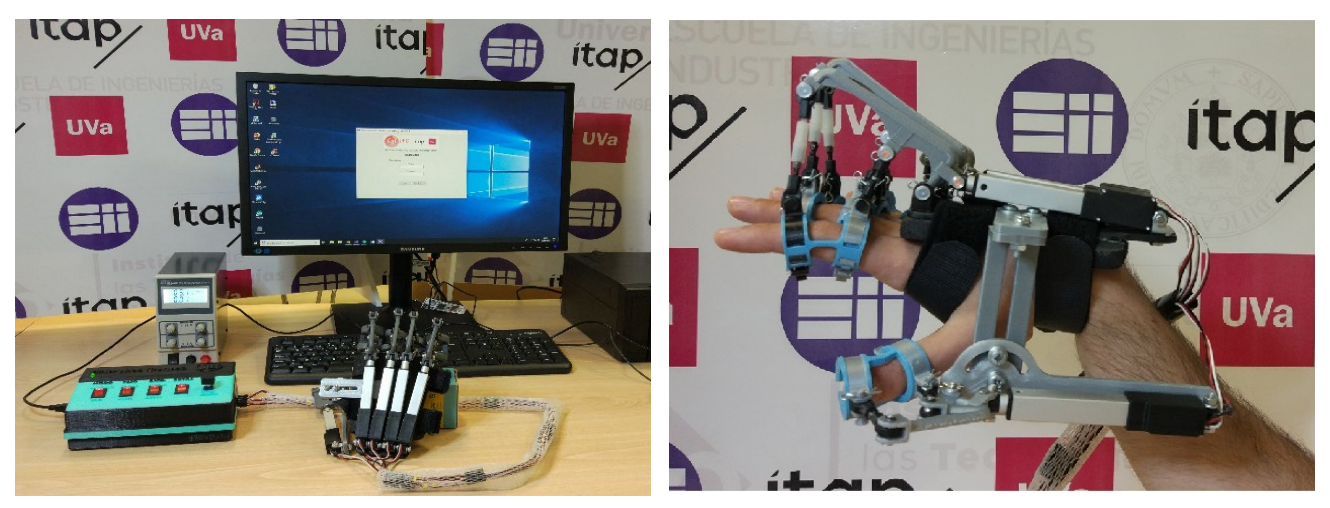

Fig. 1 Componentes de la plataforma RobHand (a) y vista del Exoesqueleto de mano (b)

Es un desarrollo financiado por el CDTI y se lleva a cabo en colaboración por la empresa CyL ImasD Informática (Salamanca), el grupo de Robótica Médica del ITAP (UVa) y el servicio de rehabilitación del Hospital Clínico Universitario (HCU) de Valladolid. El sistema permite realizar tanto terapias de tipo pasivo, en los cuales el sujeto no ejerce ninguna fuerza y toda la responsabilidad es del sistema, como otras de tipo activo asistido en las que se busca conseguir un comportamiento de tipo asistencia a medida (assist as needed) en el que el sistema detecta la fuerza realizada por el paciente y la complementa hasta el nivel de desempeño deseado.

El objetivo principal del presente trabajo es presentar el planteamiento y el estado de desarrollo de la arquitectura de control del dispositivo de rehabilitación. Este adopta un esquema jerárquico en tres niveles de tal modo que el nivel superior, L1, gestiona la ejecución de las terapias virtuales y adapta dinámicamente el comportamiento del sistema al estado de desempeño del paciente. El nivel inferior L3 se ocupa del control de los grados de libertad del dispositivo y se implementa directamente mediante los drivers de los actuadores. Entre ambos, el nivel intermedio L2 implementa una estrategia de interacción háptica con control de admitancia. Para ello planteamos utilizar registros de electromiografía de superficie del paciente para estimar la intencionalidad de movimiento, y la lectura del consumo de corriente de los motores, para adaptar dinámicamente el nivel de asistencia a la situación del paciente. Se ha desarrollado un hardware específico para el 
registro y tratamiento de las señales de EMG y los algoritmos de control se ejecutan en un DSP para favorecer la autonomía del dispositivo. En la actualidad se están realizando pruebas de las terapias pasivas con 5 pacientes en el HCU y en breve comenzarán las pruebas con terapias activas.

El resto del documento se estructura de la siguiente forma. Los apartados 2 y 3 describen la arquitectura de control y la tipología de terapias soportadas por la plataforma, respectivamente. En el apartado 4 se detallan los aspectos más relevantes de la estrategia de control que se aplica en las terapias activas: obtención de los modelos cinemático y dinámico y control bio-cooperativo basado en el procesamiento de las señales de sEMG. Finalmente, el apartado 6 resume las conclusiones del trabajo.

\section{Arquitectura de control del dispositivo}

La estrategia de control planteada, en el caso de las terapias activas, busca implementar el paradigma "Assist as Needed" mediante la detección de la "intención de movimiento" del paciente (Yap, 2016), midiendo la actividad electromiográfica de los músculos del brazoantebrazo implicados en el movimiento de sus dedos. La arquitectura diseñada para el control del exoesqueleto se muestra en la figura 2.

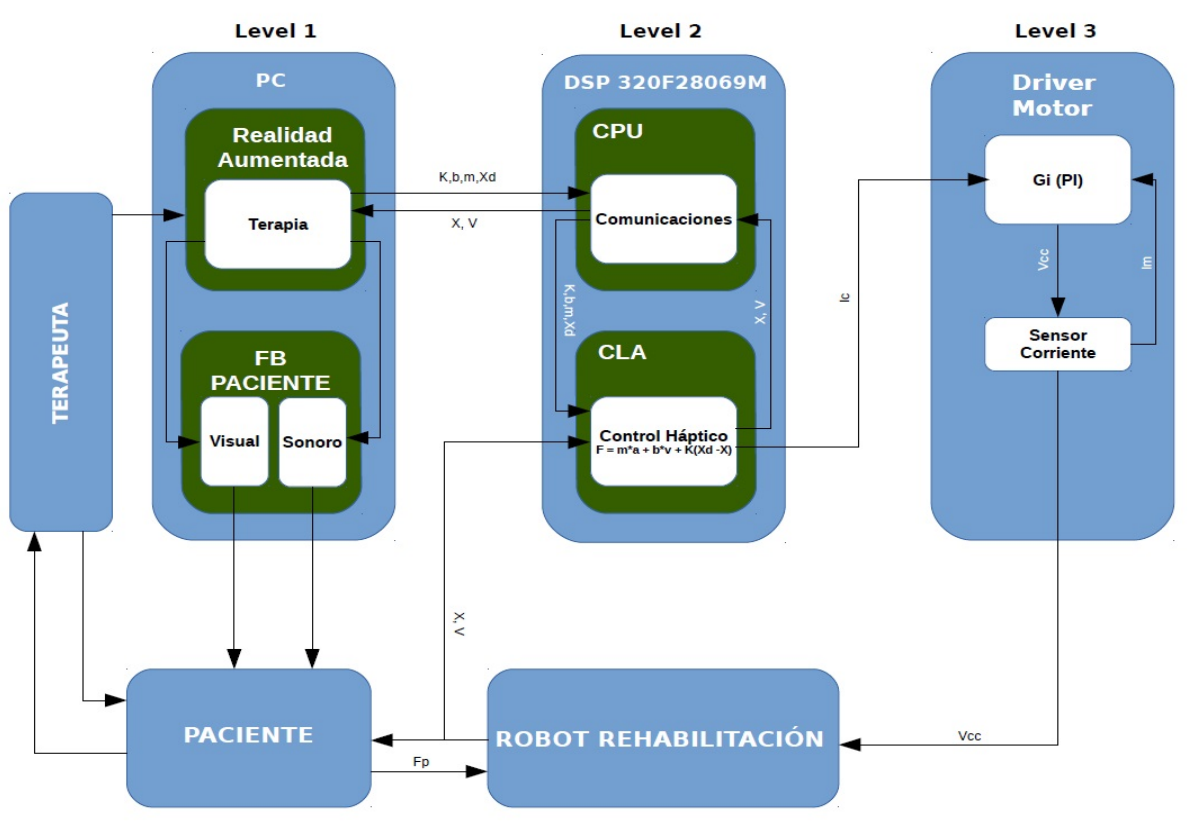

Fig. 2 Arquitectura de control de la plataforma RobHand 
El sistema de control adopta un esquema jerárquico en tres capas, buscando la modularidad del diseño y facilitar su adaptación a nuevos requisitos (Cisnal 2018):

- La capa L1 constituye el nivel de control superior, y se encarga de gestionar la ejecución de las terapias virtuales y adapta dinámicamente el comportamiento del sistema al estado de desempeño del paciente. Las terapias diseñadas se basan en el concepto de "serious game" (figura 3), ya que se consideran más efectivas que las terapias tradicionales porque aumentan la motivación de los pacientes, siendo más probable que los pacientes aumenten el tiempo dedicado a la rehabilitación (Cameirao, 2007)].

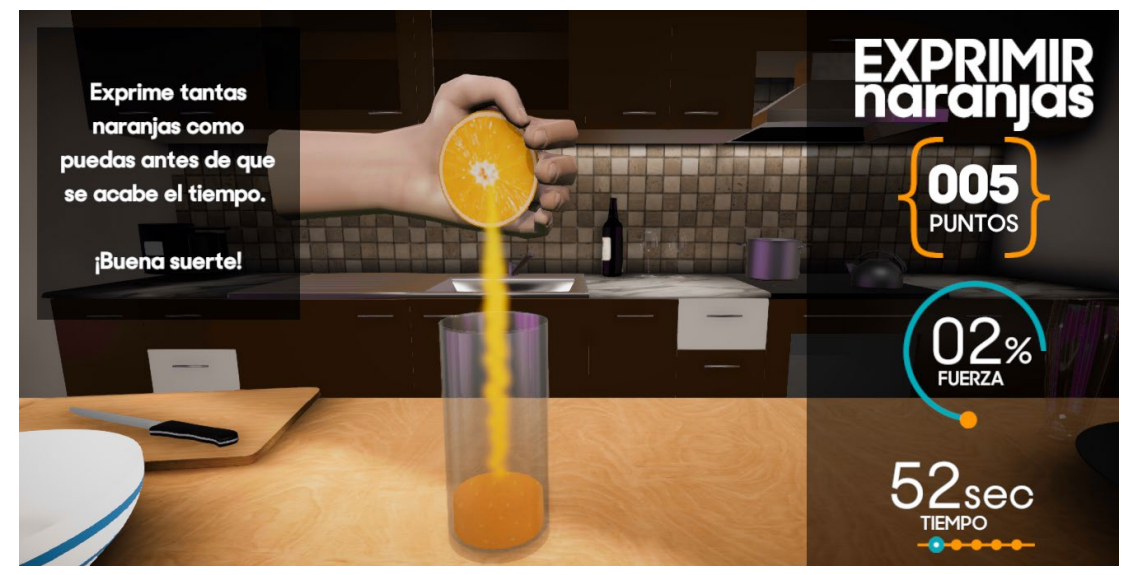

Fig. 3. Uno de los entornos virtuales para la realización de terapias de rehabilitación.

- El nivel intermedio, L2, implementa una estrategia de interacción háptica con control de admitancia. Para ello utilizamos registros de electromiografía de superficie (sEMG) del paciente para estimar la intencionalidad de movimiento, y la lectura del consumo de corriente de los motores, para adaptar dinámicamente el nivel de asistencia a la situación del paciente (Lucas, 2011). Su implementación se realiza en la placa de evaluación LaunchXL-F28069M (Texas Instruments), que incorpora el microcontrolador TMS320F28069M cuya CPU puede alcanzar hasta $90 \mathrm{MHz}$ de ciclo de reloj, cuenta con CLA (Control Law Accelerator) y permite controlar múltiples módulos como ePWM, ADC, SCI, SPI....

- El nivel inferior L3 realiza el control de los grados de libertad del dispositivo y se implementa directamente mediante los drivers de los actuadores (en nuestro caso actuadores lineales L2-30-100-6-I de Actuonix). El actuador cuenta con tres tipos de control de posición y de una señal de realimentación de posición. Este nivel incorpora también la electrónica necesaria para adecuar la señal de control a los actuadores y para medir la corriente consumida por estos. 


\section{Tipología de terapias soportadas por la plataforma.}

La plataforma RobHand soporta tres tipos de terapias en función del grado y tipo de participación del paciente: terapias pasivas, terapias bilaterales y terapias activas (SandovalGonzález, 2016). En las terapias bilaterales y activas se plantea una estrategia biocooperativa, es decir, el controlador considera la intencionalidad de movimiento del sujeto a partir del registro y procesado de las señales electromiográficas (sEMG) de los músculos del antebrazo implicados en el movimiento de apertura y cierre de la mano

\subsection{Terapias pasivas}

Las terapias pasivas son ejercicios de rehabilitación programados, basadas en la repetición de movimientos que implican la flexión y extensión de los dedos. Las terapias pasivas disponibles son las siguientes:

- Abrir y cerrar la mano: flexion y extension de los cinco dedos de la mano de manera simultánea.

- Apertura y cierre de los dedos: flexión y extensión de los dedos de la mano de manera individual

- Pinza fina: flexión y extensión del dedo pulgar y del dedo índice de manera simultánea, de tal manera que se logra un agarre de tipo pinza.

- Pinza guresa: flexión y extensión del dedo pulgar contra el resto de los dedos de manera simultánea, de tal manera que se logra un agarre de tipo pinza gruesa.

El software de gestión de la plataforma permite definer parámetros característicos de la terapia como la velocidad y el grado de apertura y cierre de la mano, el número de repeticiones y el tiempo de espera entre estas, de manera que la terapia puede adecuarse a las necesidades del paciente.

\subsection{Terapias bilaterales (espejo)}

Las terapias bilaterales, también llamadas terapias espejo, se basan en replicar el movimiento de referencia que reproduce la mano sana en el robot de rehabilitación, con el bojetivo de asistir a la mano insana (Ueki, 2012).

El movimiento de referencia se determina a partir de las señales EMG de los músculos extensor digitorum communis (ED) y flexor carpi ulnaris (FU) del antebrazo de la mano sana, que son dos músculos responsables de la apertura y cierre de la mano, respectivamente. 
La determinación del movimiento de referencia requiere de la normalización de las señales sEMG (Lehman, 2013). El cálculo de la señal normalizada implica el filtrado y la rectificación de la señal mediante el cálculo de la envolvente mediante el uso de la transformada de Hilbert en el dominio del tiempo y su normalización respecto al valor máximo de las contracciones voluntarias (Maximum Voluntary Contraction, MVC).

Las terapias bilaterales utilizan un control de tipo binario, también conocido como control por umbrales o de tipo ON/OFF. Para ello se definen tres estados posibles del exoesqueleto en función de los valores que tomen los valores MVC de las señales sEMG de los músculos ED y FU (ver figura 4). Los tres posibles estados son reposo, cierre y apertura, y se definen de la siguiente manera.

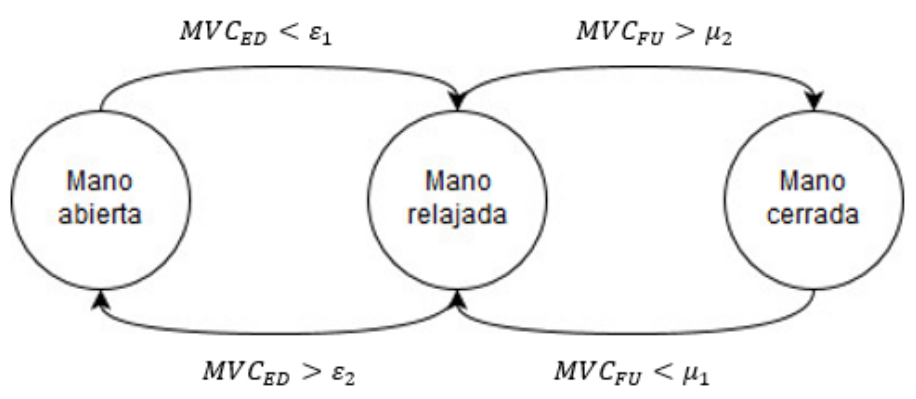

Fig. 4. Estados que puede adoptar RobHand en función de las señales EMG de los músculos ED y FU del sujeto.

Siendo $\mu_{1}$ y $\varepsilon_{1}$ los valores correspondientes a la desactivación de la actividad musculares de los músculos FU y ED, respectivamente. Estos valores, junto a otros dos umbrales correspondientes a la activación muscular máxima $\left(\begin{array}{lll}\mu_{2} & \text { y } & \varepsilon_{2}\end{array}\right)$ son obtenidos mediante un proceso de calibración propio para cada sujeto.

\subsection{Terapias activas}

Las terapias activas se controlan mediante una estrategia que implementa el paradigma "Assist as Needed" con el objetivo de proporcionar la asistencia necesaria al paciente para realizar con éxito las terapias. La arquitectura diseñada para el control de un subconjunto que corresponde a un dedo del exoesqueleto de mano se muestra en la figura 5.

La capa de control L1 recibe la posición actual del exoesqueleto y mediante un modelo de impedancia proporciona a la capa L2 la consigna de fuerza que tiene que realizar el conjunto exoesqueleto-paciente para llegar a la posición objetivo establecido por la terapia en cada momento. 
La capa de control intermedia L2 calcula la señal de control del actuador (L3) mediante un modelo de admitancia, cuya entrada es el error de fuerza, calculado como la fuerza del conjunto menos la fuerza realizada por el usuario y por el actuador.

La fuerza realizada por el actuador se determina mediante un sensor de corriente, conocida la relación lineal entre la fuerza y la corriente $(\mathrm{K})$, evitando de esta manera la incorporación de sensores de fuerza en el exoesqueleto (Pinhas, 2015).

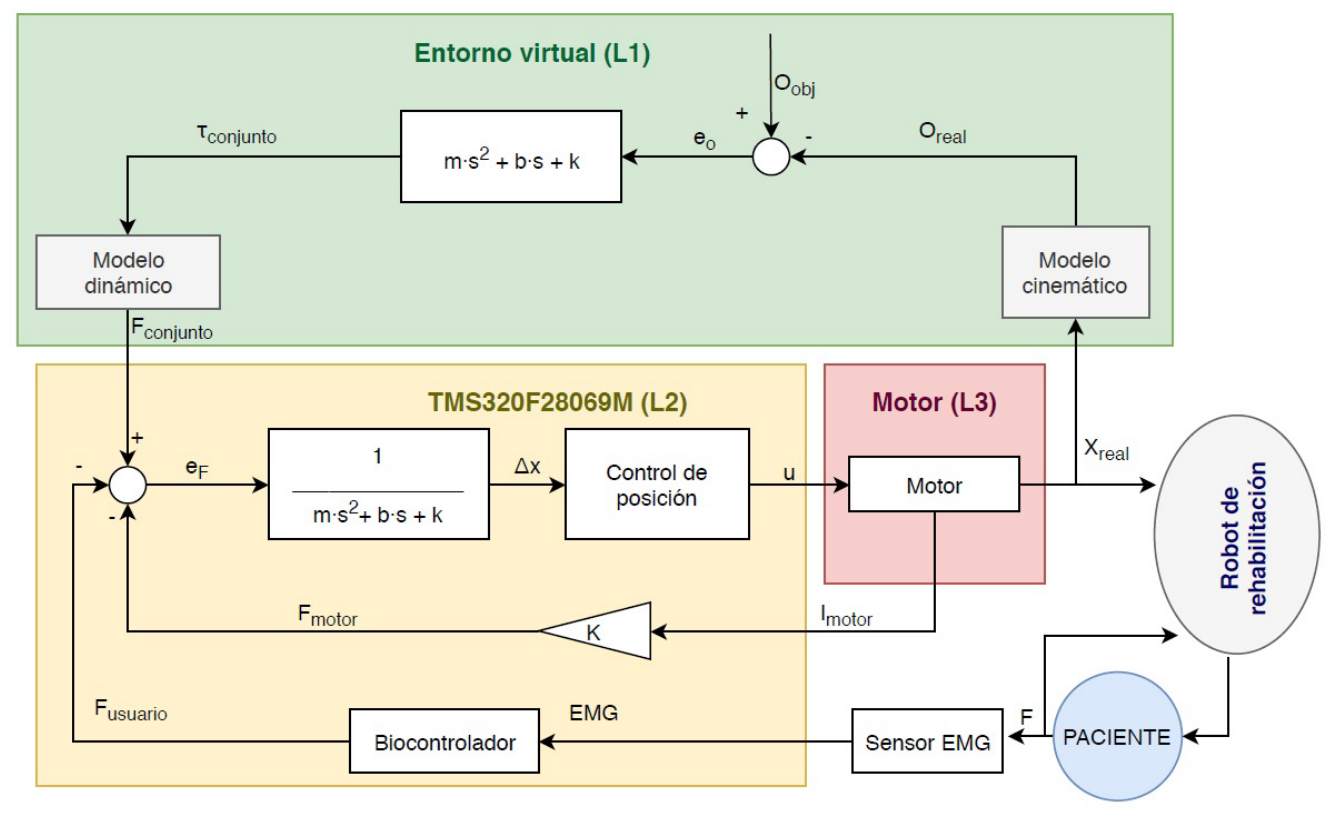

Fig. 5. Diagrama de bloques del control para terapias activas de RobHand.

La fuerza realizada por el usuario se estima mediante el registro de las señales sEMG de los ED y FU. El bio-controlador tiene como objetivo la normalización de las señales sEMG, para posteriormente estimar la fuerza realizada por el usuario. La fuerza realizada por el usuario se estima considerando que las señales normalizadas de sEMG están relacionadas de manera lineal con la fuerza (Kuriki, 2013), mediante un modelo binario-proporcional.

\section{Terapias activas: estrategia de control}

Para poder realizar un correcto control del exoesqueleto es necesario conocer la posición de determinados elementos del mismo y las fuerzas que se ejercen entre estos y los dedos del paciente. Es decir, necesitamos conocer sus modelos cinemático y dinámico. Asimismo es fundamental estimar la fuerza ejercida por el paciente a partir del registro de sEMG . 


\subsection{Gestión del entorno virtual: Modelo cinemático.}

El diseño mecánico del exoesqueleto plantea un mecanismo por cada dedo, idéntico en todos, cambiando únicamente las distancias entre los puntos de rotación. Esto nos permite realizar el estudio sobre un dedo y después extrapolarlo al resto. Para ello elaboramos un modelo de alambre (figura 6), que nos facilite parametrizar las distancias, longitudes y ángulos, pudiendo así hacer un estudio genérico y luego particularizar a cada dedo.

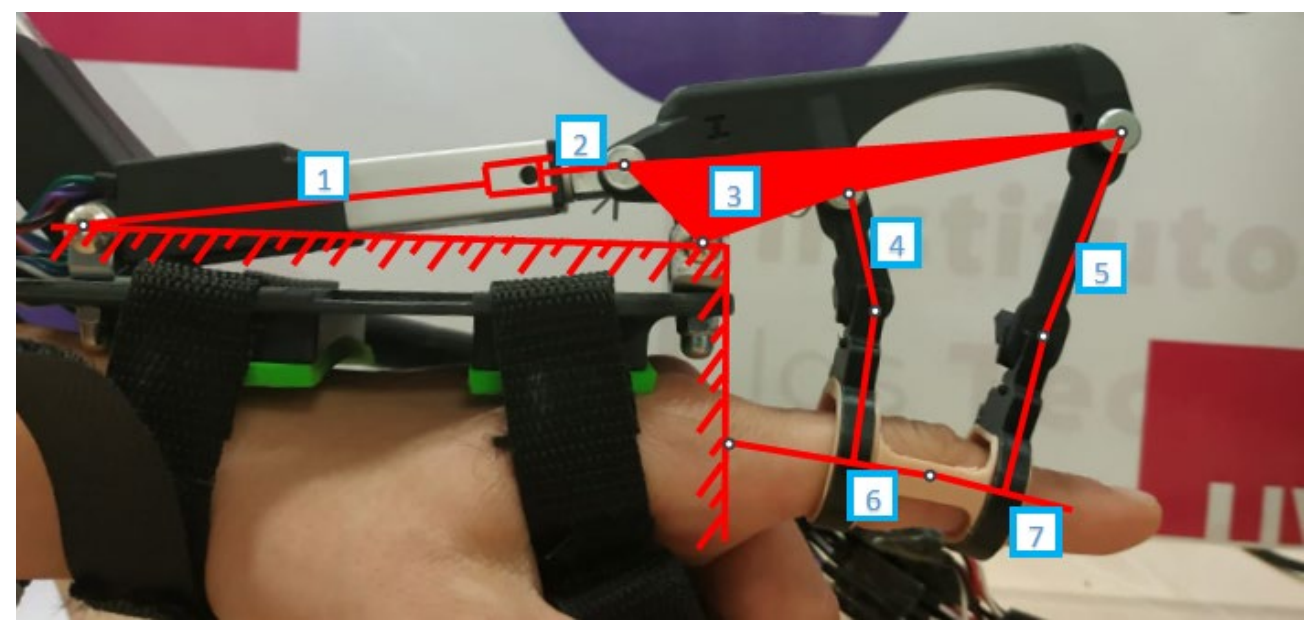

Fig. 6. Modelo de alambre de RobHand superpuesto a una imagen del exoesqueleto.

Centramos el estudio en los parámetros $\alpha$ y $\beta$, posición angular de la falange proximal respecto al metacarpo, y posición angular de la falange media respecto de la proximal. En ambos casos, serán valores positivos para la hiperextensión y negativos para la flexión. El método de Grübler nos indica que se trata de un mecanismo de un único grado de libertad, lo que nos permite fijar la posición del vástago del motor como variable independiente.

Para este estudio se ha utilizado el método de los anillos de resolución de mecanismos. Este consiste en definir anillos cerrados en el mecanismo y trabajar con la ecuación que define ese anillo para obtener la velocidad de cada falange. Para conocer la posición (figura 7) de cada falange resolvemos el sistema formado por las expresiones de cada anillo en los ejes:

Posición de la falange proximal " $\alpha$ ”:

$$
\begin{array}{lr}
\text { Eje } \mathrm{x}: & \bar{l}_{2}+\left(\bar{l}_{4}-\bar{l}_{5}\right) \cos \alpha+\bar{l}_{6} \sin q_{6}+\bar{l}_{7} \sin q_{7}-\bar{l}_{13} \cos \varphi=0 \\
\text { Eje y: } & -\bar{l}_{3}+\left(\bar{l}_{4}-\bar{l}_{5}\right) \sin \alpha+\bar{l}_{6} \cos q_{6}+\bar{l}_{7} \cos q_{7}-\bar{l}_{13} \sin \varphi=0
\end{array}
$$

Posición de la falange media " $\beta$ ":

Eje $\mathrm{x}: \bar{l}_{2}+\bar{l}_{4} \cos \alpha+\bar{l}_{9} \cos \beta-\bar{l}_{10} \sin q_{8}+\bar{l}_{11} \sin q_{11}-\bar{l}_{15} \cos q_{15}-\bar{l}_{13} \cos \varphi=0$

Eje y: $-\bar{l}_{3}+\bar{l}_{4} \sin \alpha+\bar{l}_{9} \sin \beta+\bar{l}_{10} \cos q_{8}+\bar{l}_{11} \cos q_{11}-\bar{l}_{15} \sin q_{15}-\bar{l}_{13} \sin \varphi=0$ 

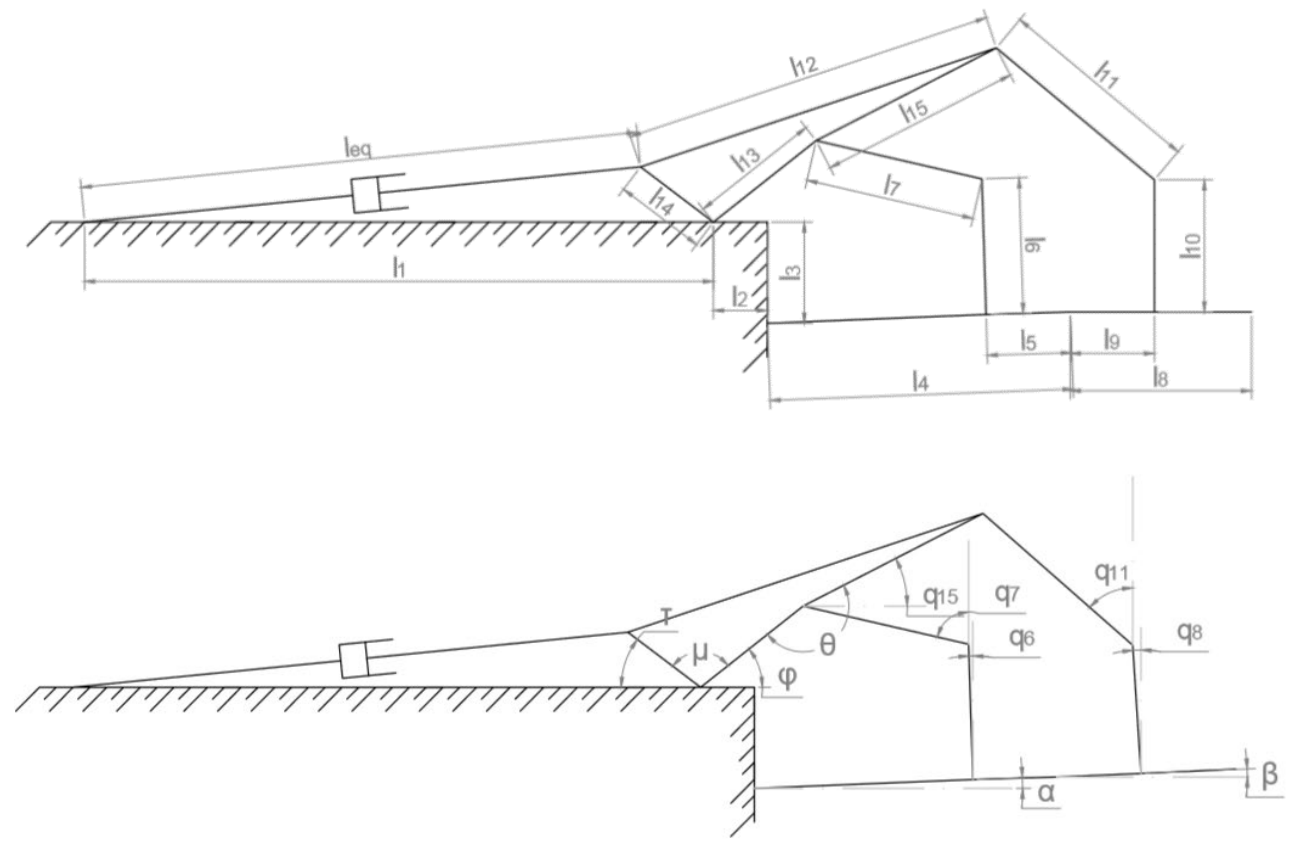

Fig. 7. Parametrización de ángulos y longitudes de un dedo del exoesqueleto.

Desarrollando el modelo cinemático, cuya obtención detallada se puede ver en (Cisnal, 2018), obtenemos las ecuaciones que nos permiten hallar la velocidad de rotación de las falanges proximal (5) y media (6):

$$
\begin{aligned}
& \dot{\alpha}=\frac{\dot{\varphi}}{l_{45} \cos \left(\alpha-q_{7}\right)}\left(l_{13} \sin q_{7} \sin \varphi+l_{13} \cos q_{7} \cos \varphi\right) \\
& \dot{\beta}=\frac{\dot{\varphi}}{l_{9} \cos \left(\beta-q_{11}\right)}\left(l_{13} \sin q_{11} \sin \varphi+l_{13} \cos q_{11} \cos \varphi\right)
\end{aligned}
$$

Donde tenemos que la variable $\varphi$ es la que depende directamente de la posición del motor. Así, tenemos:

$$
\varphi=\pi-\mu-\operatorname{acos}\left(\frac{l_{14}{ }^{2}+l_{1}{ }^{2}-L_{e q}{ }^{2}}{2 l_{14} l_{1}}\right) ; \quad \dot{\varphi}=\frac{L_{e q} L_{e q}}{l_{14} l_{1} \sqrt{1-\left(\frac{l_{14}{ }^{2}+l_{1}{ }^{2}-L_{e q}{ }^{2}}{2 l_{14} l_{1}}\right)^{2}}}
$$

Este modelo cinemático ha sido validado usando el software "Kinovea 0.8.26" de análisis biomecánico. Con el se ha calculado el error entre los datos generados por el modelo cinemático y los arrojados por la validación, y resultan ser menores del $6.5 \%$, lo que se considera una aproximación aceptable para nuestros objetivos. 


\subsection{Gestión del entorno virtual: Modelo dinámico.}

Para nuestra aplicación, el modelo dinámico debe permitirnos conocer una fuerza aplicada sin necesidad de conocer todas las fuerzas de unión entre las piezas; debe permitir introducir la posible resistencia que ofrezca el usuario al movimiento del exoesqueleto por pequeñas espasticidades; su matemática no debe ser compleja, para facilitar su implementación en el algoritmo de control, y debe ser invertible, permitiendo conocer la fuerza en el dedo a partir de la del motor y viceversa.

Teniendo en cuenta estos requisitos hemos evaluado como alternativas la conveniencia de utilizar Teoremas Vectoriales, Ecuaciones de Lagrange y el Método de las Potencias Virtuales, decantándonos por este último.

Así, su aplicación directa se traduce en la siguiente fórmula:

$$
\bar{F} \cdot \overline{v^{*}}+\overline{F^{\prime}} \cdot \overline{v^{*}}+\bar{M} \cdot \overline{\omega^{*}}+\overline{M^{\prime}} \cdot \overline{\omega^{*}}=0
$$

donde:

- $\quad \bar{F} \cdot \overline{v^{*}}$ es la potencia aportada por las fuerzas reales sobre el sistema.

- $\quad \overline{F^{\prime}} \cdot \overline{v^{*}}$ es la potencia aportada por las fuerzas de inercia sobre el sistema.

- $\bar{M} \cdot \overline{\omega^{*}}$ es la potencia aportada por los momentos externos sobre el sistema.

- $\overline{M^{\prime}} \cdot \overline{\omega^{*}}$ es la potencia aportada por los momentos debidos a la inercia del sistema.

Como una de las fuerzas que queremos conocer es la que el motor realiza sobre el exoesqueleto, debemos simplificar la unión del motor con el resto del mecanismo y considerar su fuerza como externa a este, con lo que obtenemos el siguiente esquema:

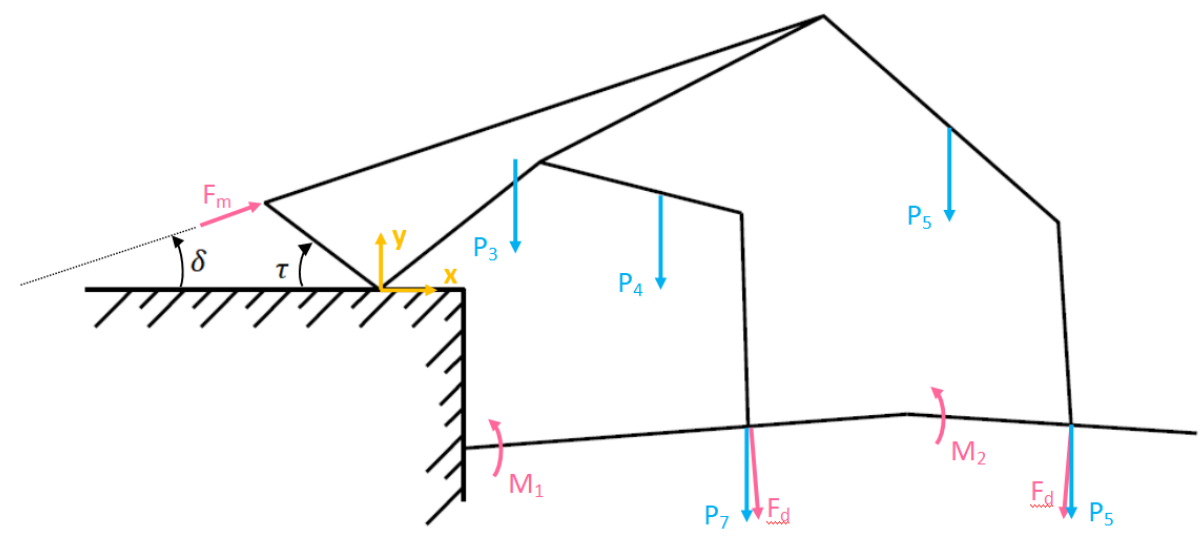

Fig. 8. Diagrama de fuerzas en un dedo. 
En el diagrama de la figura 8 aparecen una serie de fuerzas y momentos que representan:

- $\quad F_{m}$ - Fuerza Motor: fuerza que el motor ejerce sobre el mecanismo, se toma positiva para el movimiento de cierre del mecanismo.

- $\quad P_{x}$ - Peso del elemento "x": es el peso de cada uno de los elementos del sistema.

- $\quad F_{d 1}$ - Fuerza en el dedo: fuerza que el mecanismo ejerce sobre el dedo, en la falange proximal, se toma positiva cuando el mecanismo ayuda al cierre del dedo o se opone a la apertura.

- $\quad F_{d 2}$ - Fuerza en el dedo: fuerza que el mecanismo ejerce sobre el dedo, en la falange media, se toma positiva cuando el mecanismo ayuda al cierre del dedo o se opone a la apertura.

- $\mathrm{M}_{1}, \mathrm{M}_{2}$ - Momentos Usuario: son momentos que aparecen como resultado de las fuerzas que el usuario, de forma involuntaria, ejerce sobre el mecanismo en las falanges proximal y media respectivamente. Estos momentos pretenden recoger aquellas fuerzas que aparecen como consecuencias de posibles espasticidades residuales que el paciente tenga en el momento de la terapia.

En nuestro sistema de control de terapias activas únicamente necesitamos conocer la fuerza aplicada sobre la falange proximal. Además, como se trata de un mecanismo de un grado de libertad, las fuerzas $F_{d 1}$ y $F_{d 2}$ no son independientes entre sí, por lo que podemos simplificar el sistema para estudiar únicamente una de ellas. Por otra parte, este método trabaja con las fuerzas aplicadas sobre el sistema, por tanto en vez de $F_{d}$ debemos utilizar la que el dedo ejerce sobre el mecanismo, $F_{d}^{\prime}$, que tendrá el mismo modulo y dirección que $F_{d}$, pero sentido opuesto. Por último, debemos diferenciar entre el proceso de apertura y el de cierre ya que el sentido de algunas fuerzas no cambia, pero el de otras lo hace.

Analizando el proceso de apertura, obtenemos la siguiente ecuación (ver figura 9):

$$
F_{m} \cdot v_{m}-F_{d}^{\prime} \cdot v_{f d}-M_{1} \cdot \dot{\alpha}=0
$$

El valor de $v_{m}$ debe ser proporcionado por el rehabilitador, mientras que $\dot{\alpha}$ y $\mathrm{v}_{\mathrm{fd}}$ se obtienen del modelo cinemático $\left(\mathrm{v}_{\mathrm{fd}}\right.$ se calcula como $\mathrm{v}_{\mathrm{fd}}=\dot{\alpha}\left(\mathrm{l}_{4}-\mathrm{l}_{5}\right)$ ).

Así podremos utilizar como variable de entrada o salida indistintamente $F_{m}$ o $F_{d}$ para obtener la contraria:

$$
\begin{aligned}
& F^{\prime}{ }_{d}=F_{d}=\frac{1}{v_{f d}}\left(F_{m} \cdot v_{m}-M_{1} \cdot \dot{\alpha}\right) \\
& F_{m}=\frac{1}{v_{m}}\left(F_{d} \cdot v_{d}+M_{1} \cdot \dot{\alpha}\right)
\end{aligned}
$$




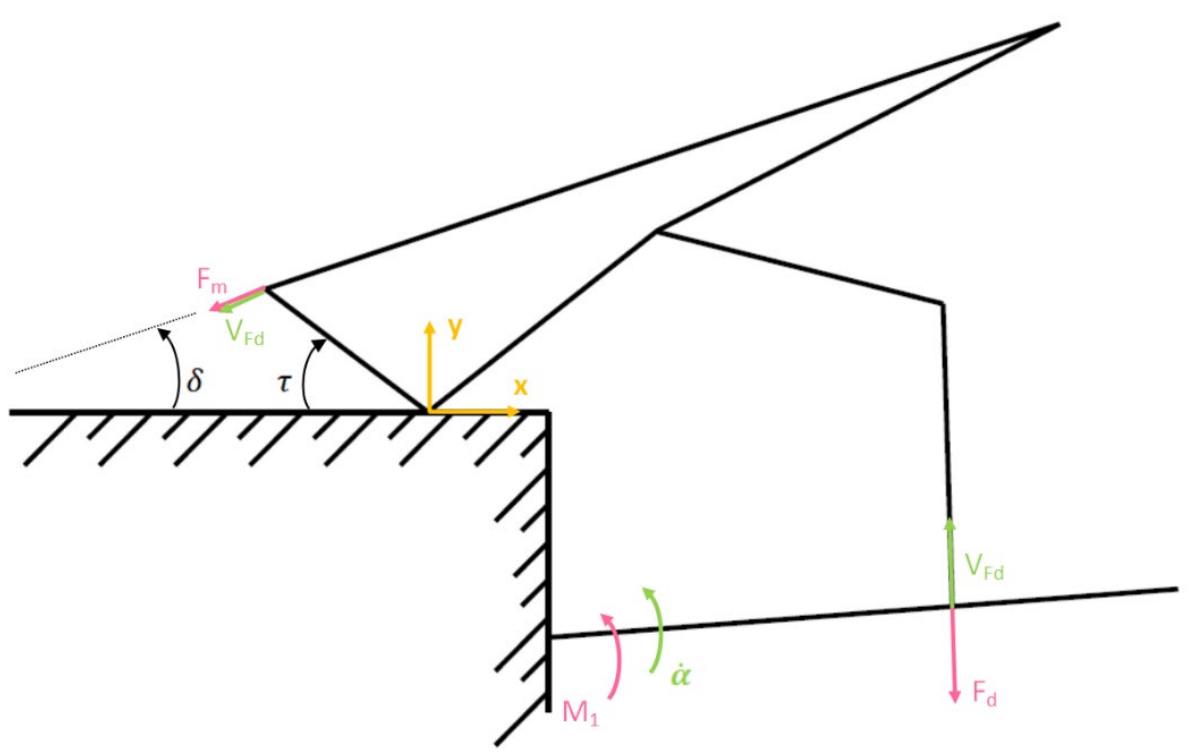

Fig. 9. Diagrama de fuerzas en apertura.

$\mathrm{Al}$ interpretar los resultados hay que tener en cuenta que $F_{d}^{\prime}$ no es la fuerza que el paciente va a percibir, si no que es la fuerza que se va a aplicar sobre el modelo teórico del "assist as needed". Tampoco $F_{d}$ es la fuerza percibida; la fuerza que el paciente notará será $F_{d}^{\prime \prime}$ que se deduce a continuación tienendo en cuenta las fuerzas aplicadas sobre el dedo. En este caso no se puede suponer un balance de fuerzas, pues el sistema se está desplazando, por tanto:

$$
F_{d}^{\prime \prime}=M_{1} \cdot l_{45}+F_{d}
$$

En el proceso de cierre hay que tener presente que las fuerzas del dedo y del motor, así como las velocidades presentes, cambian su sentido, con lo que obtenemos las siguientes ecuaciones:

$$
\begin{aligned}
& F_{m} \cdot v_{m}+M_{1} \cdot \dot{\alpha}-F_{d}^{\prime} \cdot v_{f d}=0 \\
& F_{d}^{\prime}=\frac{1}{v_{f d}}\left(F_{m} \cdot v_{m}+M_{1} \cdot \dot{\alpha}\right) \\
& F_{m}=\frac{1}{v_{m}}\left(F_{d} \cdot v_{d}-M_{1} \cdot \dot{\alpha}\right)
\end{aligned}
$$

Como en el proceso de apertura, en el proceso de cierre $F_{d}^{\prime}$ no es la fuerza percibida por el paciente. Esta, como en el caso anterior, se calcula:

$$
F_{d}^{\prime \prime}=F_{d}-M 1 \cdot l_{45}
$$


Las ecuaciones del modelo dinámico nos permiten calcular la fuerza combinada resultante de la interacción entre un dedo del paciente y el mecanismo correspondiente del exoesqueleto y generar, por tanto, la consigna para el controlador háptico de admitancia implementado en el módulo L2 de la arquitectura de control.

\subsection{Detección de intención. Control biocooperativo}

La estrategia de control biocooperativo se basa en detectar la intención de movimiento del paciente a través del tratamiento y análisis de las señales EMG de los músculos encargados del movimiento de apertura y cierre de la mano (Fleischer, 2006).

Estrategias de control como la aplicada en (Fraile, 2016) asumía la intención del paciente. Con el objetivo de que la cooperación humano-robot en las terapias de rehabilitación sea más eficiente, se implementa un control biocoperativo para conseguir un control de movimientos fiable.

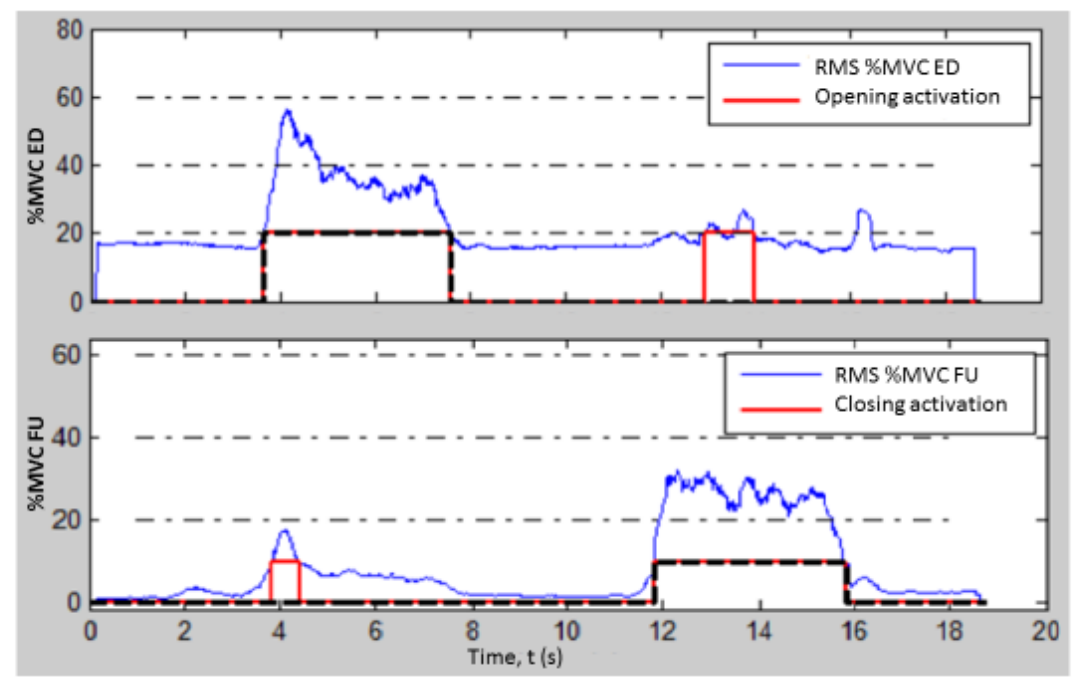

Fig. 10. Señales EMG normalizadas de los músculos FU y ED para determinar la apertura y cierre de la mano, mediante un control binario.

En la figura 10 se muestran las señales EMG normalizadas de los musculos ED y FU, y el resultado de aplicar un control binario con un umbral fijo del $20 \%$ para determinar si la mano está abierta o cerrada. La tasa de acierto es del 89.7 and $87.2 \%$ para cierre y apertura de mano, respectivamente. 


\section{Conclusiones}

El proyecto dentro del que se está desarrollando RobHand se encuentra en su tercer y último año de ejecución $\mathrm{y}$, en la actualidad, nos encontramos poniendo a punto los algoritmos de control háptico para soportar la realización de terapias activas asistidas basadas en la detección de la intención del paciente mediante el registro de sEMG. En este trabajo hemos presentado el planteamiento y el estado de desarrollo de la arquitectura de control del exoesqueleto de mano integrado en el sistema de rehabilitación. Se han descrito sus modelos cinemático y dinámico, y el controlador biocooperativo basado en sEMG. Una vez validada en laboratorio comenzarán las pruebas con pacientes en el Servicio de Rehabilitación del Hospital Clínico Universitario de Valladolid.

\section{Agradecimientos}

El trabajo reflejado en está comunicación está siendo realizado gracias a la financiación del Centro para el Desarrollo Tecnológico Industrial (CDTI, referencia IDI 2017 0263) y de la empresa CyL ImasD.

\section{Referencias}

CAMEIRAO, M. S. , BADIA, S. B., ZIMMERLI, L., OLLER, E. D. y VERSCHURE, P. F.(2007) "The Rehabilitation Gaming System: a Virtual Reality Based System for the Evaluation and Rehabilitation of Motor Deficits" en 2007 Virtual Rehabilitation, Venice, Italy: IEEE. 29-33

CISNAL, A., LOBO, V., MORENO, V., FRAILE, J. C. , ALONSO, R. y TURIEL, J.P. (2018) "Robhand, un exoesqueleto de mano para la rehabilitación neuromotora aplicando terapias activas y pasivas" en XXXIX Jornadas de Automática, Badajoz: CEA, vol. 2, 34-41

FLEISCHER, C., WEGE, A., KONDAK, K. \& HOMMEL, G., (2006) “Application of EMG signals for controlling exoskeleton robots” en Biomedizinische technik, vol. 51, no 5/6, p. 314-319

FRAILE, J. C., PÉREZ-TURIEL, J., BAEYENS, E., VIÑAS, P. et al (2016) "E2Rebot: a robotic platform for upper limb rehabilitation in patients with neuromotor disability" en Advances in Mechanical Engineering, 8(8), p. 1-13.

KURIKI., H. U., De AZEVEDO, F. M., OTA TAKASHASHI, L. S., MELLO, E. M., et al, (2013) "The Relationship Between Electromyography and Muscle Force", EMG Methods for Evaluation Muscle and Nerve Function, Intech, pp. 31-54.

LEHMAN, G. J. y MCGILL, S. M. (2013) “The Importance of Normalization in the Interpretation of Surface electromyography: A proof of Principle” en J. Manipulative Physiol. Ther., vol. 22, no. 7, p. 1-13. 
LUCAS, L., DICICCO, M. \& MATSUOKA, Y., (2011) “An EMG-driven Exoskeleton Hand Robotic Training Device on Chronic Stroke Subjects" en 2011 IEEE International Conference on Rehabilitation Robotics (ICORR). Zurich, Suiza: IEEE, 1-5.

PINHAS, B. T. y ZHOU, M. (2015) "Sensing and Force-Feedback Exoskeleton (SAFE) Robotic Glove” en IEEE Trans. Neural. Syst. Rehabil. Eng., vol. 23, no. 6, p. 992-1002.

SANDOVAL-GONZALEZ, O. O., JACINTO-VILLEGAS, J., HERRERA-AGUILAR, I., PORTILLO-RODIGUEZ, O. et al. (2016) "Design and Development of a Hand Exoskeleton Robot for Active and Passive Rehabilitation" en International Journal of Advanced Robotic Systems 13(66), p.1-12

UEKI, S., KAWASAKI, H., ITO, S., NISHIMOTO, Y. et al. (2012) "Development of a hand-assist robot with multi-degrees-of-freedom for rehabilitation therapy" en IEEE/ASME Trans. Mechatronics, vol. 17, no. 1, p. 136-146.

YAP, H. K., ANG, B. W., LIM, J. H., GOH, J. C., \& YEOW, C. H. (2016) “A Fabric-Regulated Soft Robotic Glove with User Intent Detection using EMG and RFID for Hand Assistive Application”, en 2016 IEEE International Conference on Robotics and Automation (ICRA), Estocolmo, Suecia: IEEE. $3537-42$ 


\title{
La estimulación mecánica aferente no reduce sistemáticamente el temblor de pacientes con temblor esencial
}

\author{
Lora-Millán, Julio Salvador ${ }^{a^{*}}$; López-Blanco, Roberto ${ }^{\mathrm{b}}$; Gallego, Juan Álvaro ${ }^{\mathrm{a}}$; \\ González de la Aleja, Jesús ${ }^{b}$; Rocon, Eduardo ${ }^{a}$ \\ ${ }^{a}$ Centro de automática y robótica (CAR), CSIC-UPM, Madrid, España, \\ 'Instituto de investigación sanitaria Hospital 12 de Octubre, Madrid, España. \\ *Autor al que dirigir correspondencia: julio.lora@csic.es
}

\begin{abstract}
In spite of being one of the major causes of disability, Essential Tremor (ET) is not effectively handled in 50\% of cases. We have hypothesised that tremor in ET could be reduced by recruiting afferent pathways through mechanical stimulation. We have designed a platform to stimulate the forearm and hand of the patient with piezoelectric actuators while the tremor is measured using inertial sensors. We have studied the effect of four different stimulation strategies in 18 ET patients, each stimulation strategy was characterized by the frequency of the vibration. Although the response to stimulation was very heterogeneous across patients and strategies, the majority of patients increased their tremor (between 50\% and 72\%, depending on the strategy), while a very reduced proportion (between 5 and 22\%) of patients decreased their tremor amplitude. However, we considered that these results were difficult to explain because the high variability of the tremor that we have assessed: during equally long trials without stimulation, tremor increased significantly in $45 \%$ of patients and decreased in $22 \%$ of them. Therefore, we claim that mechanical vibration does not have a consistent and homogenous effect on ET tremor.
\end{abstract}

Keywords: Essential tremor, mechanical stimulation, piezoelectric actuators, inertial sensors.

\footnotetext{
Resumen

A pesar de ser una de las principales causas de discapacidad, el temblor esencial (TE) no se consigue tratar de manera efectiva en el $50 \%$ de los casos diagnosticados. Nosotros partimos de la hipótesis de que, al estimular las vías aferentes a través de vibración mecánica, el temblor en pacientes con TE podría reducirse. Para comprobarlo, se ha desarrollado una plataforma para estimular el antebrazo y la mano del paciente usando actuadores piezoeléctricos mientras se mide el temblor utilizando sensores inerciales.
} 
La estimulación mecánica aferente no reduce sistemáticamente el temblor de pacientes con temblor esencial

Después de realizar ensayos con 18 sujetos, se ha analizado el efecto de aplicar cuatro estrategias de estimulación a diferente frecuencia de vibración. Aunque la respuesta a la estimulación fue muy heterogénea teniendo en cuenta los pacientes y las estrategias aplicadas, en la mayoría de los pacientes el temblor aumentó significativamente (entre 50\% y 72\%, según la estrategia), mientras que para una proporción reducida de pacientes (entre 5 y 22\%) disminuyó la amplitud de su temblor. No obstante, se considera que estos resultados son dificiles de explicar debido a la gran variabilidad del temblor: durante ensayos de igual duración, pero sin aplicar estimulación, el temblor aumentó significativamente en el $45 \%$ de los pacientes y disminuyó en el $22 \%$ de ellos. Por lo tanto, podemos afirmar que la vibración mecánica no tiene un efecto consistente y homogéneo en el temblor esencial.

Palabras clave: Temblor esencial, estimulación mecánica aferente, actuadores piezoeléctricos, sensores inerciales

\section{Introducción}

El temblor esencial (TE) es uno de los trastornos del movimiento más prevalentes en adultos afectando aproximadamente al 5\% de las personas mayores de 65 años (Louis 2005). El TE se manifiesta como un temblor postural o cinético bilateral, en gran parte simétrico, que afecta a las manos y los antebrazos, y suele ir acompañado de temblor en la cabeza (Deuschl 1998). Sin embargo, el temblor solo se controla de manera efectiva en el 50\% de todos los pacientes (Deuschl 2011), por lo que existe la necesidad de desarrollar nuevos tratamientos para el TE.

Estudios recientes indican que el temblor en el TE se origina debido a la proyección de oscilaciones patológicas en las vías cerebelo-tálamo-corticales hacia las motoneuronas que inervan los músculos afectados (Helmich 2013), aunque los mecanismos exactos siguen siendo desconocidos. Una hipótesis clásica propone que la oliva inferior es la causa última de temblor en el TE, debido a oscilaciones anormales en las vías olivo-cerebelosas que se transmiten a los circuitos tálamo-corticales (Deuschl 2000).

Los mecanorreceptores, incluidos los corpúsculos de Paccini y Meissner, son sensibles a los estímulos vibratorios. En animales anestesiados o descerebrados, los corpúsculos de Paccini responden a estímulos de alta frecuencia $(60-600 \mathrm{~Hz})$, mientras que los corpúsculos de Meissner responden a estímulos de frecuencia menor $(10-300 \mathrm{~Hz})$ (Mountcastle 1972). Las respuestas sensoriales de ambos tipos de receptores se proyectan al núcleo cuneiforme ipsilateral (Douglas 1978), el cual, tiene importantes proyecciones al tálamo y a la oliva 
inferior (Douglas 1978; Geborek 2012) y, por lo tanto, puede proporcionar una vía para modular los circuitos que median el temblor en el ET.

El temblor en el ET es tratado principalmente con fármacos o estimulación cerebral profunda, una técnica que requiere neurocirugía (Deuschl 2011). Actualmente, ciertos dispositivos portátiles no invasivos se postulan como alternativas a esta medicación, es el caso de sistemas de estimulación de vías aferentes (Dideriksen 2017; Dosen 2015) como el que nosotros planteamos, pero que, por el contrario, utilizan estimulación eléctrica. Aunque muchos de ellos mostraron mejoras claras durante los ensayos clínicos en la población analizada, ninguno de ellos, hasta donde sabemos, ha ido más allá de los ensayos de laboratorio.

En este documento se pretende analizar si los estímulos aferentes administrados a través de la vibración mecánica de la mano y el antebrazo podrían atenuar el temblor en TE. Nuestra hipótesis es que la vibración mecánica estimularía los corpúsculos de Paccini y, por lo tanto, modularía la actividad anormal en las vías relacionadas con el temblor, lo que a su vez reduciría su amplitud. Sin embargo, nuestros resultados no apoyan dicha hipótesis. Encontramos que en una muestra relativamente grande de pacientes $(n=18)$, la respuesta a la vibración fue en gran parte heterogénea, aumentando, disminuyendo o no afectando la amplitud del temblor, al variar los pacientes y las estrategias de estimulación. Además, también encontramos que durante el desarrollo de ensayos relativamente largos (4 min), la amplitud del temblor no es estable, incluso durante mediciones en ausencia de estimulación.

\section{Materiales y métodos}

\subsection{Plataforma}

Para la validación de la hipótesis antes planteada, se ha diseñado y construido una plataforma que suministra estímulos vibratorios, a diferentes frecuencias, en la mano y antebrazo del usuario, al mismo tiempo que se registra el movimiento de dorsoflexión de la muñeca. Los estímulos vibratorios se administran mediante actuadores piezoeléctricos (modelo QP-10W, para las yemas de los dedos; PPA-4011 para la mano; PPA-1022 para el antebrazo; todos de Mide Technology, EE. UU.), que se controlaron a $5 \mathrm{kHz}$ mediante controladores hápticos piezoeléctricos (DRV8662, Texas Instruments, EE. UU.) a través de una tarjeta de adquisición de datos (NI USB 6003, National Instrument, EE. UU.) conectada a un PC. La amplitud de la vibración mecánica se mantuvo constante durante el experimento y se ajustó a su valor máximo (correspondiendo a voltajes de $50 \mathrm{~V}$ para los PPA-1022, $75 \mathrm{~V}$ para los QP-10W y PPA-4011). El movimiento de la muñeca se monitorizó a $100 \mathrm{~Hz}$ con sensores inerciales (TechMCS, Technaid, SP). 
La estimulación mecánica aferente no reduce sistemáticamente el temblor de pacientes con temblor esencial

\subsection{Pacientes}

Pacientes con temblor esencial (TE) fueron reclutados por el departamento de Neurología del Hospital Universitario 12 de Octubre (Madrid, España). Se incluyeron pacientes mayores de edad que hubieran sido diagnosticados con TE. El comité ético del Hospital 12 de Octubre dio su aprobación al protocolo experimental, garantizando el cumplimiento de la Declaración de Helsinki.

El proceso de reclutamiento finalizó con un total de 18 pacientes aptos para participar en el ensayo, (seis mujeres, doce hombres; edad $75.8 \pm 7.9$ años, media $\pm \mathrm{SD}$; rango, 59-88). La duración promedio de la enfermedad fue de 13.6 \pm 11.2 años (rango 1-40 años). La gravedad del temblor varió de leve a grave, con una puntuación media de $2.1 \pm 0.9$ (rango, 1-4) de acuerdo con la escala de clasificación de temblor de Fahn-Tolosa-Marin.

\subsection{Protocolo experimental}

Los ensayos se llevaron a cabo en el brazo más afectado por el temblor. Durante los experimentos, los actuadores piezoeléctricos se ubicaron sobre las yemas de los dedos, la mano y el antebrazo, las áreas donde la densidad de corpúsculos de Paccini es mayor

a

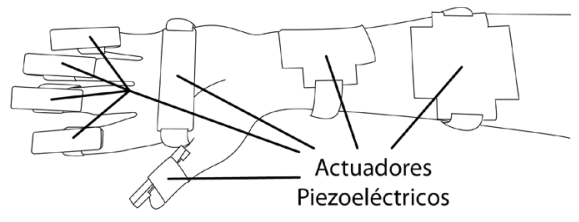

b

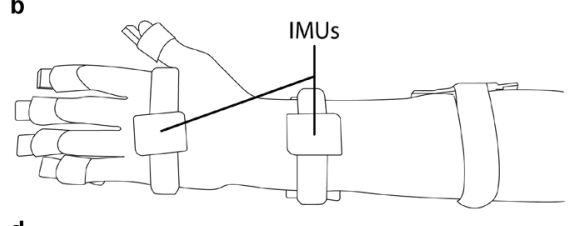

c

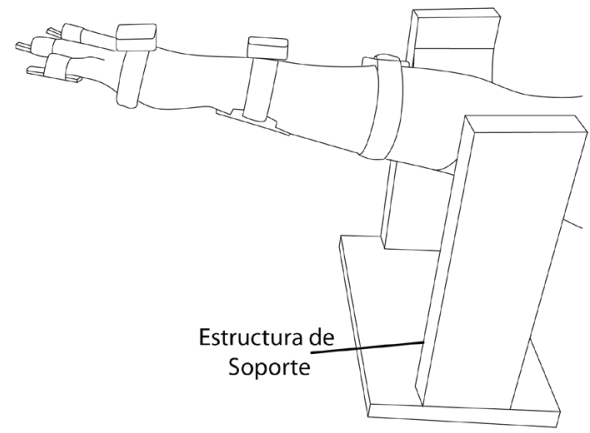

d

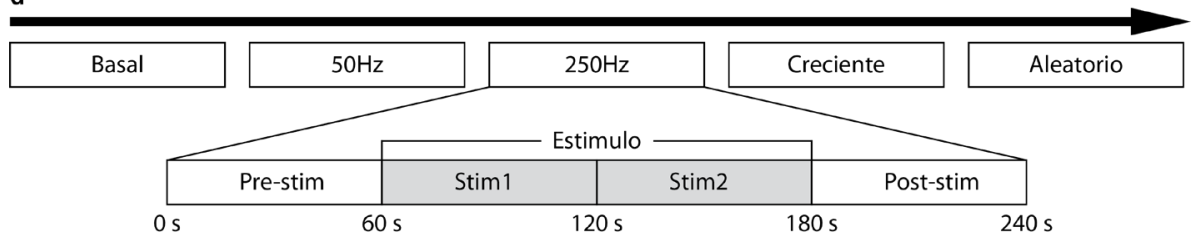

Figura 1. Desarrollo de la sesión y protocolo experimental. a. Localización de los actuadores piezoeléctricos utilizados para aplicar estímulos vibratorios (yemas de los dedos, manos y antebrazos; vista palmar). $b$.

Localización de las IMU utilizadas para registrar el temblor (vista dorsal). c. Estructura de soporte para realizar la tarea postural: los pacientes apoyaron la parte proximal de sus brazos en una banda elástica horizontal. $d$. Esquema del protocolo experimental. En la fila superior, cada rectángulo es una de las cinco estrategias de estimulación; El panel ampliado muestra cómo se dividió cada prueba en cuatro tramos de 60 s. 
(Roudaut 2012) (Figura 1a). Para medir el movimiento de la muñeca, colocamos sensores inerciales (Inertial Measurement Units, IMUs) en la parte dorsal de la mano y el antebrazo (Figura 1b). Los pacientes, sentados frente a un escritorio, realizaron una tarea postural estándar (Figura 1c), mientras que su brazo proximal descansaba sobre un soporte construido para tal propósito (nótese que el soporte no restringe los movimientos de la mano o el antebrazo). El soporte disminuye la fatiga muscular y asegura la repetibilidad en los ensayos. Durante las pruebas, los pacientes recibieron instrucciones de sostener el brazo, el antebrazo y la mano estirados contra la gravedad, para desencadenar el temblor.

El protocolo experimental consistió en cinco ensayos de 4 minutos en los que se aplicaron diferentes estrategias de estimulación (Figura 1d). Entre estos ensayos se intercalaron períodos de descanso de $10 \mathrm{~min}$. A continuación se detallan las citadas estrategias:

1. Sin estimulación ("Basal"): una medida de control en la que se grabó el temblor basal del paciente durante 240 segundos.

2. Estimulación a $50 \mathrm{~Hz}$ (“50Hz”): la vibración se administró a $50 \mathrm{~Hz}$, frecuencia que debería excitar mínimamente los corpúsculos de Paccini (Roudaut 2012).

3. Estimulación a $250 \mathrm{~Hz}$ ("250Hz"): la vibración se administró a $250 \mathrm{~Hz}$, frecuencia que debería excitar al máximo los corpúsculos de Paccini (Roudaut 2012).

4. Frecuencia de estimulación creciente ("Creciente"): la vibración se administró en intervalos de $50 \mathrm{~Hz}$ (orden ascendente, de $50 \mathrm{~Hz}$ a $450 \mathrm{~Hz}$ ); cada frecuencia se aplicó durante $13.33 \mathrm{~s}$.

5. Frecuencia de estimulación aleatoria ("Aleatorio"): la vibración se administró a las mismas frecuencias que durante los ensayos "Creciente", pero en orden aleatorio; Cada frecuencia se aplicó, de nuevo, durante 13.33 segundos.

Los ensayos 2 a 5 se dividieron en cuatro tramos de $60 \mathrm{~s}$ : durante el primer tramo (Pre-stim) evaluamos el temblor basal del paciente; durante los tramos 2 y 3 (Stim1 y Stim2), aplicamos estímulos vibratorios como se define en la estrategia correspondiente $(50 \mathrm{~Hz}, 250 \mathrm{~Hz}$, Creciente, Aleatorio); Durante el último tramo (Post-stim), evaluamos el temblor para detectar posibles efectos retardados.

\subsection{Análisis de los datos}

El temblor de los pacientes fue caracterizado por el movimiento de flexo-extensión de la muñeca, calculándose como la diferencia entre las velocidades angulares del antebrazo y de la mano medidas por los sensores inerciales. Dicha velocidad fue posteriormente filtrada utilizando un filtro paso-banda $\left(10^{\circ}\right.$ orden Butterworth, $\left.\mathrm{fc}=3-12 \mathrm{~Hz}\right)$. Por último, los datos son analizados mediante el valor cuadrático medio (root mean squeared, $R M S$ ) calculado en ventanas no superpuestas de 1 segundo. 
La estimulación mecánica aferente no reduce sistemáticamente el temblor de pacientes con temblor esencial

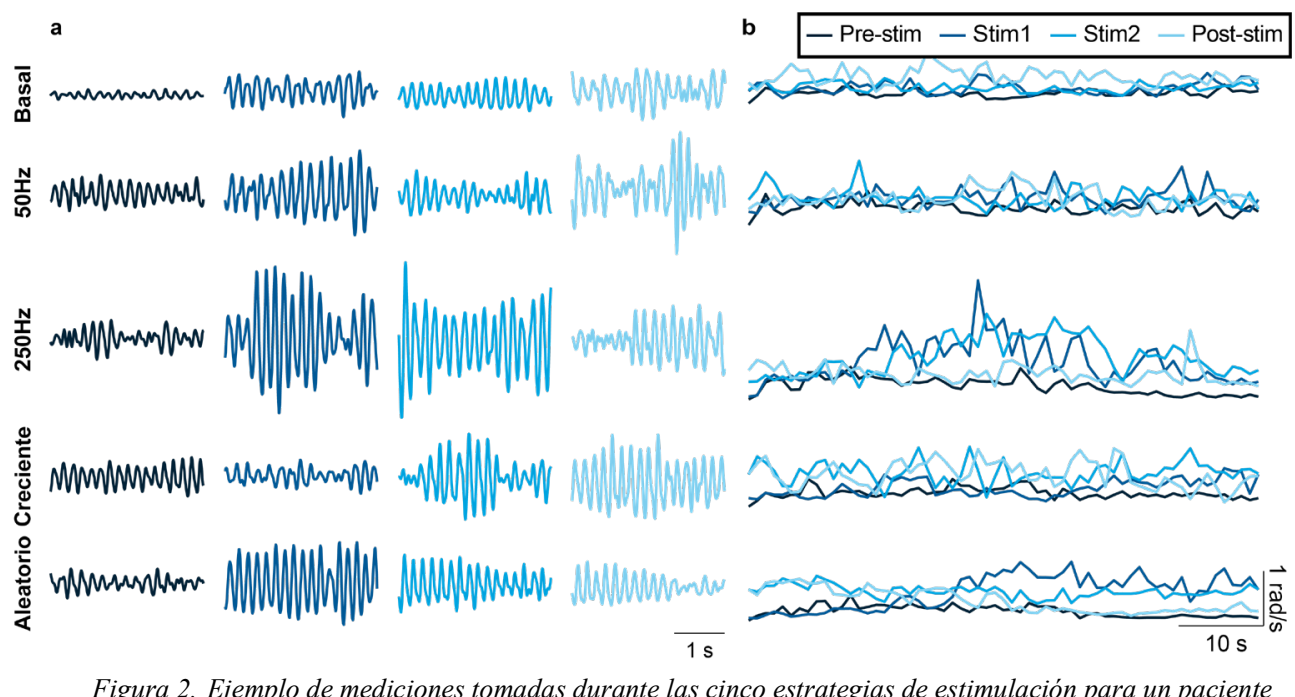

Figura 2. Ejemplo de mediciones tomadas durante las cinco estrategias de estimulación para un paciente (P4). Panel a. Amplitud del temblor (3s de datos) durante cada una de los cuatro tramos (Pre-stim, Stim1, Stim2, Post-stim; mostradas en diferentes columnas) para todas las estrategias de estimulación (agrupadas por fila);

Panel b. Amplitud del temblor durante cada una de las cinco estrategias en el mismo paciente. Cada trazo representa el valor RMS de la amplitud del temblor calculada en ventanas de 1 segundo.

\section{Resultados}

La Figura 2 muestra un ejemplo de los datos obtenidos durante las cinco estrategias de estimulación en un paciente. Como se puede ver en la fila superior de la Figura 2a, la amplitud del temblor varió con el tiempo incluso en la estrategia Basal. La Figura 2b muestra el procesamiento de los datos realizados: estimamos la amplitud del temblor en ventanas de $1 \mathrm{~s}$ no superpuestas para cada una de los tramos de 1 minuto (Pre-stim, Stim1, Stim2, Post-stim).

Nuestro objetivo es analizar la respuesta en cada paciente ante la estimulación, la Figura 3 muestra comparaciones detalladas entre los tramos PreStim y Stim (considerada como la unión de los tramos Stim1 y Stim2) para cada estrategia de estimulación en tres pacientes. En general, el efecto de cada estrategia de estimulación difiere entre ellos. Por ejemplo, durante la estimulación a $250 \mathrm{~Hz}$ : para el paciente P4, la amplitud del temblor aumentó considerablemente (Figura 3g); para el paciente P9, la amplitud del temblor disminuyó considerablemente (Figura 3i); y para el paciente P15, la amplitud del temblor apenas cambió (Figura 3j). Así mismo, incluso cuando no se produce estimulación, el comportamiento es diferente, aumentado, manteniéndose constante o disminuyendo el temblor conforme avanza el tiempo (Figuras 3a, 3b y 3c respectivamente). Esta variabilidad propia del temblor cuando no existe estimulación complica buscar relaciones de causa-efecto, pues no es posible saber si las variaciones que tienen lugar se deben al paso del tiempo o efectivamente son causadas 
por la estimulación. Por este motivo se ha descartado realizar ensayos utilizando los datos medidos después de la estimulación (tramo post-Stim).
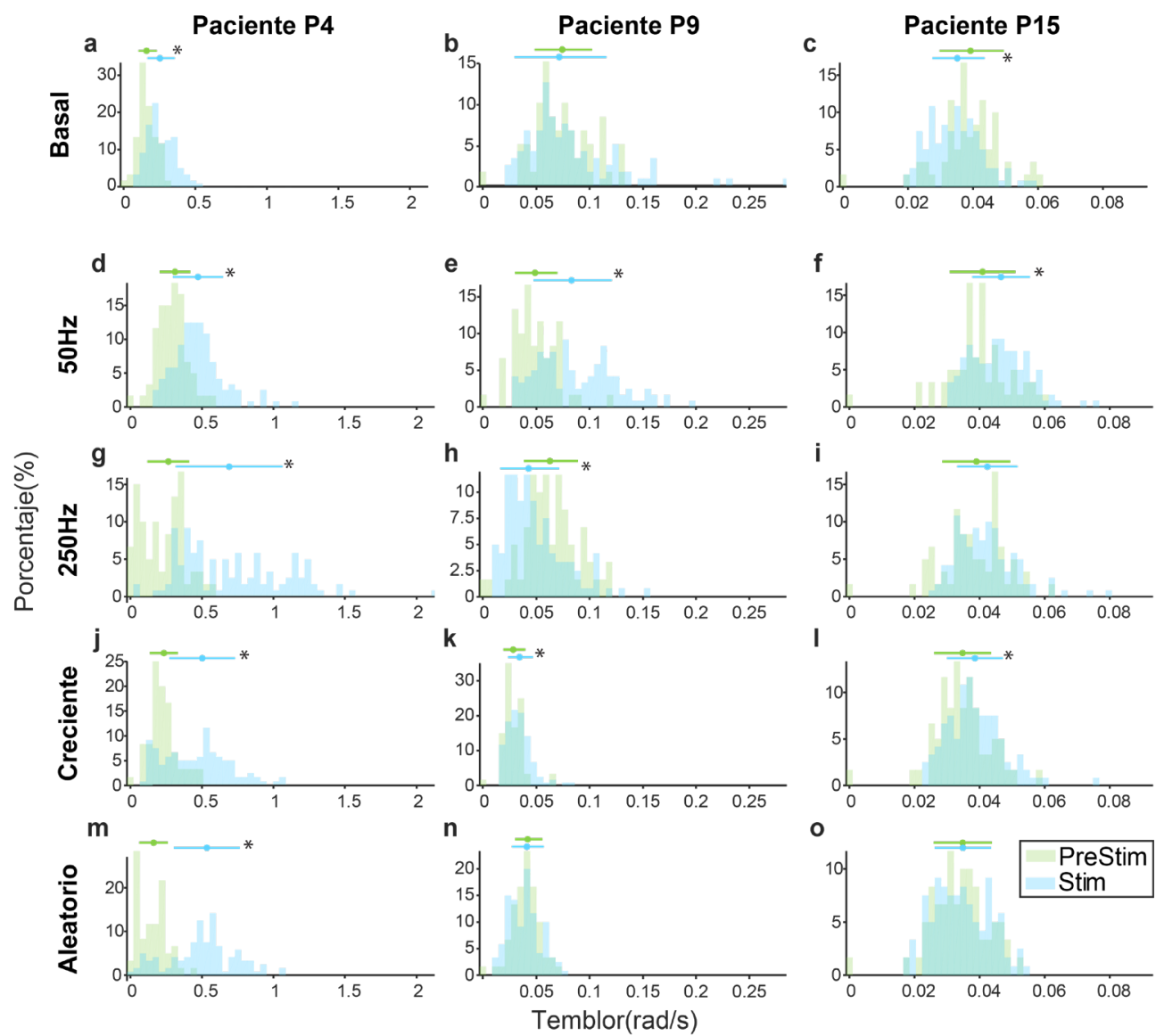

Figura 3. Ejemplos del efecto de las estrategias de estimulación en el temblor de tres pacientes (P4, P9 y P15). Cada panel compara el tramo Pre-stim (verde) con su correspondiente tramo de estimulación (Stim1 + Stim2; en azul). Cada histograma representa la distribución de la amplitud del temblor en intervalos de 1 s para la condición de estimulación correspondiente. Barras superiores: media $\pm S D$; * indica que los tramos Pre-Stim y Stim son significativamente diferentes $(P<0.01$, Test Mann-Witney).

La Figura 4 resume este análisis para los 18 pacientes. En general, todas las estrategias de estimulación aumentaron la amplitud del temblor en la mayoría de los casos, indicándose como la porción dominante en rojo en los resultados agrupados por estrategia estimulación (Figura 4b). Sin embargo, también hubo algunos pacientes para quienes el temblor se redujo o no se vio afectado (Figura 4a). Esta misma respuesta heterogénea se observó durante los ensayos NoStim. Además, incluso para los seis pacientes cuyo temblor se mantuvo sin 

esencial

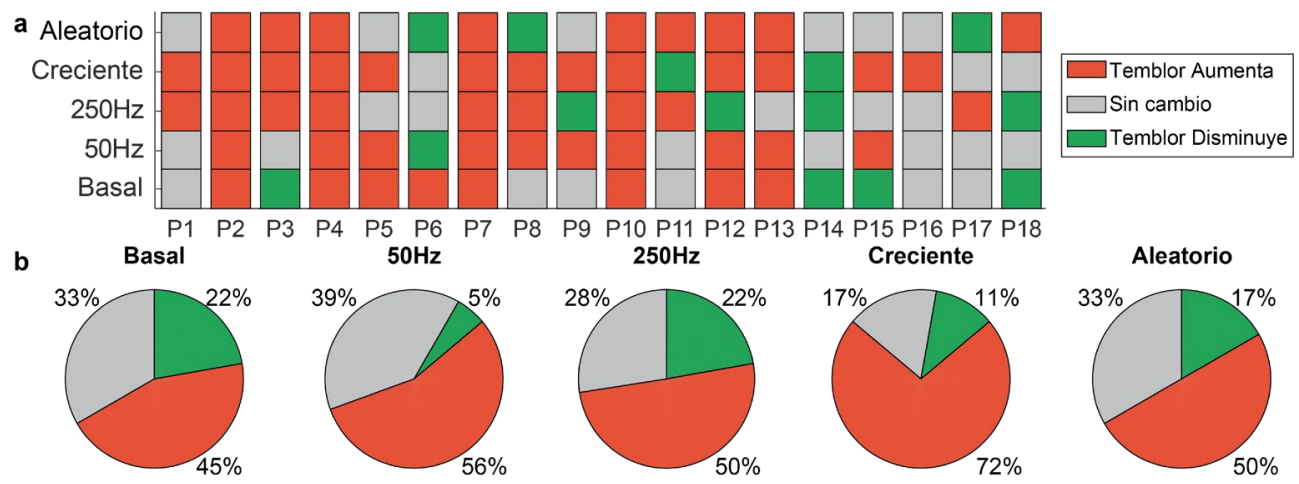

Figura 4. Resumen de la evolución en la amplitud del temblor durante las diferentes estrategias de estimulación. Panel a. Cambio en la amplitud del temblor durante cada tramo Stim con respecto al correspondiente tramo Pre-stim en cada paciente y cada estrategia de estimulación. Panel b. Porcentaje de pacientes para los cuales el temblor disminuyó, aumentó o permaneció inalterado.

cambios durante el ensayo NoStim, los cambios en la amplitud del temblor durante los ensayos de estimulación fueron muy heterogéneos, lo que resultó en un temblor que se incrementó principalmente ( $46 \%$; teniendo en cuenta todas las estrategias de estimulación en todos los pacientes), pero también disminuyeron (17\%) o no se vieron afectados $(37 \%)$.

Debido a la tendencia global, según la cual parece que el temblor aumentase al aplicar la estimulación, se ha intentado identificar un efecto de grupo, normalizando la amplitud del temblor dentro de cada paciente a su percentil 99 y agrupando los datos. El análisis de los resultados de estos grupos reveló un aumento significativo del temblor para todas las estrategias de estimulación ( $\mathrm{P} \sim 0$ para todas las comparaciones; Wilcoxon Rank Sum test; Figura 5), incluso en los ensayos NoStim.

\section{Discusión}

En este trabajo se ha analizado el efecto de la vibración mecánica del antebrazo y la mano sobre el temblor en TE. Nuestro objetivo era estimular selectivamente los corpúsculos de Paccini para interrumpir los mecanismos de generación del temblor en el cerebro, atenuándolo como consecuencia. Para ese fin, diseñamos un protocolo en el que suministramos estimulación vibratoria a diferente frecuencia y estudiamos su impacto en el temblor. En general, aunque la tendencia global parece ser que la vibración mecánica supone un aumento en la amplitud del temblor, la variabilidad entre pacientes fue excesiva como para sacar conclusiones significativas. 


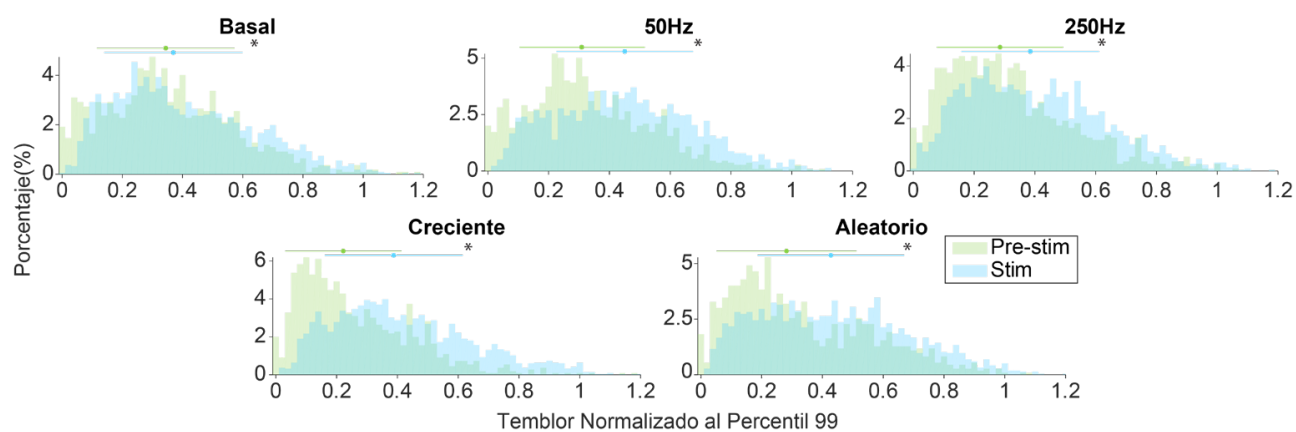

Figura 5. Análisis grupal de la evolución en la amplitud del temblor durante cada estrategia de estimulación. Cada panel compara la amplitud del temblor durante una estrategia de estimulación (azul) con su amplitud durante el tramo de pre-estimulación (verde). Para cada paciente y estrategia de estimulación, normalizamos la amplitud del temblor dividiéndolo por el percentil 99 de su distribución. Barras superiores: media $\pm S D$; * denota que los tramos Pre-Stim y Stim son significativamente diferentes $(P<0.01$, prueba de Wilcoxon Rank Sum test)

Hasta donde sabemos, ningún estudio previo había investigado el efecto de la vibración mecánica sobre el temblor en TE. Sin embargo, algunos grupos han usado la estimulación eléctrica sensorial (por debajo del umbral del motor) para tratar de reducir el temblor en el ET. En (Heo 2015), los autores demostraron que la estimulación eléctrica de los músculos principales del brazo y la muñeca a $100 \mathrm{~Hz}$ reduce la amplitud del temblor en TE. A pesar de que los resultados de su grupo fueron estadísticamente significativos, sus datos también parecen ser muy variables entre los pacientes y los ensayos, dada la amplitud de sus distribuciones de datos. Dideriksen et al. utilizaron recientemente la estimulación intramuscular y de superficie, también administrada a $100 \mathrm{~Hz}$, para estimular las fibras aferentes (entre otras vías) y reducir el temblor de cuatro pacientes con ET (Dideriksen 2017). Por lo tanto, la vibración mecánica parece afectar el temblor de una manera fundamentalmente diferente a la estimulación eléctrica aferente, pues la tendencia vista en nuestros ensayos es que el temblor se incrementaba.

\section{Conclusión}

En este documento se ha presenta el desarrollo de un sistema para reducir el temblor en el Temblor Esencial mediante la aplicación de estímulos vibratorios en el antebrazo y la mano. Nuestros resultados indican que la vibración mecánica estaba interrelacionada con cambios en gran parte heterogéneos en el temblor de los pacientes, aunque la tendencia dominante es a incrementar la amplitud del temblor. Cabe destacar que, durante nuestros ensayos 
La estimulación mecánica aferente no reduce sistemáticamente el temblor de pacientes con temblor esencial

relativamente largos, la variabilidad intrínseca del temblor, incluso sin estimulación, fue mayor de lo que esperábamos. Esta observación dificulta aún más la interpretación de nuestros datos, pero también sugiere que los nuevos protocolos experimentales deberían tener en cuenta la variabilidad intrínseca del temblor a la hora de analizar los resultados.

\section{Referencias}

Deuschl, Günther, Peter Bain, Mitchell Brin, Y Agid, L Benabid, R Benecke, A Berardelli, ET AL. 1998. "Consensus Statement of the Movement Disorder Society on Tremor.” Mov. Disord. 13 (S3): 2-23. https://doi.org/10.1002/mds.870131303.

Deuschl, GÜNTher, AND Rodger ElBle. 2000. "The Pathophysiology of Essential Tremor." Neurology $54 \quad$ (11 $\quad$ Suppl 4): http://www.ncbi.nlm.nih.gov/pubmed/10854347.

Deuschl, Günther, Jan Raethjen, Helge Hellriegel, AND Rodger Elble. 2011. Treatment of Patients with Essential Tremor. The Lancet Neurology. Vol. 10. https://doi.org/10.1016/S1474-4422(10)70322-7.

Dideriksen, Jakob L, Christopher M Laine, Strahinja Dosen, Silvia Muceli, Eduardo Rocon, José L Pons, Julian Benito-Leon, and Dario Farina. 2017. "Electrical Stimulation of Afferent Pathways for the Suppression of Pathological Tremor." Frontiers in Neuroscience 11 . https://doi.org/10.3389/fnins.2017.00178.

Dosen, Strahinja, Silvia Muceli, Jakob Lund Dideriksen, Juan Pablo Romero, EDUARDO ROCON, JoSE PONS, AND DARIO FARINA. 2015. “Online Tremor Suppression Using Electromyography and Low-Level Electrical Stimulation.” IEEE Transactions on Neural Systems and Rehabilitation Engineering 23 (3): 385-95. https://doi.org/10.1109/TNSRE.2014.2328296.

DOUGLAS, P R, D G FERringTON, AND M Rowe. 1978. "Coding of Information about Tactile Stimuli by Neurones of the Cuneate Nucleus." The Journal of Physiology 285 (1): 493513. https://doi.org/10.1113/jphysiol.1978.sp012585.

GEBOREK, PONTUS, HENRIK JÖRNTELl, AND FREDRIK BENGTSSON. 2012. "Stimulation within the Cuneate Nucleus Suppresses Synaptic Activation of Climbing Fibers." Frontiers in Neural Circuits 6 (January): 120. https://doi.org/10.3389/fncir.2012.00120.

Helmich, Rick C., IVAN TONi, GÜNTHER DEUSChl, AND BASTIAAN R. BloEM. 2013. "The Pathophysiology of Essential Tremor and Parkinson's Tremor." Current Neurology and Neuroscience Reports 13 (9): 378. https://doi.org/10.1007/s11910-013-0378-8.

Heo, Jae-Hoon Hoon, Ji-Won Won Kim, Yuri Kwon, SAnG-Ki Ki LeE, Gwang-Moon Moon Eom, Do-Young Young Kwon, Chan-Nyeong NyeOng LeE, Kun-Woo WOO PARK, AND MARIO MANTO. 2015. "Sensory Electrical Stimulation for Suppression of Postural Tremor in Patients with Essential Tremor." Bio-Medical Materials and Engineering 26: S803-9. https://doi.org/10.3233/BME-151372. 
LOUIS, ElAN D. 2005. "Essential Tremor." The Lancet Neurology 4 (2): 100-110. https://doi.org/10.1016/S1474-4422(05)00991-9.

MountCastle, V B, R H LAMotTe, AND G CARLI. 1972. "Detection Thresholds for Stimuli in Humans and Monkeys: Comparison with Threshold Events in Mechanoreceptive Afferent Nerve Fibers Innervating the Monkey Hand." Journal of Neurophysiology 35 (1): 122-36. https://doi.org/10.1152/jn.1972.35.1.122.

Roudaut, Yann, Aurélie Lonigro, Bertrand Coste, Jizhe HaO, PATRick Delmas, AND MARCEL CREST. 2012. "Touch Sense: Functional Organization and Molecular Determinants of Mechanosensitive Receptors." Channels (Austin, Tex.). Taylor \& Francis. https://doi.org/10.4161/chan.22213. 
USO DE EXOESQUELETOS

$11^{\circ} \mathrm{BE}$

EN EL ÁMBITO LABORAL 


\title{
Estimación de los esfuerzos generados en el hombro por parte de trabajadores de la industria del automóvil mediante modelos biomecánicos de medidas in situ
}

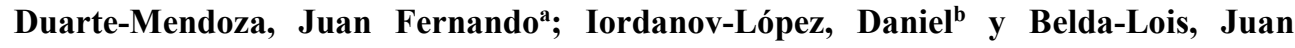 \\ Manuel $^{b}$ \\ ${ }^{a}$ Universitat Politècnica de València (UPV), Valencia, España. juaduame@etsii.upv.es, \\ ${ }^{\mathrm{b}}$ Instituto de Biomecánica (IBV), Universitat Politècnica de València (UPV), Valencia, España. \\ daniel.iordanov@,ibv.org,jmbelda@ibv.org
}

\begin{abstract}
Linear work in the automotive industry involves risks derived from repeated tasks and forced postures maintained over time. Although there are several platforms and tools that try to ergonomically evaluate jobs, such as the risk assessment software REBA and ERGO / IBV, these tools lack sensitivity when determining aspects related to the risk of injury. This happens especially when new aids are introduced in the workplace, different from the usual methods, which are not contemplated, such as the use of exoskeletons. That is why we present a methodology to estimate the muscular and joint efforts associated with linear work. We evaluate, ergo, 10 workers doing their usual work in real conditions. A superior limb model implemented in OpenSim is used, to which the dynamics of the movements measured with inertial sensors and the registration of the muscular signal of four muscles are introduced: anterior aspect of the deltoid, portion upper trapezius, the spinal erector and the large dorsal. The results obtained show a good consistency between the recorded muscle estimates and those estimated by the biomechanical model. These results can lay the practical basis for an objective assessment of risk in the workplace with novel conditions such as the use of exoskeletons.
\end{abstract}

Keywords: biomechanics models, effort estimation, exoskeletons.

\begin{abstract}
Resumen
Los trabajos lineales en la industria del automóvil involucran riesgos derivados de tareas repetidas y posturas forzadas mantenidas en el tiempo. Si bien, varias son las plataformas y herramientas que intentan evaluar ergonómicamente los puestos de trabajo, como los software de valoración de riesgos REBA y ERGO/IBV, estas herramientas carecen de sensibilidad a la hora de determinar aspectos relacionados con el riesgo de la lesión. Esto ocurre especialmente cuando se introducen nuevas ayudas en los puestos de
\end{abstract}


Estimación de esfuerzos generados en el hombro por parte de trabajadores de la industria del automóvil mediante modelos biomecánicos de medidas in situ

trabajo, distintas a los métodos habituales, que no están contempladas, como el uso de exoesqueletos. Es por ello que se presenta una metodología para estimar los esfuerzos musculares y articulares asociados al trabajo lineal. Evaluamos, ergo, a 10 trabajadores realizando su trabajo habitual en condiciones reales. Se utiliza un modelo de miembro superior implementado en OpenSim, al que se le introduce la dinámica de los movimientos medidos con sensores inerciales y con el registro de la señal muscular de cuatro músculos: cara anterior del deltoides, porción superior del trapecio, el erector espinal y el gran dorsal. Los resultados obtenidos muestran una buena consistencia entre las estimaciones musculares registradas y las estimadas por el modelo biomecánico. Estos resultados pueden sentar las bases prácticas para una valoración objetiva del riesgo en el puesto de trabajo con condiciones novedosas tal como el uso de exoesqueletos.

Palabras clave: modelos biomecánicos, estimación de esfuerzos, exoesqueletos.

\section{Introducción}

La industria del automóvil siempre ha sido uno de los sectores más importantes en el desarrollo y progreso de la economía de un país, no solo por su relevancia y aportación a la economía y al empleo, sino también por su capacidad de impulsar otros sectores. En España, supone ser un pilar estratégico, pues es uno de los principales propulsores de inversión y desarrollo en términos de innovación, seguridad y protección del medio ambiente, contribuyendo de manera significativa al desarrollo del país de forma directa, indirecta e incluso inducida. (Ramírez, 1993).

Su verdadera importancia radica, especialmente, en el hecho de que agrupa una serie de actividades de distinta naturaleza destacando las áreas encargadas de montaje de los productos fabricados. Es, justamente en estas áreas, donde se requieren esfuerzos mecánicos y movimientos forzosos por parte de los trabajadores para llevar a cabo las tareas requeridas. En muchas ocasiones dichas tareas con movimientos o posturas mantenidas en el tiempo superan los esfuerzos umbrales considerados normales, lo que puede conllevar la generación de trastornos musculo-esqueléticos en determinadas articulaciones. (Jonsson, 1982) La caracterización y comprensión de este tipo de tareas resulta esencial para mejorar las estrategias de ayuda enfocadas a la ergonomía y la reducción de riesgos en el trabajo. En España, este tipo de actividades que conllevan sobreesfuerzos suponen la afectación de más de 2500 personas de cada 100.000 trabajadores. (De Vicente, 2012). 
Para ello, hoy en día, se dispone de distintas herramientas que permiten obtener de alguna manera una primera valoración de los posibles esfuerzos que requiere un trabajo en concreto. Sin embargo, en la mayoría de ellas, lo que se evalúa simplemente es el análisis postural, en cuanto a aspectos ergonómicos se refiere, relacionado con el trabajo a realizar, dejando a un lado la valoración de un posible riesgo de lesión que éste pudiese generar. Estas herramientas suelen presentar una alta generalidad aplicable en muchas ocasiones, pero que implica que tengan una baja sensibilidad, es decir, los resultados obtenidos pueden ser pobres en detalles. Algunas de ellas, como el caso de los métodos REBA o RULA, han sido desarrolladas para la evaluación de las condiciones de trabajo (carga postural) estimando el riesgo de padecer desórdenes corporales en el trabajo (Hignett, 2000) o para investigar la exposición individual de los trabajadores a factores de riesgo de padecer transtornos musculoesqueléticos del miembro superior (Félix, 2011), respectivamente; o la aplicación ERGO/IBV - SOFTWARE EVALUACIÓN DE RIESGOS ERGONÓMICOS, diseñada para la evaluación y recomendación de diseño asociado a los riesgos ergonómicos y psicosociales de un puesto de trabajo. (ERGO/IBV - SOFTWARE EVALUACIÓN DE RIESGOS ERGONÓMICOS).

De igual manera sucede con la introducción de nuevos tipos de ayudas técnicas, novedosas con respecto a las técnicas clásicas, en gran parte debido al actual avance tecnológico, pero que no se encuentran contempladas en dichas herramientas de valoración funcional, como es el caso de los exoesqueletos.

Por otro lado, las técnicas de valoración que permiten obtener otras características de los movimientos realizados en las tareas del trabajo, como los ángulos relativos de los segmentos corporales, la cinemática de los mismos o incluso las fuerzas estimadas que llevan a cabo los músculos que forman parte de ellos, suponen ser herramientas que complementan a las de valoración funcional existentes, ayudando a potenciar la evaluación y determinación de los riesgos en el trabajo.

En este trabajo se describe una metología para la obtención de estimaciones de esfuerzos realizados en este tipo de trabajo por los grupos musculares implicados en la articulación del hombro o articulación glenohumeral: manguito de los rotadores (subescapular, infraespinoso, supraespinoso y redondo menor), trapecio y deltoides. Supone ser de las articulaciones más afectadas por el tipo de movimientos realizados, concretamente en aquellos en los que el brazo se encuentra por encima de la altura del hombro. Esto se debe a que se trata de una articulación holgada y poco profunda siendo la que mayor amplitud de movimientos permite, pero que a su vez es la que más luxaciones presenta. Anatómicamente, es una estructura articulada que sacrifica resistencia y estabilidad para obtener la gran amplitud de movimientos, necesarios para llevar a cabo diversas funciones. 
Estimación de esfuerzos generados en el hombro por parte de trabajadores de la industria del automóvil mediante modelos biomecánicos de medidas in situ

Se utilizan modelos biomecánicos que simulan la situación medida de la actuación del trabajador y se proporcionan las estimaciones de los esfuerzos que lleva consigo.

\section{Metodología}

Este trabajo propone un método para la estimación de esfuerzos realizados por los músculos implicados en la articulación del hombro. El primer paso consiste en la obtención de un modelo biomecánico adecuado del sujeto al que se desea evaluar en OPENSIM, con el que se puedan simular las medidas obtenidas para el mismo. Luego, se realiza un análisis cinemático inverso y un análisis dinámico inverso del modelo para un tiempo considerado en la toma de medidas. Por otra parte, se lleva a cabo un proceso de optimización estática con el que se obtendrán las estimaciones de los esfuerzos realizados por los distintos músculos.

\subsection{OpenSim: estimación de esfuerzos}

OPENSIM consiste en un software de código abierto que permite a los usuarios desarrollar modelos de estructuras musculoesqueléticas y crear simulaciones dinámicas de movimiento. (OPENSIM DOCUMENTATION) Con esta herramienta se consigue llevar a cabo la simulación y estimación deseada. De esta manera, se parte de un modelo de interés, concretamente aquel que simule de la forma más fidedigna posible y simplificada los movimientos de los miembros superiores, para así poder llevar a cabo un análisis de lo que sucede en la articulación del hombro. Se dispone de un conjunto de medidas de operarios en línea en condiciones reales que suponen ser la entrada del modelo.

\subsubsection{Escalado del modelo}

Se realiza el escalado del modelo para que presente propiedades semejantes y coincidentes con el sujeto de prueba, llevando a cabo una modificación de su antropometría. Para ello, OPENSIM ajusta las características de la masa en $\mathrm{kg}$ del sujeto y tiene en cuenta una serie de marcadores y segmentos de cuerpos rígidos definidos, 14 y 6 respectivamente, para el dimensionamiento del cuerpo. Se trata de un escalado basado en la medición, en la que se determinan los factores de escala para cada segmento mediante la comparación de puntos de referencia (marcadores virtuales y experimentales).

Se utiliza un conjunto marcadores y segmentos. Estos segmentos se corresponden con un cuerpo rígido del modelo y cuyos extremos están delimitados por los marcadores. Esto se puede apreciar en la Tabla 1. 


\subsubsection{Análisis cinemático inverso}

Con el modelo escalado se lleva a cabo un análisis cinemático empleando el toolbox de OPENSIM. Con este análisis, se consiguen los ángulos articulares del modelo musculoesquelético que mejor se ajustan a la cinemática experimental del sujeto. El problema de cinemática se resuelve para cada instante de tiempo (fotograma).

Tabla 1. Segmentos

\begin{tabular}{cccc}
\hline Nombre del segmento & Extremo distal & Extremo proximal & $\begin{array}{c}\text { Parte del modelo a la que se } \\
\text { asociada }\end{array}$ \\
\hline Brazo_d & pRightArmLatEpicondile & pRightAcromion & humerus \\
Brazo_i & pLeftArmLatEpicondile & pLeftAcromion & humerus_1 \\
Antebrazo_d & pRightUlnarStyloid & pRightArmMedEpicondile & ulna \\
Antebrazo_i & pLeftUlnarStyloid & pLeftArmMedEpicondile & ulna_1 \\
Hombro_d & pRightAcromion & pIJ & clavicle \\
Hombro_i & pLeftAcromion & pIJ & clavicle_1 \\
\hline
\end{tabular}

Con los resultados de la cinemática inversa resulta conveniente llevar a cabo una reducción de residuos, utilizando el algoritmo propio de OPENSIM para tal fin. Este algoritmo utiliza el modelo escalado y los resultados de la cinemática para realizar ajustes en las propiedades de inercia de los segmentos y la cinemática conjunta. Se obtiene, finalmente, un modelo y cinemática que son dinámicamente consistentes con las fuerzas que posteriormente se aplican.

\subsubsection{Análisis dinámico inverso}

Empleando el modelo escalado y los resultados de la cinemática se realiza un análisis dinámico del modelo empleando la correspondiente librería de OPENSIM, obteniendo la resolución de la estimación de fuerzas que afectan al modelo. Además, para este análisis resulta necesario añadir el valor de la fuerza que simula el peso de la herramienta que presenta el trabajador. Se crea de esta manera un fichero que presente dicho peso, una fuerza negativa en el eje y (según como se ha definido el modelo siguiendo los criterios de la ISB) con un valor de 2,5 $\mathrm{kg}$ que se corresponden con $24,5 \mathrm{~N}$, en una posición próxima a la mano con la que sujeta la herramienta. En este caso, se toma la situación de los marcadores de la mano derecha, pRightUlnarStyloid. 
Estimación de esfuerzos generados en el hombro por parte de trabajadores de la industria del automóvil mediante modelos biomecánicos de medidas in situ

Es necesario contar con los actuadores que participan en determinados movimientos (grados de libertad) del modelo y actúan como actuadores de reserva que conducen al modelo cuando no presenta determinados músculos o cuando la presencia de un conjunto de músculos es insuficiente para los requerimientos cinemáticos.

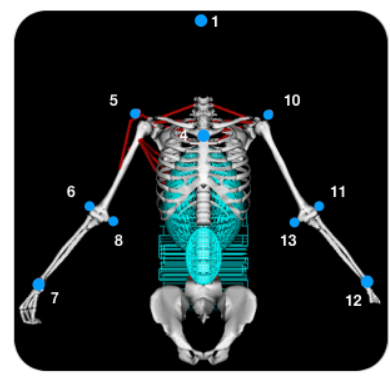

Vista anterior

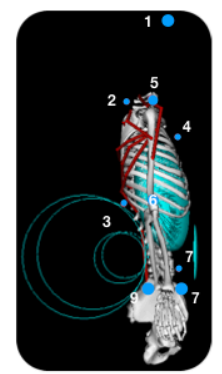

Vista lateral

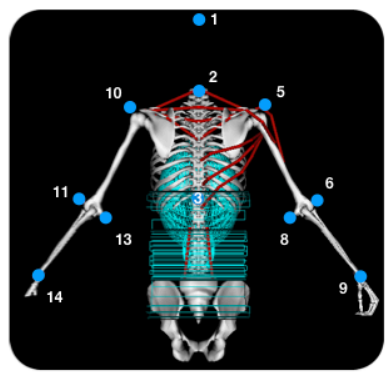

Vista posterior

\begin{tabular}{|c|c|c|}
\hline Marcadores derecha & Marcadores comunes & Marcadores izquierda \\
\hline 5. pRightAcromion & 1. pTopHead & 10. pLeftAcromion \\
\hline 6. pRightArmLatEpicondile & 2. pC7SpinalProcess & 11. pLeftArmLatEpicondile \\
\hline 7. pRightRadialStyloid & 3. pT12SpinalProcess & 12. pLeftRadialStyloid \\
\hline 8. pRightArmMedEpicondile & 4. pIJ & 13. pLeftArmMedEpicondile \\
\hline 9. pRightUlnarStyloid & - & 14. pLeftUlnarStyloid \\
\hline
\end{tabular}

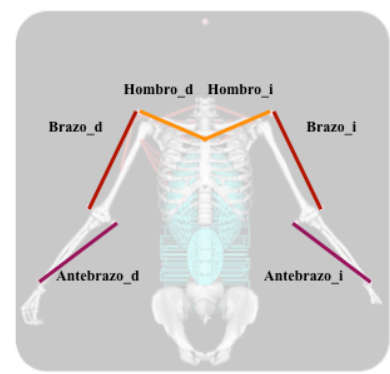

Fig. 1. Modelo "MoBL_ARMS_Upper_Limb": vista anterior, lateral y posterior, respectivamente. Marcadores. Segmentos definidos. Fuente: Creación propia (2019).

\subsubsection{Optimización estática}

Finalmente, la optimización estática se utiliza para abordar el problema de dinámica inversa resolviendo las ecuaciones de movimiento de las fuerzas generalizadas desconocidas que están sujetas a condiciones de activación de fuerza muscular y, así, poder estimar las activaciones y fuerzas musculares que las satisfacen.

Para una mayor simplificación del modelo, únicamente se consideran algunos músculos para el miembro superior derecho, entre los que se incluyen los involucrados en su articulación, y permitiendo los tres desplazamientos de la biomecánica del hombro: "elv_angle", "shoulder_elv" y "shoulder_rot", que se corresponden con los movimientos de aducción, flexo-extensión y rotación, respectivamente. 


\subsection{Modelo con muelle}

Toda la metodología anterior se aplica nuevamente sobre el mismo modelo, pero con una modificación de éste. En este caso, se introduce un resorte torsional en la articulación del hombro. Esta tipo de elemento de fuerza permite simular un resorte y un amortiguador sobre el rango de movimiento de una coordenada concreta, 'shoulder_elv', que se corresponde con el movimiento de flexión-extensión. Se simula un tope cuando la coordenada alcanza y supera su límite predefinido, es decir, su ángulo particular. El resorte añadido al modelo cuenta con las siguientes propiedades:

- Upper_stiffness: $-0.3 \mathrm{Nm} /$ grados

- Upper_limit: 5 grados

- Lower_stiffness: $1 \mathrm{Nm} /$ grados

- Lower_limit: -90 grados

- Damping: 0.00

- Transition: 2 grados

Donde estas características del elemento permiten la generación de fuerzas cuando el ángulo es mayor de $5^{\circ}$, con una activación de rigidez de $30 \mathrm{Nm} /$ grado, y una transición de 2 grados, cuando pasa de cero a $\mathrm{K}$ en el momento que la coordenada excede su límite. No se considera ningún tipo de amortiguación por lo que el damping debe estar a cero.

Con la adición del resorte se consigue simular el efecto de ayuda al movimiento que realizaría un exoesqueleto, en caso de que el trabajor lo llevase puesto.

\subsection{Comparación de los esfuerzos musculares}

De cada sujeto analizado, se obtiene la activación muscular y la estimación de fuerza muscular realizada por los distintos conjuntos de músculos estudiados, tanto para el modelo genérico como para el modelo que simula la existencia del exoesqueleto. En este paso, se pretende determinar si existen diferencias entre la presencia o ausencia del exoesqueleto, para un sujeto concreto en el mismo intervalo de tiempo, en algunas líneas de acción musculares consideradas relevantes por la posición del brazo en el movimiento analizado: TRAP1 (Trapecio), DELT2 y DELT3 (deltodides). Para ello, se distingue visualmente con las gráficas obtenidas para las fuerzas y se calculan los percentiles p25, p50 y p75, comparándolos entre ellos. 


\section{Materiales}

\subsection{Sujetos}

El planteamiento propuesto se aplicó sobre un total de 5 sujetos con exoesqueleto de un conjunto medidas in situ realizadas a un grupo de 12 trabajadores de la empresa de automóviles Ford Almussafes (Valencia), llevadas a cabo por el Instuto de Biomécanica de Valencia (IBV), de los cuales 6 trabajadores fueron considerados como casos y los otros 6 como controles, en función de si llevaban o no el exoesqueleto utilizado en las medidas, respectivamente. Todos los sujetos son varones sanos diestros, excepto uno de ellos que es una mujer, con alturas comprendidas entre 1,67 y 1,85 metros y pesos entre 64,4 y 83,3 kilogramos.

Para la obtención de las medidas se instrumentó a los sujetos con un sistema de EMG (electromiograma Ultium ${ }^{\mathrm{TM}}$ EMG) y un sistema de captura de movimiento (Xsens con el software MVN Analyze) basado en sensores inerciales, modelos biomecánicos y algoritmos de fusión de sensores. (XSENS MVN ANALYZE) Además, se les pidió a los trabajadores que llevaran a cabo dos test: uno de 3 ciclos del trabajo lineal realizado, que serviría como línea base (*para los casos considerados el test 1 es realizado sin exoesqueleto), y otro de 5 ciclos del trabajo lineal.

Tabla 2. Conjunto de medidas realizado

\begin{tabular}{c|cc}
\hline $\begin{array}{c}\text { Características de } \\
\text { la medida }\end{array}$ & $\begin{array}{c}\text { Controles (sin } \\
\text { exoesquelo) }\end{array}$ & $\begin{array}{c}\text { Casos (con } \\
\text { exoesqueleto)* }\end{array}$ \\
\hline $\mathrm{N}^{\mathrm{o}}$ sujetos & 6 & 6 \\
Test 1 & $\begin{array}{c}3 \text { ciclos de trabajo } \\
\text { (línea base) }\end{array}$ & $\begin{array}{c}3 \text { ciclos de trabajo (línea } \\
\text { base, sin exoesqueleto) }\end{array}$ \\
Test 2 & 5 ciclos de trabajo & $\begin{array}{c}5 \text { ciclos de trabajo (con } \\
\text { exoesqueleto) }\end{array}$ \\
\hline $\mathrm{N}^{\mathrm{o}}$ Medidas & 12 & 24 (2 días) \\
\hline
\end{tabular}

De las medidas realizadas se dispone directamente de los archivos ".tre" de las mismas en los que se encuentran las posiciones de todos los sensores a lo largo del tiempo. Se lleva a cabo, de esta manera, todo el proceso en 5 de los trabajadores casos del Test 2, que han realizado los 5 ciclos de trabajo con exoesqueleto. 


\subsection{Modelo Biomecánico}

El modelo biomecánico utilizado en OPENSIM, es un modelo simplificado del modelo original de "MoBL_ARMS_Upper_Limb_Model_OpenSim.osim", en el que se mantiene y destacan las componentes del tronco y miembros superiores, de acuerdo al objetivo del trabajo. Asimismo, el modelo modificado presenta los marcadores necesarios y rotulados de manera correcta, así como los músculos involucrados en el movimiento: dorsal latera, trapecio y deltoides. Cada músculo está representado por un múltiples líneas de acción dado que se trata de músculos extensos que tienen insersiones en extensiones grandes. Así:

- Dorsal lateral: LAT1, LAT2 y LAT3.

- Trapecio: TRAP1, TRAP2, TRAP3 y TRAP4.

- Deltoides: DELT2 y DELT3.

De la misma manera, se dispone del modelo “MoBL_ARMS_Upper_Limb_Model_OpenSim.osim" modificado, con la adición del muelle requerido para la simulación de portación del exoesqueleto.

\section{Resultados}

El tiempo de duración para cada una de las medidas de las que se dispone es alrededor de 5 minutos, donde se recoge todo el ciclo de trabajo: momentos en los que el brazo se sitúa por encima del hombro o alcanza su altura y el sujeto hace su actividad con la herramienta, así como aquellos en los que el sujeto simplemente está cogiendo o dejando la herramienta. Dado que nos interesa conocer especialmente los momentos en los que se sitúa el brazo por encima de la altura del hombro, con ayuda del movimiento simulado, seleccionamos y nos centramos en aquellos instantes de tiempo que presentan esa información de interés. Estos tramos considerados suelen tener una duración aproximada de 10 segundos.

El escalado se realiza para el modelo completo, al igual que la cinemática, incluyendo ambos miembros superiores. Sin embargo, dado que todos los trabajadores analizados son diestros, la dinámica se lleva a cado teniendo en cuenta solamente los músculos de la zona derecha. Por su parte, la optimización estática, se realiza bloqueando todos los grados de libertad del modelo y dejando únicamente los tres movimientos del hombro nombrados anteriormente.

Las estimaciones obtenidas para el brazo derecho de los distintos sujetos analizados con el modelo sin el exoesqueleto y con exoesqueleto simulado se pueden apreciar en la Figura 2. 
Estimación de esfuerzos generados en el hombro por parte de trabajadores de la industria del automóvil mediante modelos biomecánicos de medidas in situ
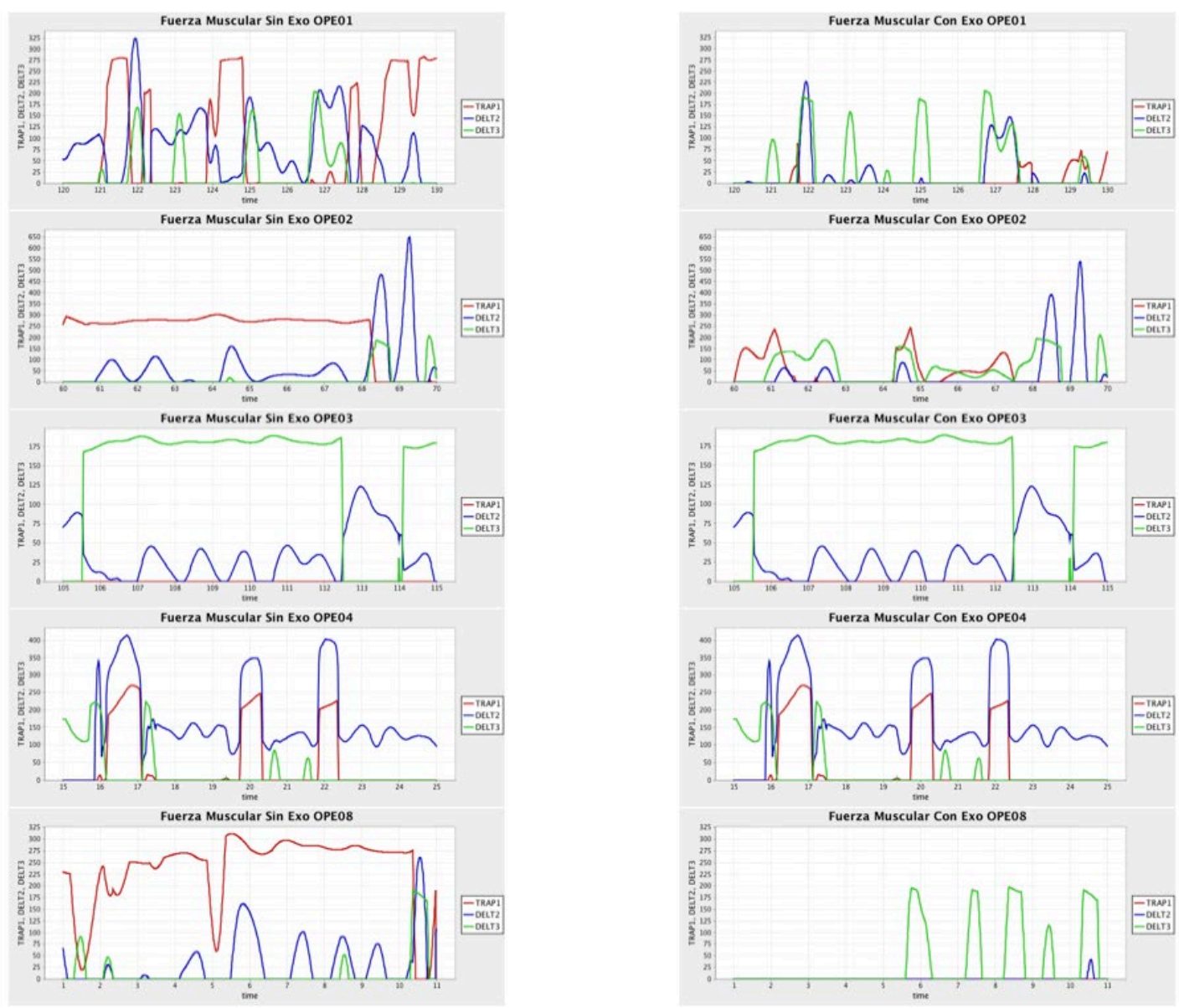

Fig. 2. Estimaciones musculares para los diferentes sujetos analizados: a la izqueirda se encuentran los sujetos con el modelo sin exoesqueleto y a la derecha con el modelo con exoesqueleto (resorte).

Fuente: Creación propia (2019).

Se aprecia que en algunos sujetos la presencia del exoesqueleto ayuda a una disminución de los esfuerzos realizados, especialmente dicha reducción es signifitiva en la línea de acción TRAP1 del músculo trapecio, el cual en ocasiones parece descender su esfuerzo en un 90 $95 \%$, tomando valores cercanos al cero, como en el caso del sujeto OPE01. En este caso, los esfuerzos de los músculos TRAP1 y DELT2 se ven atenuados significativamente, mientras que el DELT3 parece tomar los mismos valores e incluso valores ligeramente más elevados. En la mayoría de casos parece observarse que cuando los sujetos no presentan exoesqueleto los valores de esfuerzo en el DELT2 son mucho mayores que para el DELT3, 
pero una vez se tienen en cuenta el exoesqueleto la situación se invierte y es el DELT3 el que pasa a tener valores del mismo orden o incluso mucho mayores.

De la misma manera, sucede con el sujeto OPE02 y OPE08, en el que nuevamente los TRAP1 y DELT2 disminuyen, pero el DELT3 aumenta, aunque en estos casos a valores mucho más elevados que los que presentaban inicialmente. En OPE08 el TRAP1 parece incluso dejar de realizar un esfuerzo y tomar un valor constante de cero. Por otra parte, algunos sujetos como OPE03 y OPE04, la presencia del exoesqueleto no supone ninguna mejora en la reducción de los esfuerzos, siendo incluso idénticos tanto con él como sin él.

Finalmente, los percentiles p20, p50 y p75 obtenidos para los valores de los esfuerzos musculares de las líneas musculares TRAP1, DELT2 y DELT3 se recogen en la Tabla 3.

Tabla 3. Percentiles p20, p50 y p75 para diferentes líneas de acción musculares. Se resaltan en color rojo los aumentos en los valores de esfuerzos musculares.

\begin{tabular}{|c|c|c|c|c|c|c|c|c|c|c|}
\hline \multirow[b]{2}{*}{ Percentiles } & & \multicolumn{3}{|c|}{ TRAP1 } & \multicolumn{3}{|c|}{ DELT2 } & \multicolumn{3}{|c|}{ DELT3 } \\
\hline & & P25 & P50 & P75 & P25 & P50 & P75 & P25 & P50 & P75 \\
\hline \multirow[t]{2}{*}{ OPE01 } & Sin Exo & 0,001 & 0,040 & 214,549 & 14,207 & 81,117 & 116,967 & 0,000 & 0,000 & 2,337 \\
\hline & Con Exo & 0,000 & 0,000 & 0,007 & 0,000 & 0,000 & 5,841 & 0,000 & 0,000 & 53,683 \\
\hline \multirow[t]{2}{*}{ OPE02 } & Sin Exo & 264,148 & 275,974 & 278,974 & 0,127 & 29,417 & 76,314 & 0,000 & 0,000 & 0,000 \\
\hline & Con Exo & 0,000 & 0,004 & 65,881 & 0,000 & 0,000 & 5,321 & 0,000 & 44,599 & 120,148 \\
\hline \multirow[t]{2}{*}{ OPE03 } & Sin Exo & 0,000 & 0,000 & 0,000 & 7,550 & 26,502 & 43,643 & 172,768 & 180,055 & 182,222 \\
\hline & Con Exo & 0,000 & 0,000 & 0,000 & 7,550 & 26,502 & 43,643 & 172,768 & 180,055 & 182,222 \\
\hline \multirow[t]{2}{*}{ OPE04 } & Sin Exo & 0,000 & 0,000 & 4,014 & 111,188 & 130,947 & 158,363 & 0,000 & 0,000 & 0,000 \\
\hline & Con Exo & 0,000 & 0,000 & 4,014 & 111,188 & 130,947 & 158,363 & 0,000 & 0,000 & 0,000 \\
\hline \multirow[t]{2}{*}{ OPE05 } & Sin Exo & 0,001 & 226,936 & 273,209 & 0,000 & 0,001 & 23,133 & 0,000 & 0,000 & 0,000 \\
\hline & Con Exo & 0,000 & 0,000 & 0,000 & 0,000 & 0,000 & 0,000 & 0,000 & 0,000 & 10,579 \\
\hline
\end{tabular}

Con los percentiles se puede observar de forma cuantitativa, los mismos resultados obtenidos a partir de las gráficas. Para los operarios OPE01, OPE02 y OPE08, se identifican ciertas disminuciones cuando a los sujetos se les considera la colocación de un exoesqueleto, en las líneas musculares TRAP1 y DELT2; mientras que para el DELT3 se observa un aumento del esfuerzo realizado pasando de valores cercanos a cero a valores 
Estimación de esfuerzos generados en el hombro por parte de trabajadores de la industria del automóvil mediante modelos biomecánicos de medidas in situ

relativamente elevados: 53,683, 120,148 y 10,579, respectivamente. De igual manera, se comprueba que para los sujetos, OPE03 y OPE04, la colocación del exoesqueleto no supone ningún cambio en los valores de las estimaciones musculares.

\section{Discusión y conclusiones}

La análisis realizado con OPENSIM para los diferentes trabajadores considerados presenta discrepancias en los resultados obtenidos y puede que incluso contradictorios, pues en parte de ellos resulta que al comparar un movimiento, con el correspondiente esfuerzo que conlleva, con y sin un exoesqueleto que ayude a la realización de la tarea, efectivamente disminuyen los valores esfuerzo estimado en algunas de las líneas de acción que conforman los músculos principales del movimiento del hombro. Pero no afecta a todas ellas de la misma manera, ya que con el DELT3 pasa justo lo contrario y se ve aumentado. Mientras, en los otros, la presencia del exoesqueleto no supone cambios en los valores, no afecta el hecho de que un elemento de ayuda presente modifique el esfuerzo realizado por el trabajador.

Sin embargo, habría que tener algunas consideraciones en estos resultados para intentar explicar de alguna manera la diversidad que representan. Como se ha explicado en la metodología, los instantes de tiempo considerados son aquellos en los que los sujetos presentaban los brazos por encima de la línea del hombro y con la herramienta en la mano derecha. Dada esta posición es lógico, entonces, que el DELT2, por su localización anatómica anterior presente valores de activación mayores y mantenidos que en el DELT3, pues en este caso al tener los brazos ( $\sin$ ayuda de exoesqueleto) arriba supone un movimiento combinado de flexión-extensión y aducción-abducción, dado en parte por la contracción de la porción del deltoides anterior (DELT2), y por el TRAP1, que de igual forma colabora con la elevación del brazo incluso con una participación mayor. Por esta misma razón, cuando introducimos un elemento que simula el exoesqueleto y afectamos al movimiento "shoulder_elv", la contracción del mismo, así como la porción del trapecio, se ven disminuídas considerablemente, pues el resorte está ayudando al músculo a ejecer un esfuerzo de elevación del brazo y mantenerlo arriba. El efecto contrario sucede con el DELT3, el cual tira del brazo hacia abajo continuamente cuando se contrae y el hecho de que haya un elemento que lo eleva, provoca una mayor activación por su parte.

Ante esta situación, una posible manera de conseguir una disminución de todos los grupos musculares podría ser considerando otro tipo de elemento, y no simplemente un resorte, que a su vez ayude a la elevación de los brazos, y también permita una bajada amortiguada y suave, evitando que algunos músculos tengan que realizar un esfuerzo superior que en el que se realiza en una situación normal. 
En cuanto a los sujetos en los que el exoesqueleto no afecta al esfuerzo realizado, este hecho es debido a que, posiblemente, en los instantes considerados los movimientos realizados por los operarios no se llegue a entrar en el rango o a superar el límite con los que el resorte ha sido definido para actuar. De esta manera, al no verse dentro del rango de actuación determinado, el resorte no actúa y, por tanto, es como si el sujeto no llevase ningún elemento de ayuda y los valores de esfuerzos se mantienen iguales en ambos casos. Esto quiere decir, que aún con la presencia del exoesqueleto existen posturas en las que no parece aportar ningún tipo de ayuda a la realización de un trabajo concreto, generando situaciones en las que apoya y en las que no. Situaciones en las que también puede existir una postura o esfuerzo de riesgo para el paciente, y que el exoesqueleto no puede impedir, pues su actuación está limitada.

Finalmente, OPENSIM como una primera aproximación a una herramienta de evaluación de riesgos parece ser un buen método para llevar a cabo estimaciones de esfuerzos musculares, pues los resultados que se pueden extraer de él se acoplan de mejor o peor manera a la realidad, aunque se pueden explicar correctamente. No obstante, a la hora de realizar todo un análisis de estimaciones hay que tener en cuenta las limitaciones que puede presentar el programa y lo poco robusto que puede llegar a ser. Aún así, los resultados obtenidos, a pesar de las limitaciones y posibles simplificaciones en los modelos, son consistentes con los esfuerzos realizados realmente y pueden ayudar a determinar aquellas situaciones de riesgo para los trabajadores.

En estudios que requieran resultados más exactos y más realistas, sería conveniente no considerar simplificaciones, disponiendo de un modelo mucho más completo, con toda la anatomía involucrada en el movimiento total; realizar el estudio para muchos más instantes de tiempo, incluso que se estudie un ciclo de trabajo entero; y, disponer, de un elemento simulador de exoesqueleto con más variedad de características y restricciones que se pueden ajustar a una reproducción más fidedigna de un exoesqueleto real.

\section{Referencias}

DELP, S y col. (2007). "OpenSim: Open-Source Software to Create and Analyze Dynamic Simulations of Movement" en Biomedical Engineering, IEEE Transactions, vol. 54, p. 1940-50. DOI: 10.1109/TBME.2007.901024.

DE VICENTE, Á y col. (2012). "El transtorno Musculoesquelético en el ámbito laboral en cifras" en Departamento de Información e Investigación del Instituto Nacional de Seguridad e Higiene en el Trabajo, NIPO: 272-13-027-7.

FÉLIX, M. (2011). “Tareas repititivas II: Evaluación del riesgo para 1 aextremidad superior” en Instituo Nacional de Seguridad e Higiene en el Trabajo, Centro Nacional de Nuevas Tecnologías. 
Estimación de esfuerzos generados en el hombro por parte de trabajadores de la industria del automóvil mediante modelos biomecánicos de medidas in situ

HIGNETT, S y MCATAMNEY, L. (2000). "Rapid entire body assessnment (REBA)" en Applied Ergonomics, vol. 31, p. 201-5. DOI: 10.1016/S0003-6870(99)00039-3.

INSTITUTO DE BIOMECÁNICA. Ergo/IBV - Software Evaluación de Riesgos Ergonómicos. $<$ https://www.ibv.org/productos-y-servicios/productos/aplicaciones-tic/ergoibv-software-evaluacionde-riesgos-ergonomicos $>$ [Consulta: 23-Abril-2019]

JONSSON, B. (1982). "Measurement and evaluation of local muscular strain in the shoulder during constrained work" en Journal of human ergology, vol. 11, p. 73-88. DOI: 10.11183/jhe1972.11.73.

KAPANDJI, I. A. (1982). Cuadernos de fisiologí-a articular : esquemas comentados de mecánica articular. Cuaderno I, Miembro superior. El hombro. El codo. Pronosupinación. La muñeca. La mano y los dedos. Barcelona: Toray-Masson, Edi. 4. ISBN: 8431101598.

NCSRR (The National Center for Simulation in Rehanilitation Research). OpenSim Documentation. $<$ https://simtk-confluence.stanford.edu:8443/display/OpenSim/Documentation> [Consulta: 30-Abril2019]

RAMÍREZ MUÑOZ, R (1993). La industria de la automoción: su evolución e incidencia social y económica. Madrid: Editorial Complutense.

SAUL, K y col. (2014). "Benchmarking of dynamic simulation predictions in two software platforms using an upper limb musculoskeletal model" en Computer methods in biomechanics and biomedical engineering, vol. 18, p. 1-14. DOI: 10.1080/10255842.2014.916698.

WU, G y col. (2005). "ISB recommendation on definitions of joint coordinate systems of various joints for the reporting of human joint motion - Part II: Shoulder, elbow, wrist and hand" en Journal of biomechanics, vol. 38, p. 981-92. DOI: 10.1016/j.jbiomech.2004.05.042.

XSENS. Xsens MVN Analyze. <https://www.xsens.com/products/xsens-mvn-analyze/> [Consulta: 23Abril-2019]

ZHOU, H. y col. (2006). "Inertial measurements of upper limb motion" en Medical \& Biological Engineering \& Computing, vol. 44, p. 479-87. DOI: 10.1007/s11517-006-0063-z. 


\title{
Desarrollo de un modelo de exoesqueleto de hombro mediante OpenSim ${ }^{\circledR}$
}

\author{
Elguea-Aguinaco, Íñigo ${ }^{a}$; Belda-Lois, Juan Manuel $^{\text {b }}$ \\ ${ }^{a}$ Universidad Politécnica de Valencia, España. E-mail: iielag@etsii.upv.es

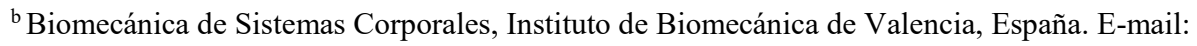 \\ jmbelda@ibv.org
}

\begin{abstract}
The fourth industrial revolution faces the technological challenge of humanrobot cooperation in manufacturing processes. Exoskeletons are an example of human-robot collaboration in which the device is worn by the human and the physical contact between the operator and the mechanical structure enhances individual's physical abilities. Currently, the first evaluations of the efficiency of this type of aid are being carried out, although these are fundamentally comparative in terms of muscular effort measured with the use and without the use of the exoskeleton. This project presents a passive upper limb exoskeleton model developed using OpenSim ${ }^{\circledR}$ free software, which allows estimating muscle and joint efforts for different tasks, under different conditions. The model has been validated with workers registered in real conditions, about who have records of their muscular activity perfoming the same tasks with and without exoskeleton. The results show an agreement on muscle activity with and without the device in the recorded muscles, which allows the model to be validated for the analysis of generic workplaces in which this type of aid is intended to be implemented.
\end{abstract}

Keywords: exoskeleton, passive, upper limb, muscular, joint

\begin{abstract}
Resumen
La cuarta revolución industrial se enfrenta al reto tecnológico de la cooperación entre el hombre y el robot en los procesos de fabricación. Los exoesqueletos son un ejemplo de esta colaboración humano-robot, en la que el dispositivo es vestido por el humano y el simple contacto físico entre el operador y la estructura mecánica permite el aumento de las capacidades físicas del individuo. Actualmente, se están llevando a cabo las primeras valoraciones de la eficiencia de este tipo de ayudas, si bien éstas son fundamentalmente comparativas en cuanto al esfuerzo muscular medido con el uso y sin el uso del exoesqueleto. Este trabajo presenta un modelo de exoesqueleto pasivo de miembro superior desarrollado mediante el software
\end{abstract}


libre OpenSim $\AA$, el cual permite estimar los esfuerzos musculares $y$ articulares para distintas tareas y distintas condiciones. El modelo se ha validado con trabajadores registrados en condiciones reales, de los que se disponen registros de su actividad muscular realizando las mismas tareas con exoesqueleto y sin él. Los resultados muestran un buen acuerdo en la actividad muscular con y sin el dispositivo en los músculos registrados, lo que permite validar el modelo para el análisis de puestos de trabajo genéricos en los que se pretenda implantar este tipo de ayudas.

Palabras clave: exoesqueleto, pasivo, miembro superior, muscular, articular.

\section{Introducción}

Debido a la creciente competencia internacional en el sector industrial, las compañías se encuentran envueltas en una constante mejora de la precisión de sus dispositivos automatizados. Sin embargo, algunos puestos de trabajo, hoy en día, siguen requiriendo de operaciones complejas, agarres precisos y destreza, donde estos dispositivos presentan ciertas limitaciones correspondientes a la viabilidad, la percepción, la velocidad y/o la flexibilidad. Es por ello que ciertas de estas tareas son realizadas manualmente por operarios. Dichas tareas suelen corresponderse con contracciones musculares isométricas y movimientos repetitivos de escasa duración, donde las manos se sitúan a la altura o por encima del acromion humeral. Éstas actividades suelen provocar trastornos musculo-esqueléticos en un alto porcentaje de los trabajadores (Otten et al., 2018; Spada et al., 2017; Sylla et al., 2014).

Los trastornos musculo-esqueléticos son un conjunto de lesiones inflamatorias y/o degenerativas que afectan principalmente a los hueso, músculos, ligamentos, tendones y nervios, y corresponden la segunda causa de discapaciodad en la población mundial.

En Estados Unidos, los trastornos musculo-esqueléticos de hombro resultan en una media de 23 días de baja laboral, mientras que otro tipo de lesiones del miembro superior únicamente implican de 7 a 10 días (Kim et al., 2018). En España, en la industria automovilística, por cada 100.000 trabajadores, 200`1 sufren algún tipo de lesión por sobreesfuerzos en esta área del cuerpo (Departamento de Información e Investigación del Instituto Nacional de Seguridad e Higiene en el Trabajo, 2012). Considerando dichas situaciones, se sugiere la necesidad de nuevas soluciones con el fin de favorecer y disminuir el trabajo físico de los operarios.

Un exoesqueleto puede definirse como un dispositivo activo o pasivo que se adapta al cuerpo del portador con el objeto de facilitar un movimiento tanto en esfuerzo como en trayectoria. Es por ello que el principal beneficio de un exoesqueleto se basa en la combinación entre la 
flexibilidad humana y la mejora de la potencia a través de la estructura mecánica, sin necesidad de enseñanza o programación robótica (Sylla et al., 2014).

Sin embargo, muchos de los exoesqueletos que están siendo desarrollados en la actualidad, no están diseñados considerando la complejidad del sistema musculo-esquelético, y restringen ciertos movimientos naturales durante el desempeño de las tareas, al limitar los grados de libertad de los miembros superiores.

Asimismo, la mayoría de los estudios llevados a cabo con este tipo de estructuras mecánicas se realizan a través de tareas simuladas en laboratorios, dónde no se conoce con exactitud la carga biomecánica de trabajo que será asistida por el exoesqueleto y las repercusiones potenciales que causará en los métodos de trabajo. Es por ello que, la introducción de este tipo de dispositivos en un ambiente de trabajo habitual puede revelar obstáculos en la aceptación del trabajador respecto a los exoesqueletos que no son evidentes en un ambiente de laboratorio controlado.

Por ello, se han realizado mediciones de los movimientos llevados a cabo por los operarios en su ambiente de trabajo a través de sensores inerciales. Asimismo, se han obtenido registros de la actividad muscular de los trabajadores con el uso y sin el uso del exoesqueleto. De esta forma, se han podido verificar y validar los resultados obtenidos a través del modelo desarrollado en SolidWorks ${ }^{\circledR}$ y $\operatorname{OpenSim}{ }^{\circledR}$, con los valores registrados en la actividad muscular de los trabajadores en los distintos músculos estudiados, durante la realización de las tareas con el empleo y sin el empleo de la estructura mecánica.

\section{Materiales y métodos}

\subsection{Programas informáticos}

\subsubsection{Xsens ${ }^{\circledR}$}

Xsens ${ }^{\circledR}$ es un software destinado al seguimiento y registro de movimiento en 3D. Para llevar a cabo dicha tarea, hace uso de una unidad de medición inercial magnética (IMMU), la cual comprende un acelerómetro $3 \mathrm{D}$, un magnetómetro $3 \mathrm{D}$ y un giroscopio $3 \mathrm{D}$ en un único dispositivo.

Para aquellos estudios ergonómicos dónde los cables pueden llegar a resultar un obstáculo, Xsens ${ }^{\circledR}$ posee el sistema MTw, un rastreador de movimiento incercial-magnético 
inalámbrico, desarrollado para una cinemática 3D ambulatoria de alta precisión. Este sistema ha sido empleado para realizar las mediciones de este trabajo.

Cada dispositivo de rastreo transmite los datos obtenidos de manera inalámbrica al ordenador a través de Awinda Master, un dongle USB conectado al PC. Comúnmente, este registro de datos no suele presentar la misma precisión que aquellos registros a través de un sistema de cableado. Sin embargo, Xsens ${ }^{\circledR}$ ha desarrollado un canal de procesado de señal que ha incorporado en el sistema de rastreo inalámbrico. Para ello, se ha creado un algoritmo que garantiza una alta precisión en condiciones dinámicas independiente de la velocidad de salida de los datos. Asimismo, se ha diseñado un protocolo de radio propio, llamado Awinda, para detectar y manejar pérdidas ocasionales de paquetes en el procesamiento en tiempo real. En caso de que los datos no se hayan transmitido adecuadamente, se almacenan en una memoria interna del rasteadror y se vuelven a transmitir cuando sea posible. Además, el protocolo Awinda es capaz de reducir la velocidad de salida de los datos, lo que, en combinación con el algoritmo, evita el deterioro de la precisión cuando se producen pérdidas de paquetes de datos. Awinda también resuelve el problema de la sincronización, proporcionando una sincronización precisa para hasta 20 MTws en $10 \mu \mathrm{s}$, lo que permite que el rendimiento del sistema sea similar al de un sistema cableado (Paulich et al., n.d.). La Figura 1 muestra los componentes de este sistema.
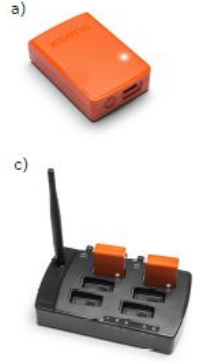

b)
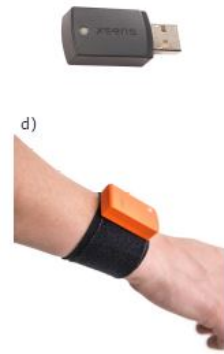

Fig. 1. Hardware Xsens MTw Awinda: a) rastreador de movimiento MTw; b) Awinda Dongle; c) estación Awinda; d) correa corporal MTw (Paulich et al., n.d.)

\subsubsection{SolidWorks ${ }^{\circledR}$}

SolidWorks ${ }^{\circledR}$ es un software de diseño CAD (diseño asistido por computadora) para modelar piezas y ensamblajes en 3D y planos en 2D. Ofrece un gran rango de soluciones correspondientes al proceso de desarrollo del producto. Permite crear, diseñar, simular, fabricar, publicar y gestionar los datos del proceso de diseño ("SolidWorks - Qué es y para qué sirve," n.d.). 


\subsubsection{OpenSim ${ }^{\circledR}$}

OpenSim ${ }^{\circledR}$ es una plataforma de código libre, desarrollado por el centro Computacional de Biomédica de la Universidad de Stanford, para modelar, simular y analizar el sistema neuromusculoesquelético. A diferencia de otros programas, OpenSim ${ }^{\circledR}$ permite que los investigadores y los laboratorios realicen mejoras en el software y que éstas puedan ser compartidas, con el fin de obtener un programa más dinámico. Por ello, se dispone de una página web (http://simtk.org/), que sirve como repositorio público de datos, modelos y herramientas computacionales relacionadas con la simulación que pueden ser compatidas con toda la comunidad que utilice el programa.

El software está escrito en ANSI C++, y la interfaz gráfica del usuario está escrita en Java (Delp et al., 2007). En la Figura 2, se muestra la interfaz de OpenSim ${ }^{\circledR}$ y el modelo utilizado para el estudio.

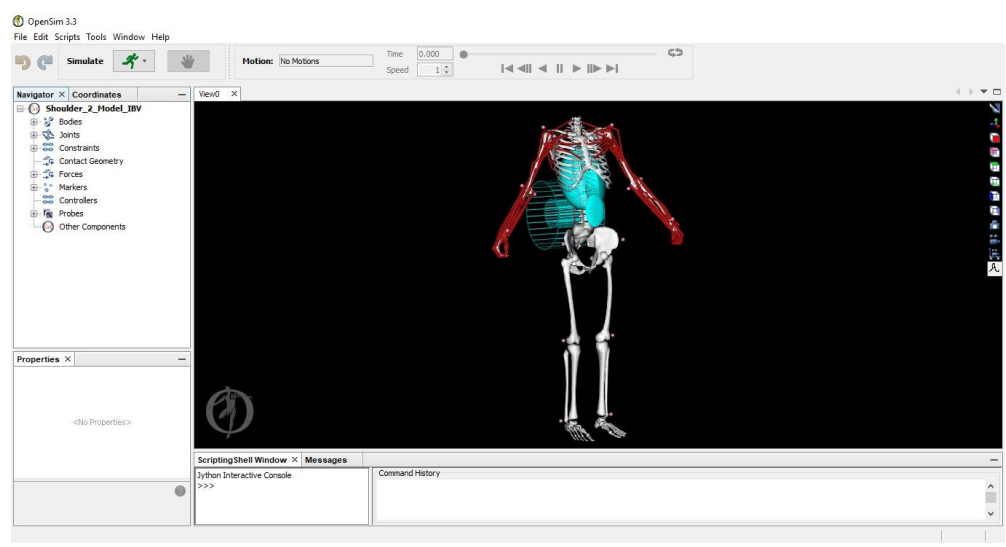

Fig. 2. Interfaz de OpenSim ${ }^{\circledR}$ (Fuente propia)

\subsection{Registro del movimiento}

El estudio se llevó a cabo en la planta de ensamblaje de automóviles de Ford Valencia Body and Assembly, situada en Almussafes. En él, partiparon 12 operarios (estatura: $1.75 \mathrm{~m} \pm 5$ cm, peso: $73.95 \mathrm{~kg} \pm 4.65 \mathrm{~kg}$, edad: 36 años \pm 5 años) a los que se les realizaron mediciones de los movimientos y regitros de las actividades musculares durante el desempeño de distintas tareas. Para ello, se hizo uso de la tecnología MTw Awinda de Xsens ${ }^{\circledR}$, a través de la cual se instrumentaron las extremidades superiores de cada operario mediante nueve sensores inerciales. Después de realizar las respectivas calibraciones (Figura 3a)) para cada sujeto, se midió a cada operario en distintas estaciones de atornillado de piezas por encima 
del acromion humeral en ejercicios de tres y cinco ciclos. Durante estas actividades, los trabajadores utilizaron una herramienta de aproximadamente $24.5 \mathrm{~N}$ de peso. Estas mediciones se realizaron sin el uso (Figura 3b)) y con el uso (Figura 3c)) del exoesqueleto.

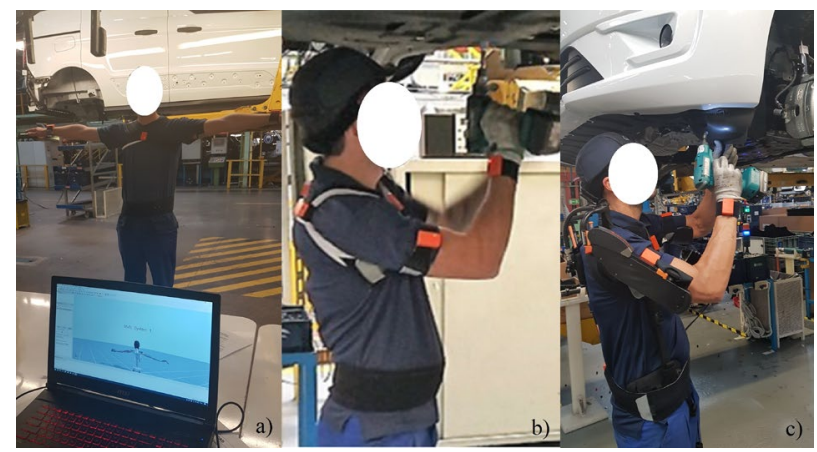

Fig. 3. a) Calibración del sujeto; b) atornillado sin exoesqueleto; c) atornillado con exoesqueleto (Fuente propia)

Seis de los operarios realizaron las actividades sin exoesqueleto, mientras que los otros seis restantes las realizaron con el dispositivo mecánico. El exoesqueleto utilizado para llevar a cabo estas mediciones fue el Levitate AIRFRAME®.

\subsection{Diseño del exoesqueleto en CAD}

Con el objetivo de que las simulaciones sean más visuales, se ha realizado el diseño de un exoesqueleto mediante SolidWorks ${ }^{\circledR}$. Esta representación del dispositivo mecánico está constituida por un apoyo lumbar (1), tres tubos (inferior (2), medio (3) y superior (4)) acoplables, una pieza que se localiza en la parte superior de la espalda (5), dos piezas soporte atornilladas $(6,7)$ dónde se sitúan dos tubos a cada lado del exoesqueleto $(8,9,10,11)$ que actúan como los brazos de éste, un apoyo en la parte posterior del brazo $(16,17)$ y otras dos piezas acoplables $(12,13,14,15)$ que permiten unir este último componente con el tubo superior de cada extremidad. Para simplificar el diseño del dispositivo únicamente se ha realizado el modelado de los componentes rígidos. La Figura 4 muestra el ensamblaje de los componentes en SolidWorks ${ }^{\circledR}$. 

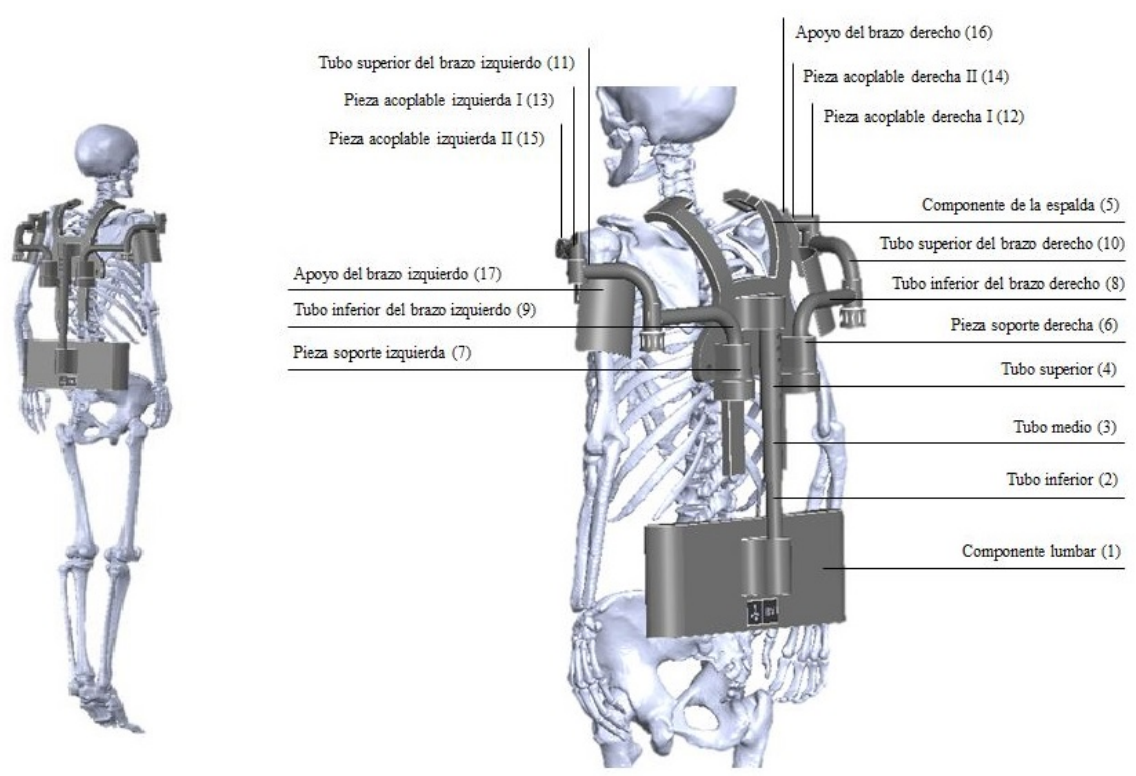

Fig.4. Exoesqueleto diseñado en SolidWorks ${ }^{\circledR}$ (Fuente propia)

Con el objetivo de permitir que el exoesqueleto pueda alcanzar todos los movimientos llevados a cabo por los operarios, se ha dotado a éste de ciertos grados de libertad. La Figura 5 muestra todos los movimientos que pueden ser realizados por el dispositivo mecánico.

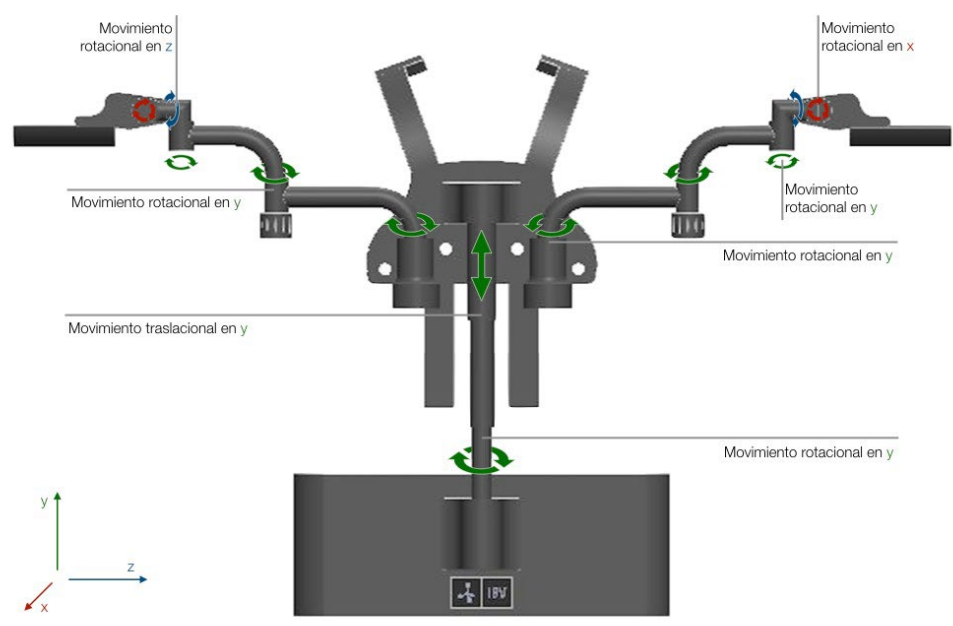

Fig. 5. Grados de libertad del exoesqueleto(Fuente propia) 
A cada uno de estos desplazamientos se le ha proporcionado diferentes rangos de movilidad.

\subsection{Simulación del exoesqueleto}

\subsubsection{Importación de componentes}

Una vez definido el modelo, se han trasladado los componentes de SolidWorks ${ }^{\circledR}$ a OpenSim ${ }^{\circledR}$ - Para ello, primeramente ha sido necesario realizar un cambio del origen del eje de coordenadas de los componentes. Al exportar los archivos de SolidWorks ${ }^{\circledR}$, el origen del eje de coordenadas situado en el componente sufre una variación, de forma que éste queda situado en el primer cuadrante del sistema de coordenadas. Con el objetivo de que durante las simulaciones los componentes puedan rotar y trasladarse a lo largo de los ejes deseados, es necesario situar el origen del eje de coordenadas sobre la parte del componente que se desea que se generen los movimientos.

Los modelos utilizados en OpenSim ${ }^{\circledR}$ son archivos con una extensión .osim, estos archivos pueden ser modificados desde su código. Para introducir nuevos componentes en un modelo ya existente, se debe crear un nuevo cuerpo en el apartado "BodySet". Para definir completamente este cuerpo, se le debe asignar un cuerpo padre ("Parent body"), las coordenadas que tendrá respecto a este componente, su orientación, y los posibles movimientos de rotación y traslación que poseerá la articulación junto con sus rangos de movilidad. La Figura 6 muestra el exoesqueleto importado en OpenSim ${ }^{\circledR}$.

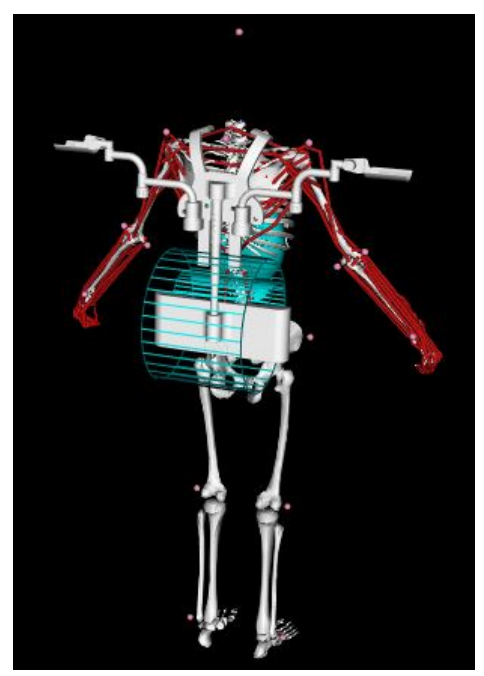

Fig. 6. Modelo en OpenSim ${ }^{\circledR}$ con exoesqueleto(Fuente propia) 


\subsubsection{Escalado del modelo}

El objetivo de escalar el modelo se basa en modificar la antropometría del modelo genérico para que ésta coincida o se asemeje a la antropometría del sujeto registrado. Para ello, OpenSim ${ }^{\circledR}$ ajusta las propiedades de masa y las dimensiones de los segmentos del cuerpo. En este caso, para el dimensionamiento del cuerpo, se ha realizado un escalado basado en la medición.

El modelo utilizado para llevar a cabo este trabajo dispone de 20 marcadores, situados en puntos anatómicos concretos siguiendo los criterios de la ISB (International Society of Biomechanics). Considerando las extremidades superiores como los segmentos de mayor importancia y, sabiendo de antemano que serán los que condicionen el movimiento del exoesqueleto, únicamente se ha realizado el escalado de éstos. El extremo de cada segmento se corresponde con uno de los marcadores empleados y que pueden observarse en la Tabla 1. Solamente se ha llevado a cabo el escalado con los parámetros de aquellos operarios que hicieron uso de la ayuda técnica durante las actividades de atornillado en el registro del movimiento.

Tabla 1. Factores de escalado

\begin{tabular}{cccc}
\hline Segmento & Marcador proximal & Marcador distal & Cuerpo al que se encuentra asociado \\
\hline hombro_d & pIJ (esternón) & pRightAcromion & Clavícula derecha \\
\hline hombro_i & pIJ (esternón) & pLeftAcromion & Clavícula izquierda \\
\hline brazo_d & pRightAcromion & pRightArmLatEpicondyle & Húmero derecho \\
\hline brazo_i & pLeftAcromion & pLeftArmLatEpicondyle & Húmero izquierdo \\
\hline antebrazo_d & pRightMedEpicondyle & pRightUlnarStyloid & Cúbito derecho \\
\hline antebrazo_i & pLeftMedEpicondyle & pLeftUlnarStyloid & Cúbito izquierdo \\
\hline
\end{tabular}

Con el fin de poder realizar una comparativa entre la ayuda mecánica o no hacer uso de ella, por cada modelo escalado, se ha realizado un segundo modelo que dispone de un resorte torsional en cada brazo. Este resorte se ha fijado solamente para el movimiento de "shoulder_elv" (abducción). Las características de este elemento quedan visibles en la siguiente Tabla 2. Los resortes simulan el efecto de ayuda al movimiento que realizaría el exoesqueleto si el operario lo vistiese. 
Tabla 2. Características del resorte

\begin{tabular}{cc}
\hline Rigidez superior (N·m/grados) & $-0.3 \mathrm{~N} \cdot \mathrm{m} /$ grados \\
\hline Límite máximo (grados) & $5^{\circ}$ \\
\hline Rigidez inferior $(\mathbf{N} \cdot \mathbf{m} /$ grados) & $1 \mathrm{~N} \cdot \mathrm{m} /$ grados \\
\hline Límite mínimo (grados) & $-90^{\circ}$ \\
\hline Amortiguamiento & 0.00 \\
\hline Transición (grados) & $2^{\circ}$ \\
\hline
\end{tabular}

\subsubsection{Cinemática inversa}

Una vez realizado el escalado del modelo, se ha procedido a realizar la cinemática inversa. El propósito de la cinemática inversa es hallar los ángulos articulares del modelo que mejor se ajustan a la cinemática experimental de un sujeto en concreto. Para realizar dicha tarea, ésta se basa en las posiciones de marcadores experimentales.

Debido al método de optimización que utiliza OpenSim ${ }^{\circledR}$, la cinemática del modelo produce ciertos errores que impiden que los desplazamientos del exoesqueleto concuerden con los del modelo. Por ello, se han creado dos desplazamientos que simulan las actividades realizadas por los operarios durante el registro del movimiento. Para ello, primeramente se ha generado el movimiento, y posteriormente se ha ajustado el exoesqueleto al modelo cada cierto intervalo de tiempo, registrando los valores que posee cada conexión del dispositivo. Con estos valores ha sido posible realizar una regresión polinomial y obtener unos coeficientes de regresión que han sido aplicados a los valores de los ángulos creados en el archivo de movimiento. El primer desplazamiento creado (ver Figura 7) simula la elevación de la herramienta para realizar el atornillado.

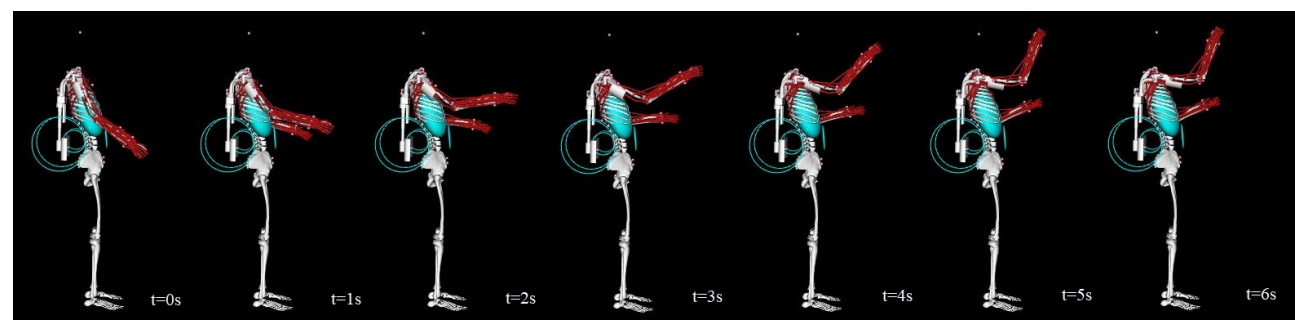

Fig. 7. Movimiento 1 (Fuente propia)

El segundo movimiento (Figura 8) simula cómo el operario desciende la herramienta y eleva el brazo izquierdo para situar el tornillo sobre la herramienta que posteriormente atornillará. 


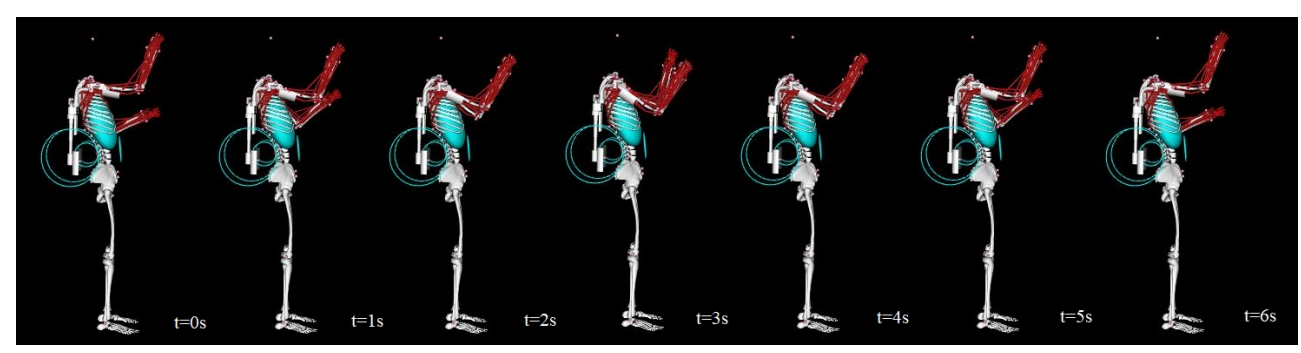

Fig. 8. Movimiento 2 (Fuente propia)

\subsubsection{Dinámica inversa}

El objetivo de la dinámica inversa se basa en estimar las fuerzas y los momentos que causan un movimiento particular. Asimismo, sus resultados pueden ser utilizados para inferir cómo se utilizan los músculos para realizar ese movimiento. En este caso, las fuerzas externas corresponden a un fichero con el valor de la fuerza que simula el peso que posee la herramienta que emplean los operarios durante las actividades en las que se ha registrado su movimiento. Para ello, se ha creado un archivo que presenta una fuerza negativa en el eje " $y$ " (según cómo se ha definido el modelo siguiendo los criterios de la ISB) con un valor de 24,5 $\mathrm{N}$ de peso. Esta fuerza negativa se ha posicionado en un área cercana a la mano con la que el operario sujeta la herramienta. Para ello, se ha tomado la localización del marcador de la mano derecha pRightUlnarStyloid.

\subsubsection{Optimización estática}

La optimización estática utiliza la cinemática conocida del modelo para resolver las ecuaciones de movimiento para las fuerzas generalizadas desconocidas sujetas a condiciones de activación de fuerza muscular. Con el objeto de simplificar el procesado, únicamente se han considerado los músculos del miembro superior derecho, el cual es el encargado de alzar y descender la herramienta de trabajo y, por tanto, el miembro que debe ejercer una mayor fuerza. Asimismo, solamente se han permitido los movimientos de "elv_angle" (aducción), "shoulder_elv" (abducción), "shoulder_rot" (rotación) y "elbow_flexion" (flexión), bloqueando cualquier otro tipo de movimiento que pueda darse. Por último, se ha filtrado la cinemática a $1 \mathrm{~Hz}$ de frecuencia.

A través de la optimización estática se pretende visualizar las activaciones musculares tanto del trapecio superior como del deltoides medio. 


\section{Resultados}

A continuación se presentan los resultados obtenidos para las activaciones del trapecio y del deltoides de tres de los seis operarios analizados. Los resultados se han dividido en los dos movimientos generados. Primeramente, se han obtenido las representaciones de las actividades musculares de ambos músculos con y sin exoesqueleto. Posteriormente, se ha realizado una comparativa de los percentiles p5, p25, p50, p75 y p95 de cada músculo con y sin exoesqueleto.

\subsection{Movimiento 1}

Los valores de los percentiles obtenidos de la activación del trapecio superior para los operarios 1, 3 y 8 , con el uso y sin el uso del exoesqueleto durante el movimiento 1 quedan registrados en la Tabla 3.

Tabla 3. Percentiles obtenidos de las activacines del trapecio durante el movimiento 1

\begin{tabular}{ccccccc}
\hline \multicolumn{7}{c}{ ACTIVACIÓN DEL TRAPECIO SUPERIOR } \\
\hline \multirow{2}{*}{ Operario 1 } & Percentiles & $\mathrm{p} 5$ & $\mathrm{p} 25$ & $\mathrm{p} 50$ & $\mathrm{p} 75$ & $\mathrm{p} 95$ \\
& Sin exoesqueleto & $0.053 \%$ & $0.32 \%$ & $1.48 \%$ & $7.92 \%$ & $48.4 \%$ \\
& Con exoesqueleto & $0.013 \%$ & $0.094 \%$ & $0.91 \%$ & $8.01 \%$ & $29.85 \%$ \\
\hline \multirow{2}{*}{ Operario 3 } & Percentiles & $\mathrm{p} 5$ & $\mathrm{p} 25$ & $\mathrm{p} 50$ & $\mathrm{p} 75$ & $\mathrm{p} 95$ \\
& Sin exoesqueleto & $0.051 \%$ & $0.46 \%$ & $2.66 \%$ & $12.63 \%$ & $44.44 \%$ \\
& Con exoesqueleto & $0.005 \%$ & $0.068 \%$ & $1.04 \%$ & $7.5 \%$ & $31.99 \%$ \\
\hline \multirow{2}{*}{ Operario 8 } & Sin exoesqueleto & $1.05 \%$ & $1.3 \%$ & $2.36 \%$ & $9.28 \%$ & $50.97 \%$ \\
& Con exoesqueleto & $1.01 \%$ & $1.11 \%$ & $1.96 \%$ & $7.57 \%$ & $31.57 \%$ \\
\hline
\end{tabular}

Los valores de los percentiles obtenidos de la activación del deltoides medio para los operarios 1, 3 y 8 con el uso y sin el uso del dispositivo mecánico durante el movimiento 1 quedan definidos en la Tabla 4.

Tabla 4. Percentiles obtenidos de las activaciones del deltoides medio durante el movimiento 1

\begin{tabular}{lcccccr}
\hline \multicolumn{7}{c}{ ACTIVACIÓN DEL DELTOIDES MEDIO } \\
\hline \multirow{2}{*}{ Operario 1 } & Percentiles & $\mathrm{p} 5$ & $\mathrm{p} 25$ & $\mathrm{p} 50$ & $\mathrm{p} 75$ & $\mathrm{p} 95$ \\
& Sin exoesqueleto & $0.006 \%$ & $0.037 \%$ & $0.16 \%$ & $1.3 \%$ & $54.36 \%$
\end{tabular}




\begin{tabular}{ccccccc} 
& Con exoesqueleto & $0.002 \%$ & $0.009 \%$ & $0.12 \%$ & $1.81 \%$ & $44.74 \%$ \\
\hline \multirow{2}{*}{ Operario 3 } & Percentiles & $\mathrm{p} 5$ & $\mathrm{p} 25$ & $\mathrm{p} 50$ & $\mathrm{p} 75$ & $\mathrm{p} 95$ \\
& Sin exoesqueleto & $0.005 \%$ & $0.05 \%$ & $0.31 \%$ & $13.17 \%$ & $71.07 \%$ \\
& Con exoesqueleto & $0.001 \%$ & $0.01 \%$ & $0.14 \%$ & $1.95 \%$ & $52.83 \%$ \\
\hline \multirow{2}{*}{ Operario 8 } & Percentiles & $\mathrm{p} 5$ & $\mathrm{p} 25$ & $\mathrm{p} 50$ & $\mathrm{p} 75$ & $\mathrm{p} 95$ \\
& Sin exoesqueleto & $0.006 \%$ & $0.037 \%$ & $0.16 \%$ & $1.22 \%$ & $45.62 \%$ \\
& Con exoesqueleto & $0.002 \%$ & $0.009 \%$ & $0.08 \%$ & $1.14 \%$ & $40.74 \%$ \\
\hline
\end{tabular}

\subsection{Movimiento 2}

Los valores de los percentiles obtenidos de la activación del trapecio superior para los sujetos 1,3 y 8 , con el uso y sin el uso del exoesqueleto durante el movimiento 2 quedan registrados en la Tabla 5.

Tabla 5. Percentiles obtenidos de las activaciones del trapecio durante el movimiento 2

\begin{tabular}{ccccccc}
\hline \multicolumn{7}{c}{ ACTIVACIÓN DEL TRAPECIO SUPERIOR } \\
\hline \multirow{2}{*}{ Operario 1 } & Percentiles & $\mathrm{p} 5$ & $\mathrm{p} 25$ & $\mathrm{p} 50$ & $\mathrm{p} 75$ & $\mathrm{p} 95$ \\
& Sin exoesqueleto & $1.03 \%$ & $1.15 \%$ & $1.49 \%$ & $3.54 \%$ & $11.08 \%$ \\
& Con exoesqueleto & $1.01 \%$ & $1.05 \%$ & $1.56 \%$ & $2.57 \%$ & $5.90 \%$ \\
\hline \multirow{2}{*}{ Operario 3 } & Percentiles & $\mathrm{p} 5$ & $\mathrm{p} 25$ & $\mathrm{p} 50$ & $\mathrm{p} 75$ & $\mathrm{p} 95$ \\
& Con exoesqueleto & $1.01 \%$ & $1.08 \%$ & $1.27 \%$ & $2.50 \%$ & $7.34 \%$ \\
\hline \multirow{2}{*}{ Operario 8 } & Sin exoesqueleto & $1.02 \%$ & $1.10 \%$ & $1.43 \%$ & $2.48 \%$ & $8.35 \%$ \\
& Con exoesqueleto & $1.01 \%$ & $1.03 \%$ & $1.62 \%$ & $2.61 \%$ & $8.46 \%$ \\
\hline
\end{tabular}

Asimismo, los valores de los percentiles obtenidos de la activación del deltoides medio para los operarios 1,3 y 8 con el uso y sin el uso del exoesqueleto durante la realización del segundo desplazamiento quedan definidos en la Tabla 6.

Tabla 6. Percentiles obtenidos de las activaciones del deltoides durante el movimiento 2

\begin{tabular}{ccccccc}
\hline \multicolumn{7}{c}{ ACTIVACIÓN DEL DELTOIDES MEDIO } \\
\hline \multirow{2}{*}{ Operario 1 } & Percentiles & $\mathrm{p} 5$ & $\mathrm{p} 25$ & $\mathrm{p} 50$ & $\mathrm{p} 75$ & $\mathrm{p} 95$ \\
& Sin exoesqueleto & $0.004 \%$ & $0.076 \%$ & $0.58 \%$ & $3.01 \%$ & $6.95 \%$
\end{tabular}




\begin{tabular}{ccccccc} 
& Con exoesqueleto & $0.001 \%$ & $0.012 \%$ & $0.097 \%$ & $0.18 \%$ & $1.17 \%$ \\
\hline \multirow{2}{*}{ Operario 3 } & Percentiles & $\mathrm{p} 5$ & $\mathrm{p} 25$ & $\mathrm{p} 50$ & $\mathrm{p} 75$ & $\mathrm{p} 95$ \\
& Sin exoesqueleto & $0.0003 \%$ & $0.015 \%$ & $0.041 \%$ & $0.22 \%$ & $1.41 \%$ \\
& Con exoesqueleto & $0.0002 \%$ & $0.005 \%$ & $0.032 \%$ & $0.27 \%$ & $2.28 \%$ \\
\hline \multirow{2}{*}{ Operario 8 } & Percentiles & $\mathrm{p} 5$ & $\mathrm{p} 25$ & $\mathrm{p} 50$ & $\mathrm{p} 75$ & $\mathrm{p} 95$ \\
& Sin exoesqueleto & $0.01 \%$ & $0.081 \%$ & $0.54 \%$ & $1.73 \%$ & $3.86 \%$ \\
& Con exoesqueleto & $0.002 \%$ & $0.011 \%$ & $0.091 \%$ & $0.18 \%$ & $1.65 \%$ \\
\hline
\end{tabular}

\section{Discusión}

Los resultados obtenidos a través de OpenSim ${ }^{\circledR}$ muestran ciertos aspectos a considerar. Como se ha mencionado en el apartado 2.4.2. Cinemática inversa, el primer movimiento generado simula la elevación del brazo derecho del individuo, dónde la extremidad se eleva desde aproximadamente $30^{\circ}$ de abducción hasta $80^{\circ}$. En este movimiento, el trapecio superior trabaja en sinergia con el deltoides medio, provocando el incremento de la abducción en el miembro superior. Sin embargo, aunque la porción acromial (parte media) del deltoides es la más fuerte y es la que comienza el movimiento, las porciones claviculares y espinales participan en grados más altos de abducción. Esto justifica tanto que, a medida que el brazo incrementa su grado de abducción, las activaciones musculares del deltoides medio disminuyan, como que en el segundo movimiento, en el que el brazo ya se encuentra elevado y desciende mínimante para elevarse de nuevo, las activaciones de este músculo sean inferiores respecto al primer movimiento, dado que las mayores activaciones en ese instante en el deltoides se están generando en la parte anterior y posterior. Del mismo modo, al descender el brazo, se atrasa ligeramente el hombro, aproximando la escápula hacia la columna vertebral, función que se encuentra asociada al trapecio medio.

Por otro lado, el resorte torsional situado en el exoesqueleto y que actúa como ayuda durante el movimiento de elevación de los brazos, carece de valor cuando las activaciones musculares son menores. Sin embargo, a medida que las activaciones aumentan, sí que pueden apreciarse disminuciones en las activaciones de ambos músculos cuando se hace uso de la ayuda técnica. Las diferencias que puede suponer hacer uso del exoesqueleto entre un individuo u otro para un movimiento idéntico vienen dadas por los paramétros antropomórficos con los que se ha escalado el modelo.

En la realización del segundo desplazamiento, al operario 3 y 8 les resulta más perjudicial la utilización del dispositivo mecánico que realizar la tarea sin ningún tipo de ayuda. Esto puede deberse al resorte torsional situado en el exoesqueleto. Este elemento ayuda al ascenso del 
brazo, pero sin embargo, puede producir que algunos músculos requieran un esfuerzo adicional cuando se trate de realizar la acción opuesta.

\section{Conclusiones}

OpenSim ${ }^{\circledR}$ puede ser una buena herramienta y primera opción para el análisis de las activaciones musculares que pueden darse en el desempeño de una tarea, ya que puede afirmarse que los resultados obtenidos se ajustan a la realidad. Sin embargo, con el objeto de que estos análisis puedan reflejar una mayor objetividad, sería conveniente no realizar ningún tipo de simplificación y considerar todos los datos antropormórficos posibles de cada sujeto, de este modo, se dispondría un modelo más complejo. Para la obtención de unos mejores resultados en la optimización estática, se debería llevar a cabo una reducción de residuos a través del algoritmo del que dispone OpenSim ${ }^{\circledR}$. Haciendo uso del modelo escalado y del archivo de la cinemática inversa, se pueden realizar ajustes en las propiedades de inercia de los segmentos y de la cinemática conjunta para obtener un modelo y una cinemática dinámicamente consistentes con las fuerzas que posteriormente se aplican.

Asimismo, este archivo de la cinemática resultaría beneficioso obtenerlo a partir de los desplazamientos auténticos de cada uno de los operarios en su ambiente de trabajo.

Analizando los resultados obtenidos, se debería, a su vez, considerar la sustitución del resorte torsional por otro tipo de elemento que simule con mayor fidelidad un actuador pasivo como los utilizados a día de hoy en los exoesqueletos comerciales. De esta forma, el elemento facilitaría la elevación del brazo y permitiría un descenso amortiguado de éste sin la necesidad de realizar ninguna fuerza adicional.

\section{Referencias}

Delp, S. L., Anderson, F. C., Arnold, A. S., Loan, P., Habib, A., John, C. T., ... Thelen, D. G. (2007). OpenSim: Open source to create and analyze dynamic simulations of movement. IEEE Transactions on Bio-Medical Engineering, 54(11), 1940-1950. https://doi.org/10.1109/TBME.2007.901024

Departamento de Información e Investigación del Instituto Nacional de Seguridad e Higiene en el Trabajo. (2012). El TME en el ámbito laboral en cifras.

Kim, S., Nussbaum, M. A., Mokhlespour Esfahani, M. I., Alemi, M. M., Alabdulkarim, S., \& Rashedi, E. (2018). Assessing the influence of a passive, upper extremity exoskeletal vest for tasks requiring arm elevation: Part I - "Expected" effects on discomfort, shoulder muscle activity, and work task performance. Applied Ergonomics, 70(September 2017), 315-322. https://doi.org/10.1016/j.apergo.2018.02.025 
Otten, B. M., Weidner, R., \& Argubi-Wollesen, A. (2018). Evaluation of a Novel Active Exoskeleton for Tasks at or above Head Level. IEEE Robotics and Automation Letters, 3(3), 2408-2415. https://doi.org/10.1109/LRA.2018.2812905

Paulich, M., Schepers, M., Rudigkeit, N., \& Bellusci, G. (n.d.). Xsens MTw Awinda: Miniature Wireless Inertial-Magnetic Motion Tracker for Highly Accurate 3D Kinematic Applications. 1-9.

SolidWorks - Qué es y para qué sirve. (n.d.). Retrieved June 4, 2019, from https://solidbi.es/solidworks/

Spada, S., Ghibaudo, L., Gilotta, S., Gastaldi, L., \& Cavatorta, M. P. (2017). Investigation into the Applicability of a Passive Upper-limb Exoskeleton in Automotive Industry. Procedia Manufacturing, 11(June), 1255-1262. https://doi.org/10.1016/j.promfg.2017.07.252

Sylla, N., Bonnet, V., Colledani, F., \& Fraisse, P. (2014). Ergonomic contribution of ABLE exoskeleton in automotive industry. International Journal of Industrial Ergonomics, 44(4), 475-481. https://doi.org/10.1016/j.ergon.2014.03.008 


\section{TRANSFERENCIA A LA INDUSTRIA Y EL MERCADO}




\title{
Fiabilidad y precisión en Biomecánica
}

\section{Sáenz-Nuño, M. A. ${ }^{a^{*}}$; Pérez-Mallada, N.b; Díez-Sánchez, N.c; Rodeño-Domínguez, M.} ; Moreno-Bellido, F $^{\text {c }}$

${ }^{a}$ Dpto. Ing. Mecánica, Escuela Técnica Superior de Ingeniería, ICAI, Univ. Pontificia Comillas de Madrid,msaenz@comillas.edu,

${ }^{\mathrm{b}}$ Escuela de Enfermería y Fisioterapia San Juan de Dios, Univ. Pontificia Comillas de Madrid, ${ }^{\mathrm{c}}$ Laboratorio de Metrología Dimensional de FREMAP. Servicio de Readaptación Profesional, Hospital de Majadahonda.

\begin{abstract}
There has been developed a lot of new systems for the diagnosis of different illnesses or the measurement of physical parameters. All of them integrated in those small wearables. These equipments allow to have a complete info of the biomechanical variables of interest, without interfering in the diagnosis.

In order to develop realistic and objective models for biomechanical it is is been developed reliable measurements with traceability to the main standards.

In this paper it is presented the successful calibration procedure for a digital goniometer, it has been metrologically characterized, assessing its measurements and estableshing it precision range and accuracy.

This research is the result of a collaboration between an ENAC accredited laboratory and the Universidad Pontificia Comillas, where the system was developed together with other partner.
\end{abstract}

Keywords: Fiability, precision, accuracy, comparability.

\section{Resumen}

Son evidentes las mejoras que se están realizando en los sistemas de diagnóstico utilizados en Biomecánica, con equipos cada vez más integrados en la actividad física a través de diminutos wearables. Ello permite hacer seguimiento de las variables biomecánicas de interés, sin apenas interferir y por consiguiente mejorar la objetividad de los diagnósticos.

Si a esto se aúna la seguridad de unas medidas fiables trazadas a referencias internacionales, se podrán obtener modelos objetivos y realistas biomecánicos totalmente a medida. En el presente trabajo se presenta la experiencia de calibración de un goniómetro digital para su caracterización metrológica, asegurando sus resultados y estableciendo su rango de 
precisión y exactitud. Dicho trabajo se realizó en un laboratorio acreditado por ENAC y con una clara implicación en el sector sanitario. Con ello, todas las mediciones realizadas con este goniómetro van a ofrecer una fiabilidad certificada dentro incluso del sistema industrial, que permitirá garantizar un seguimiento, objetivo y comparable, en la evolución del tratamiento, valoración funcional y pericial de un paciente.

Se presentará también la propuesta de Gestión Metrológica para el equipamiento de medición en Biomecánica, que actualmente se está liderando desde UNE en conjunción con el CEM, Comillas, FREMAP y otras instituciones

Palabras clave: Fiabilidad, precisión, exactitud, comparabilidad

\section{Introducción}

Si bien la medición de ángulos es utilizada desde hace mucho tiempo en los protocolos de análisis de la marcha entre otros, no es muy habitual disponer de trazabilidad en las mediciones. Con el uso de acelerómetros integrados en dispositivos wearables, su uso se ha extendido en la práctica.

En este artículo presentamos la calibración realizada por un laboratorio acreditado en la red ENAC para la completa caracterización del equipo, poniendo de manifiesto la sencillez en la integración del sistema en la cadena de trazabilidad, asegurando así la fiabilidad de sus mediciones.

\section{Goniómetro HAWK para la medición en fisioterapia y el deporte.}

El goniómetro HAWK es un equipo especialmente diseñado para la medición de ángulos en el movimiento del cuerpo humano con una resolución de $1^{\circ}$, siendo un sustituto ideal de los goniómetros tradicionales de división de escala de $5^{\circ}$. Este equipo también se puede utilizar industrialmente para la medición de cualquier ángulo.

Con este estudio se ha caracterizado el funcionamiento del equipo y su respuesta tanto en medición sobre diferentes medios, como el resultado de una medida cuando han transcurrido varios días. De esta forma, se proporciona un ejemplo a seguir para el aseguramiento de las mediciones en otros equipos de uso similar.

\subsection{Descripcion del equipo y su funcionamiento.}

El sensor es un cuerpo de plástico en forma de paralelepípedo que capta el movimiento angular cuando se desplaza en cualquiera de los tres ejes coordenados X, Y y Z (figura 1). 
Si se desplaza el cuerpo de medida en un movimiento angular, éste capta el ángulo mediante un giróscopo interno (según la información facilitada por el fabricante). Esta medición angular se envía por bluetooth a un teléfono móvil que tiene cargada la aplicación del fabricante (APP) para este dispositivo y nos muestra en pantalla los tres ángulos del movimiento realizado, ángulo en relación al eje $\mathrm{X}$, eje $\mathrm{Y}$ y eje $\mathrm{Z}$.
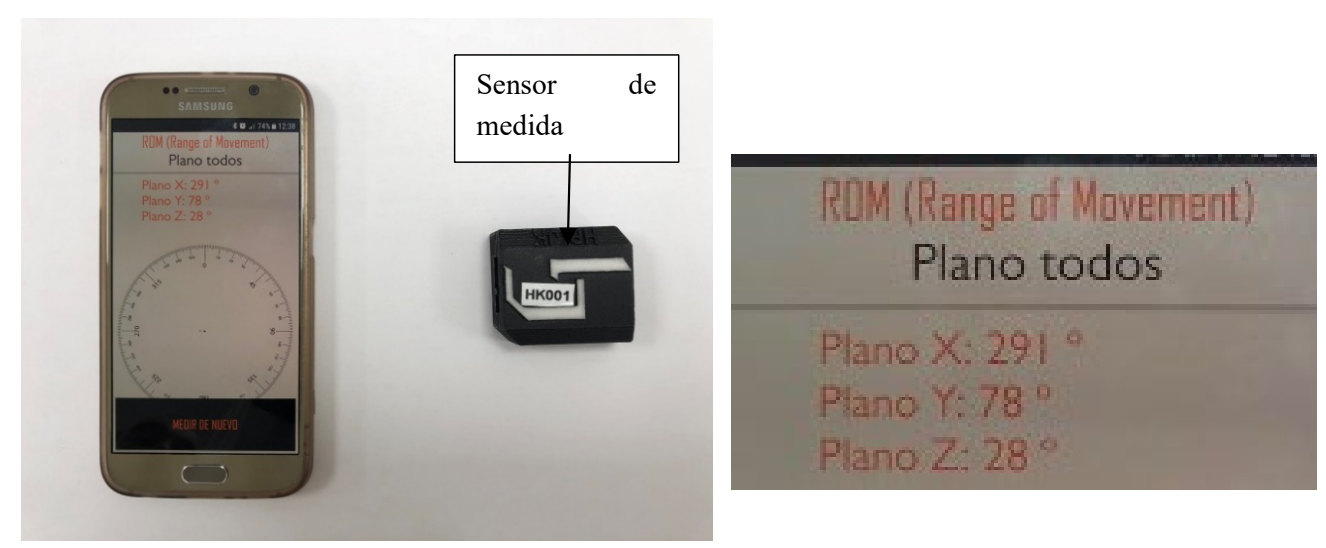

Fig. 1 HAWK. Fuente: Imagen propia (2019).

El equipo tiene un rango de medida de $360^{\circ}$, una división de escala de $1^{\circ}$ y unas dimensiones externas de $43 \times 38 \times 17 \mathrm{~mm}$.

\section{Referencias angulares para la calibración.}

Para realizar el estudio del goniómetro nos planteamos diferentes escenarios de trabajo, de tal manera que pudiéramos ver los errores e incertidumbre de cada uno de ellos y poder concluir con la incertidumbre del equipo en el caso más eficaz, para definir el procedimiento de calibración a realizar. Se hicieron cuatro pruebas:

\subsection{Medida de ángulos sobre plataforma móvil situada en máquina de medida por 3 coordenadas}

Con este ensayo se reprodujo lo más fehacientemente el movimiento del goniómetro sobre una superficie similar a un brazo humano, que es la medida de la plataforma de giro.

La máquina de tres coordenadas trabaja como referencia para la calibración del goniómetro. 

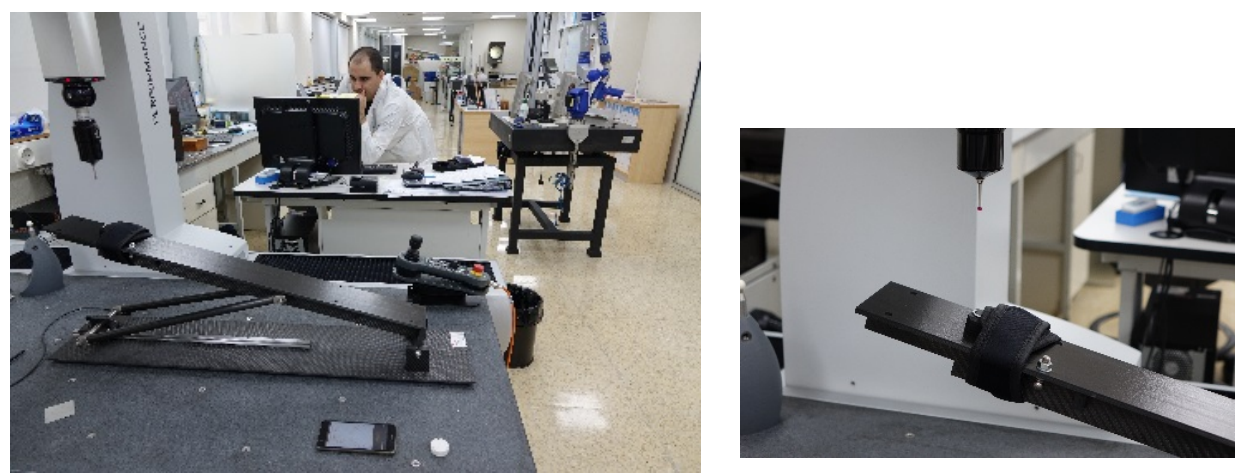

Fig. 2. Medición en la máquina de medición por coordenadas en el laboratorio acreditado de FREMAP. Fuente: Imagen propia (2018).

- Se sujetó el goniómetro sobre la plataforma móvil -- base con giro utilizada para el patrón de bolas-.

- Se midió con la máquina de tres coordenadas calibrada un plano de referencia $0^{\circ}$ sobre la cara del cuerpo del goniómetro, una vez fijado sobre la plataforma móvil, tal y como se aprecia en la figura 2.

- Se colocó la plataforma en posición $10^{\circ}$, según el indicador del mensurando, y se realizó la medición de un plano en la misma zona que el de referencia (se fue abriendo el ángulo de la plataforma hasta que la APP del HAWK indicaba $10^{\circ}$, se fijaba la plataforma y se medía el ángulo con la máquina 3D).

- Se repitió el proceso anterior con los ángulos de $20^{\circ}$ y $30^{\circ}$.

*Nota: las mediciones en la medidora de tres coordenadas se realizaron en modo automático.

\subsection{Medida de ángulos en un solo eje situado sobre proyector de perfiles.}

En este caso, se procedió a la calibración individual de cada eje del HAWK frente a un proyector de perfiles, otro equipo también calibrado y con amplia y variada aplicación en la industria. Con este ensayo se estudió cada uno de los ejes de medición del goniómetro, sin interferencia de medida de los otros ángulos.

Al posicionar el cuerpo del goniómetro sobre la pantalla giratoria del proyector de perfiles, el HAWK medía ángulos en uno solo de los tres ejes X, Y, Z, dependiendo de la superficie del mismo que se apoyase en la pantalla del proyector de perfiles.

Para ello, una vez posicionado el cuerpo del goniómetro sobre la pantalla giratoria del proyector de perfiles, se giraba la pantalla hasta conseguir el ángulo deseado en el HAWK ( $p$ ej. $35^{\circ}$ ) y leyendo el ángulo en la APP del móvil enlazado al HAWK y que actuaba como 
Sáenz-Nuño, M. A.; Pérez-Mallada, N.; Díez-Sánchez, N.; Rodeño-Domínguez,M; Moreno-Bellido, F.

display del mismo. El ángulo patrón era el del giro de la pantalla del proyector de perfiles y con resolución de 1 minuto.

Las pautas que se han seguido para realizar este estudio fueron las siguientes:

- Se eligía la cara principal del HAWK y se colocaba sobre la pantalla giratoria del proyector de perfiles.

- Se escogieron al azar 5 ángulos puros, para ello se giró la pantalla del proyector de perfiles hasta que la APP del HAWK marcaba el ángulo a medir: $10^{\circ}, 45^{\circ}, 65^{\circ}, 72^{\circ}$ y $88^{\circ}$, manteniendo la pantalla inmóvil se tomó un ángulo de giro que fuera el ángulo patrón. Se repitió la operación cuatro veces por ángulo y el juego de medida se repitió tres veces en el transcurso de una hora.
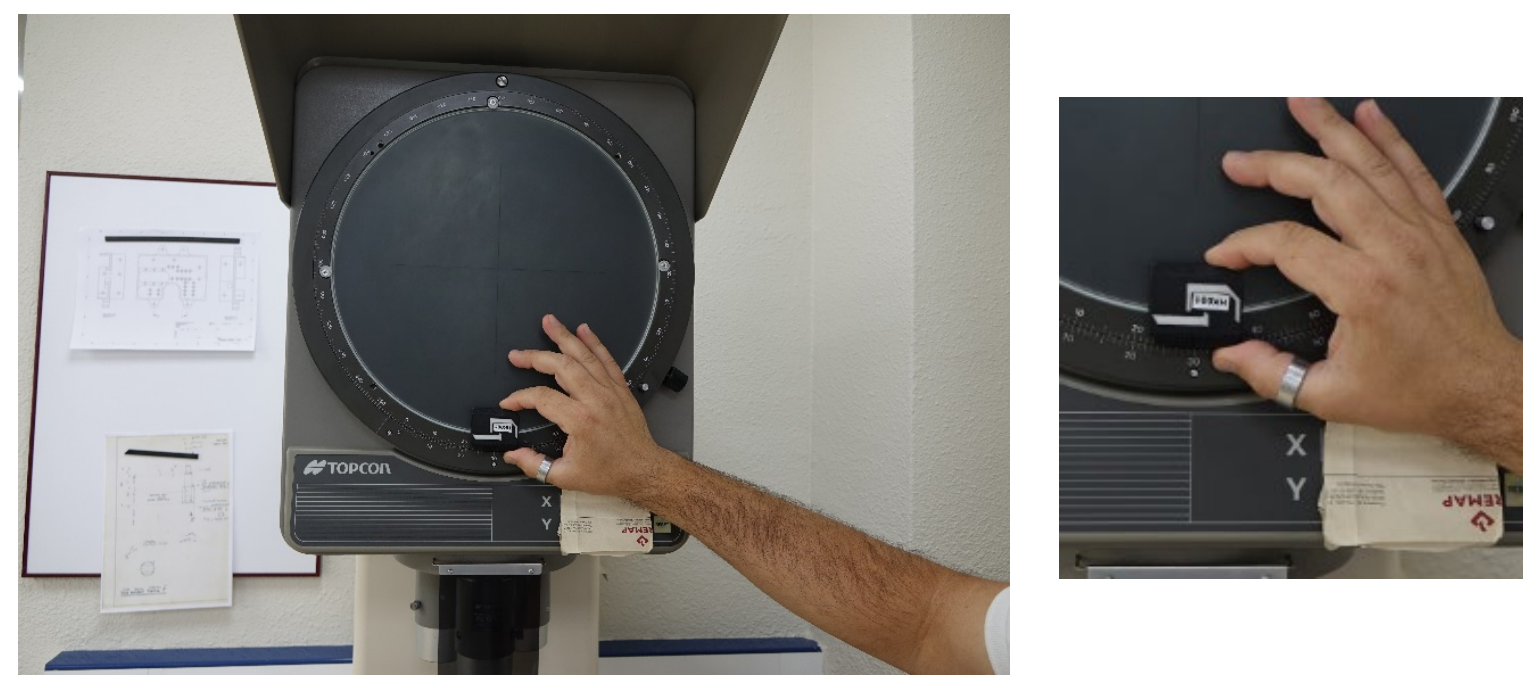

Fig. 3. Medición en el proyector de perfiles en el laboratorio acreditado de FREMAP.

Fuente: Imagen propia (2018).

\subsection{Reproducibilidad del HAWK con proyector de perfiles.}

Se realizó en días diferentes para comprobar si el tiempo entre medida influía en el resultado, asegurando así un valor máximo para la reproducibilidad del equipo.

Siguiendo el esquema de medición de 2.2 se tomaron medidas sobre los ángulos de $72^{\circ} \mathrm{y}$ $185^{\circ}$ en tres días consecutivos.

Se compararon los resultados: medias aritméticas y desviaciones típicas de las 10 medidas repetidas para cada ángulo. 


\subsection{Medida de ángulos sobre proyector de perfiles, posicionando el HAWK en cada una de sus 6 caras y en giro de $90^{\circ}$ en cada una de las caras.}

Se realizó este estudio, con el fin de conocer si la posición del cuerpo del goniómetro afectaba a los resultados de la medición, comprobándose que no era relevante.

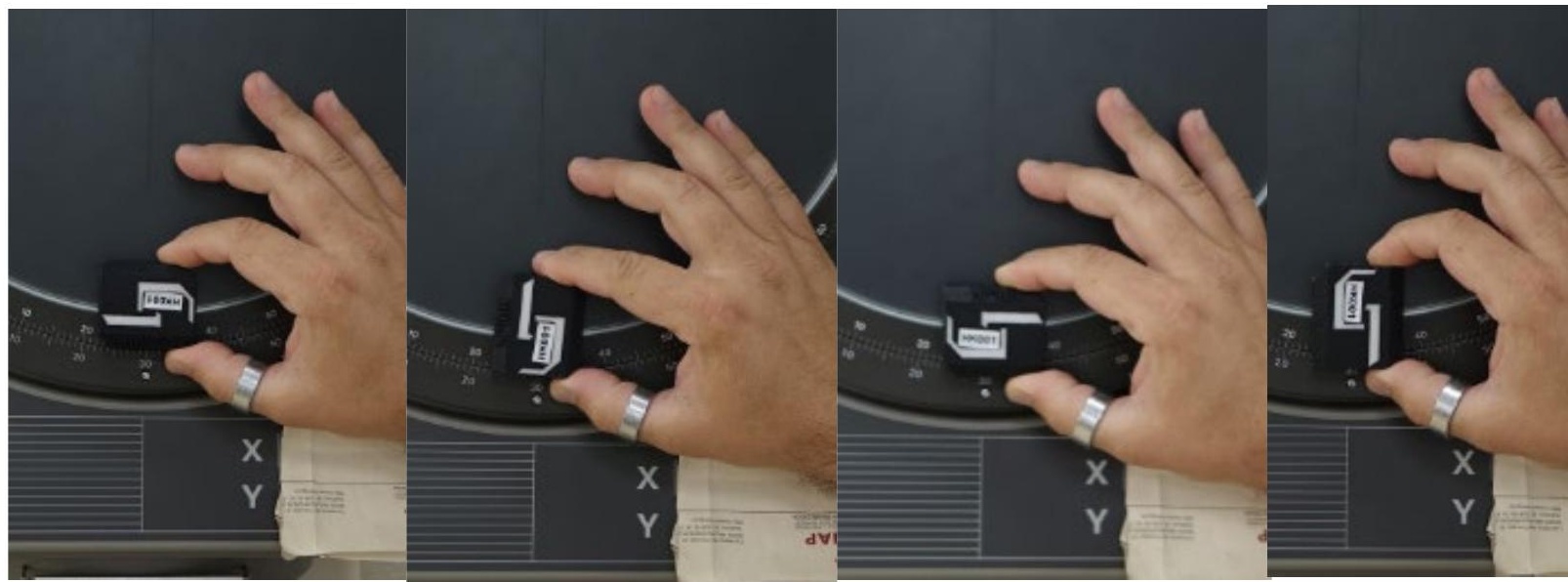

Fig. 4. Medición en el proyector de perfiles en el laboratorio acreditado de FREMAP sobre todas las caras del HAWK (sólo se muestran algunas posiciones).Fuente: Imagen propia (2018).

\section{Adquisición de datos en todos los casos.}

En cada uno de los procedimientos anteriores, la obtención de las lecturas con el equipo fueron:

- Se instaló en un Smartphone el software GONIOMETRO ADVANCE Versión 7.0, disponible en Play Store de Android y que actúa como display del equipo.

- El equipo estaba con la batería cargada al 100\% al inicio de cada serie de medidas.

- Se encendía el goniómetro y se enlazaba mediante bluetooth con la aplicación del Smartphone.

- Una vez encendido y enlazado el equipo, se esperaba cinco minutos para que este se atemperase.

- Se verificaba que el equipo medía en todo su rango, esto es, de $0^{\circ}$ a $360^{\circ}$.

\section{Resultados preliminares.}

Los resultados obtenidos con la máquina de medición por coordenadas en tres ángulos, dieron como resultado una corrección máxima de $0,5^{\circ} \mathrm{y}$ una desviación típica inferior a $0,3^{\circ}$ (véase la tabla 1).

Tabla 1. Medida en grados en la máquina de 3 coordenadas 
Sáenz-Nuño, M. A.; Pérez-Mallada, N.; Díez-Sánchez, N.; Rodeño-Domínguez,M; Moreno-Bellido, F.

\begin{tabular}{|c|c|c|c|c|c|c|c|c|c|}
\hline COTAS & NOMINAL & $\begin{array}{c}\text { MEDIDA } \\
1 \\
\end{array}$ & $\begin{array}{c}\text { MEDIDA } \\
2 \\
\end{array}$ & $\begin{array}{c}\text { MEDIDA } \\
\mathbf{3} \\
\end{array}$ & $\begin{array}{c}\text { MEDIDA } \\
4 \\
\end{array}$ & $\begin{array}{c}\text { MEDIDA } \\
5 \\
\end{array}$ & MEDIA $\square$ & $\begin{array}{c}\text { DES. } \\
\text { TIP }\end{array}$ & $\begin{array}{c}\square \\
\text { CORREC }\end{array}$ \\
\hline 1 & 10 & 9,6108 & 10,1411 & 9,8872 & 9,8691 & 9,5561 & 9,81286 & 0,236212 & $-0,18706$ \\
\hline 2 & 20 & 19,8205 & 19,9588 & 19,848 & 19,9413 & 19,5166 & 19,81704 & 0,178025 & $-0,18286$ \\
\hline 3 & 30 & 29,5255 & 29,5563 & 29,7972 & 29,2877 & 29,3525 & 29,50384 & 0,199312 & $-0,49608$ \\
\hline & & & \multicolumn{3}{|c|}{ MAX DESVIACION TIPICA } & 0,236212 & & & \\
\hline & & & \multicolumn{3}{|c|}{ MAX CORRECCION } & $-0,4960$ & & & \\
\hline
\end{tabular}

Los resultados obtenidos con el proyector de perfiles, dieron como resultado una corrección inferior a $1,5^{\circ}$ y una desviación típica inferior a $1^{\circ}$ (véanse las tablas 2 ).

Tabla 2. Medida de ángulos en un solo eje situado sobre proyector de perfiles en cada eje

\begin{tabular}{|c|c|c|c|c|c|}
\hline \multirow{2}{*}{$\begin{array}{l}\text { Medidas } \\
\text { Eje X }\end{array}$} & \multicolumn{5}{|c|}{ ANGULOS PUROS EN GRADOS : ${ }^{\circ}$} \\
\hline & $10^{\circ}$ & 45 & 65 & 72 & 88 \\
\hline 1 & 10 & 44,766 & 63,816 & 70,416 & 86,783 \\
\hline 2 & 10 & 44,200 & 63,450 & 71,233 & 86,400 \\
\hline 3 & 10 & 44,600 & 63,566 & 70,350 & 86,916 \\
\hline 4 & 10,160 & 43,833 & 63,816 & 70,400 & 86,450 \\
\hline media & 10,040 & 44,350 & 63,662 & 70,600 & 86,637 \\
\hline D. Típica/ ${ }^{\circ}$ & 0,0800 & 0,4185 & 0,1840 & 0,4231 & 0,2519 \\
\hline d. típica/' & 4,800 & 25,108 & 11,041 & 25,386 & 15,111 \\
\hline corrección & $-0,040$ & 0,650 & 1,338 & 1,400 & 1,363 \\
\hline
\end{tabular}

\begin{tabular}{|c|c|c|c|c|c|}
\hline \multirow{2}{*}{$\begin{array}{l}\text { Medidas } \\
\text { Eje Y }\end{array}$} & \multicolumn{5}{|c|}{ ANGULOS PUROS EN GRADOS : ${ }^{\circ}$} \\
\hline & 10 & 45 & 65 & 72 & 88 \\
\hline 1 & 9,933 & 44,416 & 63,500 & 70,666 & 86,966 \\
\hline 2 & 10,200 & 44,583 & 63,616 & 70,616 & 87,583 \\
\hline 3 & 9,900 & 44,566 & 64,250 & 70,250 & 86,450 \\
\hline 4 & 9,916 & 44,416 & 63,583 & 70,583 & 86,333 \\
\hline media & 9,987 & 44,495 & 63,737 & 70,529 & 86,833 \\
\hline D. Típica/ ${ }^{\circ}$ & 0,1425 & 0,0918 & 0,3453 & 0,1889 & 0,5706 \\
\hline d. típica/' & 8,548 & 5,506 & 20,718 & 11,336 & 34,238 \\
\hline Corrección & 0,013 & 0,505 & 1,263 & 1,471 & 1,167 \\
\hline
\end{tabular}




\begin{tabular}{|c|c|c|c|c|c|}
\hline \multirow{2}{*}{$\begin{array}{l}\text { Medidas } \\
\text { Eje Z }\end{array}$} & \multicolumn{5}{|c|}{ ANGULOS PUROS EN GRADOS : ${ }^{\circ}$} \\
\hline & 10 & 45 & 65 & 72 & 88 \\
\hline 1 & 9,900 & 45,116 & 64,516 & 72,000 & 87,316 \\
\hline 2 & 10,083 & 44,900 & 64,666 & 71,566 & 86,850 \\
\hline 3 & 10,083 & 44,716 & 64,683 & 71,683 & 87,616 \\
\hline 4 & 10,083 & 44,650 & 64,500 & 71,866 & 87,150 \\
\hline Media & 10,037 & 44,846 & 64,591 & 71,779 & 87,233 \\
\hline D. Típica/ ${ }^{\circ}$ & 0,0915 & 0,2091 & 0,0966 & 0,1923 & 0,3200 \\
\hline d. típica/' & 5,490 & 12,544 & 5,796 & 11,541 & 19,199 \\
\hline corrección & $-0,037$ & 0,154 & 0,409 & 0,221 & 0,767 \\
\hline
\end{tabular}

Una vez realizado el estudio con el proyector de perfiles, observando los resultados y comparándolos con los del estudio de la máquina de coordenadas, llegamos a la conclusión de seguir realizando el estudio con el proyector de perfiles como patrón ya que con este la incertidumbre que se obtiene es del orden de magnitud de la división de escala, y por tanto, suficiente para las prestaciones del equipo. Suponiendo además un coste significativo en la calibración

Después de haber analizado los resultados obtenidos sobre las medidas realizadas en tres días diferentes consecutivos, llegamos a la conclusión de que el paso del tiempo no influye en el equipo y que por lo tanto esto no influirá en su incertidumbre final. Por lo tanto se consideró que la contribución por reprodubilidad era nula.

\section{Resultados finales.}

La calibración del HAWK condujo así a los siguientes resultados:

Tabla 3. Resultados de la calibración

\begin{tabular}{cc}
\hline Eje & Incertidumbre (k=2) \\
\hline Eje X & $2^{\circ} 18^{\prime}$ \\
Eje Y & $1^{\circ} 4^{\prime}$ \\
Eje Z & $1^{\circ} 1^{\prime}$ \\
\hline
\end{tabular}




\section{Conclusiones.}

Se demuestra la viabilidad de la calibración de instrumentos denominados wearables, con trazabilidad a la cadena industrial, con procedimientos sencillos y fiables.

Se recomienda la utilización de este tipo de procesos para asegurar unas mediciones en el ámbito de la salud, proponiendo la elaboración de nuevos procesos para el entorno sanitario, como es el caso de la Gestión Metrológica Sanitaria.

\section{Referencias}

A. Marín Fernández-Sánchez, A. López-Díaz, C. Jiménez- Alonso, M. Sáenz, J. de Vicente y Oliva. Análisis de las carencias en normalización para la trazabilidad metrológica de equipos médicos. Dyna. vol. 91, no. 5, pp. 536-540, Septiembre 2016. JCR impact factor: 0.522 (2016).

Alfonso Fernández, María Ana Saenz, Nestor Pérez. «Gestión metrológica en la Salud.» AENOR Revista de la normalización y la certifi cación, 2015: 38-43. ISSN: 2255- 0801.

A. Fernández, J.A. Robles Carbonell, P. Centeno de la Torre, G. Ruiz Córdoba, D. Arnal, R. Mur, D. Montes, M. Sáenz, P. Martínez Pallarés. La trazabilidad metrológica en la anestesia. e-medida - Revista Española de Metrología. vol. online, no. 10, Junio 2016 [Online].

Palancar-Martínez, D; Sáenz-Nuño, M. A. (Directora) Trabajo Fin de Master Propuesta de Norma Técnica para la Gestión metrológica en el Sector Sanitario Español, junio 2018. Univ. Pontifi cia Comillas de Madrid.

M. Sáenz, N. Pérez Mallada.’La biomecánica más avanzada se apoya en una metrología de calidad."e-medida - Revista Española de Metrología. vol. online, no. 5, Diciembre 2013 [Online].

UNE 66180:2008 “Sistemas de Gestión de calidad. Guía para la Gestión y la evaluación de los procesos de medición y de confi rmación metrológica”.

UNE EN ISO 10012:2003 “Sistemas de Gestión de las mediciones, requisitos para los procesos de medición y los equipos de medición". 
